\title{
RENEWABLE ENERGY OPPORTUNITES SAGINAW CHIPPEWA INDIAN TRIBE
}

\author{
Final Report \\ DOE Grant: G014252 Saginaw Chippewa Indian Tribe
}

October, 2008

Submitted by:

Planning Department

Saginaw Chippewa Indian Tribe

7070 E. Broadway

Mt. Pleasant, Michigan 48858 
Table of Contents

1.0: The Vision (and its challenges)

1.1: Executive Summary: Renewable Energy and the "New Vision"

2.0: The Problem

3.0: The Tribe Demographic Profile

4.0: The Energy Profile

5.0: Renewable Energy and CHP

5.1: Seventh Generation Renewable Energy Recommendations

5.2: Wind Resource Assessment

5.2.1: Small Wind Power Options

5.2.2: Large Commercial Wind Power Options

5.2.3: Saganing Wind Power Options

5.2.4: Isabella Wind Power Options

5.3: Biomass Combined Heat and Power and District Heat

5.4: Natural Gas Engine Stand-by Combined Heat and Power

5.5: Solar Thermal - Big and Small

5.6: Solar Electric

5.7: Energy Storage, Controls and Integration of Resources

5.8: Energy Efficiency

6.0: Conclusions

7.0: Appendix

A. Wind Conference Brochure

B. Grant Statement of Objectives

C. Small Wind Turbine, and Solar Spec Sheets

D. Small Wood Boiler Specs

E. Saganing Single Turbine Commercial Wind Project Pro Forma's

F. Saganing Wind Farm Pro Forma's and Sensitivity Analysis

G. Isabella Wind Project Pro Forma's and Sensitivity Analysis

H. Biomass Stage I and II Financial Study

I. Gas Engine Cost and details on GE/Jenbacher \& Cummings CHP

J. Phase One Screening Report - Environmental Study

K. Preliminary Cultural Assessment

L. Transmission Study

M. "Power Point" Slide Presentation Slides 


\section{0: The Vision (and its challenges):}

The Saginaw Chippewa Indian Tribe has a vision to become self-sufficient in its energy needs and, in respect and concern for the next seven generations, to maintain its culture and protect Mother Earth.

To achieve this vision, sustainable green energy sources such as solar, wind and biomass energy are the best energy paths to travel. This study provides analysis and details on these and other green technologies illustrating the environmental, economic and energy sovereignty benefits of traveling this path. The results of this study show how this vision can be achieved in less than five years, ali while providing significant economic development benefits to the Tribe.

Most of these green technologies can be immediately justified and implemented at the appropriate scale and with appropriate policies. However, for small individual home applications small windmills, solar electric and some biomass systems can be quite expensive for the renewable energy they produce. Therefore, it is critical to examine and consider the size and scale of each system to optimize the costs and benefits, economically, socially and environmentally. It is also important to consider how each location and each application fits into the total social-economic picture. For example, when considering the capital investment of renewable energy systems one can ask, "how does the Tribe justify delivering potable water to the residence at the far end of the system?" Should the analysis be different for energy? Should clean energy be as important as clean potable water? The Tribe has a top quality, well managed and operated plant and system for water and sewer, there is no reason why it can't do the same for renewable energy systems.

Under narrow economic thinking, where only short-term financial payback is considered comparing new systems to sunk costs (and fossil fuel subsidies, supply chain and other social and environmental costs are ignored), it can be seen that it is difficult to implement renewable energy systems.

We are aware of the challenges of this green path. Converting the conventional fossil fuel infrastructure that reliably provides heat and electricity to the homes, public facilities and commercial facilities to renewable energy is a major change in thinking for operations and maintenance staff. Therefore, the vision and its goals must also include assurances of reliability and economic sustainability. Converting the large Soaring Eagle Casino Resort (SECR) to renewable energy systems may seem especially risky because that facility is the economic engine of the Tribe. The engineering, maintenance and facility operations staff does an excellent job, reliably providing comfort for the visitors that come to this world class resort. Therefore, there must be good justification for retrofitting and/or replacing the energy plants and systems in these facifities. We believe that the information provided below will make the case, and provide 
the justification that the renewable energy systems analyzed below should be implemented by the Tribe as soon as possible.

The Saginaw Chippewa Indian Tribe has a vision to become self-sufficient in its energy needs and to maintain its culture and protect Mother Earth with respect and honor for the next seven generations. To achieve this vision, green energy sources such as solar, wind and biomass energy are the best energy paths to travel.

\section{1: Executive Summary: Renewable Energy and the "New Vision"}

With a total Tribal energy bill for electricity and natural gas of $\$ 5.4$ million in 2007 , over $\$ 75$ million of capital cost investments in renewable can be justified. Put another way, in simple investment terms, if the Tribe could purchase a bond that would return $\$ 5.4$ million a year on the investment, how big of a bond would the Tribe be willing to buy? Renewable energy systems are like purchasing a secure, long-term bond.

With increasing and volatile electric and natural gas energy costs, wind and solar power with zero fuel costs, and biomass (wood chips, wood waste, etc.) with low fuel costs, provide the opportunity to minimize and basically fix energy fuel costs for the Tribe. What is required, however, is the investment in the capital costs for these renewable energy technologies in order to capture and harvest the clean energy that all comes from the sun.

In this feasibility study we have analyzed and provided data on the nature of the renewable resources available to the Tribe and the costs of implementing these technologies. This analysis provides many options. In total these options can provide the needed clean energy not just for the existing facilities, (including public, commercial and residential), but also that for the "new vision" preliminary plans. Table 1 illustrates that these combine energy measures will provide three times the electric needs and three times the thermal (heat and cooling) needs of the existing Tribal facilities. With 2007 annual electric consumption at 51 million kilowatt-hrs/yr and natural gas at 1.9 million CCF $(150,000$ million BTU's per year (MMBTU) adjusted for efficiency), at a capital cost of $\$ 124$ million, this total will roughly meet the energy requirements of the "new vision" plan, including the existing facilities. Table 1 summarizes these costs and energy:

While solar photovoltaic (electric) systems are not listed as a capital expenditure in Table 1 due to its high cost, this technology may have some applications in remote installations, new building structure integration and in emergency stand-by systems. Such solar electric systems have high reliability, low maintenance and long term guaranteed electricity. The Tribe could initiate a solar electric incentive program for its residences to create a large market for a Tribal solar business. Good policies in Europe have caused a boom in solar 
electric installations, and the Tribe could model these successful programs. In the future solar electric systems will play a very large role as costs come down. There are unlimited solar resources for the Tribe to capture, especially during the spring, summer and fall. If cost was not a factor, the Tribe could easily generate its net annual electric consumption of 51 million kW-hrs/year on less than 80 acres of land with existing solar photovoltaic technology.

Table 1: Total Capital Costs, Expenses and Energy

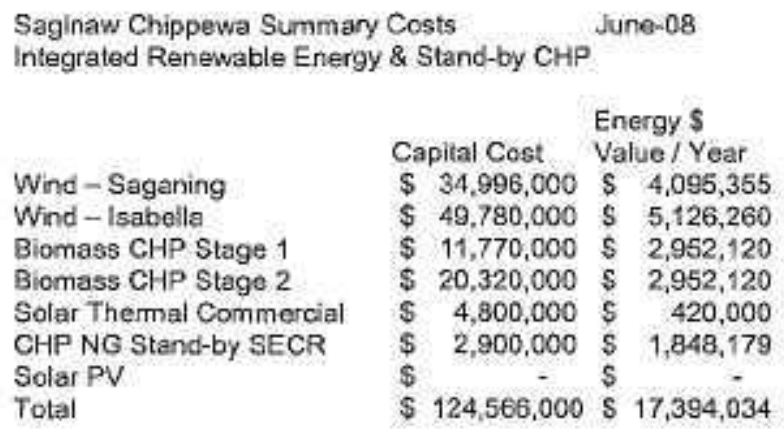

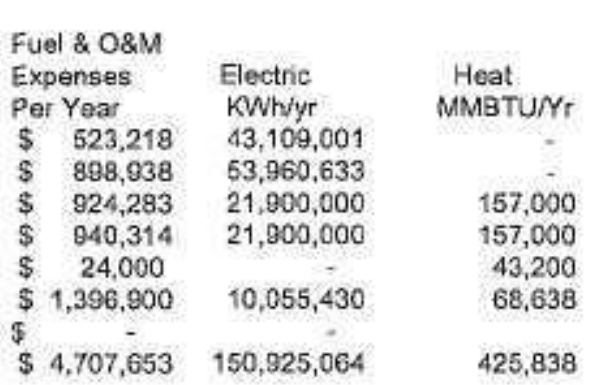

\section{0: The Problem}

One of the biggest problems and challenges for the Tribe is protecting Mother Earth and assuring an economic, cultural and environmental future for the seventh generation. Fossil fuels such as oil and gas and uranium will be gone in one or two generations and the carbon we are pouring into the sky to electrify the Tribe, mostly coming from coal, threaten to dramatically change life on earth in a couple of generations. For these simple reasons, we are in a race against time.

If these fossil resources are not diminished quickly it will be because they will have become too expensive for any to afford, or too prohibitive to burn. Just in the last two years of this study period, fossil fuel costs have become a much greater economic burden. While there is a lot of price volatility, we predict natural gas prices for the Soaring Eagle Casino Resort and residences will be $20 \%$ higher during this coming 2008-09 heating season compared to that of the year before. Delaying one year to install low energy cost renewables such as solar, wind and biomass will have cost an additional $\$ 200,000$ at the SECR and $\$ 300,000$ or more for the Tribe overall with added natural gas energy costs for the 2008-09 heating season. Higher cost expectations exist for electricity as well, with $30 \%$ electric price increases predicted over the next few years. Of course, solar, wind and biomass renewable energy systems have capital costs just as with the fossil fuel systems, but the capital costs paid for renewable energy systems provide energy self-sufficiency. Paying the $\$ 300,000$ per year lost on natural gas for the 2008-09 heating season would finance a lot of renewable energy plant and system capital- $\$ 6$ million at minimum. With a total Tribal 
energy bill of $\$ 5.4$ million in 2007 , over $\$ 75$ million of capital costs in renewable energy investments can be justified. Put another way, the Tribe has a simple choice; does it want to own its energy, or continue to rent its energy?

There are seven major problems ${ }^{1}$ locally, nationally and internationally, associated with the Tribes' use of fossil fuel energy to support its activities:

1. The global climate crisis

2. The exhaustion \& dependence crisis

3. The poverty crisis

4. The nuclear crisis

5. The water crisis

6. The farming crisis

7. The health crisis

${ }^{1}$ See "Energy Autonomy" 2007, Dr. Herman Scheer, pages 36-45.

Global warming is already impacting the indigenous people of the arctic, changing their culture, environment and future. Recent high gasoline costs are increasing poverty levels for those dependent on the automobile, nationally and locally at the Tribe (driving to Saganing, etc.). Over 60 underdeveloped countries have higher fossil fuel import costs than the value of their exports, and therefore they are doomed to spiral further into poverty. Coal and atomic powered electric plants consume more water than any other energy technology. The primary source of pollution in the Great Lakes water comes from the air-when the coal fired power plants deposit mercury, sulfur and other pollutants into air and then from the air to the lakes and land. The negative health effects come both from breathing the polluted air and eating fish with mercury. As a consumer of fossil and atomic fuels the Tribe has responsibility to do its part to address these important problems.

\section{0: The Tribe Demographic Profile}

The Saginaw Chippewa Indian Tribe had the following demographic and geographic characteristics as of 2007:

- Tribe member population $\sim 3,300$

- Isabella 138,240 acres total area (1788 acres in trust)

- Saganing 500 acres (184 ac in trust)

- Residential: $\sim 500$ homes and apartments (trust and non-trust lands) $\sim 600,000$ sq.ft.

- Public Facilities: 24 320,000 sq. ft.

- Commercial: $5+\sim 550,000$ sq. ft. 


\section{0: The Energy Profile}

$\$ 5,412,646$ : This $\$ 5.4$ million is the 2007 annual total energy cost for the Saginaw Chippewa Indian Tribe. This includes commercial, public and residential energy use. Importantly, it does not include vehicle transport, money spent on wood heating systems, higher cost LP gas at residences, and any solar or wind systems Tribal members may be utilizing. Residential energy costs are included, but this cost is based on regional residential energy use averages for natural gas and electricity. Therefore, this cost is a conservative estimate of the Tribes' energy use. With 2008 energy prices increasing significantly it will be safe to assume the total Tribe energy cost, without transport, will exceed $\$ 6$ million per year. While Tribal transportation costs are beyond the scope of this study, we recommend this be included in the next energy studies so there can be a full picture in the Tribal energy use profile. A comprehensive energy use profile, including transportation, should be accomplished as soon as possible.

As shown below in Figure 1, $\$ 3.6$ million or $67 \%$ of the Tribes' energy cost was for electricity and $\$ 1.8$ million or $33 \%$ was for natural gas.

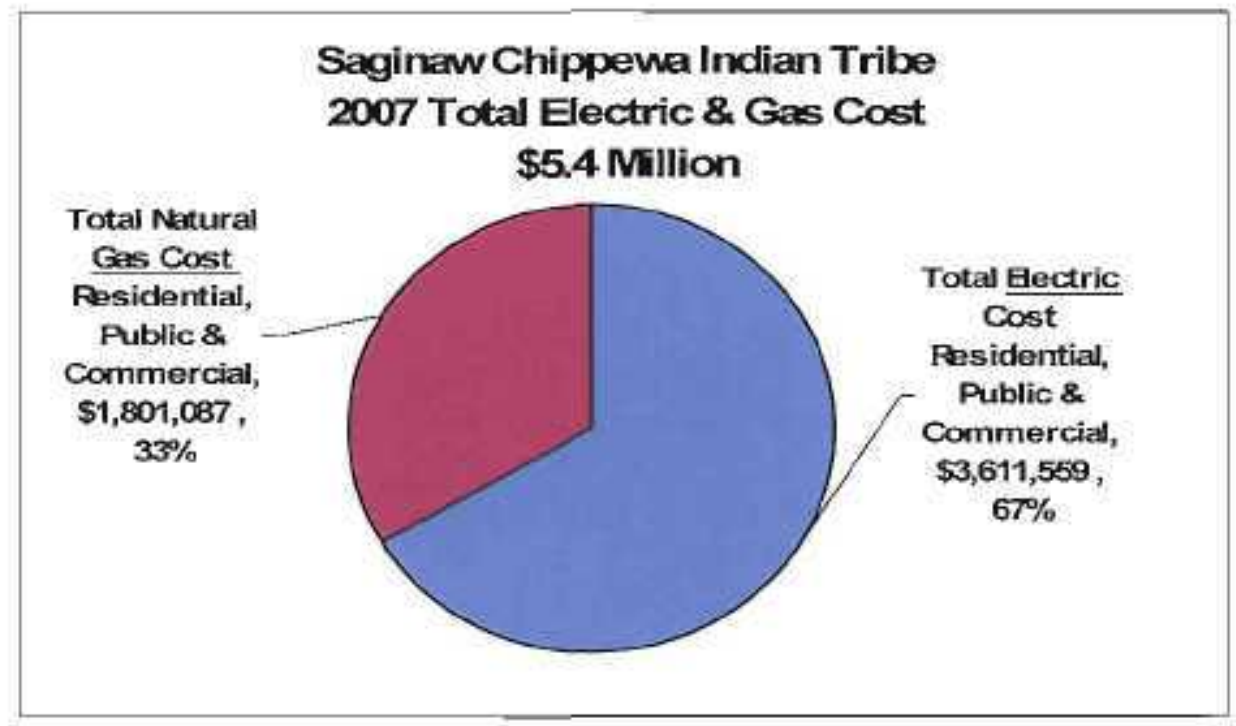

Figure 1

Of the $\$ 3.6$ million dollars per year 2007 spent on electricity purchases, $\$ 2.77$ million, or $77 \%$ went to commercial facilities (see Figure 2), with the large majority for the SECR. $\$ 535,000$ per year, or $15 \%$ of electric costs were for public facilities and approximately $\$ 300,000$, or $8 \%$ was for residential electric consumption by Tribal members during the year 2007. 


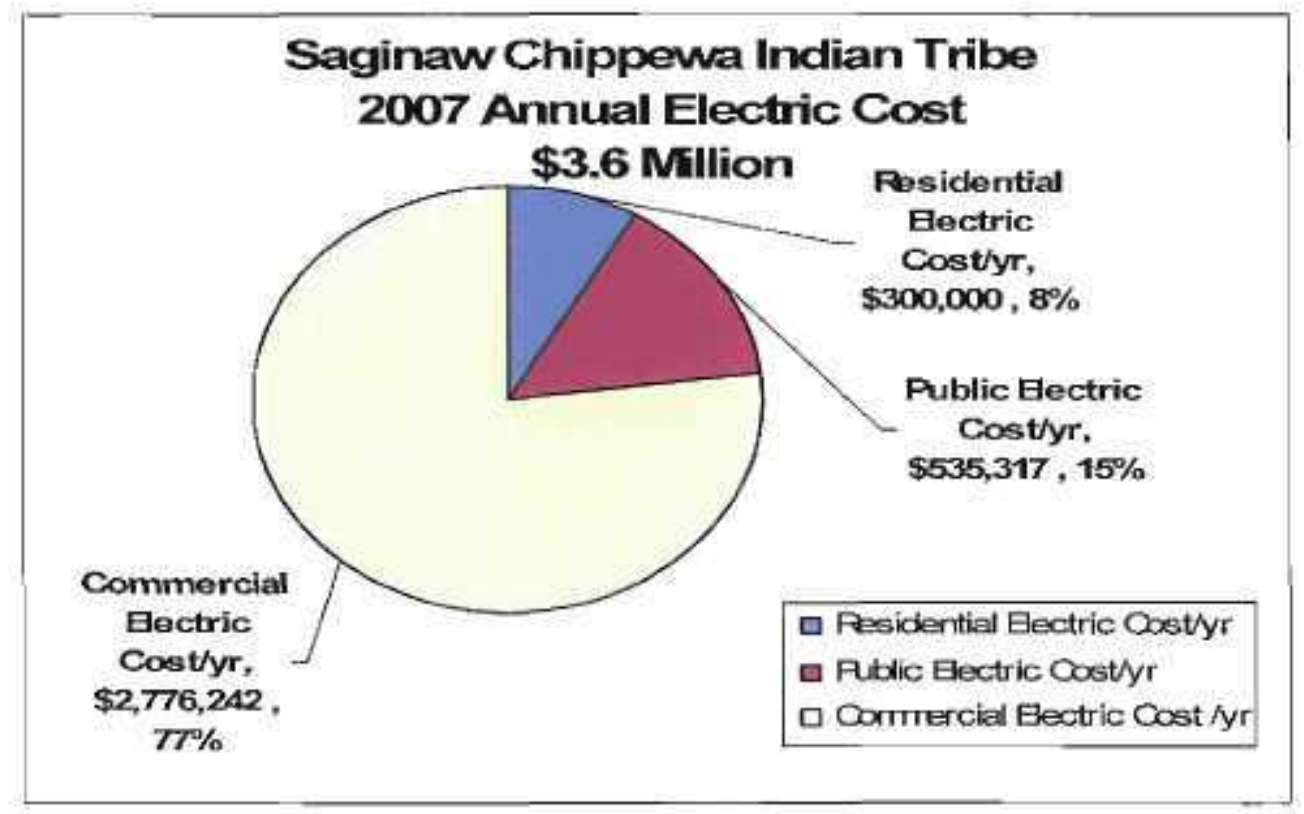

Figure 2

$\$ 1.8$ million dollars per year 2007 was spent on natural gas purchases (see Figure 3 ). Of this amount, $\$ 1.04$ million, or $58 \%$ went to commercial facilities, with the large majority to the SECR. $\$ 350,000$ per year, or $19 \%$ of natural gas purchases were for public facilities and approximately $\$ 407,000$, or $23 \%$ was for residential natural gas consumption by Tribal members during the year 2007.

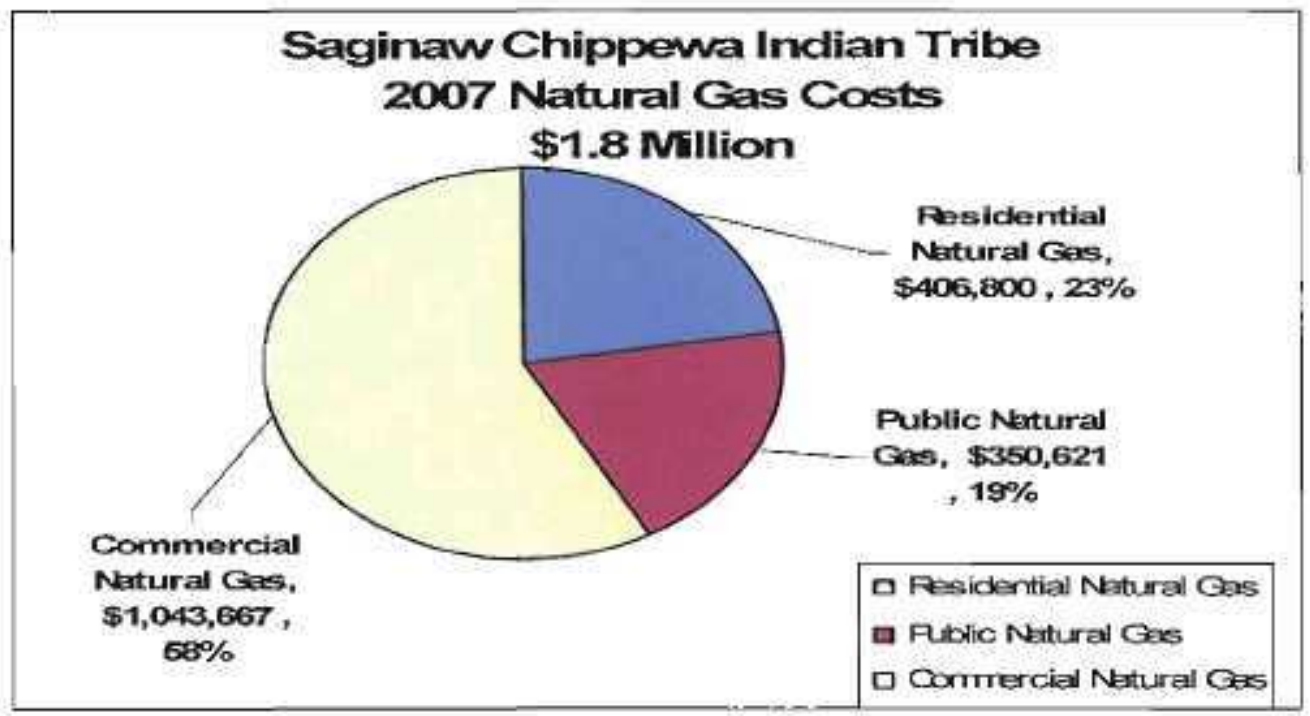

Figure 3 
Total electrical energy consumed by the Tribe in 2007 was 51 million kilowatt-hours (kW-hrs/yr). Most notably, 42 million or $82 \%$ was consumed by commercial facilities, largely from the SECR (see Figure 4).

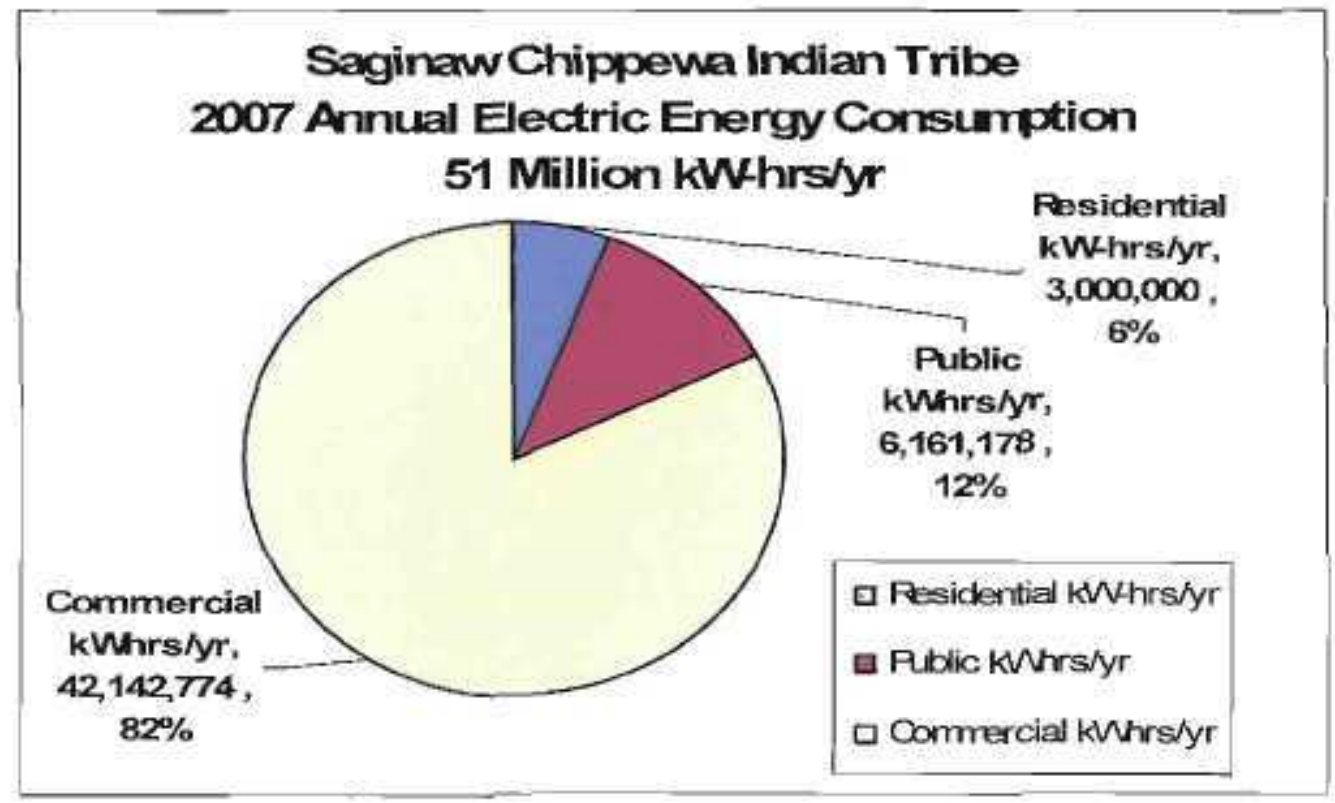

Figure 4

Peak electric demand for the Tribe in year 2007 is approximately 8,833 kilowatts $(\mathrm{kW})$. Peak electric demand is a measure of the highest metered electric consumption during a short period of highest use (usually a 15 or 30 minute average depending on metering)--when the most electrical equipment is operating. Of this peak demand, the SECR accounts for $6,741 \mathrm{~kW}$. The average demand for the SECR is $4,800 \mathrm{~kW}$. This maximum $\mathrm{kW}$ demand of 8,833 can only be approximated due to the lack of meters that record peak demand (see Figure 5). Also, there are "non-simultaneous" peak events, which mean some facilities may not be peaking at the same hour of other facilities, and therefore the overall peak for the entire Tribe is measured to be less than the sum of these estimated peaks. However, statistical electrical "rules of thumb" can be used to make fairly accurate estimates of peak electric demand. 


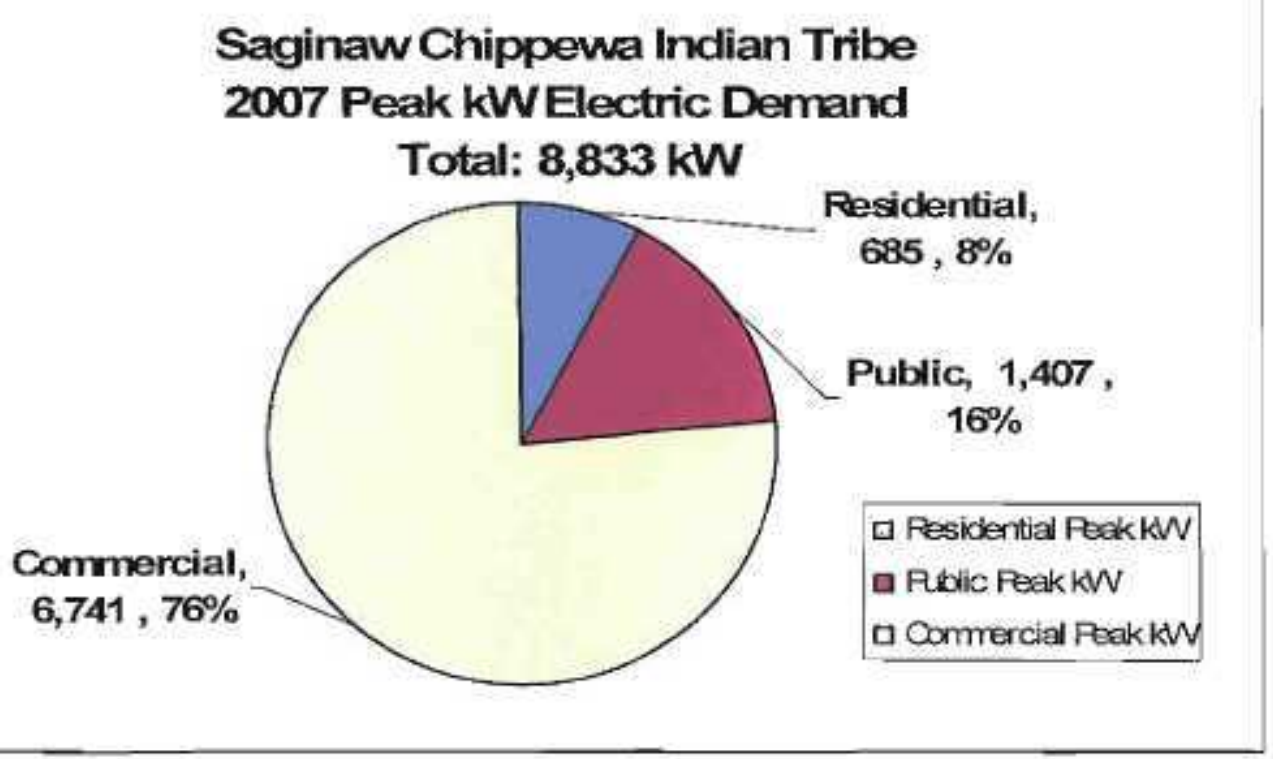

Figure 5

For natural gas, sixty percent $(60 \%)$ or 1.14 million hundred cubic feet (CCF) per year of the total 1.9 million CCF's were consumed by the SECR and related commercial facilities (see Figure 6). While 406,800 CCF's per year, or $21 \%$ of natural gas was used in Tribal member residences in 2007, it is important to note this is the largest part of a residents energy bill, and of most concern to the residences. Also, it should be noted that while LP gas has not been accounted for (due to the difficulty of gathering exact data), its cost is roughly twice that of natural gas for the same heating requirement.

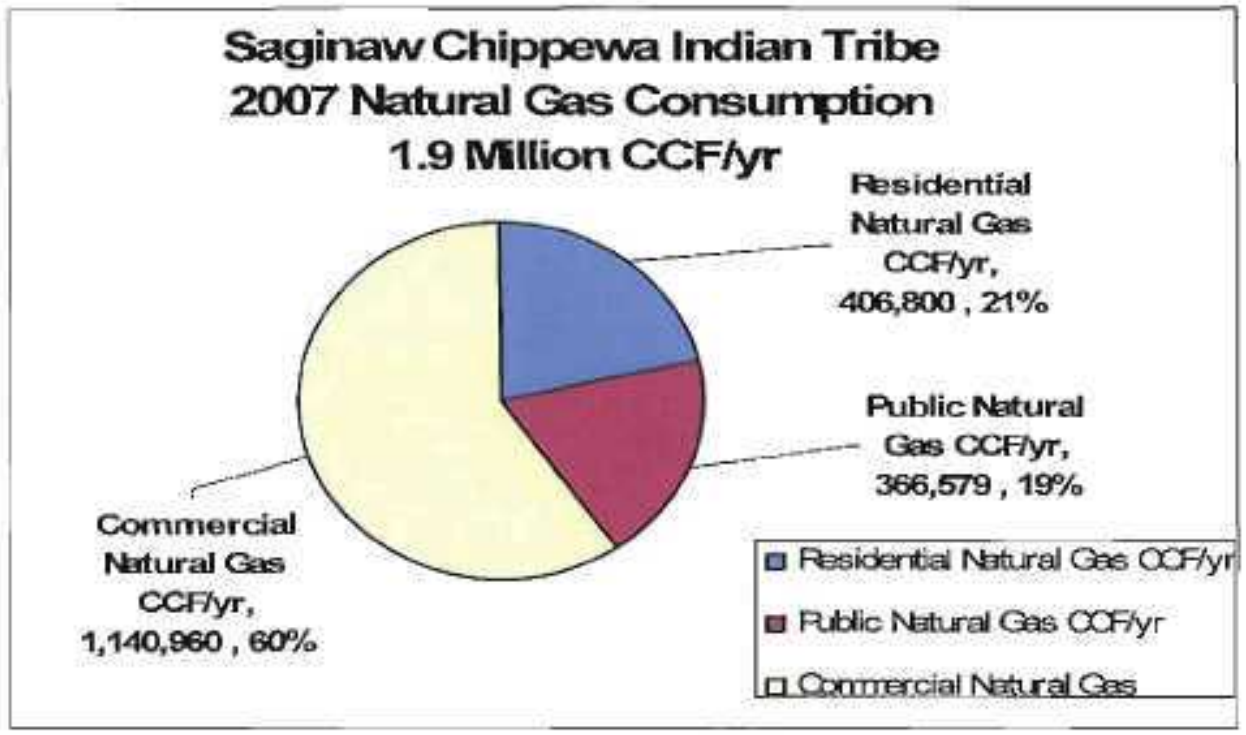

Figure 6 
Establishing this energy profile provides valuable planning information to start on the path to energy self-sufficiency. Important conclusions can be made by looking at the total Tribal energy profile.

To begin, it is shown that electricity use and costs, versus natural gas, are a much greater concern in commercial facilities, especially at the Soaring Eagle Casino Resort, while natural gas and LP gas heating costs are a higher priority for single home residents. Tribal members will individually see home heating "rate shock" this 2008-09 heating season with an estimated 20\% cost increase, while there will not be as much concern by individual Tribal members about energy costs at the commercial facilities since they are not individually impacted. Other conclusions can be surmised from the energy profile, including:

- Individual residential natural and LP gas heating costs are, and will continue to be an important priority for members

- Commercial electric consumption is the biggest part of the budget for the SECR and needs special attention.

- Commercially, natural gas costs, mostly for heating at the SECR, will be the biggest part of the overall energy cost increases in the coming years

- With high peak electric demand at SECR during the hot summer days, added electric capacity from the local grid and substation may soon be required, especially if any expansion plans are initiated. Adding either new generation (electric and thermal heat) and/or significantly expanding energy infrastructure will be required with the "new vision" plans.

- For energy self-sufficiency, new heat and electric generation, based on local renewable energy sources of wind, biomass and solar, should be a priority, in contrast to increasing gas pipe lines, electric lines and substation capacity.

- While it can be shown that providing self-sufficiency for individual residential members is more costly per home due to the distance for district heat piping, higher cost per output small scale wind and solar systems, the benefits are spread over the entire Tribal membership, much like water and sewer. The entire membership is happy when all have access to affordable clean water.

\section{0: Renewable Energy and Combined Heat and Power Technologies}

A comprehensive survey of all practical and proven renewable energy technologies has been undertaken for this study. These include small and large scale wind power, small and large scale solar thermal systems, solar photovoltaics, biomass, district heating with renewables, energy storage and integration of these technologies and resources.

This study originated from a desire by Tribal staff and Councilors to find Seventh Generation solutions, especially with an emphasis on wind power. At 
the beginning of the study focus was put on a wind resource assessment with the installation of two, 50 meter ( $164 \mathrm{ft}$.) meteorological wind test towers and a kickoff event consisting of an all day wind conference for Tribal members and regional community members with interest in wind power (see appendix). The wind conference, attended by over 200 people, was held at the Soaring Eagle Casino Resort and presenters included Winona LaDuke and, Steven B. Smiley.

In addition to the emphasis on wind energy, the objectives of the grant included that; "The Tribe is seeking to become self-sufficient in their energy needs..." And "These efforts are to help the Tribe become sufficient in providing their own energy for the various buildings, cultural centers, schools and residences on Tribe land," and also, "The Tribes' objectives are to provide power for its facilities and potentially create economic development and employment opportunities in its depressed areas..." (See appendix).

Technologies analyzed

- Wind power (big and small)

- Biomass combined heat and power (CHP) and district heat: Stage $1 \& 2$.

- Stand-by natural gas engine electric and heat generation with combined heat and power (CHP),

- Solar Thermal, large scale at the SECR and small scale on each home Solar Electric (photovoltaic)

- Energy Storage and integration: thermal, electric, heat and chilled water

\section{1: Seventh Generation Renewable Energy Recommendations}

Since the initial focus of this study was to examine solutions for energizing the Seventh Generation facilities we begin by review of our results for this facility.

From our studies of Seventh Generation we concluded two basic options should be recommended for consideration. Option one, make the facility partially supplied on-site with renewable energy systems while receiving most of its renewable energy from the larger Tribal renewable energy system, or option two the Tribe can make the Seventh Generation complex completely independent (but not disconnected from the electric grid) in its energy supply with wind, sun and bioenergy.

Option one, involves installing a smaller windmill, a $10 \mathrm{~kW}, 6.7$ meter (22 ft.) wind turbine, 2000 watts of solar electric, a solar thermal system, and interconnection to the Tribal (utility) electric grid and interconnect to a future district hot water heating system. A small biogas digester may or may not be considered as part of this option, however, there may be an interesting opportunity to utilize food waste from the various food services at the Tribe. 
Option two, involves installing a larger windmill, a 100 kilowatt (kW), 21 meter (69 feet) rotor diameter wind turbine, 2000 watts of solar electric, a solar thermal (hot water system), an efficient wood boiler, and a small biogas digester.

For option one, in our analysis, after considering the energy use at the Seventh Generation facilities and the nature of the buildings, and examining many windmill and solar options, we concluded that for electricity, a 10 kilowatt, Ventera VT10 windmill and a 2000 watt solar photovoltaic array (see appendix) will provide an excellent demonstration of wind and solar electric systems, providing roughly $10 \%$ of the annual needs of the electricity for the facilities. We conducted site analysis and wind resource analysis, based on the measured data from the meteorological tower across the road to the north-east and we recommend the proposed the windmill be sited to the north-east of the maintenance garage, east utility pole, 300 feet. This location provides a reasonable distance from the offices and the homes on Remus Road and Shepard Road, with roughly 500 feet to the nearest home and the RTC. It is very unlikely noise will be issue at the RTC however, under very rare circumstances the Seventh Generation staff should be prepared to stop the windmill for short periods of time if it causes disturbances. A photo of the site area is shown in Figure 7.

The cost of the Ventera windmill, with electronics prepared for interfacing the 2000 watts of solar PV, is $\$ 47,000$, installed. This is subject to vary depending on foundation soil conditions. It assumes a 100 foot tower with a 12 foot wind turbine adapter, for a total hub height of 112 feet. The blade rotor diameter is 24 feet.

For option two we propose the wind turbine model NorthWind 100, a $100 \mathrm{~kW}$ peak rated wind turbine with a 21 meter ( 69 feet) rotor diameter on a 37 meter ( 121 feet) steel tube tower (see appendix). This is wind turbine, built in Vermont, is a heavy duty machine, built for higher wind conditions in severe climates and therefore is very reliable, but more expensive. We estimate the installed cost at $\$ 350,000$. Presently, there are not other new wind turbines in this size range that we would recommend. One possibility that we considered, is using used, reconditioned windmills, roughly $20+$ years old, that are available at a lower cost. One example of this is the three $65 \mathrm{~kW}$ windmills installed at the Elkton-Pigeon-Bayport schools in the "thumb" and web link: http://www.energy-now.com/lakerschools/. These three windmills, totaling $195 \mathrm{~kW}$ peak rated capacity ( $65 \mathrm{~kW} \times 3$ ), were built with a $\$ 265,000$ grant. Energy production numbers are available from those wind turbine's, however there is a lot of unknown information regarding operating time and real costs, so without more details it is difficult to make a detailed 
economic comparison. Reconditioned wind turbines like this, however, will be a cheaper installation option at this scale.

There is a web site video on NorthWind 100 in Alaska at http://www.greenenergytv.com/Browse/Wind.aspx?428948141\#428948141

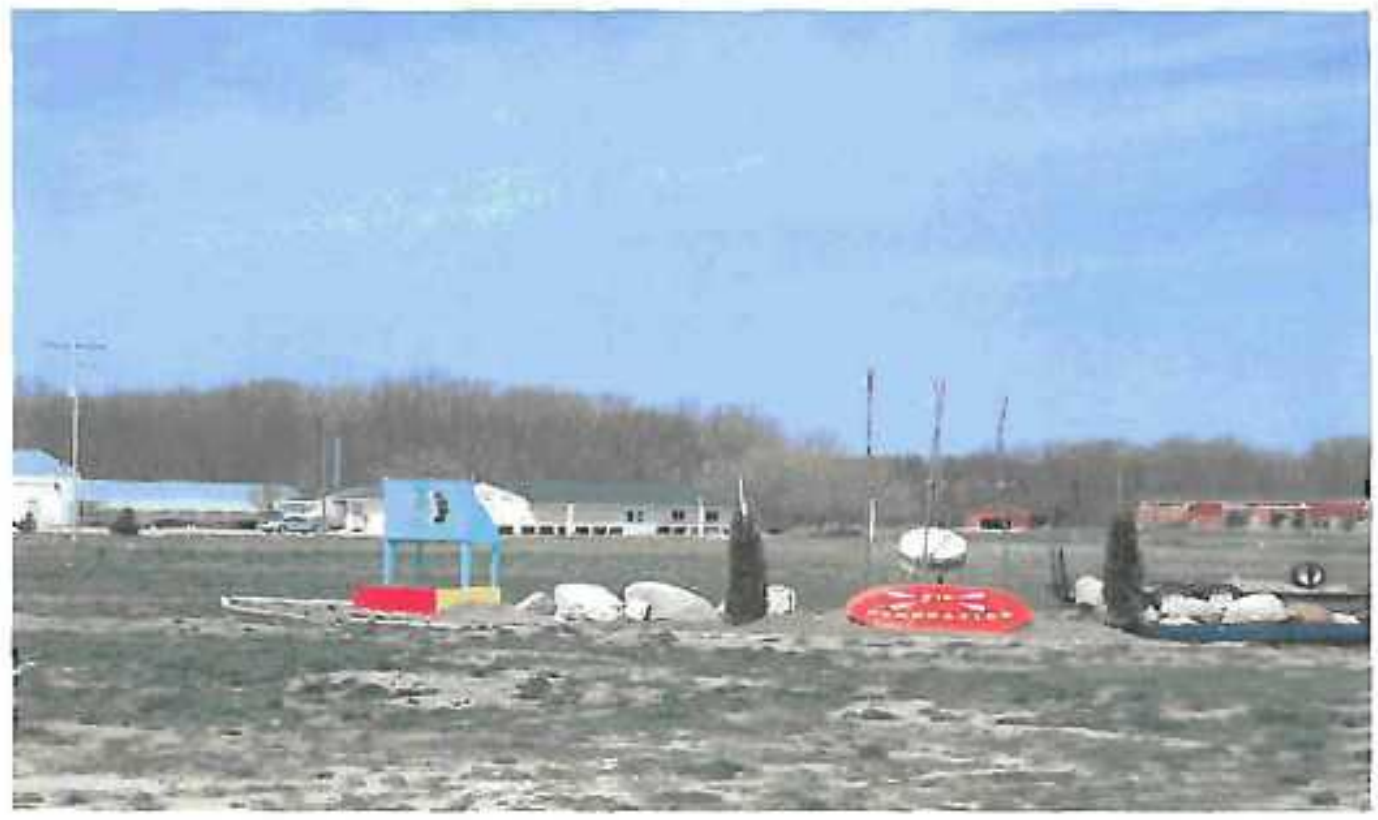

Figure 7

\section{2: Wind Resource Assessment}

A wind resource assessment was completed. In order to conduct this study two fifty meter meteorological towers were installed, one at a site on the Isabella Reservation east of Shepard Road (see photo Figure 8) and one at the Saganing Reservation west of the Eagles Landing Casino on Worth Road (see photo Figure 9). 


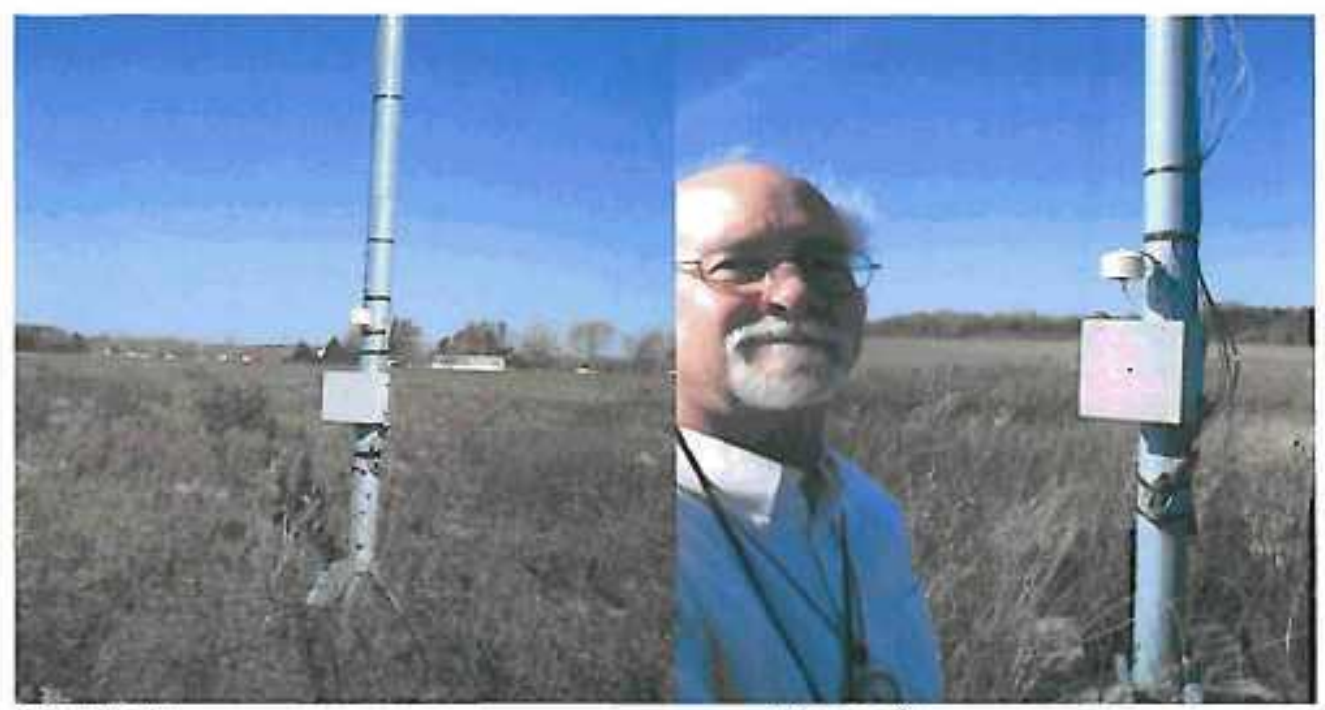

Figure 8

Figure 9

The wind resource study included measurements of wind speed, direction and temperature, with wind speeds at booth towers similarly measured at 30,40 and 50 meters, and direction measured at 40 and 50 meters. From this data wind speed, wind shear factors and wind rose results have been determined and analyzed. While all of the wind sensors on both "met" towers have worked (one, temperature sensor failure), and are continuing to work and gather data, there were some early occurrences of battery failure and lost data. However, since December 2006 , wind data has been successfully collected at a $100 \%$ recovery rate. The following Figure 10 documents sensor operation at the Jsabella site.

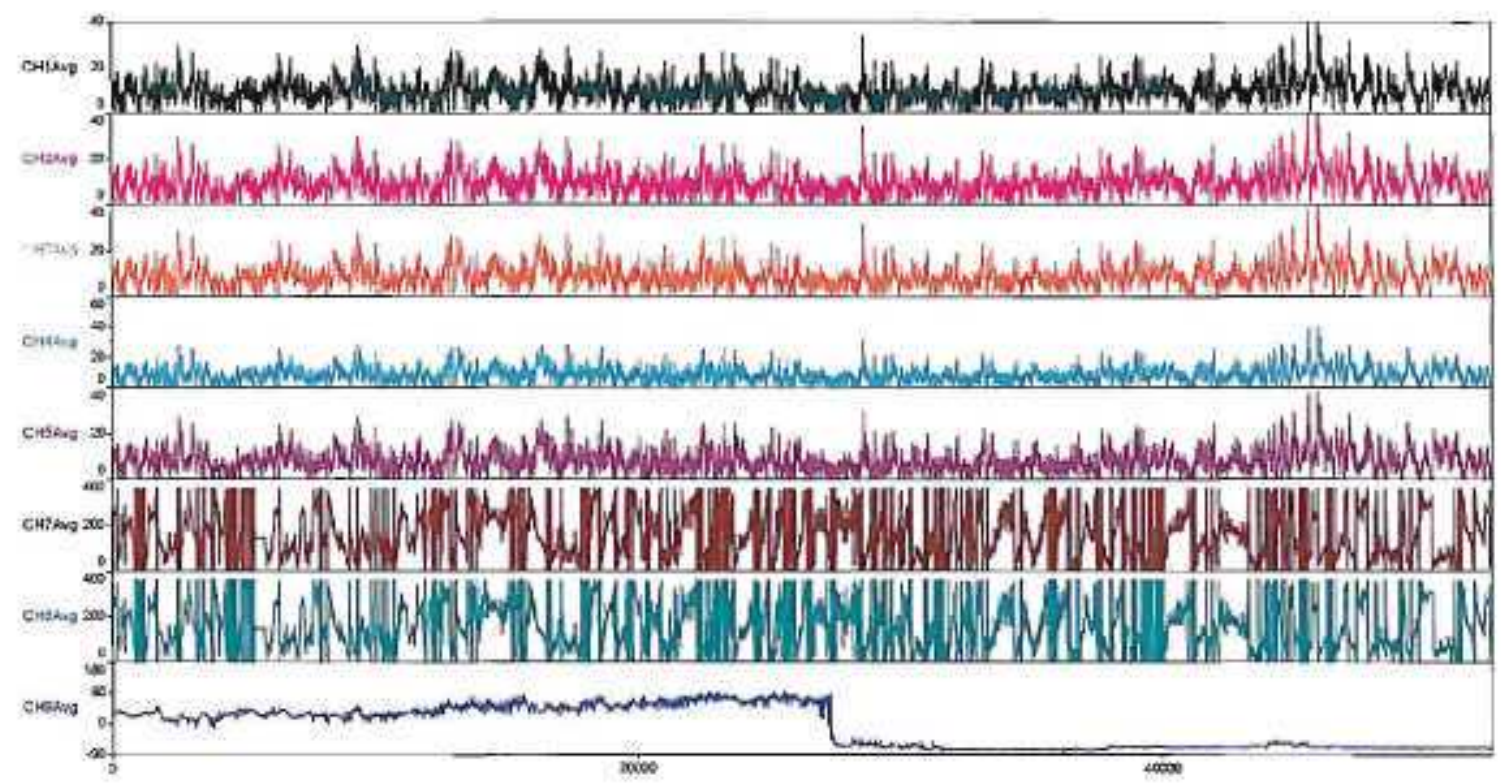

Figure 10 
The general conclusions from the wind resource study are as follows:

- The Saganing site has better wind resources than the Isabella site. (see Figure 11) While both sites have relatively flat terrain within 3 to 5 kilometers $(2-3$ miles), small forested tree stands within the farm lands have the most impact on wind resources and site selection. See the Saganing monthly wind speed graph, Figure 12.

- There is a good correlation of wind speeds between the sites, where Isabella has a coefficient of fit of 0.863 compared to Saganing at the 50 meter level.

- The average annual wind speed at Saganing over the four year period was $10.42 \mathrm{mph}(4.65 \mathrm{~m} / \mathrm{s})$ at the 50 meter $(164 \mathrm{ft})$ height.

- The average annual wind speed at Isabella over the four year period was $9.6 \mathrm{mph}(4.3 \mathrm{~m} / \mathrm{s})$ at the 50 meter $(164 \mathrm{ft})$ height (see Figure 13).

- For the years 2005-2008 there have been historically low winds, and therefore the data collected should provide a conservative long-term projection.

- Wind shear for both sites, from various sensors ranged from an exponent of .17 to .35 with an average of .26 . At a hub height of 80 meters ( $262 \mathrm{ft}$.) we see a $13 \%$ increase in wind speed and at 100 meters (328ft.) we see a $20 \%$ increase in wind speed averages. For Saganing this represents an annual wind speed average increase to $11.8 \mathrm{mph}(5.26 \mathrm{~m} / \mathrm{s})$ at 80 meters to 12.5 $\mathrm{mph}(5.6 \mathrm{~m} / \mathrm{s})$ at 100 meters wind turbine hub height.

- In summary, at a nominal hub height of 80 meters (262 ft.) we can assume an annual average of $5.5 \mathrm{~m} / \mathrm{s}(12.32 \mathrm{mph})$ for Isabella and $6.0 \mathrm{~m} / \mathrm{s}(13.44 \mathrm{mph})$ for Saganing. This will provide a good reference number for comparing wind turbine models. 


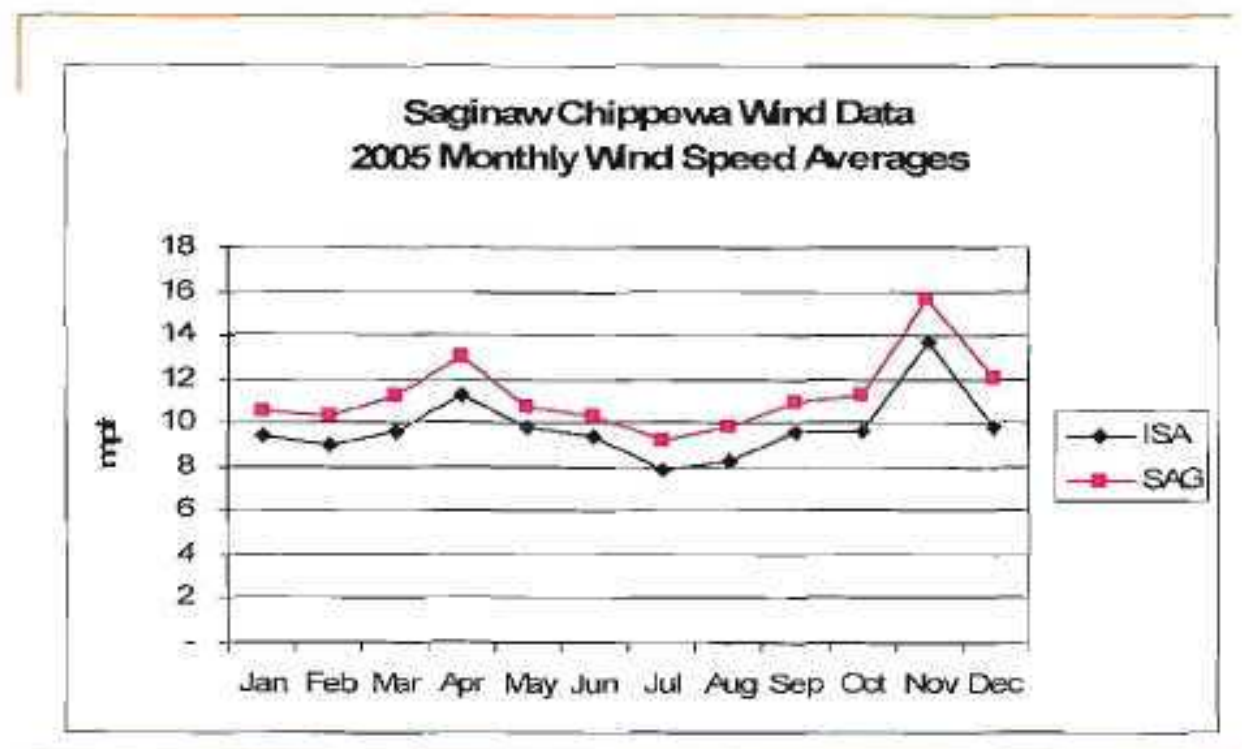

Figure 11

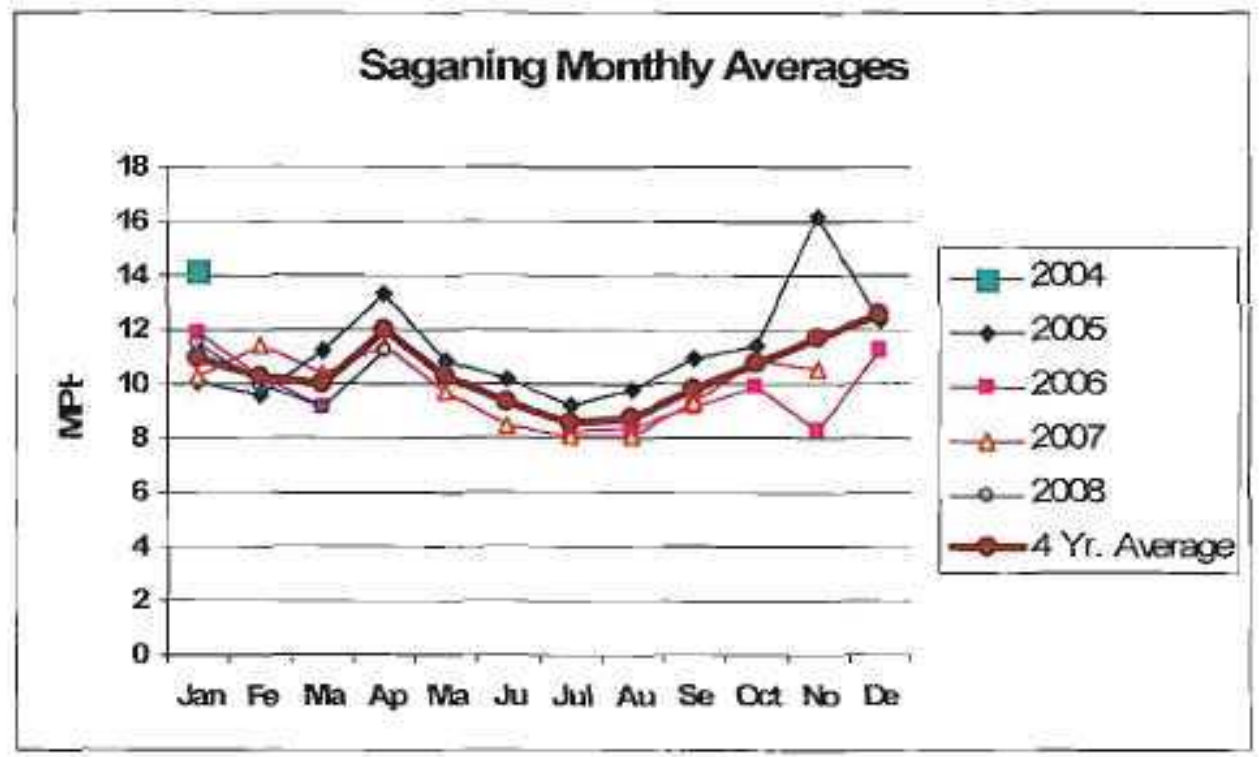

Figure 12 


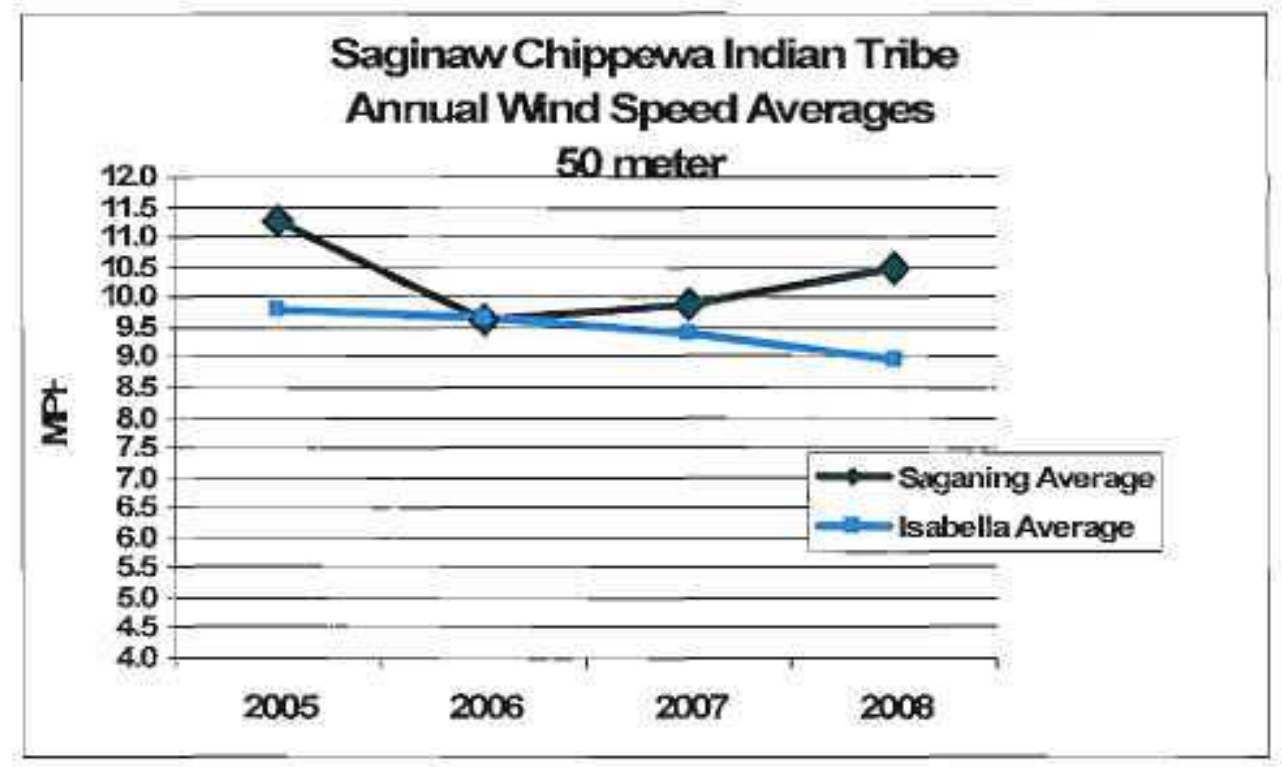

Figure 13

Cost comparisons for small versus large commercial wind energy at the Tribes wind sites illustrate the significant advantages large scale wind energy has over small (less than $100 \mathrm{~kW}$ peak capacity). The following cost curves show per kilowatt-hour (Figure 14) and peak kilowatt installed costs (Figure 15) for both sites.

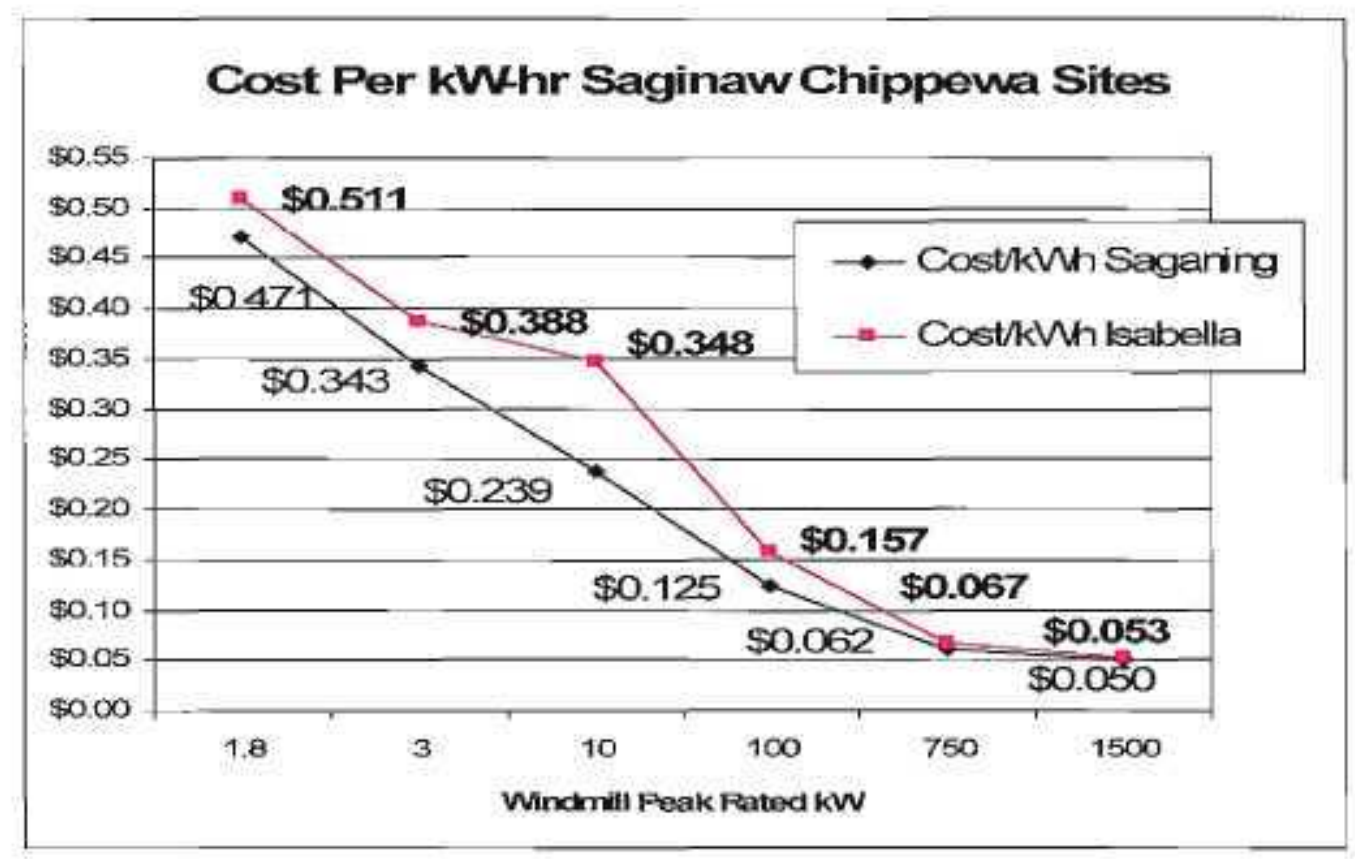

Figure 14 


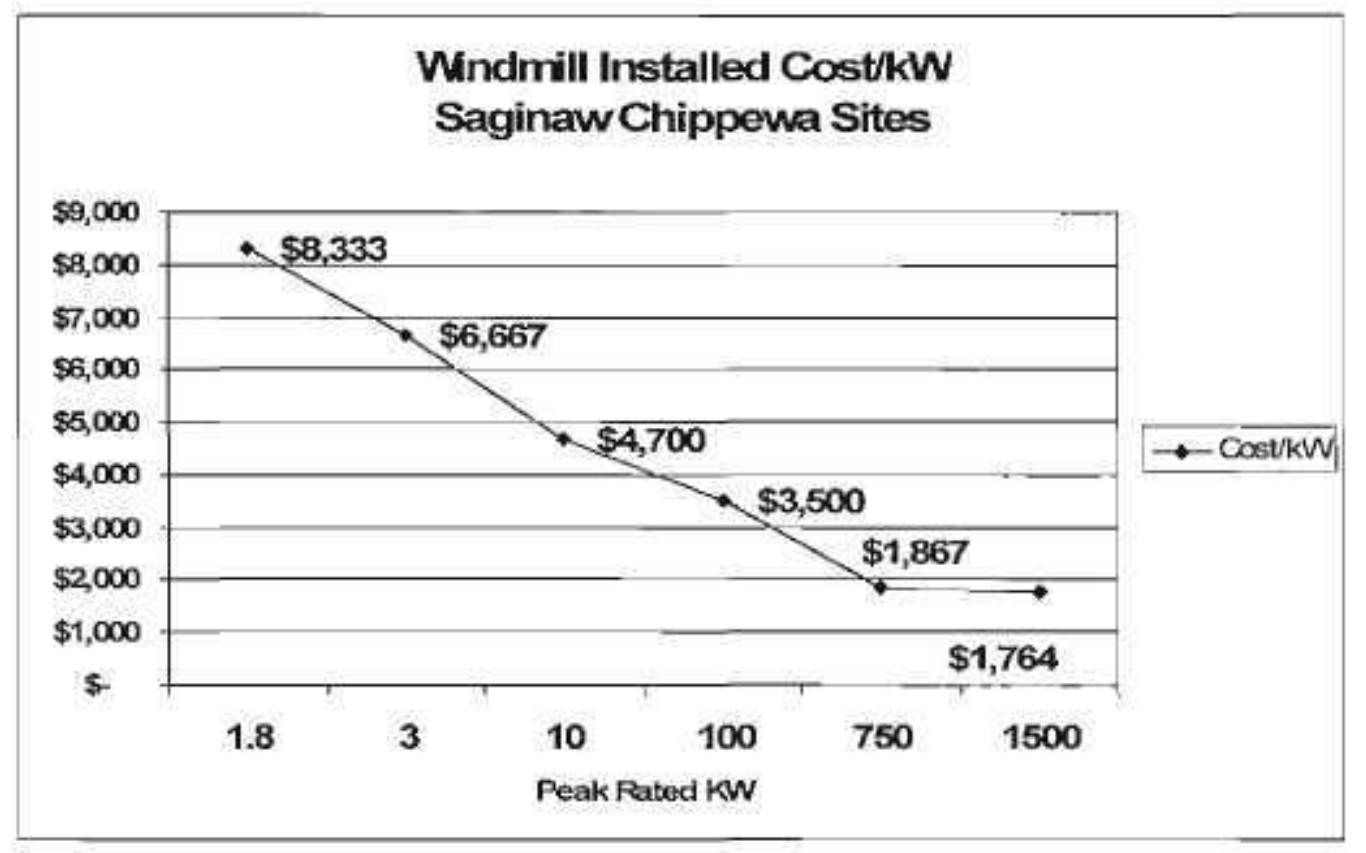

Figure 15

Under present conventional commercial wind industry thinking and project development approaches in the USA these wind resources would not be considered viable. This conventional approach assumes that wind sites are not location constrained, that the electricity payment will come from the lower priced energy only wholesale market. With pollution credits, private tax credits and accelerated depreciation and good wind resources, a large scale project can just be made viable. All of these conventional conditions may be different for the Saginaw Chippewa Indian Tribe.

First, the Tribe can form its own electric utility and make new power generation self-serving, thus increasing the value of the electricity from wind power at its own reservation sites. Next, the renewable energy production incentive payment, presently at 2 cents per kilowatt-hr for ten years, will be available to the Tribe. In addition, new wind turbine models are available that are designed to produce more cost effectively in these lower wind regimes (Euro Class III). Finally, with the expectation of much higher electric prices in the next few years, the Tribe can be offsetting higher retail electric prices, therefore achieving higher savings on the wind power investment. The project economics are very sensitive to future electric price increases, and with a fixed wind energy price, wind power can be cost effective in these lower wind regime areas. Various wind project economic scenarios are provided in the appendix to illustrate the project possibilities at Saganing and Isabella. 


\subsection{1: Small Wind Power Options}

Small electric wind power options available for the Tribe were analyzed, and our general conclusions are that the best opportunities exist where the there are residential or remote applications that are disconnected from the grid, or that the installation will otherwise avoid high grid interconnection costs. Small windmills in the 1 to $10 \mathrm{~kW}$ peak capacity range will provide electricity at costs from 24 cents to 50 cents per kilowatt-hour, depending on the size, site, tower height and other factors. The $100 \mathrm{~kW}$ size range can produce electricity in the 12 to 16 cents per kilowatt-hour range, however the electric generation from this size wind turbine will far exceed a single residential application. This size would be more applicable for a remote commercial or housing district (10 -15 homes).

The models examined included the direct interconnect Skystream 3.4. Whisper 500, Bergey Excel, Ventera and NorthWind 100 (see appendix).

\subsection{2: Large Commercial Wind Power Options}

As shown from the wind power cost curves above large wind turbines in the size range of 750 to $2000 \mathrm{~kW}$ peak capacity can provide the most cost effective electricity. In particular windmill models must be selected that are designed and rated for Euro Class III conditions and optimized for lower wind regime areas. The wind studies indicate that tali towers in the 70 meter $(230 \mathrm{ft}$.) to 100 meter $(328 \mathrm{ft})$ windmill hub height should be considered. The optimal hub height will be dependent on the local terrain and vegetation. All of the Tribe reservation sites are relatively flat ground. Where there are wood lots or small forested areas within a quarter or half mile, a taller tower is recommended. High crane costs and logistics can be an important limiting factor with the larger wind turbines and taller towers. These issues need to be carefully considered when a project is planned for implementation. Recent 2008 installations in eastern lower and mid-Michigan with towers in the 80 and 100 meter range can be examined for comparisons.

With an installed windmill cost of roughly $\$ 1,900$ per peak rated $\mathrm{kW}$, and depending upon financing assumptions, the specific wind site selection, wind turbine selection and hub height, the wholesale cost per kilowatt-hour generated will range from 4.5 cents to 8 cents per $\mathrm{kW}$-hr, accounting for the 2 cent per $\mathrm{kW}$-hr ten year renewable energy production incentive payment available to Tribes. The net present value levelized cost can be below 5 cents per kW-hr, and the benefit/cost ratio will typically fall between 1.5 and 2.0. The return on the investment (IRR 
"Yield") will range from $8 \%$ to $25 \%$, particularly dependent on future conventional electric cost increase projections. In Michigan, high electric cost increases are projected in the next few years, ranging from $10 \%$ to $30 \%$ over the next five years, depending on specific policy and electric utility plans.

Should the Tribe proceed with large commercial wind power it can focus on installing wind power close to the electric consumption at Saganing and Isabella, or consider a remotely based economic development project in better wind resource areas distant from the Tribal lands.

Most commercial wind project developments consist of wind turbines installed in the better wind areas, generating electricity into the transmission system with the electric energy sold at a low price on the wholesale energy market. With additional green credits (REC's or renewable energy credits), power purchase agreements and private tax advantages, projects can be economically viable. Improved payments for electricity sold into the market are guaranteed by a long-term power purchase agreement from a credit worthy buyer, typically an electric utility.

Connecting wind power generation directly into the consumption points and "net metering" is most desirable, avoiding transmission losses and complicated contracts for the delivery of power however, without Tribal ownership and control of the local electric grid, existing utility policy in Michigan limits this opportunity to small wind systems, $30 \mathrm{~kW}$ or less. In addition, commercial wind power must work in conjunction with the electric grid to balance out consumption and generation. When the wind is not blowing, assuming the Tribe has no firm spinning generation such as with a biomass steam turbine, the utility grid power must be available. Either policy changes are needed or the Tribe must directly arrange a special contract with Consumers Energy (the utility service provider) for a significant self-generation system. Consumers Energy is interested in seeing more green power and more electric power in its system. Since the Tribe is major customer of Consumers Energy and the sub-station at Broadway is limited in its capacity, Consumers Energy may be willing to work cooperatively with the Tribe.

Our analysis is focused on the options of making $100 \%$ of the net annual electric consumption of the Tribe at Saganing and Isabella. Presently, Saganing has relatively low electric consumption at residences, the community center and the Eagles Landing Casino, compared to Isabella with the Soaring Eagle Casino Resort (SECR). A single large commercial wind turbine could make all of the electricity consumed at Saganing, while it will take up to 19 large commercial wind turbines at Isabella to make $100 \%$ net annual electric consumption for the Tribe. 


\subsection{3: Saganing Wind Project Options}

Of the two measured wind sites, as shown above, Saganing has the best wind resources. If the Tribe wishes to develop the most economical commercial wind project development on its own lands, Saganing is the best location. Some of the advantages of a Saganing wind project include:

- More available land area owned by the Tribe that is not limited by zoning, buildings, airports or other land-use conflicts or restrictions.

- Access to grid transmission lines that will not limit the size of a project (however a new substation will be needed if the project is larger in scope).

- If a project is developed off Tribal owned lands in the Saganing area under leases from neighboring farms there will be more sites available with an easier zoning and permitting process.

- Better wind resources

A single large commercial wind turbine can make enough net electricity for the Saganing Eagles Landing Casino, other uses, and a potential new small hotel. Generating over 3 million $\mathrm{kW}$-hrs per year, such a wind turbine would be roughly $1,500 \mathrm{~kW}$ or more in peak rated capacity with an 80 meter plus ( $262 \mathrm{ft}$.) rotor diameter. At an installed cost of $\$ 2.9$ for such a wind turbine (without need of a sub-station), the simple payback on such a project would be less than 10 years, a return on investment over $10 \%$, a benefit/cost ratio of 1.4 and a net present value of $\$ 1.7$ million dollars over 25 years. This analysis assumes the inflation rate of conventional electricity is $2 \%$ per year, a conservative estimate. If electric utility purchase rates rise faster than $2 \%$ per year the benefits will be significantly larger. If, for example, an electric inflation rate of $5 \%$ per year is assumed, as predicted by some, the net present value of this project would more than double to $\$ 3.8$ million dollars, for an investment that generates all the clean energy, on a net annual basis, needed by the facilities. The alternative is to continue to pay roughly $\$ 200,000$ per year for electricity, infinitely. This estimated $\$ 200,000$ annual electric bill can be considered the annual budget for the wind turbine. The Tribe may chose: pay for a wind turbine or just pay the electric bill.

"Pro-forma" spreadsheets, with various assumptions, illustrating a range of wind power costs for a single wind turbine at Saganing are provided in the appendix.

Analysis and preliminary design has been completed for a wind project at Saganing that will maximize the use of existing Tribal owned lands in the area. To approximate the total annual electric consumption of the Tribe of 51 million $\mathrm{kW}$-hrs per year, 15 wind turbines is required, 
however a plan with 13 turbines appears to be appropriate for the present available sites, assuming there are no future plans that would be in conflict with these wind sites. The following bullet points summarize the project:

- Total installed capital cost: $\$ 35$ million

- Number of wind turbines: $13 @ 80$ meter + $(262 \mathrm{ft})$ rotor diameter

- Peak rated wind farm capacity: $20 \mathrm{MW}+$

- Cost of electricity: < 5 cents/kW-hr (with REPI)

- Net present value (25 years): $\$ 41$ million

- IRR Yield: $21 \%$

The above financial results assume that the value of electricity off-set or paid is 7 cents per $\mathrm{kW}$-hr (increasing at $2 \%$ per year) and renewable energy credits of 0.5 cents are available. A sensitivity analysis illustrating various financial assumptions is included in the appendix.

\subsection{4: Isabella Wind Project Options}

Of the two measured wind sites, as shown above, Isabelia has the lower wind resources. In order to generate the required 51 million $\mathrm{kW}$-hrs per year to provide $100 \%$ of the net annual electric consumption at the Tribe, approximately 19 wind turbines will be required. Some of the advantages however, of an Isabella wind project include:

- Access to two sub-stations that have lower cost retrofit requirements for interconnection to the Tribe and to grid transmission lines that will not limit the size of a project.

- Close to the large electric loads of the Tribe including the Soaring Eagle Casino Resort, public facilities and residences at the Broadway 10 MVA sub-station, especially if the Tribe establishes its own electric utility.

- The possibility of "net metering" or otherwise having a high value electricity.

- The opportunity to integrate and control the wind power with other electric generation sources, including biomass steam turbines and natural gas $\mathrm{CHP}$.

Siting wind turbines on Tribal owned land is a problem at Isabella due to the height restrictions presented by the Mt. Pleasant airport just north of the Tribal facilities. For this reason the large commercial wind turbines will need to be sited mostly off Tribal owned lands, requiring leases or easements on the larger farms to the south. Twenty-five large commercial wind turbine sites have been identified. Five of these wind turbines can be sited on Tribal lands. The balance would require either Tribal purchase of the lands or long-term wind turbine easements with the land owners. 
"Pro-forma" spreadsheets, with various assumptions, illustrating a range of wind power costs for large project at Isabella are included in the appendix.

Analysis and preliminary design has been completed for a wind project at Isabella that will maximize the use of existing Tribal owned lands in the area. To approximate the total annual electric consumption of the Tribe of 51 million $\mathrm{kW}$-hrs per year, 19 wind turbines are required. A plan with up to 24 sites for wind turbines appears feasible. The following bullet points summarize the project:

- Total installed capital cost: $\$ 49$ million

- Number of wind turbines: $19 @ 80$ meter + $(262 \mathrm{ft})$ rotor diameter

- Peak rated wind farm capacity: $28 \mathrm{MW}+$

- Cost of electricity: 6 cents/kW-hr (with REPI)

- Net present value (25 years): \$39 million

- IRR Yield: $15 \%$

The above financial results assume that the value of electricity off-set is 7 cents per $\mathrm{kW}$-hr (increasing at $4 \%$ per year) and renewable energy credits of 0.5 cents are available. A sensitivity analysis illustrating various financial assumptions is included in the appendix.

\subsection{Biomass Combined Heat and Power and District Heat}

A biomass combined heat and power (CHP) and district heat project for the Isabella Reservation area has been analyzed and a preliminary plan developed. The scope of this analysis focused on providing all of the thermal (heat) requirements for the Tribe at Isabella including the Soaring Eagle Casino Resort, and the other commercial, public and residential facilities.

The sustainable use of biomass, in this case wood chips, provides a cost effective and environmentally preferable energy source. In midMichigan there is a well established market for the harvesting and delivery of wood chips in 30 to 40 ton capacity semi-trailers. The cost ranges from $\$ 20$ to $\$ 26$ dollars per ton, delivered. In addition there are plentiful suppliers of biomass in the form of county and city cuttings from streets, parks and landscaping. Also, the emerald ash bore infestation is requiring the harvest of many ash trees to deal with this serious problem. The neighboring Central Michigan University (CMU) in Mt. Pleasant, Michigan has a $50,000 \mathrm{lb}$ steam wood chip boiler that substantially heats the campus of 15,000 students while also making roughly $900 \mathrm{~kW}$ of electric in a CHP system. The CMU facility contracts for the delivery of green 
wood chips at a price ranging from $\$ 21$ to $\$ 26$ dollars per ton, depending on delivery terms. The County of Isabella handles 35,000 cubic yards of biomass annually, most of which is composted, and handled at a substantial cost to the county. This county biomass supply would provide roughly one-half the heat requirements of the Soaring Eagle Casino Resort at a very low cost of fuel.

At a cost of $\$ 25$ per ton of wood chips, this translates to a price of $\$ 2.79$ per million BTU (MMBTU) compared to a natural gas fuel cost of roughly $\$ 10$ per MMBTU. Natural gas prices have been volatile, and recent "transportation" gas costs to the SECR have ranged from $\$ 9$ to $\$ 13$ per MMBTU including distribution fees. Small residential and commercial Tribal natural gas costs have been around $\$ 10$ per MMBTU, or four times the cost of wood chip biomass fuel. A twenty percent natural gas price increase is expected during the 2008-09 winter heating season. With this low cost sustainable, $\mathrm{CO}_{2}$ neutral fuel supply, a biomass $\mathrm{CHP}$ district heat system is cost effective.

The biomass plant has been sized to meet all of the thermal heating needs of the district and the SECR and large percentage of the electric needs, varying with seasonal operating conditions. A total 60 MMBTU thermal and nominal $5 \mathrm{MW}$ electric steam turbine is recommended.

The biomass CHP district project is planned in two stages. Stage one involves the installation of the biomass CHP power plant at the SECR, close to the largest heat loads. The selected site is to the south of the SECR on the north side of Broadway Avenue, near the employee entrance drive. See the map Figure 16 below. 


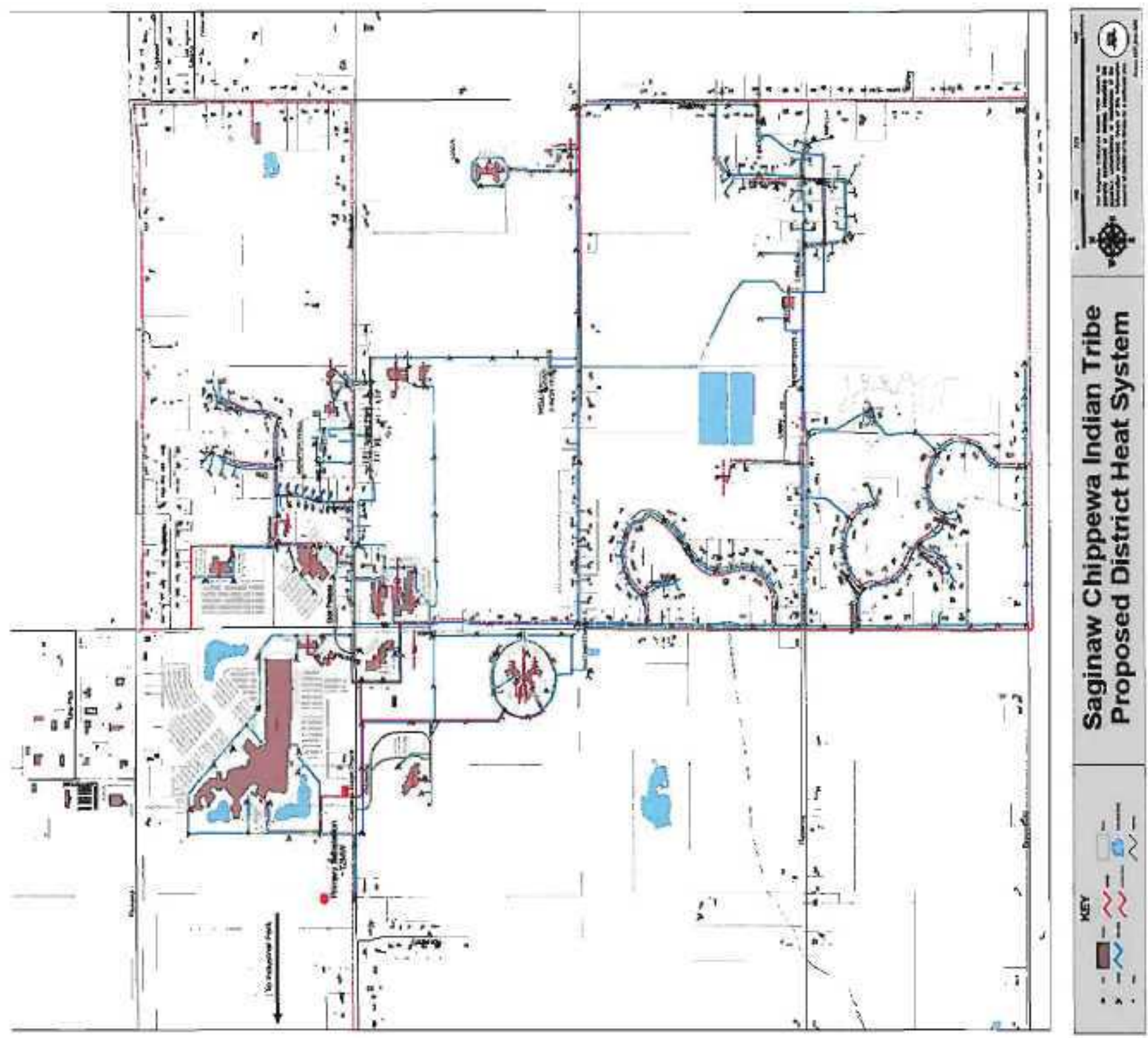

Figure 16

This location for the biomass CHP plant has many advantages. The location is on the mechanical and electrical infrastructure side of the SECR and provides easy access for biomass truck delivery via Broadway Road. Next, the largest heat load, at the SECR is very close to the plant, reducing heat loss and providing short distance heat pipe and electric interconnection, inside the SECR big meter on Broadway. Next, the existing natural gas boiler capacity at the SECR consists of three, 20 MMBTU/hr capacity boilers for a total of $60 \mathrm{MMBTU} / \mathrm{hr}$. (See photo Figure 17) During the summer season much less than $20 \mathrm{MMBTU} / \mathrm{hr}$ heat 
capacity is required, and rarely in the winter are more than two boilers, or $40 \mathrm{MMBTU} / \mathrm{hr}$ heat, required. These existing and relatively new boilers can provide auxiliary or stand-by heat capacity, and the biomass heating system can be directly interconnected to the heating loop of the SECR boilers and the existing controls can be utilized to manage the entire heat distribution system. Finally, there is a concentration of other large commercial and public facilities nearby on and around the intersection of Broadway and Leaton Roads. The interconnection to this concentration of heat and electric loads is the basis for stage one. At an estimated capital cost of $\$ 11.8$ million dollars, it is estimated that heat can be delivered at a cost of $\$ 6.65$ per MMBTU, roughly thirty percent lower than present natural gas costs. The price includes capital, O\&M and fuel costs. Typically there will be required retrofit of the heating systems inside the buildings to accommodate the district heat. This cost will vary from $\$ 1000$ to $\$ 3000$ depending upon the system, and the energy cost savings will justify this investment. Heat delivery will be metered just as with electricity. A breakdown of costs and financial details are included in the appendix.

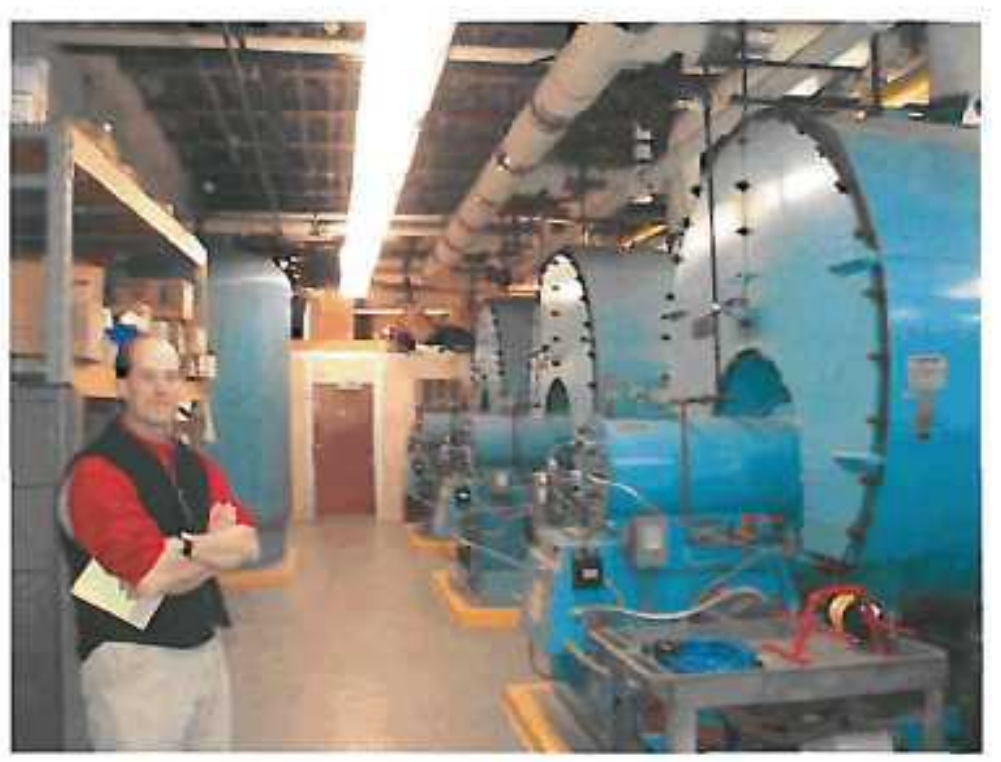

Figure 17

Stage two involves the installation and interconnection of the district heat pipes to all homes, school facilities, and public facilities bordered by Pickard Road (M-20) to the north, Shepherd Road to the east, Broomfield Road to the south and buildings on both sides of Leaton Road. Installing a two pipe heat system to the dispersed Tribal facilities and homes is costly however, this system is fundamentally the same as the existing Tribal district potable water and sewer system operated by the Tribe. In effect, the large economic benefits from stage one off set the higher distribution 
system cost for stage two. At an additional capital cost for stage two of roughiy $\$ 9$ million, and a total system cost of $\$ 20.3$ million the combined cost of delivered heat is estimated to be $\$ 8.99$ per MMBTU, competitive with existing natural gas heating costs.

The cost of electric generation from the CHP biomass plant, utilizing a steam turbine with a nominal $5,000 \mathrm{kWe}$ capacity is roughly 4 cents per $\mathrm{kW}$-hr, with out any federal renewable energy production incentive (REPI) payments. These incentive payments may be available for ten years, however, the US congress must fund the program adequately to guarantee the money, and there are no assurances the money will be available.

If the $5,000 \mathrm{kWe}$ steam turbine operated at a $50 \%$ capacity factor the electric generation would provide roughly 22 million $\mathrm{kW}$-hrs per year, or $40 \%$ of the total Tribal electric consumption. The steam turbine could run at a much higher capacity level, nearly doubling the output however, the optimal electric generation must be determined by an evaluation of the heat requirements for the total district, balanced with the electric requirements. While the peak electric demand is roughly $8,800 \mathrm{kWe}$ and the SECR around $6,700 \mathrm{kWe}$, the average $\mathrm{kWe}$ demand at the SECR is roughly $4,800 \mathrm{~kW}$. Careful consideration must be made of the operating characteristics of the biomass plant to optimize energy production, off set peak power consumption, integrate it with other generation such as wind power, and not waste fuel, both natural gas and wood chips.

Sustainable use of biomass, with good emission controls to reduce particulate matter, has many environmental advantages including net zero $\mathrm{CO} 2$, no SOx, and no mercury $(\mathrm{Hg})$ emissions. Ash residue can be recycled to farms and mixed with compost.

This biomass $\mathrm{CHP}$ district heat plan can be a primary source of heat and electric power for the Tribes Isabella Reservation. Integrating this system with wind power, solar hot water and other energy systems can provide the basis for cost effective $100 \%$ renewable energy to the Tribe.

\section{4: Natural Gas Engine Stand-by and Combined Heat and Power}

While a natural gas stand-by CHP system is not renewable energy, the electric O\&M staff indicated that the Tribes existing stand-by diesel electric generation cannot fully operate both the casino and the hotel in the event of a power outage. For this reason analysis of additional standby generation was undertaken. Our recommendation is that if additional stand-by generation is considered it should be a combined heat and power (CHP) natural gas engine set (preferably two engines) that can both 
generate electricity and heat during operation. These units can be started in a short period of time (30 minutes maximum for heat) and then replace the existing natural gas boiler heating capacity, without adding additional natural gas pipe line capacity to the SECR. These engine sets can also operate during peak electric demand periods, supplying domestic hot water to the SECR during any season, utilizing natural gas as efficiently $(85 \%-90 \%)$ with electricity and heat than the existing natural gas boilers. For example, one natural gas CHP engine can provide all of the domestic hot water for the SECR while generating electricity of roughly $1,400 \mathrm{~kW}$ electric capacity. While such system may be considered over-kill, if standby electric capacity is a critical concern, at a capital cost of approximately $\$ 2.9$ million for two engine sets, this plant has the following advantages:

- Provides stand-by and peak electric capacity of 3,500 to $4,000 \mathrm{~kW}$ depending on the number and size of engines selected.

- Using the same amount of present natural gas consumption, it will generate up to $25 \%$ of the SECR electricity and $2 / 3$ rds of heat, saving $\$ 500,000$ per year while having $100 \%$ SECR stand-by electric capacity.

- Provide a quick infrastructure basis for initiating the biomass CHP installation and district heat system, especially with the addition of a large heat storage system.

This option should be considered in conjunction with the other renewable energy options being examined. If, for example, the biomass $\mathrm{CHP}$ system is considered a priority, the natural gas CHP option may be downsized or eliminated all together. It should be noted however, that the timeline for this option is shorter than the biomass steam turbine, roughly one year versus two years. Cost and design details are provided in the appendix.

\section{5: Solar Thermal - Big and Small}

Solar hot water heating (solar thermal) is cost effective and should be considered. The system recommended is a flat plate collector (see appendix), typically consisting of 4 foot by 8 foot glass covered panels mounted in series and parallel to approximate the hot water heating loads. In mid-Michigan such systems can provide up to $50 \%$ of the annual domestic hot water heating requirement, while providing 90 to $100 \%$ of requirements during the spring, summer and early fall months.

Our analysis, utilizing solar data available from the US National Renewable Energy Laboratory for the MBS airport 30 miles east of the Tribe, determined the optimal size of a commercial system and small residential system. For the Soaring Eagle Casino Resort (SECR) we 
calculate that an optimal system should consist of solar hot water panels up to 200,000 sq. ft., mounted either on the upper level of the SECR parking deck, or in the adjacent parking or grass areas to the south. Our preference is on the parking deck with either an open or covered parking area under the panels. The following summarizes our analysis:

- Estimated cost: $\$ 4.8$ million

- Annual natural gas savings: $\$ 400,000$

- Large array to be installed, up to 200,000 sq. ft. on the parking deck or adjacent field near the SECR heating mechanical system to reduce heat distribution loss.

- Installation of a large hot water storage tank in with the system.

The following two photos (Figure 18) show the upper parking deck area where a large commercial solar hot water system can be installed at the SECR.

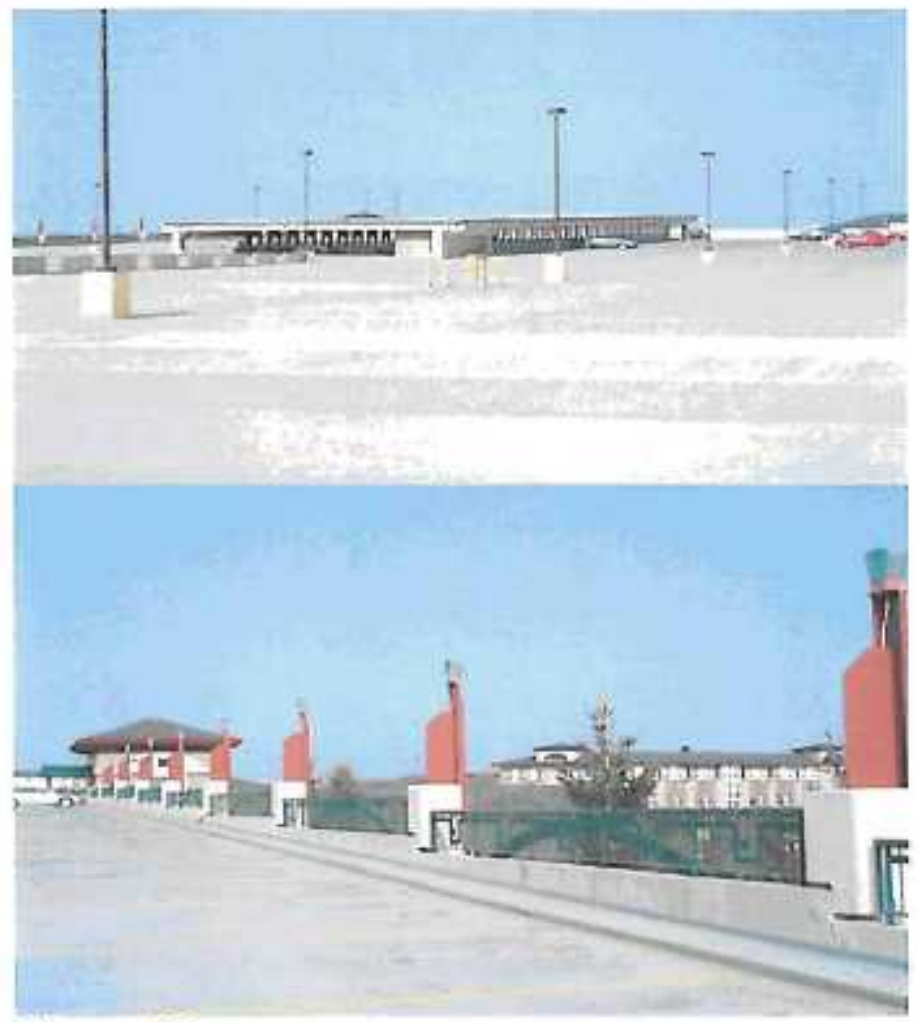

Figure 18

Solar hot water systems are recommended to be installed on all Tribal homes that have adequate solar access. While the size of the systems will vary slightly depending upon the individual family requirements, a family of three or four will typically require a $64 \mathrm{sq}$. ft. solar panel system, mounted south facing, tilted approximately 45 degrees on the roof or on the ground. Photos of a typical ground mounted system and 
heat exchanger and storage tank is shown here in Figure 19. A hot water storage system consisting of either the existing hot water heater, or preferably a secondary tank feeding directly to the existing hot water heater with a recommended capacity of 80 to 100 gallons. The efficiency of a solar hot water heat exchanger is directly related to the temperature difference (delta T) of the solar hot water storage system and the solar heat exchange fluid heated by the sun. Therefore, additional hot water storage in conjunction with the existing home hot water heater can provide a more efficient system and additional hot water supply.
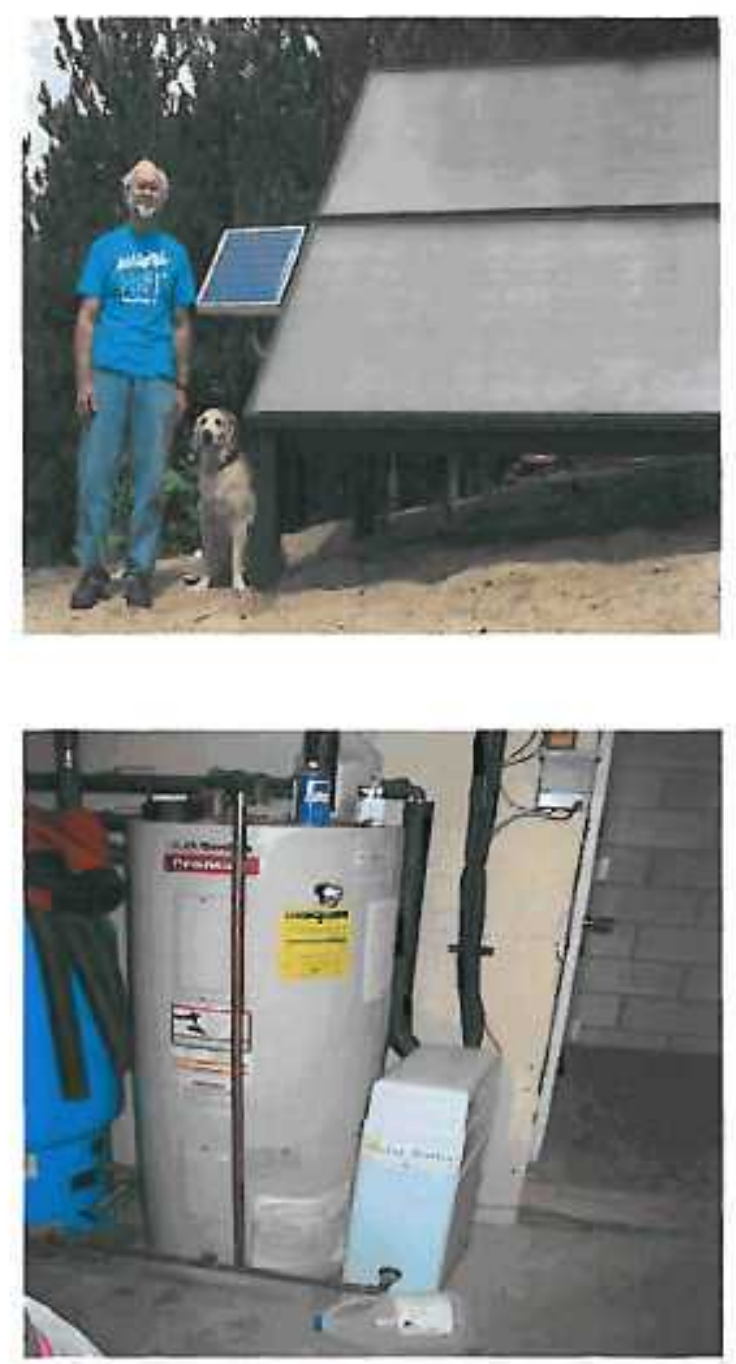

Figure 19

Depending upon the installation requirements of each individual home, the cost will range from $\$ 5,000$ to $\$ 7,000$, less personal income tax credits available from the federal government, up to $\$ 2,000$. The cost of the solar hot water will be competitive with the typical heat source in Tribal rural homes, either electricity or LP gas. Solar hot water heating systems such as those shown in the appendix are low maintenance and provide fixed price energy for the lifetime of the solar panels, 20 years or more. 
We recommend the Tribe public works department establish a solar hot water installation service or encourage Tribal members skilled in mechanical systems and service to establish a new major solar hot water commercial business in the region. In addition a financing program, with low interest loans, perhaps based at the Tribal Credit Union could be utilized to help spread the higher capital cost of the systems over time, such as with auto and home renovation loan.

\section{6: Solar Electric}

Solar electric (photovoltaic) systems, while extremely durable, reliable and low maintenance, especially when directly interconnected without battery storage systems, are not recommended for any significant electric power generation at the Tribe. We do recommend the Seventh Generation solar electric system discussed above. It also would be advisable for the Tribe to install a small, say $10 \mathrm{~kW}$ peak capacity system for experience and preparation for expanded solar PV systems as costs go down and incentive policies improve. The cost of a $10 \mathrm{~kW}$ system would be less than $\$ 100,000$.

The primary reason for this recommendation is due to the present high cost of these systems. Depending on the application the electric energy cost of a solar system in mid-Michigan solar conditions will range in the 50 to 60 cents per $\mathrm{kW}$-hr price. When the long-term, low maintenance, fixed cost electric energy from solar electricity is considered in contrast to increasing conventional electric costs, if reasonable financing is available, new policies and incentives can make this a serious option to consider.

One situation where we would recommend consideration of a solar electric system is for remote homes and facilities where high grid interconnection costs make the solar electric system cost effective. It can also be cost effective if a new facility is planned (such as a new hotel) where a solar electric system may be integrated into the new structure and budgeted in the new construction costs and long-term mortgage.

\section{7: Energy Storage, Controls, and Integration of Resources.}

Integration of the renewable energy resources and the installation of energy storage systems, particularly thermal (heat) storage and chilled water storage can optimize the systems and balance energy loads to achieve the goal of $100 \%$ renewable energy for the Tribe. 
As shown in the introduction of this report, the renewable energy systems analyzed, if all implemented in their full extent, can provide three to four times the present energy needs of the Tribe. Therefore, unless the Tribe is prepared to implement the "new vision" development plans in the near future (which require at least triple the present energy infrastructure and consumption), each system proposed should be downsized, integrated and optimized to provide the most economical, efficient and environmentally beneficial system possible. A computer controlied energy management system, with operations oversight, can adjust the renewable energy systems and plants according to the daily solar and wind resources available to the Tribe in accordance with the energy loads. The following graph, Figure 20, is a representation of two weeks (winter and summer) of how a mix of renewable energy systems, CHP systems and storage can cover the daily swings of energy consumption.
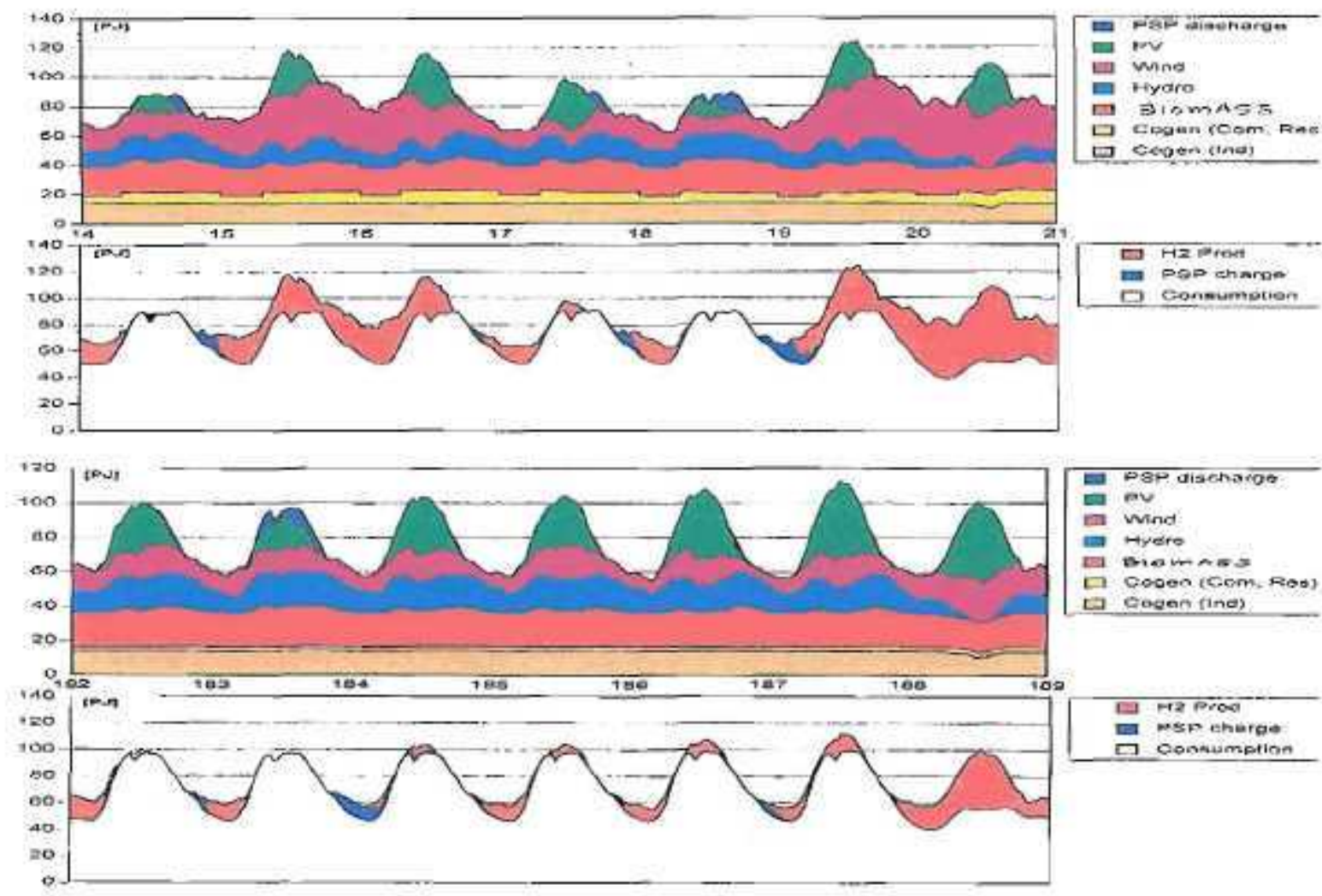

Figure 20

Once the Tribal Council moves forward with a decision to implement the new technologies, integrating the renewable energy systems, including energy storage, is the next frontier for the Tribe to analyze and consider. 


\section{8: Energy Efficiency}

Energy efficiency is almost always one of the most cost effective options for the Tribe to consider, particularly in older commercial and public facilities and homes. The Soaring Eagle Casino Resort, however, has unique operating conditions and entertainment qualities that make it difficult to change the efficiency of the operations. The hotel has a fourpipe heat and cooling system that provides efficient and high quality comfort, unmatched in most hotels. It is an excellently operated and managed facility as a world class resort.

While an energy efficiency study was beyond the scope of this project, an energy efficiency study was recently completed in 2007 by engineers from the Council of Energy Resource Tribes (CERT) and is available for review and consideration by the Tribe facility managers.

\section{0: Conclusions}

With a total Tribal energy bill for electricity and natural gas of $\$ 5.4$ million in 2007 , over $\$ 75$ million of capital cost investments in renewable can be justified. Put another way, in simple investment terms, if the Tribe could purchase a bond that would return $\$ 5.4$ million a year on the investment, how big of a bond would the Tribe be willing to buy? Renewable energy systems are like purchasing a secure, long-term bond.

With increasing electric and natural gas energy costs, wind and solar power with zero fuel costs, and biomass (wood chips, wood waste, etc.) with low fuel costs, provide the opportunity to minimize and basically fix energy fuel costs for the Tribe. What is required, however, is the investment in the capital costs for these renewable energy technologies in order to harvest the clean energy that all come from the sun.

The Tribal Council and administrative and operations staff must make a decision, if, how and when to proceed, based upon this menu of options. The next step will be detailed engineering and architectural plans to provide the basis for specific cost estimates and financing plans for the implementation of the selected options.

\section{0: Appendix}


Appendix A. Wind Conference Brochure 
Appendix B. Statement of Objectives 


\title{
Statement of Objectives \\ Saginaw Chippewa Indian Tribe \\ Wind/Alternative Energy Feasibility Study
}

\author{
Saginaw Chippewa Indian Tribe \\ 7070 E. Broadway \\ Mt. Pleasant, MI 48858 \\ Phone: 989-775-4015 \\ Fax: 989-772-4151 \\ Email: SKniffen@sagchip.org
}

July 28, 2004

DOE Grant Number DE-FG36-04GO14252 
DE-FG36-04G014252, A.000

Saginaw Chippewa Indian Tribe

\title{
Statement of Objectives
}

\author{
Saginaw Chippewa Indian Tribe
}

Wind/Alternative Energy Feasibility Study

\section{Background:}

The Saginaw Chippewa Indian Tribe is seeking to become self sufficient in their energy needs. Further, they wish to maintain their culture by protecting Mother Earth and looking ahead seven generations to preserve natural resources for the future. "Green" energy sources are the best way to accomplish both these objectives.

The Tribe's objectives are to provide power for its facilities and potentially create economic development and employment opportunities in its depressed areas, specifically Arenac County. Also utilizing renewable energy is consistent with the cultural heritage of the Tribe. Specifically, the use of renewable energy to provide power for the Seventh Generation grcenhouses, residential treatment center and proposed school is desired.

\section{Project Summary:}

The Saginaw Chippewa Indian Tribe is seeking funding from the D.O.E. to perform a feasibility stady on Tribal lands in Isabella County and Arenac County. The Tribe wishes to study the feasibility of windenergy, solar energy, geo-thermal energy, as well as other options that may be available. These efforts are to help the Tribe become sufficient in providing their own energy for the various buildings, cultural centers, schools and residences on Tribal land.

The study would include the initial program outline, through site selection, land agreements, wind assessment, environmental review, economic modeling, interconnection studies, permitting, sales agreements, financing, turbine procurement, construction contracting and operations and maintenance. If other alternative energy sources are more feasible parts of the scope of work will change to reflect investigation into the alternative sources and less on procuring a wind turbine.

\section{Tasks:}

a. Develop a detailed work plan for Tribe and DOE approval.

b. Order and install 2 meteorological towers and begin data collection.

c. Identify, select, and schedule work for subcontractors for cultural and avian issues.

d. Issue required progress and technical reports.

e. Conduct Wind \& Renewable Energy Conference, 
DE-FG36-04GO14252, A000

Saginaw Chippewa Indian Tribe

f. Engage local utilities and power marketers for interconncctivity power purchase agreements.

g. Prepare project economic performas with options.

h. Determine Tribal plans for development.

\section{Constraints to be considered}

a. Wind resource- if the wind resource is not sufficient to provide enough quantity to utilize wind turbines.

b. Market-the supply of energy may not be sufficient to market on the electric grid. There may be enough energy to supply the intemai needs of the Tribe.

c. Transmission Constraints-if the Tribe exports energy the existing power lines may not have the thermal/physical load to support the extra electricity. Also contractual transmission constraints may exist.

d. Community acceptance-if members of the community feel the visual impact is too severe, or a historical/cultural area is affected they may not wish to have the turbine located in that area.

e. Envitonmental-if an environmental constraint is insurmountable, e.g endangered species; avian flyways; negative visual impact. 
Appendix C. Small Wind Turbine Spec Sheets 


\section{Introducing the new VENTERA $12 \mathrm{~kW}$ Wind \& Solar Hybrid Electric System}

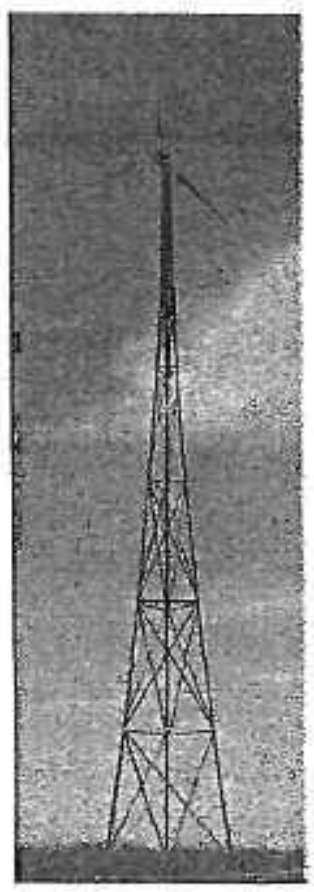

\section{Run Your Electric Meter Backwards!}

It doesn't get any simpler than this. This new concept in generating your own renewable energy performs much like any large electrical appliance such as a clothes dryer or air conditioner, but instead of taking electrical power FROM an outlet, you put electricity INTO the outletl If at any time your wind and solar system is producing more energy than you are using, your electric meter will run backwards reducing your electric bill. If your electrical loads are larger than the wind generator and solar production, your meter will slow down reflecting your reduced need to buy electricity at retail from the utility. No batteries are used or required! You get up to 12 kilowatts of hybrid renewable electricity, 10 kilowatts from the new Ventera VT10 wind turbine and 2 kilowatts from solar photovoltaics $100 \%$ of which goes directly to reduce 
vour electric bill by means of our exclusive dual input V112 synchronous inverter.

\section{Designed to Pay for Itself Quickly}

Few things in this life are free, but you might agree our new era Ventera V12 wind and solar system comes pretty close. Designed for at least a 30 year life and minimum maintenance, the Ventera wind turbine on an 80 foot tower can produce $\$ 2000$ or more worth of electricity per year, electricity you would otherwise have to buy, if your wind regime is $12.5 \mathrm{mph}-5.5 \mathrm{~m} / \mathrm{s}$ (class III) or better and you pay 8 cents or more for electricity. In not much more than 10 years you'll have earned back your original investment and in 30 years you could pocket 3 times or more the original investment! if you assume electric rates will go up in the coming years, and many predict they'll go up faster than the rate of inflation, you'il earn back your investment even faster. If you can depreciate the wind turbine cost as a farm or business expense, your payback will be even sooner. If your average wind speed is greater than $12.5 \mathrm{mph}-5.5 \mathrm{~m} / \mathrm{s}$, which is true for many sites in the US Midwest, your payback can be sooner yet. And, some states offer tax credits for small wind systems reducing the payback time even further. Careful design of every component and advanced tooling and manufacturing processes bring the initial cost of the Ventera turbine, synchronous inverter and self-supporting tower to historic lows. The frame is an aluminum casting (made from environmentally friendly recycled aluminum), not an expensive weldment. The downwind orientation eliminates the need for a costly and cumbersome tail. The blades are injection molded for low cost and absolute uniformity. The brushless, one-moving-part, large diameter alternator minimizes the use of copper, steel and rare earth magnet material. Our unique synchronous inverter utilizes a common power handling stage with separate wind and solar peak power tracking input stages to bring you hybrid grid connection at an unheard of low price.

\section{How the Ventera VT10 Wind Generator Works}

The new Ventera 10 kilowatt turbine is specifically designed to be the most efficient wind turbine available to convert wind energy into electricity. Three advanced, proprietary airfoilshaped blades "fly" in the wind, much like a modern airplane wing, to convert the kinetic energy of moving air mass into rotating mechanical force. The blades of this propeller have a specific shape which has been developed from over 30 years experience in wind turbine design to provide the highest output at the lowest noise level at all wind speeds for a propeller of this diameter.

Our patented (Number 7,186,083), exclusive governor automatically pitches the outer portion of each blade in response to a combination of wind force and propeller $\mathrm{rpm}$. This governor effectively limits propeller rpm to avoid damage to the propeller, generator, tower and electronics. This centrifugal-force activated blade pitching governor results in maximum power output in winds above governing speed as opposed to more primitive methods such as furling or stalling that result in steep reductions in power output in high winds. Our advanced governor will limit propeller rpm at much lower wind speed in the event of electrical load loss insuring 
always quiet operation.

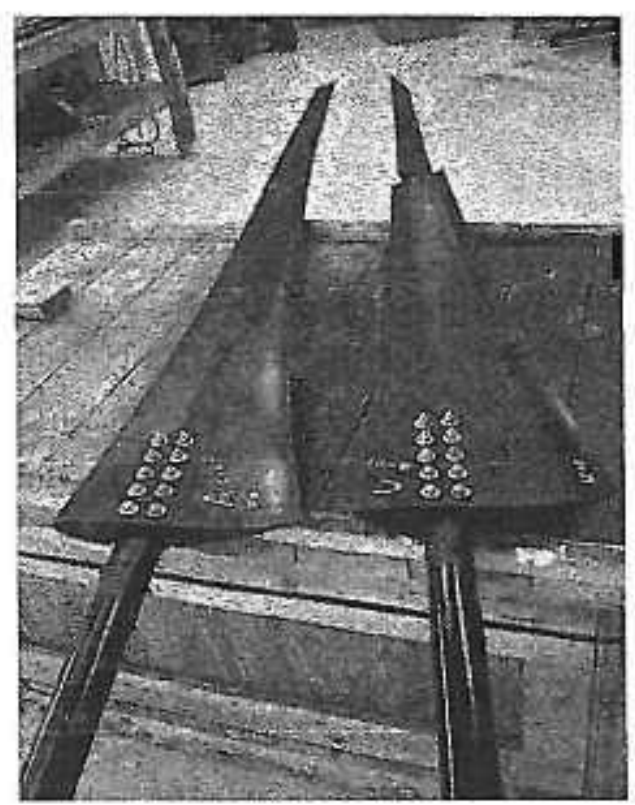

The rotating mechanical force of the propeller directly drives an ultra high efficiency permanent magnet generator mounted to an aluminum mainframe with no transmission to rob power, require lubrication or need maintenance. On the tower end of the mainframe is the vertical yaw shaft housing which also includes a redundant connection brush and slip ring assembly allowing the turbine to follow the changing wind direction without twisting the power output cable. Another function of the aerodynamic mainframe casting is to orient the wind turbine blades to the wind direction. In this modern, tailless, downwind design the wind first passes the tower and then flows over the propeller blades.

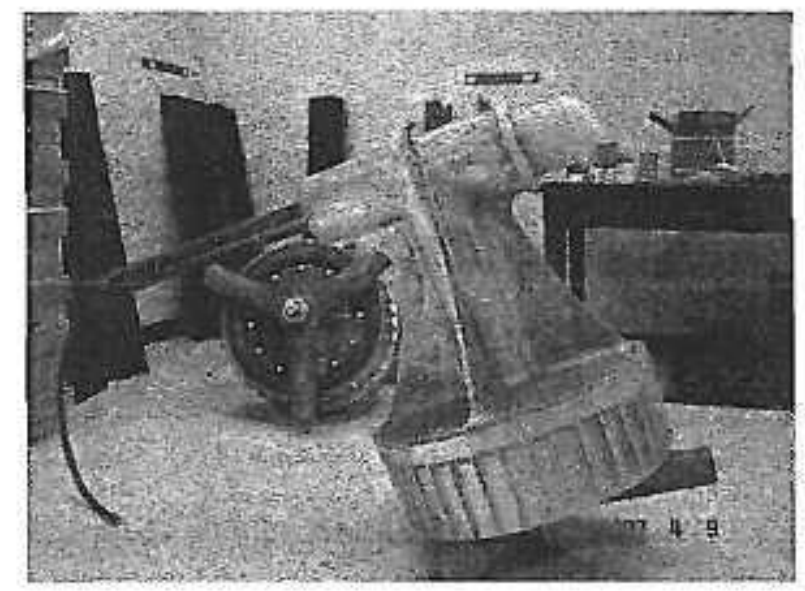

Electricity is generated by the Ventera generator in a high voltage, three phase form that 
minimizes the wire size and cost necessary to carry the electricity from the turbine to the inverter and your electric meter. This permits you to economically place the turbine and tower at a location on your property up to several thousand feet or 1000 meters from your electric meter which allows you to choose the tower site on your property with maximum exposure to the wind.

The advanced generator features oversize rare earth super strength magnets rotated by the propeller inside an extra large, stationary copper winding assembly. By over sizing these components and choosing the highest grade steel for the winding laminations, Ventera achieves a superior generator efficiency averaging over $90 \%$ resulting in more kilowatt hours of electrical energy per month for this size propeller. There are no electronic components on top of the tower to require inspection or repair or to be prone to damage from lightning or static electricity.

\section{Ventera VI12 Synchronous Inverter}

From the wind generator and tower and the solar panels the electricity flows to the Ventera VI12 Synchronous inverter normally located near your circuit breaker box and electric meter. The inverter has two separate inputs, one for wind and one for solar. Each input has its own "peak power tracking" algorithm controlled by the inverter microprocessor. The wind input is programmed to control the propeller rpm to its optimum value for every wind speed, thus maximizing the turbine efficiency of converting wind energy to electricity. This Ventera exclusive feature alone increases monthly kilowatt hour production by up to $15 \%$ over other wind turbine-synchronous inverter combinations.

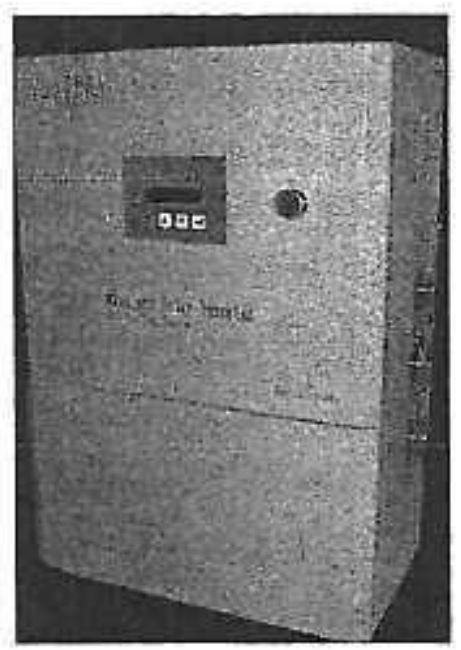

The solar input has a separate peak power tracker that maximizes the available solar electricity even early in the morning or late in the evening or on cloudy days. The Ventera inverter has been designed to have the highest efficiency in the industry assuring you of maximum energy available to reduce your electric bill. This efficiency at full $12 \mathrm{kw}$ output is 
$94 \%$ or better. Another unique feature of our inverter is that electrical consumption has been minimized when the inverter is idle or when no solar or wind input is available, for example at night when the wind is caim.

A key function of the inverter is to automatically disconnect from the utility in the event of utility failure to prevent possible back feeding of the utility line and any hazard to workers on the utility line. The inverter will detect an out-of-frequency or out-of-voltage line condition and disconnect immediately meeting requirements of the national electric code. The UL/CSA approval sticker on the inverter assures your electrician and utility of compliance with this important requirement.

You will appreciate the full monitoring features we've included with our new inverter. The front panel readout provide continuous readout of power in kilowatts, wind and solar energy in kwh since last reset and total $\mathrm{kwh}$ since installation plus convenient diagnostic information. For solar PV only systems we also offer a $12 \mathrm{kw}$ solar PV version of the new synchronous inverter.

\section{Ventera VSST Self Supporting, Fold Over Tower}

No one wants unsightly guy wires in the yard to interrupt mowing, grazing, volleyball or gardening, and Ventera Energy has designed a rugged, good looking three-legged, selfsupporting tower to complement the Ventera V12 wind turbine with a beauty and grace you'll be proud to have on your homestead. A specially designed die-formed leg gives the tower a graceful curved taper reminiscent of the Eiffel Tower in Paris. It will remind you of the common windmill tower seen pumping water around the world, but you and your neighbors will also immediately recognize the exclusive, Ventera style. Towers come painted in weather-resisting black enamel to enhance the beauty of the silver and black colors of the Ventera turbine.

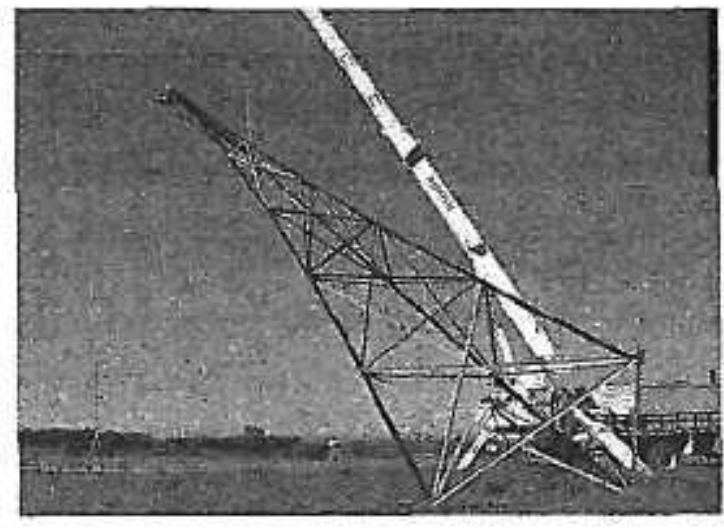

The tower is offered in heights of $35,50,60,70$ and 80 feet and comes complete with assembly hardware, tower top to bottom wiring harness, junction box and mounting hardware for the Ventera VT10 wind turbine. The tower is shipped in bundles by truck to your door and is designed for easy owner assembly following the included instructions. Each leg, vertical brace 
and horizontal brace is clearly labeled according to its position in the assembled tower.

The tower is hinged at the base and the hinged base plates that go over your anchor bolts are included. Each base plate is provided with a leveling arrangement to assure the tower is perfectly plumb. Users with a farm tractor can design a gin pole system for raising and lowering the tower and turbine for ground level service. Others will use a crane service to raise and lower the tower.

\section{About Ventera Energy Corporation}

Three principals, each with over 30 years experience designing and manufacturing small wind turbines, solar hot water systems, solar PV systems and high performance glazing systems, teamed together to form Ventera (the new era of wind) Energy Corporation January 1st, 2004 with the goal of designing, proving and manufacturing an all-new wind and solar hybrid renewable energy system that would be the first ever cost effective small wind system for the moderate wind regimes where most people live. Our experience includes design and manufacture of the WhirlWind line of 1000 to 4000 watt wind generators and the Whisper line of 500 to 4500 watt turbines sold to and now manufactured by Southwest Windpower. After 3 years of design, prototyping and field testing, Ventera is proud to present to the world our all-new 10 kilowatt VT10 Wind Turbine, our exclusive taper leg self-supporting tower and the first ever dual wind and solar input, dual peak power tracking 12 kilowatt VI12 Synchronous Inverter. In wind regimes having $12.5 \mathrm{mph}-5.5 \mathrm{~m} / \mathrm{s}$ or better average wind speeds at 30 feet (10 meters elevation), we have achieved our goal of a 10-year payback for many if not most potential users. 


\section{NorthWind ${ }^{\oplus} 100$ Wind Turbine}

d. Distributed 


\section{Distributed Energy Systems' NorthWind 100 wind turbine provides cost-effective,} highly reliable renewable energy in demanding environments worldwide.

Desigried specifisaly for isolated grid and eistributed generation applicatrons, the Northwind 100 wind iurbens 5 a sta:e-0 -he-art vi tage.scale wind unbine D strubuted Energy Systems has drown an 30 years of experience

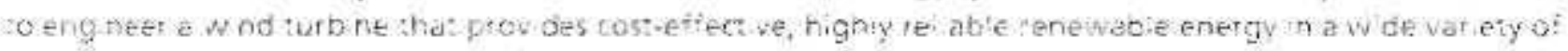

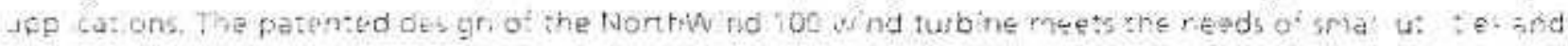
Whatendent pover produsers.

\section{bey ffatures}

Simplicity

High reliability and low maintenance were the focus in developing the Northwind 100 wind turbine. The design integrates industry proven robust components with innovative design features to maximize wind energy capture in rura!, remote and harsh environment locations. The Northwind 100 wind turbine features a minimum of moving pars and vital subsystems to deliver high system availability. The uncomplicated rotor design allows safe, efficient turbine operation.
Serviceability

Our sophisticated remote monitoring and control software allows real-time accessibility of the turbine thus minimizing unnecessary service calls. When a site visit is required, all service activities can oscur within the tubular tower or heated nacelie hotsing, providing complete protection from harsh or unpredictable weather conditions. Designated work areas provide ample room to perform service activities.
Power Quality

The NorthWind $\$ 00$ wind turbine provides reliable power in distributed generation and village systems where the power grid is typicaliy "soft and unbalanced." Our syn. chronous, variable speed, permanent magnet, direct drive generator and integrated power convertet increase energy capture while eliminating current inrush during control transitions. This turbine can be connected to large power grids and remote wind diesel configurations without inducing surges, effectively providing grid support rather than compromising it.
NorthWind 100/21 Wind Turbine Power Curve Standard Density

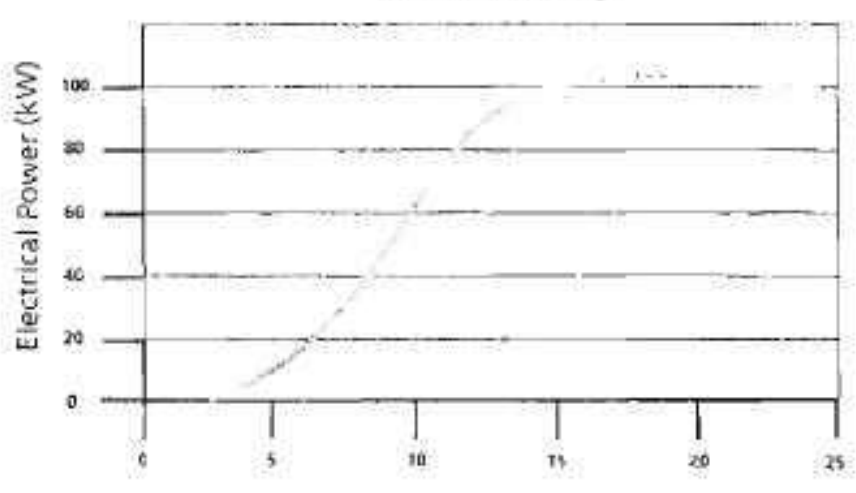

Wind Speed at Hub Height (m/s)
NorthWind 100/21 Wind Turbine

Annual Energy Production

Standard Density, Rayleigh Distribution

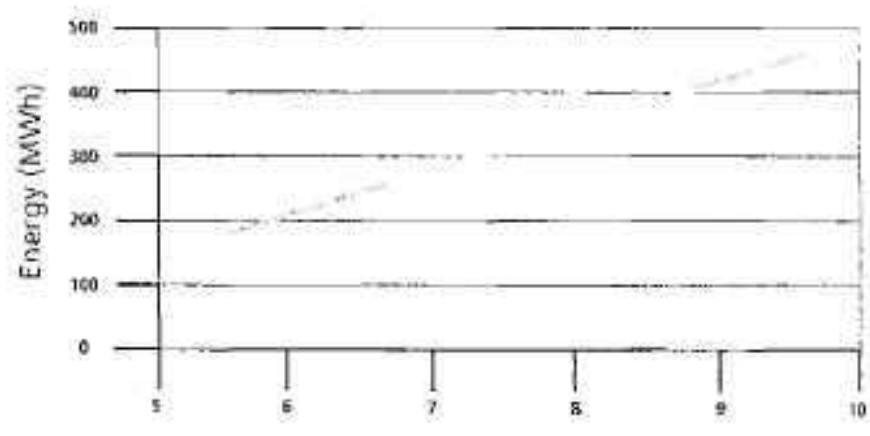

Amnual Average Wind Speed at Hub Height (m/s) 
- Three fiberglass reinforced plastic blades bolted to a rigid hub that mounts directly to the generator shaft eliminates the need for rotating blade tips, blade pitch systems and speed increasing geatboxes.

- Variable speed, permanent magnet, direct drive gener ator/converter system is tuned to operate the rotor at the peak performance coefficient, and also allows stall point rotor control to contend with wide variation in air density found in the target applications.

- Safety system provides both norma shutdown and emergency braking backup functions.

- Advanced power converter features setpoint control of power factor and/or VAR5.

- Web-based SmartViewo remote monitoring system also available.

NorthWind 100 Wind Turbine Technical Specifications

\begin{tabular}{|c|c|}
\hline Turbine Design Class & IEC NTGS Class S \\
\hline Detign Standord & Compitant with itc $51400-3$ \\
\hline Aated Power & $100 \mathrm{kw}$ \\
\hline Power Aegulstion & Variable speed stall \\
\hline Rotor Diameters & $19 \mathrm{~m}, 20 \mathrm{~m}, 2 \mathrm{~mm}$ \\
\hline Hub Heights: & $25 \mathrm{~m}, 30 \mathrm{~m}$ \\
\hline Yaw System & Attive upvend \\
\hline Turbise Electrical Dutput & 48OVAC, 3 phase, $50 \mathrm{bOHz}$ \\
\hline Grid Tolerance. & - $10 \mathrm{~d} \cdot 15 \%$ voltage, $+\mathrm{H}-2 \mathrm{~Hz}$ \\
\hline Grid interface & 115kva yaniformer (spec ovalahte) \\
\hline Operating Temperature & $40^{\circ} \mathrm{C}$ to 50 oc \\
\hline Lightring Protection & Cormpliant with ite $61024-1$ \\
\hline Icing: & $10.30 \mathrm{~mm}$ \\
\hline
\end{tabular}

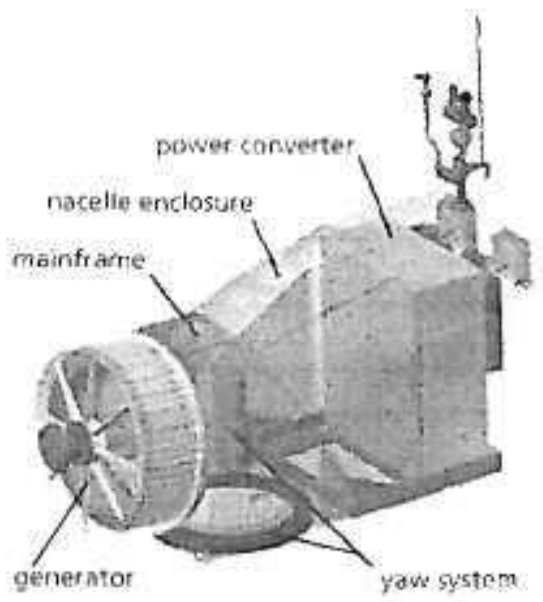

Nacelle assembly

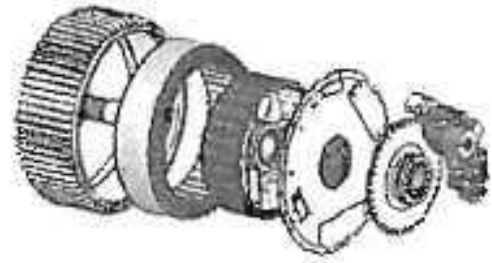

Passively-cooled, permanent magnet, direct drive generator eliminates the drivetrain gearbox and maximizes energy copture

\section{easasimitis}

\section{Wind-Diesel Systems in Remote Alaska}

Distributed Energy Systems successfully installed and commissioned three new NorthWind 100 turbines in the community of Kasigluk, Alaska. As part of a larger wind-diesel energy initiative by Alaska Village Electric cooperative (AVEC), these turbines will produce approximately 675,000 kWh annually, By displacing $32 \%$ of the energy normally generated by diesel fuel, the new systems are expected to generate a potential savings of over $\$ 95,000$ per year. 


\section{Contact us}

Corporate Headquarters

Hydrogen Generation; Technology Generation

10 Technologv Drive

Waltingford, CT 06492

TEl +01 203678.2000

Power Generation

29 Pitman Raad

Barre, VT 0564?

$T \in I+01802.4612955$

Visit ws oriline at

whw distributed energy cam

NASDAO-DESS

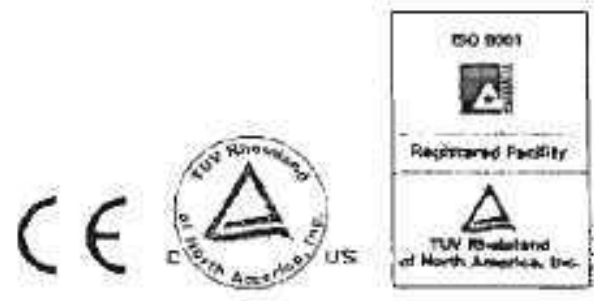

6. 2007 Destributed Eritegr Systems Corp, All Rights Rererved H(1) 0600 0053 0007

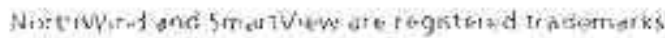

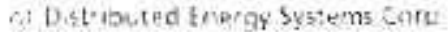




\section{NorthWind ${ }^{\oplus} 100$ Wind Turbine}




\section{Distributed Energy Systems' NorthWind 100 wind turbine provides cost-effective,} highly reliable renewable energy in demanding environments worldwide.

Desicred specifically for isolated grod and distributed generation applicatons, the Northwind 100 wind turtine

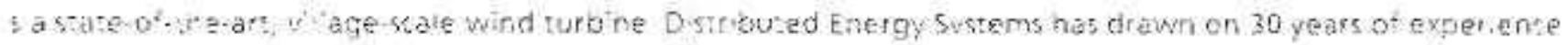
to eng neer a whd turb ne the: provides costeffect ve, highy re abe renewabie anergy n a wide var ety of

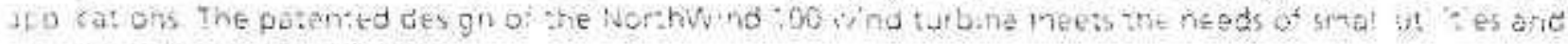
Weperders poviri pioducert.

\section{thing teatures}

Simplicity

High reliability and low mainte. nance were the focus in developing the NorthWind 100 wind turbine. The design integrates industry proven robust components with innovative design feazures to maximize wind energy capture in rural, remote and harsh environment locations. The NorthWind 100 wind turbine features a minimum of moving pars and vital subsystems to deliver high system availability. The uncomplicated rotor design allows safe, efficient turbine operation.
Serviceability

Our sophisticated remote monitoring and control software allows real-time accessibility of the turbine thus minimizing unnecessary service cails. When a site visit is required, all service activities can occur within the tubular tower or heated nacelle housing, providing complete grotection from tiarsh or unpredictable weather conditions. Designated work areas provide ample room to perform service activities.
Power Quality

The Northwind 100 wind turbine provides reliable power in distributed generation and village systems where the power grid is typically "soft and unbalanced." Our synchronous, variable speed, permanent magnet, direct drive gener ator and integrated power converter increase energy capture while eliminating current incush during control transitions. This turbine can be connected to large power grids and remote wind-diesel configurations without inducing surges, effectively providing grid support rather than compromising it.
NorthWind 100/21 Wind Turbine Power Curve Standard Density

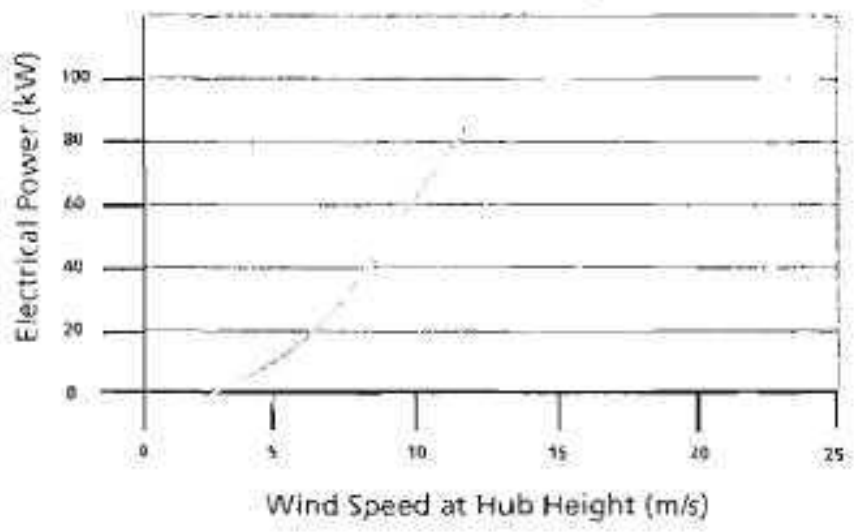

NorthWind 100/21 Wind Turbine Annual Energy Production Standard Density, Rayleigh Distribution

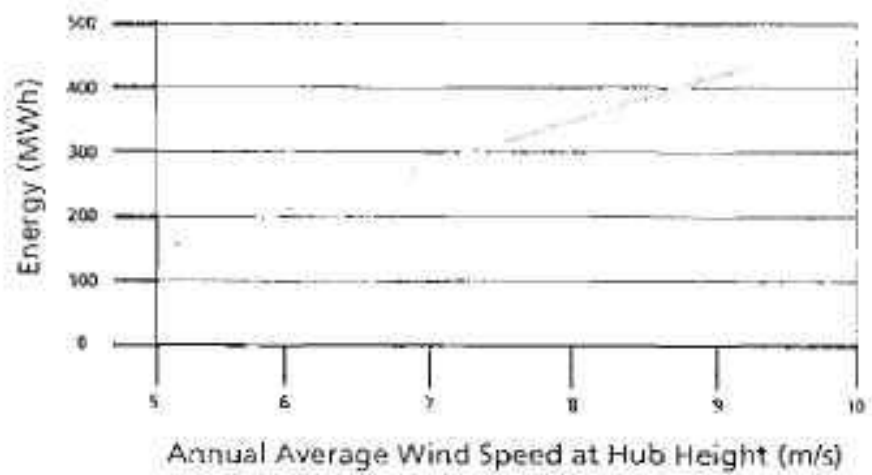


- Three fiberglass reintorced plastic blades bolted to a rigid hub that mounts directly to the generator shaft eliminates the need for rotating. biade tips, blade pitch systems and speed increasing gearboxes.

- Variable speed, permanent magnet, direct drive generator/converter system is tuned to operate the rotor at the peak performance coefficient; and also allows stall point rotor control to contend with wide variation in air density found in the target applications.

- Safery system provides both normal shutdown and emergency braking backup functions.

- Advanced power converter features setpoint control of power factor andior VARs.

- Web-based SmarView remote monitoring system also available.

NorthWind 100 Wind Turbine Technical Specifications

\begin{tabular}{|c|c|}
\hline Turbine Designclass & IEC WrGS Class: 5 \\
\hline Deslyn: Stondord & Compliart with IEC Gino0-1 \\
\hline Rated Power & $100 \mathrm{kN}$ \\
\hline Pewer flegulacion & Variabie speed stall \\
\hline Rotor Dismeters & $19 \mathrm{~m}, 20 \mathrm{~m}, 21 \mathrm{~m}$ \\
\hline Hub Heights & $25 \mathrm{~m} 30 \mathrm{~m}$ \\
\hline Yaw System & Active upwind \\
\hline Turbine Electrical Dutpat & 480VAC. 3 phase, $50 \mathrm{O} 60 \mathrm{~Hz}$ \\
\hline Grid Tolerance & $+10 \mathrm{H} 15 \%$ rollage: $4 /-2 \mathrm{~Hz}$ \\
\hline Gid Intertsce & $175 \mathrm{kva}$ traristormer (spec avalable) \\
\hline Opesating Tenyperature: & $-40 \times 51050 \times 5$ \\
\hline Lightning Protestion & Compliant with IEC 61024-1 \\
\hline king & $1030 \mathrm{mn}$ \\
\hline
\end{tabular}

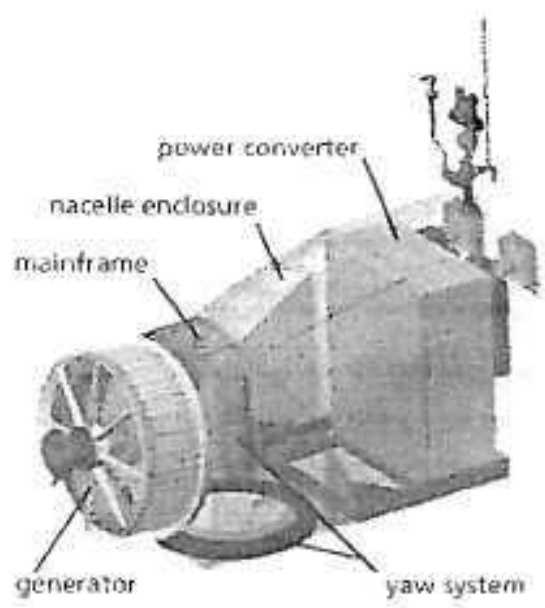

Nacelte assembity

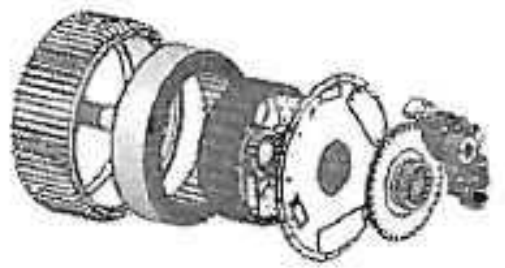

Passively-rooled, permanent magnex, direct drive generator eliminates the drivetrain geasbox and maximizes energy capture.

\section{cosing}

\section{Wind-Diesel Systems in Remote Alaska}

Distributed Energy Systems successfully installed and commissioned three new Northwind 100 turbines in the community of Kasigluk. Alaska. As part of a larger wind-diesel energy initiative by Alaska Village Electric Cooperative (AVEC), these turbines will produce approximately 675,000 kWh annually; By displasing $32 \%$ of the energy normally generated by diesel fuel, the new systems are expected to generate a potential savings of over $\$ 95,000$ per year.

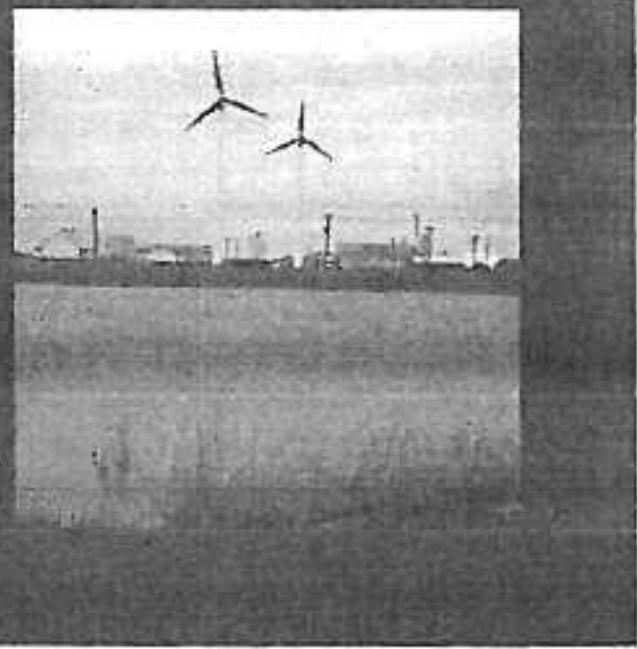




\section{Contact us}

Corporate Headquarters

Hydrogen Generation I Terbnology Gener auon

10 Technology Drve

Wallingtord, CT 06492

Tel $\$ 01203678.2900$

Power Gentration

29 Pitman Riad

Garre, VT 05641

Tel +018024512955

Visit us online at

WWw distributed-energycems

NASDAO DESC

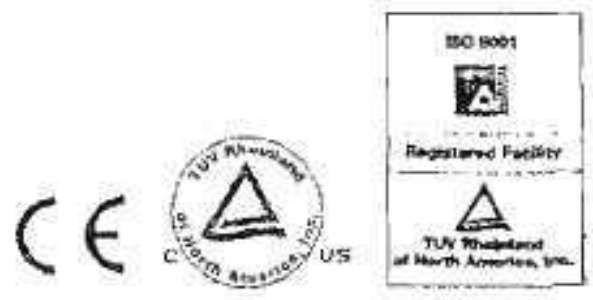

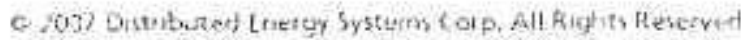
etaking 00530407

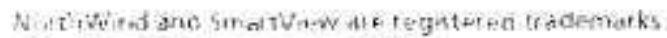


Saginaw Chippewa Casino Resort

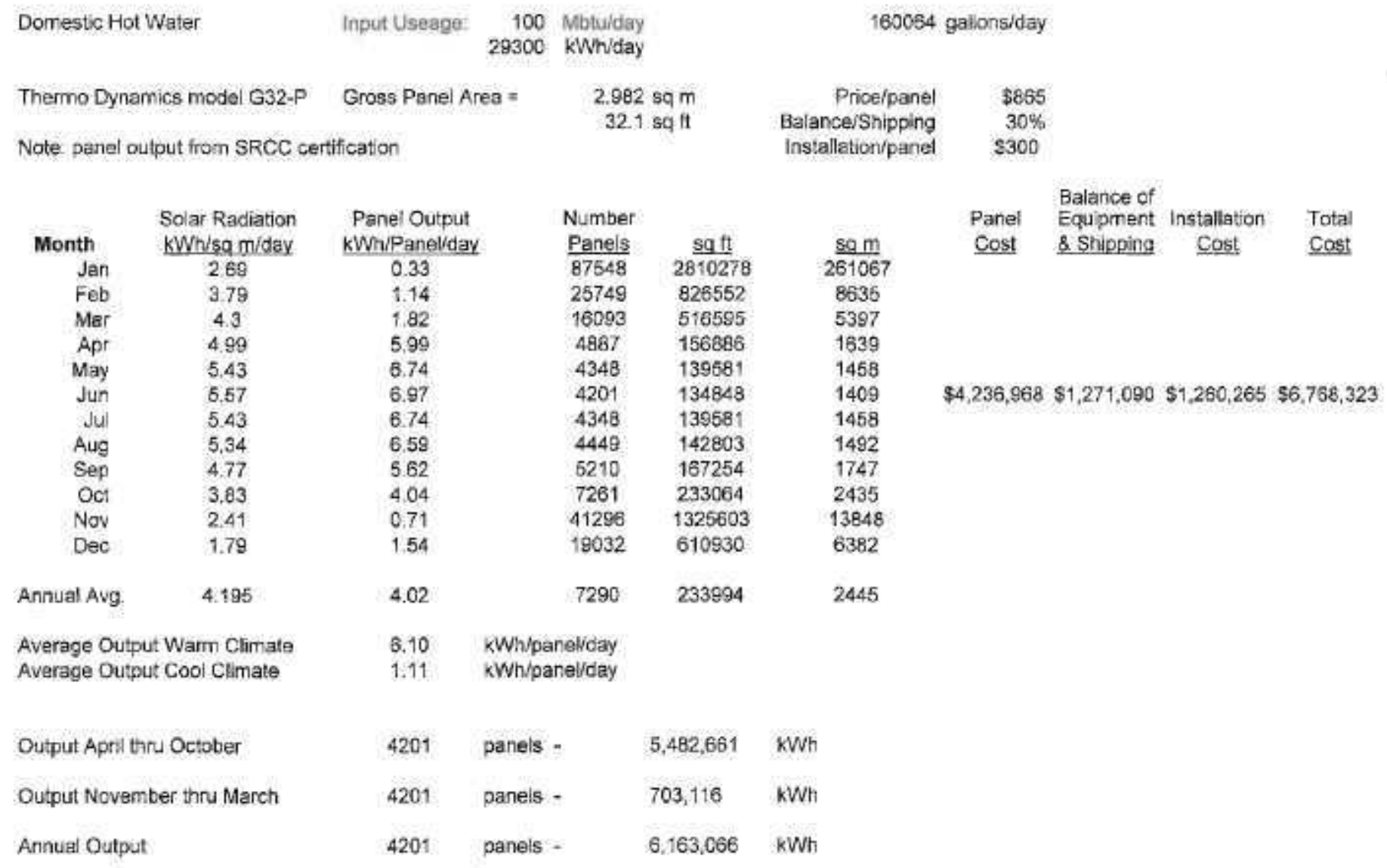


Saginaw Chippewa - Residential

7/17/08

Domestic Hot Water $\quad$ input Useage: $\quad 80$ gallons/day $\quad \begin{array}{r}49980 \text { Btu/day } \\ 14.6 \mathrm{kWh} / \mathrm{day}\end{array}$

Panel - Thermo Dynamics model G32 Gross Panel Area = $\quad 2.982$ sq $\mathrm{m}$ $32.1 \mathrm{sq} f \mathrm{ft}$

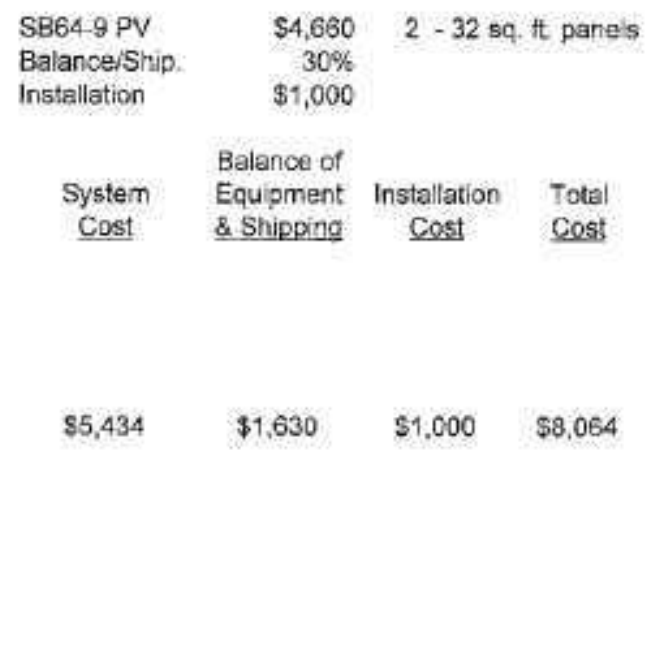

\begin{tabular}{|c|c|c|c|}
\hline Panel Output & Number & & \\
\hline kWh/paneV/day & Panels & sqft & $\mathrm{sg} \mathrm{m}$ \\
\hline 0.33 & 43.8 & 14046 & 130.5 \\
\hline 1.14 & 129 & $4 \uparrow 3.1$ & 38.4 \\
\hline 1.82 & 8.0 & 258.2 & 24.0 \\
\hline 5.98 & 2.4 & 78.4 & 7.3 \\
\hline 6.74 & 2.2 & 69.8 & 6.5 \\
\hline 6.97 & 2.1 & 67.4 & 6.3 \\
\hline 6.74 & 2.2 & 69.8 & 6.5 \\
\hline 6.68 & 2.2 & 71.4 & 6.6 \\
\hline 5.62 & 26 & 83.6 & 7.8 \\
\hline 4.04 & 3.6 & 116.5 & 10.8 \\
\hline 0.71 & 20.6 & 682.5 & 81.6 \\
\hline 1.64 & 9.6 & 305.3 & 28.4 \\
\hline 4.02 & kWh/paneliday & & \\
\hline 3,080 & kWryear & & \\
\hline
\end{tabular}

Annual Average Output per day

Annual Output 2 panels

\begin{tabular}{cc} 
Month & $\begin{array}{c}\text { Solar Radiation } \\
\text { kWh/sa m/day }\end{array}$ \\
\cline { 2 - 2 } Jan & 2.69 \\
Feb & 3.79 \\
Mar & 4.3 \\
Apr & 4.99 \\
May & 5.43 \\
Jun & 5.57 \\
Jul & 5.43 \\
Aug & 5.34 \\
Sep & 4.77 \\
Oct & 3.83 \\
Nov & 2.41 \\
Dec & 1.79
\end{tabular}

ation

Note: panel outout from SRCC certification

3,080 


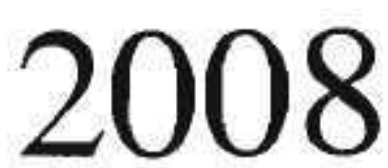

JANUARY 2008
101 Frazee Avenue - Dartmouth Nova Scotia $\cdot$ Canada $\cdot$ B3B-1Z4

Tel: (902) 468-1001 - Fax: (902) 468-1002

solarinfo@thermo-dynamics.com

www themo-dynamics com

\begin{tabular}{|l|l|r|}
\hline \multicolumn{2}{|c|}{ R E T A I L Price List } \\
\hline Product \# & Description & Retail \\
\hline
\end{tabular}

\begin{tabular}{|c|c|c|}
\hline \multicolumn{3}{|c|}{ Solar Collectors } \\
\hline $\mathrm{G} 32-\mathrm{P}$ & $4^{\prime} \times 8^{\prime}$ grid, $3 / 4^{\prime \prime}$ headers, semi-seiective painved surface & 864 \\
\hline$G 32-5 P^{*}$ & $4 \times 8^{\prime}$ grid, $3 / 4^{\prime \prime}$ headers, selective surfaco & 976 \\
\hline$G \times X$ & 1" header - additional charge & 83 \\
\hline GU34 & 3/4" unions installed on grid collector - 2dditional charge & 32 \\
\hline$\$ 32 A-P$ & $4^{4} \times 8$ serpentine, semi-selective painted surtace & 863 \\
\hline $5328 \cdot \mathrm{P}$ & $4^{\prime} \times 8^{\prime}$ serpentine, semi-selective painced surface & 852 \\
\hline$\$ 32 A-\$ P *$ & $4^{\prime} \times 8^{\prime}$ serpentine, selective surface & 962 \\
\hline $532 B-S P+$ & $4^{\prime} \times 8$ serpentine, selective surface & 950 \\
\hline ST or GT & $4^{\prime} \times 8-4$ thick collector addirional charge & 883 \\
\hline
\end{tabular}

\begin{tabular}{|c|c|c|}
\hline \multicolumn{3}{|c|}{ Collector Rack Klts } \\
\hline $\mathrm{K} 1000$ & Galvanized strut. 1-5/8", per foot (Unistrut) & 4.39 \\
\hline K1002 & Galvanized strut - single tier upright - for preched roof & 219 \\
\hline $\mathrm{K} 1004$ & Galvanized strut - single tier upright $-45^{\circ}$ - ground or flat rool & 224 \\
\hline K1006 & Gabranized strut - single tier upright - $60^{4}$ - ground or flat roof & 231 \\
\hline K1050 & Flush mount kis (for I G or S collector) & $3 t$ \\
\hline X10525 & Single colfector pinch clip, ofw spring nus and ss bolt & 5.49 \\
\hline K10520 & Double collector pinch clip, ciw spring nut and ss bolt & 7.94 \\
\hline K1055 & Fhush mount kit (for 25 collectors) & 63 \\
\hline K1056 & Flat raof kit (for 25 collactors) & 323 \\
\hline K1057 & Flat roof kit (for I S collactor) & 153 \\
\hline K105B & Inclined rool kit (for 25 collectors) & 277 \\
\hline K 1059 & Inclined roof kit (for I S collector) & 130 \\
\hline K1060 & PV module mounting kit (mounts PV20 to collector) & 29 \\
\hline
\end{tabular}

\begin{tabular}{|l|l|l|}
\hline Solar Boiler TM $^{\text {Module }}$ & \\
\hline SBM-9DC & Solar Boller TM module (12 VDC) & 1777 \\
SBM-13DC & Solar Boller Tw modula MAG DRIVE (12 VDC) & 1921 \\
\hline
\end{tabular}

\begin{tabular}{|l|l|r|}
\hline PV modutes and LCB & 528 \\
\hline PV20 & 20 watr PV module & 754 \\
PV30 & 30 watr PV module & 132 \\
\hline DTB & Delta-T Booster (Sersors not included) & 132 \\
\hline
\end{tabular}

\begin{tabular}{|c|c|c|}
\hline \multicolumn{3}{|c|}{ Solar Boiler is Installation Kits } \\
\hline K2030-50 & Copper ube kin, s0' $\left(3 / 8^{\prime \prime} \& 3 / 8^{\prime \prime}\right.$, & 403 \\
\hline K3015 & Copper tube interface kit (3/8" CT to $1 / 4^{-1}$ BHU) & 11 \\
\hline K3035 & Copper tube splicing kdt (for one $3 / 8^{\prime}$ and one $1 / 4^{\circ}$ CT) & 16 \\
\hline K3036 & Copper tube splicing kis (for one $3 / 8^{\prime \prime}$ and one $3 / 8^{\circ}$ CT) & 16 \\
\hline K 4000 & Solar Soiler PRV upgrade kit & 38 \\
\hline CT6-100 & Copper tube $\left(100^{\circ} \times 3 / 8^{\prime \prime}\right)$ & 209 \\
\hline LVTIB/4 & PV and Sesnor Wire, 18 gauge, 4 conductor, per foot & 1.66 \\
\hline CTI & Pipe insulation $3 / 8^{\circ}$, per $6 \mathrm{ft}$ length & 7.98 \\
\hline
\end{tabular}

Prices do not include GST, HST, \& PST. Prices are FOB Dartmouth, Nova Scotia. Canaela.

Prices are subject to change without notice. Prices are in Caradan dollars.

- Limited quantitles aveilable. For repairs onily.

* Built-in stroiner not ovallable for this pump model

Visit us on the web at: www.thermo-dynamics.com Email us at: solarinfo@thermo-dynamics.com 
PRICE LIST (Recail)

\begin{tabular}{|c|c|c|}
\hline Product * & Description & Retall \\
\hline & & 5 \\
\hline \multicolumn{3}{|l|}{ Storage Tanks } \\
\hline TG180 & Tank - glass lined (1 BO litar glass lined) & 579 \\
\hline TG270 & Tank - folss lined (270 liver glass llned) & 752 \\
\hline \multicolumn{3}{|c|}{ Miscellaneous Hardware } \\
\hline GLYUSP & Propylene glycol, USP pura (\$Nlitro) & 10.78 \\
\hline GLYUSM & Propylens glycol, USP mixed $40 / 60$ wich Distilled water (Sflicre) & 6.38 \\
\hline GL-30 & Solar controller ( 110 VAC) & 224 \\
\hline SAS-10 & Thermistor ternperature sensot (10,000 ohm) & 20 \\
\hline MGE & 120 VAC motor ( $t / 12 \mathrm{hp}, 125 \mathrm{watss})$ & 102 \\
\hline PIS42F & Grundios circulator (15 f(-2) USCPM) & 175 \\
\hline PS1542F & Grundfos circulacor (3 Speed) & 174 \\
\hline PI5Iasu & Grundios circulator SS $(7 \mathrm{fr}-11$ USGPH) $\mathrm{d} / \mathrm{w}$ unions & 294 \\
\hline P26998F & Grundfos circulator Bronze ( 30 fo- 35 USGPM) & 467 \\
\hline PFL & Flanges, pzir (POOB or $P 009$ or $P 1512$ or $P 2699 F)$ & 29 \\
\hline
\end{tabular}

\begin{tabular}{|c|c|c|}
\hline \multicolumn{3}{|c|}{ Solar Pumps tw \& accessories } \\
\hline$P 24070$ & Solar Pump ${ }^{20}$, (Pump/motor/LCB) 24 L/min - 70 GPH & 619 \\
\hline P50140 & Solar Pump ${ }^{\text {na }}$, (Pump/motornLCB) $5.0 \mathrm{~L} / \mathrm{min}-140 \mathrm{GPH}$ & 642 \\
\hline PII8330"e & Solar Pump ${ }^{m}$, (Pumpimotor/LCB) 11.8 L/min - 330 GPH & 731 \\
\hline P24070mph & Solar Pump ${ }^{n 4}, P 24070$ - Magnetic Drivo - built-in strainer $n / 2$ & 684 \\
\hline P50140M"* & Solar Pump NM, P50140 - Magnetic Drive - built-in surainer n/s & 692 \\
\hline BPOIS & Pump (brass, rotary vane) clw built-in strainar (replaces 1526) & 215 \\
\hline BPO70 & Pump (brass, rotary rane) clw bullt-ln strainer (rephaces 1505) & 215 \\
\hline$B P 140$ & Pump (brass, rocary vane) ofw built-In stralner $140 \mathrm{GPH}$ & 240 \\
\hline BP330 30 & Pump (brass, rocary vana) $330 \mathrm{GPH}$ - built-in strainer n/a & 332 \\
\hline BPOTOMN: & Pump (brass, rocary vano) 70 GPH - Magnedic Drive & 306 \\
\hline BPI $40 \mathrm{M}=$ & Pump (brass, rocary vane) 140 GPH - Magpedic Drlve & 322 \\
\hline MPS-DCM+4 & Pump/motor set (includes MDCF and BPO70M) & 552 \\
\hline MPS-DC & Pumpimotor set (includes MDCV and BPQ70) & 469 \\
\hline MDCV & OC motor, ( $1 / 15 \mathrm{hp}, 20$ wacts), V-band mount & 255 \\
\hline MDCF & DC mocor, (1//5 hp, 20 waves), flange mount. & 223 \\
\hline RH-1 & Rubber Hoses olw $1 / 2^{2}$ female swivel firting pair & 29 \\
\hline PSTIO0 & Replacemenc strainer for PIOS86 \& P1321 (100 mesh) & 4 \\
\hline
\end{tabular}

\begin{tabular}{|c|c|c|}
\hline \multicolumn{3}{|c|}{ Solar Glass } \\
\hline GL24. & Solar glass $\left(34^{\prime \prime} \times 96^{\prime}\right)$ tempered & 152 \\
\hline GL25* & Solur glass $\left(46^{\prime \prime} \times 76^{\circ}\right)$ cempered & 163 \\
\hline G1.28* & Solar glass $\left(46^{\prime \prime} \times 84^{7}\right)$ cempered & 100 \\
\hline GL32 & Solar glass $\left(46^{\prime \prime} \times 967\right)$ cempered & 165 \\
\hline GL40 & Solar glass $\left(46^{\prime \prime} \times 120^{\prime}\right)$ tempered & 304 \\
\hline GLC & Solar glass crave (per 1-3 shoets) & 58 \\
\hline
\end{tabular}

\begin{tabular}{|c|c|c|}
\hline \multicolumn{3}{|c|}{ Solar Collector Absorbers and Headers, (packaging not included) } \\
\hline$A G 25 *$ & $4^{\prime} \times 6.5^{\prime}$ grid, $3 / 4^{\circ}$ headers & 316 \\
\hline$A G 32-P$ & $4^{\prime} \times 8^{\prime}$ grid, $3 / 4^{\prime}$ henders, semi-selective painted surface & 330 \\
\hline$A G 32$ - SP & $4^{\prime} \times 8^{\prime}$ gric, $3 / 4^{\prime}$ headers, selective surface & 432 \\
\hline$A 640-P$ & I' $\times 10^{\prime}$ grid, $3 / 4^{\prime \prime}$ headers & 406 \\
\hline $\mathrm{A} 5255^{*}$ & $4 \times 6.5$ serpendine & 254 \\
\hline$A \leqslant 32-P$ & $4^{\prime} \times g$ sarpentine, semi-selective painted surface & 296 \\
\hline$A 532-5 P$ & $4 \times 8$ serpentinie, solective sturface & 400 \\
\hline$A S 40-P$ & $4^{\prime} \times 10^{\prime}$ serpentine & 393 \\
\hline RN12 & Round-rhombic ripple, $12 \mathrm{~mm}$ & 1.20 \\
\hline Ho75 & Header, 3/4", elw brazed nipples & 44 \\
\hline $\mathrm{H} 100$ & Header, $1^{4}$. clw brazed nipples & 61] \\
\hline
\end{tabular}

\begin{tabular}{|c|c|c|}
\hline \multicolumn{3}{|c|}{ Sunstrip $^{\text {Tu }}$ Solar Fin } \\
\hline$\$ S 146 / 12$ & $146 \mathrm{~mm}$ fin/12 $\mathrm{mm}$ ube (\$/sq $\mathrm{m})$ & 48 \\
\hline$\$ S 14 Q / 12-P$ & $146 \mathrm{~mm}$ fin/12 mm cube, semi-selective surface (\$/sq.m) & 57 \\
\hline$\$ S 146 / 12-S P$ & $146 \mathrm{~mm}$ fin/ $12 \mathrm{~mm}$ cube, selective surface (\$/sqm) & 92 \\
\hline SScutting: & Cucuing charge for in & 0,71 \\
\hline
\end{tabular}

\begin{tabular}{|c|c|c|}
\hline \multicolumn{3}{|c|}{ DTL Shell-and-tube Heat Exchangers } \\
\hline D2T7L3 & $2^{\prime}$ by $3^{\prime}$ all copper shell-and-rube & 458 \\
\hline & each extra foor add & 86 \\
\hline D3TIAL3 & $3^{\prime \prime}$ by $3^{\prime}$ all copper tube shell-and-tube & 727 \\
\hline & each excun foot add & 131 \\
\hline DAT28t.4 & $4^{\prime \prime}$ by $4^{\prime}$ all copper shell-and-tube. & 1472 \\
\hline & each extra foor add & 226 \\
\hline
\end{tabular}




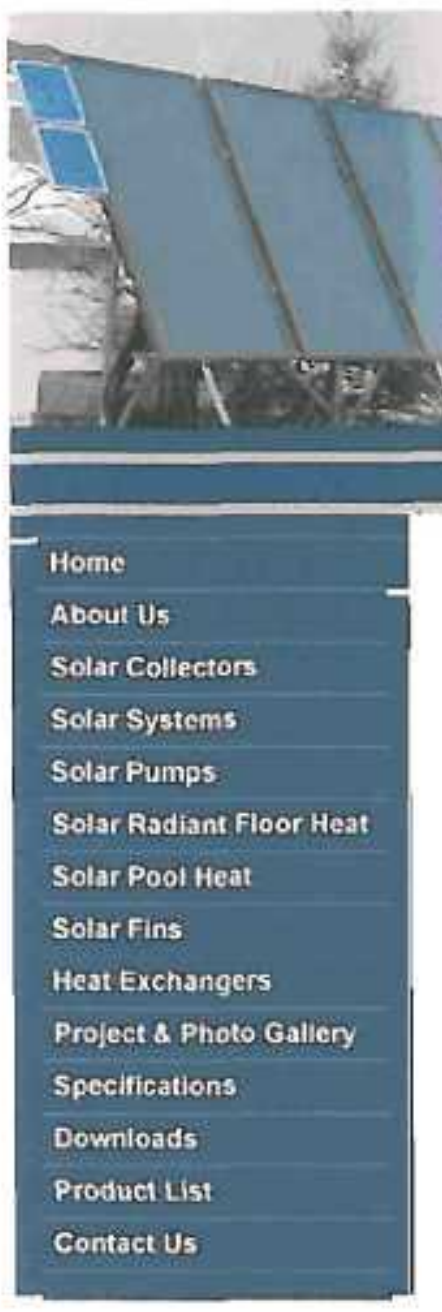

\title{
Thermo Dynamics Ltd. Solar Heating
}

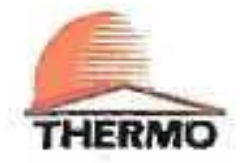

\section{SOLAR BOILER ${ }^{\mathrm{TM}}$ - Solar Water Heater}

\author{
Solar Domestic Water Heating System
}

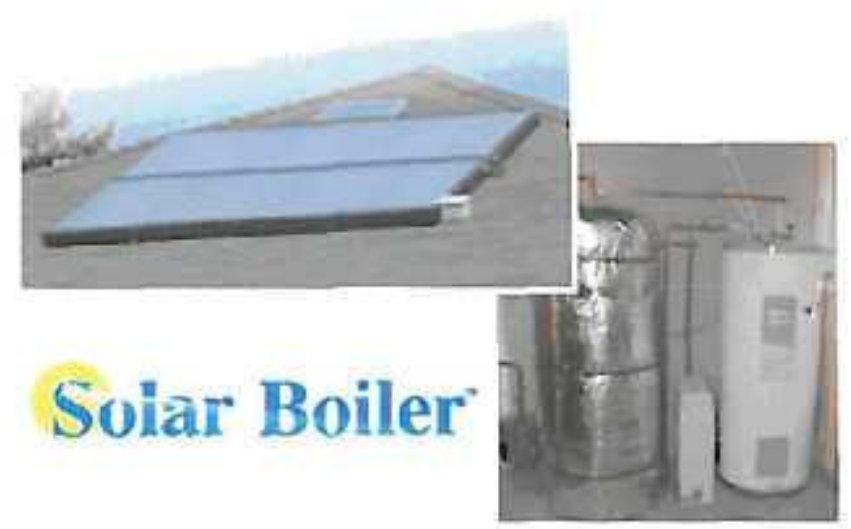

Environmentally-Friendly Technology That Can Save You Money.

The Solar Boiler collects energy from the sun and converts it into hot water.

Domestic hot water is the second-highest energy cost in the typical household. In fact, for some homes it can be the highest energy expenditure. Solar water heating can now reduce your domestic water heating costs by as much as $65 \%$.

The Thermo Dynamics' Solar Bolier is today's state-of-the-art solar water-heating appliance. Designed to pre-heat the domestic water that is supplied to your conventional water heater, it can result in remarkable savings. It's easy to instail and maintenance free. The instaliation package contains all the necessary hardware and complete instructions. As a homeowner, you can install it yourself or have your locai Thermo Dynamics dealer do it for you.

All parts of the system are built to demanding speciflcations, employing the latest solar technology for maximum performance. The Solar Boiler is manufactured from readily-available components, ensuring lower costs and reliability. The solar collectors are constructed of aluminum, copper, and tempered giass. The assembly incorporates the MICRO-FLO@ absorber, a continuous tube with only two connections for effortless installation. 


\section{Your investment in clean and natural.}

The Solar Boller is environmentally-friendiy. It does not pollute or use valuable nonrenewable resources. Even the pump to transport heat from the collectors to the storage tank is powered by the sun. Solar energy is a sound investment in everyone's future, today.

The Solat Boiler is designed for automatic daily and year round operation. A nontoxic glycol solution in the solar collectors ellminates winter freezing probiems. A photovoltaic module regulates the proper daily operation of the Solar Boiler and the solar loop circulator tums on only when the panels are hot enough to heat the solar tank.

As one of Thermo Dynamics' new generation of solar products, the Solar Boiler makes solar energy economically available to your family's needs. It's an investment: in reliable performance.

You have our name on it!

How it works:

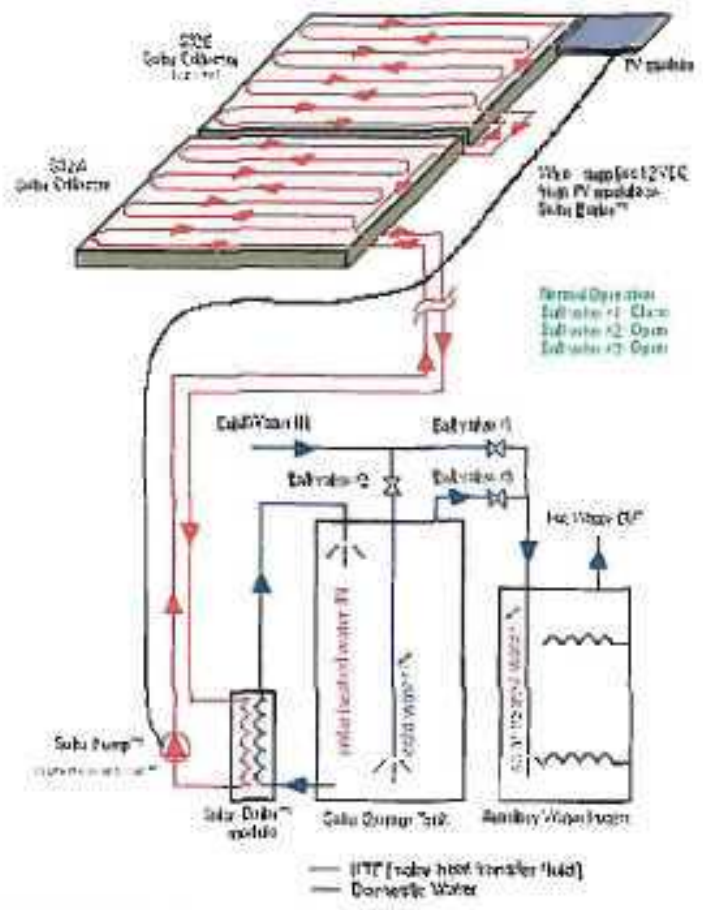

How the Soiar Boller the works

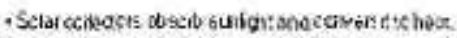

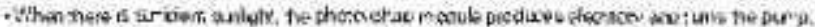

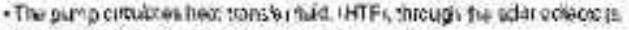

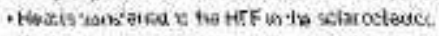

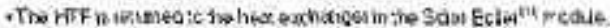

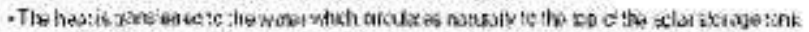

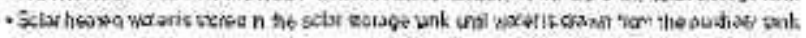

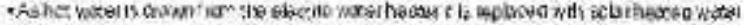

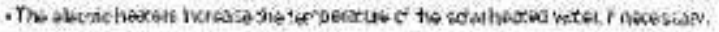

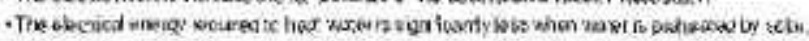

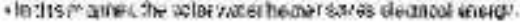


Light from the sun strikes the solar collector and heats the black metal Sunstrip absorber underneath the glass cover. This heat is transferred to a non-toxic antifreeze solution (propylene glycol and water) that is pumped through the collector and returns to the Solar Boller.

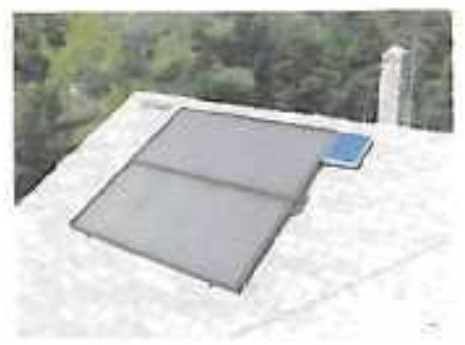

The hot glycol then transfers heat to the water in the storage tank via the Solar Boiler's heat exchanger. The water is heated repeatedly in this fashion, until the solar tank is hot.

The Solar Boiler is used as a domestic water pre-heater in conjunction with your conventional heating system. Cold water enters the Solar Boiler for initial heating, and is then delivered to the backup or conventional heating system for final heating as required. The conventional system is typically fueled by oil, electricity, propane, natural gas, etc....

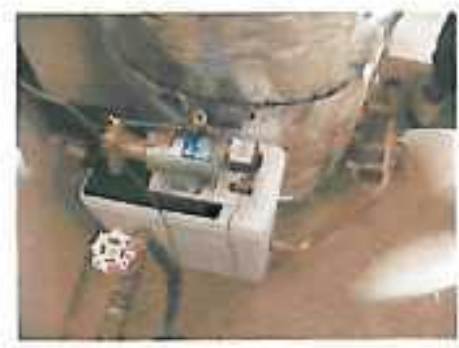

Conventional energy requirements can be reduced substantially by using the Solar Boiler, and on many days the Solar Boiler will provide ample hot water without the backup (conventional) heater turning on. In most families, the Solar Boiler will displace up to $65 \%$ of the water heating requirements. A typical system for a family of four would include two solar paneis ( 6 square meters), 270 litres of solar water storage (not included with the system), and a photovoltaic module to drive the glycol pump.

\section{Sizing Your Solar Domestic Hot Water System}

\begin{tabular}{|c|c|c|c|c|c|c|}
\hline $\begin{array}{l}\text { Family } \\
\text { Size }\end{array}$ & $\begin{array}{l}\text { System } \\
\text { Model }\end{array}$ & $\begin{array}{l}\text { Solar } \\
\text { Collectors }\end{array}$ & $\begin{array}{l}\text { Collector } \\
\text { Area }\end{array}$ & $\begin{array}{l}\text { Solar Dolier } \\
\text { Module }\end{array}$ & DHW Load & $\begin{array}{l}\text { Recommended } \\
\text { Solar Storage } \\
\text { Capacity* }\end{array}$ \\
\hline $\mid \begin{array}{l}1-3 \\
\text { people }\end{array}$ & SB32-9PV & $1-\$ 32 B$ & $\begin{array}{l}2.97 \mathrm{~m} 2 \\
(32 \mathrm{ft} 2)\end{array}$ & SBM-9DC & $\begin{array}{l}<250 \text { liters/day } \\
(<55 \text { IG/day }) \\
(<66 \text { USG/day) }\end{array}$ & $\begin{array}{l}273 \text { liters } \\
(60 \text { IG) } \\
(80 \text { USG) }\end{array}$ \\
\hline $\begin{array}{l}\text { - }-7 \\
\text { people }\end{array}$ & SB64-9PN & $\begin{array}{l}1-532 A \\
1-532 B\end{array}$ & $\begin{array}{l}5.82 \mathrm{~m} 2 \\
(64 \mathrm{ft2})\end{array}$ & SBM-90C & $\begin{array}{l}>250 \text { hters/day } \\
(>55 \mathrm{lG} / \mathrm{day}) \\
(>66 \mathrm{USG} / \mathrm{day})\end{array}$ & $\begin{array}{l}454 \text { liters } \\
(100 \text { IG) } \\
(120 \text { USG) }\end{array}$ \\
\hline
\end{tabular}

- Solar storage tanks are avaikable from Thermo Dynamics Lud. The solar storage tanks are not included with the system. Thermo Oynarnics Ltd. recommends the use of an electric water heater with the elements disconnected for a sofar storage tank. These can De obtained 


\section{System Components}

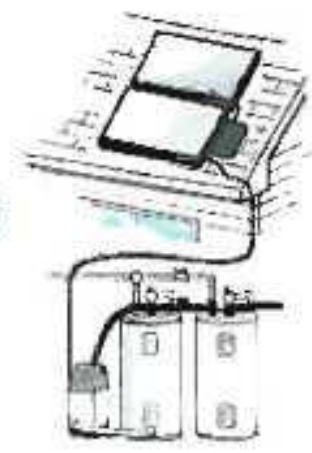

\section{SB32-9pV Solar Boiler System - $\$ 3,764.00$ CAD}

\begin{tabular}{|c|c|c|}
\hline Quantity & Model & Description \\
\hline 1 & $532 \mathrm{~B}-\mathrm{P}$ & $\begin{array}{l}4 \pi \times 8 \pi \text { Serpentine solar collector, "B", } 2 \text { connection ports + } \\
\text { sensor well }\end{array}$ \\
\hline 1 & SBM-90C & $\begin{array}{l}\text { Solar Bofler Module } \\
\text { - Solar Pump } \\
\text { - Expanslon tank } \\
\text { - Glycol Resevolr [complete with } 4 \text { liters of } \\
\text { glycol] } \\
\text { - Glycol inleVoutiet ports } \\
\text { - Water intet/outlet ports }\end{array}$ \\
\hline I & PV20 & 20 Wp photovoltalc module \\
\hline I & K1050 & Serpentine collector mounting kit Imounts flush to roof] \\
\hline 1 & K1060 & Pv mountieg $\mathrm{kt}$ \\
\hline 1 & $\times 2030-50$ & $\begin{array}{l}50 \text { Copper tube kit [comects collector to Solar Boller modale, } \\
\text { So r distance between] } \\
-50 \mathrm{ft} \text { supply copper tube, } 3 / 8^{*} \text { dia } \\
-50 \mathrm{ft} \text { retum copper tube, } 3 / \mathrm{B}^{\prime \prime} \text { dia } \\
-50 \mathrm{ft} 18 / 4 \mathrm{LVT} \text { wire } \\
-100 \mathrm{ft} \text { plpe insulation }\end{array}$ \\
\hline 1 & GLYUSM & 4 liter of Glycol for topping up the system \\
\hline
\end{tabular}

\section{SB64-9PV Solar Boiler System - \$4,659.00 CAD}

\begin{tabular}{|c|c|c|}
\hline Quantity & Model & Description \\
\hline 1 & $532 A \cdot \mathrm{P}$ & $4 \mathrm{ft} \times$ Bft: Serpentine solar collector, "A", 4 connection ports \\
\hline 1 & $\$ 328-P$ & $\begin{array}{l}4 \mathrm{Af} \times \text { 8ft Serpentine } 50 \text { lar collector, "B", } 2 \text { connection ports }+ \\
\text { sensor well }\end{array}$ \\
\hline 1 & SBM-9DC & \begin{tabular}{|l} 
Solar Boller Module \\
- Solar Pump \\
- Expansion tank \\
- Glycol Resevoir [compiete with 4 liters of \\
gtycol] \\
- Glycol Inlet/outlet ports \\
- Water inlet/outlet ports \\
\end{tabular} \\
\hline 1 & PV20 & 20 Wp photovoltatic module \\
\hline 1 & 1.055 & Serperitine collector mounting kit [mounts flush to roof] \\
\hline 1 & $K 1060$ & PV mounting kit \\
\hline
\end{tabular}




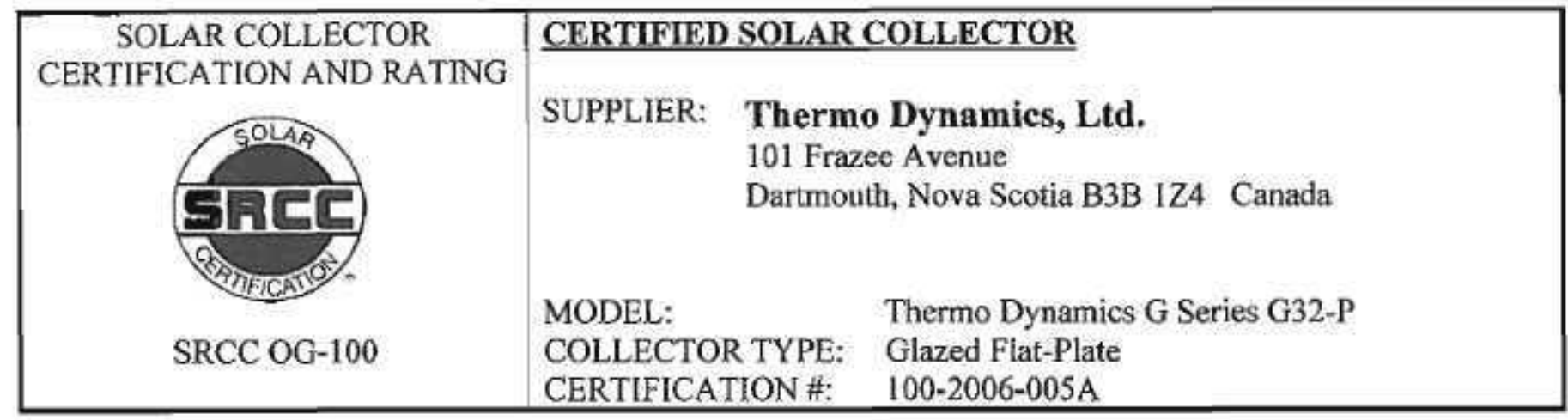

\begin{tabular}{|c|c|c|c|c|c|c|c|}
\hline \multicolumn{8}{|c|}{ COLLECTOR THERMAL PERFORMANCE RATING } \\
\hline \multicolumn{4}{|c|}{ Megajoules Per Panel Per Day } & \multicolumn{4}{|c|}{ Thousands of Btu Per Panel Per Day } \\
\hline $\begin{array}{c}\text { CATEGORY } \\
\text { (Ti-Ta) }\end{array}$ & $\begin{array}{l}\text { CLEAR } \\
\text { DAY } \\
23 \mathrm{MJ} / \mathrm{m}^{2} \cdot \mathrm{d} \\
6.39\end{array}$ & $\begin{array}{l}\text { MILDLY } \\
\text { CLOUDY } \\
17 \mathrm{MJ} / \mathrm{m}^{2} \cdot \mathrm{d} \\
4.73^{2}\end{array}$ & $\begin{array}{l}\text { CLOUDY } \\
\text { DAY } \\
11 \mathrm{MJ} / \mathrm{m}^{2} \cdot \mathrm{d}\end{array}$ & $\begin{array}{c}\text { CATEGORY } \\
(\mathrm{T} \mathrm{i}-\mathrm{Ta})\end{array}$ & $\begin{array}{c}\text { CLEAR } \\
\text { DAY } \\
2000 \\
\text { Btw/ft'd }\end{array}$ & $\begin{array}{c}\text { MILDLY } \\
\text { CLOUDY } \\
1500 \mathrm{Btw} / \mathrm{ft}^{2}-\mathrm{d}\end{array}$ & $\begin{array}{l}\text { CLOUDY } \\
\text { DAY } \\
1000 \mathrm{Btw} / \mathrm{At}^{2}-\mathrm{d}\end{array}$ \\
\hline A $\quad\left(-5^{\circ} \mathrm{C}\right)$ & 42 & 32 & 21 & A $\quad\left(-9^{\circ} \mathrm{F}\right)$ & 40 & 30 & 20 \\
\hline B $\quad\left(5^{\circ} \mathrm{C}\right)$ & 37 & 27 & 17 & $\left(9^{\circ} \mathrm{F}\right)$ & 35 & 26 & 16 \\
\hline C $\left(20^{\circ} \mathrm{C}\right)$ & 30 & 20 & 10 & C $\quad\left(36^{\circ} \mathrm{F}\right)$ & 29 & 19 & 10 \\
\hline D $\left(50^{\circ} \mathrm{C}\right)$ & 17 & 8 & 1 & D $\left(90^{\circ} \mathrm{F}\right)$ & 16 & 8 & 1 \\
\hline E $\left(80^{\circ} \mathrm{C}\right)$ & 6 & & & E $\left(144^{\circ} \mathrm{F}\right)$ & 5 & & \\
\hline
\end{tabular}

A-Pool Heating (Warm Climate) B-Pcol Heating (Cool Climate) C-Water Heating (Warm Climate) D-Water Heating (Cool Climate) E-Air Conditioning

Original Certification Date: March 12, 2007

COLLECTOR SPECIFICATIONS

$\begin{array}{lrlrllrlrl}\text { Gross Area: } & 2.982 & \mathrm{~m}^{2} & 32.10 & \mathrm{ft}^{2} & \text { Net Aperture Area: } & 2.783 & \mathrm{~m}^{2} & 29.96 & \mathrm{ft}^{2} \\ \text { Dry Weight: } & 43.5 & \mathrm{~kg} & 96 & \mathrm{lb} & \text { Fluid Capacity: } & 2.3 & \mathrm{I} & 0.6 & \mathrm{gal} \\ \text { Test Pressure: } & 1103 & \mathrm{kPa} & 160 & \mathrm{psig} & & & & & \end{array}$

\section{COLLECTOR MATERIALS}

$\begin{array}{ll}\text { Frame: } & \text { Aluminum } \\ \text { Cover (Onter): } & \text { Low Iron Tempered Glass } \\ \text { Cover (Inner): } & \text { None } \\ \text { Absorber Material: } & \text { Tube-Copper / Plate - Aluminum } \\ \text { Absorber Coating: } & \text { Moderately Selective Black Paint } \\ \text { Insulation (Side): } & \text { Fiberglass } \\ \text { Insulation (Back): } & \text { Fiberglass }\end{array}$

PRESSURE DROP

\begin{tabular}{|c|c|c|c|}
\hline \multicolumn{2}{|c|}{ Flow } & \multicolumn{2}{c|}{$\Delta \mathbf{P}$} \\
\hline $\mathrm{mI} / \mathrm{s}$ & gpm & $\mathbf{P a}$ & in $\mathbf{H}_{\mathbf{2}} \mathbf{O}$ \\
\hline 20 & 0.32 & 73 & 0.29 \\
50 & 0.79 & 228 & 0.91 \\
80 & 1.27 & 437 & 1.75 \\
\hline
\end{tabular}

TECHNICAL INFORMATION

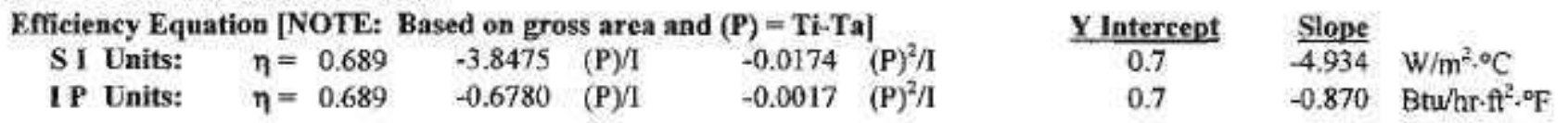

Incident Angle Modifier $\left[(\mathrm{S})=1 / \cos \theta-1,0^{\circ} \leq \theta \leq 60^{\circ}\right]$
$K_{\mathrm{ar}}=1,0$
(S)
$\mathbf{K}_{\mathrm{ar}}=1.0$
$-0.4920$
(S)
$-0.1291(\mathrm{~S})^{2}$
(Linear Fit)

Model Tested:

G32-P

Test Fluid: Water

Test Flow Rate: $\quad 60 \mathrm{~m} / \mathrm{s} \quad 0.94 \quad \mathrm{gpm}$

\section{REMARKS:}

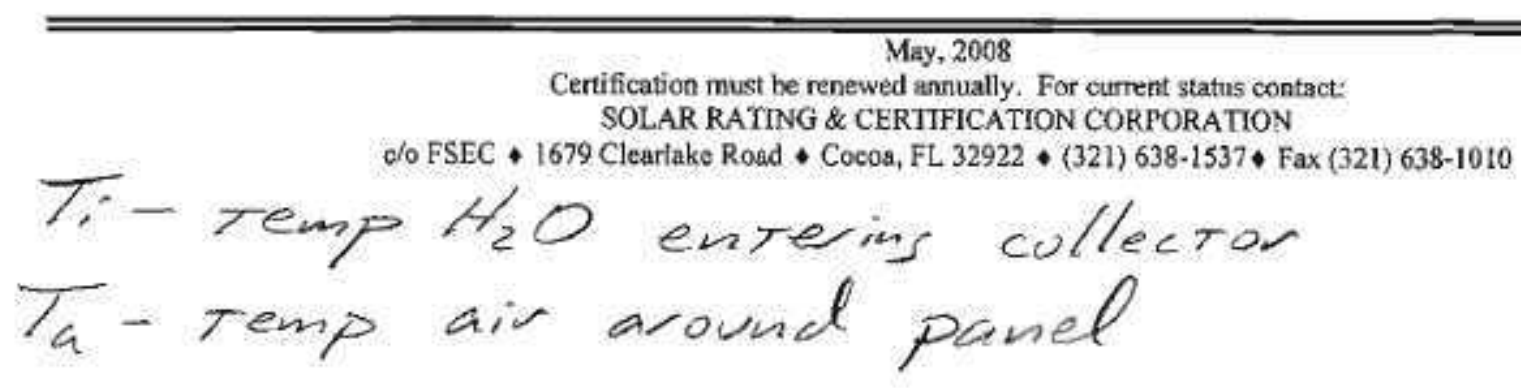




\section{Evergreen Solar UT 2500 EG

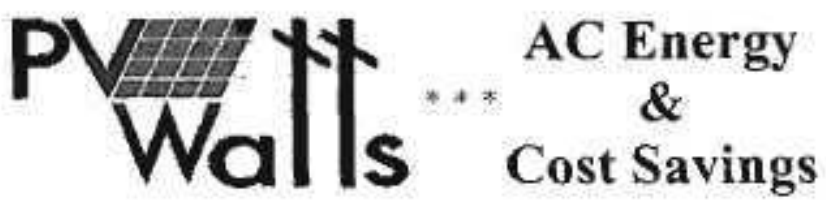

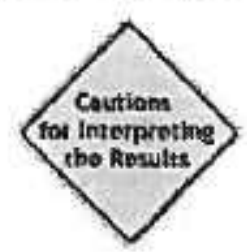

\begin{tabular}{|l||l||}
\hline \multicolumn{2}{|c|}{ Station Identification } \\
\hline \hline City: & Flint \\
\hline \hline State: & MI \\
\hline \hline Latitude: & $42.97^{\circ} \mathrm{N}$ \\
\hline \hline Longitude: & $83.73^{\circ} \mathrm{W}$ \\
\hline Elevation: & $233 \mathrm{~m}$ \\
\hline PV System Specifications \\
\hline \hline DC Rating: & $3.0 \mathrm{~kW}$ \\
\hline \hline DC to AC Derate Factor: & 0.770 \\
\hline \hline AC Rating: & $2.3 \mathrm{~kW}$ \\
\hline \hline Array Type: & Fixed Tilt \\
\hline \hline Array Tilt: & $43.0^{\circ}$ \\
\hline \hline Array Azimuth: & $180.0^{\circ}$ \\
\hline \hline Energy Specifications \\
\hline Cost of Electricity: \\
\hline \hline
\end{tabular}

Output Hourly Performance Data

About the Hourly Performance Data

\begin{tabular}{|c|c|c|c|}
\hline \multicolumn{4}{|c|}{ Results } \\
\hline Month & $\begin{array}{c}\text { Solar } \\
\text { Radiation } \\
\left(\mathrm{kWh} / \mathrm{m}^{2} / \text { day }\right)\end{array}$ & $\begin{array}{c}\text { AC } \\
\text { Energy } \\
(\mathrm{kWh})\end{array}$ & $\begin{array}{c}\text { Energy } \\
\text { Value } \\
\text { (\$) }\end{array}$ \\
\hline 1 & 2.69 & 201 & 16.68 \\
\hline 2 & 3.79 & 255 & 21.16 \\
\hline 3 & 4.30 & 309 & 25,65 \\
\hline 4 & 4.99 & 335 & 27.80 \\
\hline 5 & 5.43 & 365 & 30.30 \\
\hline 6 & 5.57 & 353 & 29.30 \\
\hline 7 & 5.43 & 345 & 28.64 \\
\hline 8 & 5.34 & 347 & 28.80 \\
\hline 9 & 4.77 & 307 & 25.48 \\
\hline 10 & 3.83 & 259 & 21.50 \\
\hline 11 & 2.41 & 162 & 13.45 \\
\hline 12 & 1.79 & 127 & 10.54 \\
\hline Year & 4.19 & 3365 & 279.30 \\
\hline
\end{tabular}

Output Results as Text

*

Saving Text from a Browset

Run PVWATTS $\times .1$ for another US location or an International location

Run PVWATTS v.2 (US only)

Please send questions and comments regarding PWWATTS to Webmaster

Disclaimer and copyright notice

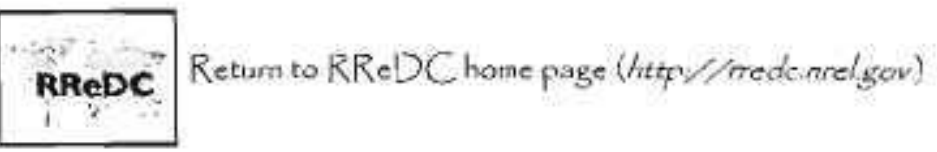




\section{$\underset{\text { evergreensolar }}{\text { Think Beyond. }}$}

SPRUCE LINE ${ }^{\text {TM }}$

photovoltaic modules

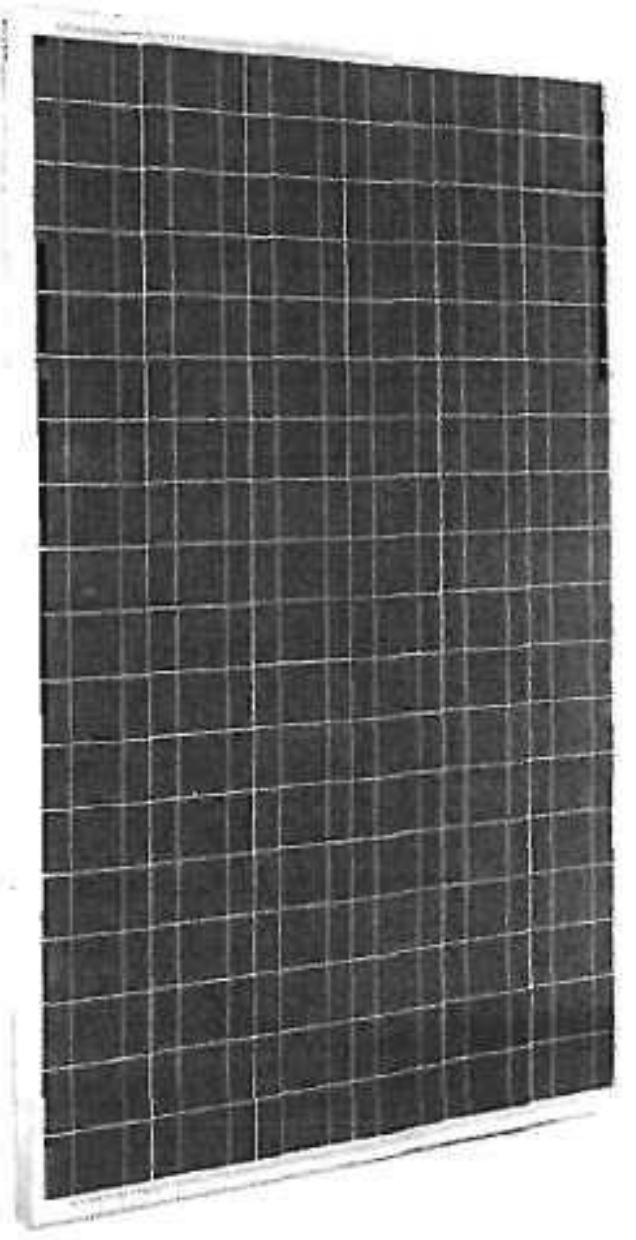

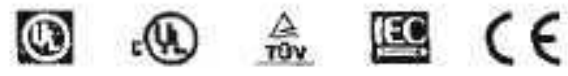

\section{W module}

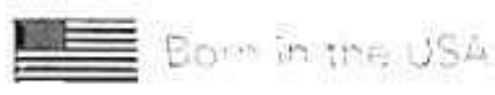

A high quality polycrystalline solar panel for on-grid markets offering exceptional performance, extraordinary versatility and industry-leading environmental credentials based on our cuttingedge String Ribbon" wafer technology.

- Superior performsnce under real field conditions

- Get up to $102.5 \%$ of rated power, with a guaranteed minimum of $98 \%$

- 5 year workmanship and 25 year power warranty for ultimate peace of mind

- More installation versatility with our extensive range of mounting options

- Higher strength with wind speeds guaranteed up to $130 \mathrm{mph}$ and snow loads up to $80 \mathrm{lbs} / \mathrm{ft}^{2}$

- Qualified to all major industry certifications and regulatory stanclards.

- Smallest carbon foot-print leading the fight against global warming

- Quickest energy payback time for the maximum energy conservation

- Cardboard-free packaging for minimal on-site waste and disposal cost

- Industry-first paid return and re-use program for product packaging

*For ful details soo the Evergreen Solar Umined Warranty available on request or online. 


\section{Electrical Characteristics}

Standard Test Conditions (STC)

\begin{tabular}{|c|c|c|}
\hline & & 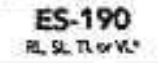 \\
\hline$P_{m \varphi^{2}}$ & (W) & 190 \\
\hline Protentice & (\%) & $-2 \% /+2.7 \%$ \\
\hline$P_{\text {ma, max }}$ & (W) & 194.9 \\
\hline$P_{m p, \operatorname{man}}$ & (W) & 186.2 \\
\hline$P_{p s}{ }^{3}$ & (W) & 168.8 \\
\hline$V_{m p}$ & $M$ & 26.7 \\
\hline$I_{m p}$ & (A) & 7.12 \\
\hline$V_{o r}$ & (N) & 32.8 \\
\hline los & (A) & 8.05 \\
\hline
\end{tabular}

Nominal Operating Cell

Temperature Conditions (NOCT)

\begin{tabular}{|lll|}
\hline$P_{m p}$ & $(W)$ & 136.7 \\
\hline$V_{m p}$ & $(N)$ & 23.8 \\
\hline$I_{m p}$ & $(A)$ & 5.75 \\
\hline$V_{o c}$ & $(N)$ & 30.3 \\
\hline$I_{\alpha}$ & $(A)$ & 6.46 \\
\hline$T_{\text {moct }}$ & $\left({ }^{\circ} \mathrm{C}\right)$ & 45.9 \\
\hline
\end{tabular}

$1000 \mathrm{~W} / \mathrm{m}^{2}, 25^{\circ} \mathrm{C}$ cell tomperaturs. AMK 1,5 spectrum;

'Mowimum power point or rated poner

tAt PV-USA Test Condtions: 1000 W/m, $200 \mathrm{C}$ mbient

berrovesturs, 1 m/s wind speec

$600 \mathrm{~W} / \mathrm{m}$ '. $200 \mathrm{C}$ ambient tempenture, $1 \mathrm{~m} / \mathrm{s}$ and speed. AMt is spectrum

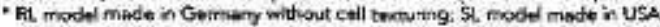

writhourt collt texwiringe $T$. model made in Germeny with sell bexturing. VI.

model made in U5A with cell taxhuring

Low Irradiance

The typical relative reduction of module efficiency at an irrediance of $200 \mathrm{~W} / \mathrm{m}^{2}$ in relation to $1000 \mathrm{~W} / \mathrm{m}^{2}$ both at $25^{\circ} \mathrm{C}$ cell ternperature and spectrum AM 1.5 is $0 \%$.

Temperature Coefficients

\begin{tabular}{|lll|}
\hline$a P_{m p}$ & $\left(\% /{ }^{\circ} \mathrm{C}\right)$ & -0.49 \\
\hline$a V_{m p}$ & $\left(\% /{ }^{\circ} \mathrm{C}\right)$ & -0.47 \\
\hline$a l_{m p}$ & $\left(\% /{ }^{\circ} \mathrm{C}\right)$ & -0.02 \\
\hline$\alpha V_{\omega}$ & $\left(\% /{ }^{\circ} \mathrm{C}\right)$ & -0.34 \\
\hline$\alpha l_{\alpha}$ & $\left(\% /{ }^{\circ} \mathrm{C}\right)$ & 0.06 \\
\hline
\end{tabular}

System Design

\begin{tabular}{|lc|}
\hline Series Fuse Rating & 15 A \\
\hline UL Rated Systern Voltage & $600 \mathrm{~V}$ \\
\hline
\end{tabular}

ELECTRICAL EQVAPMENT

CHECX WITH YOUR INSTALER

\section{Mechanical Specifications}

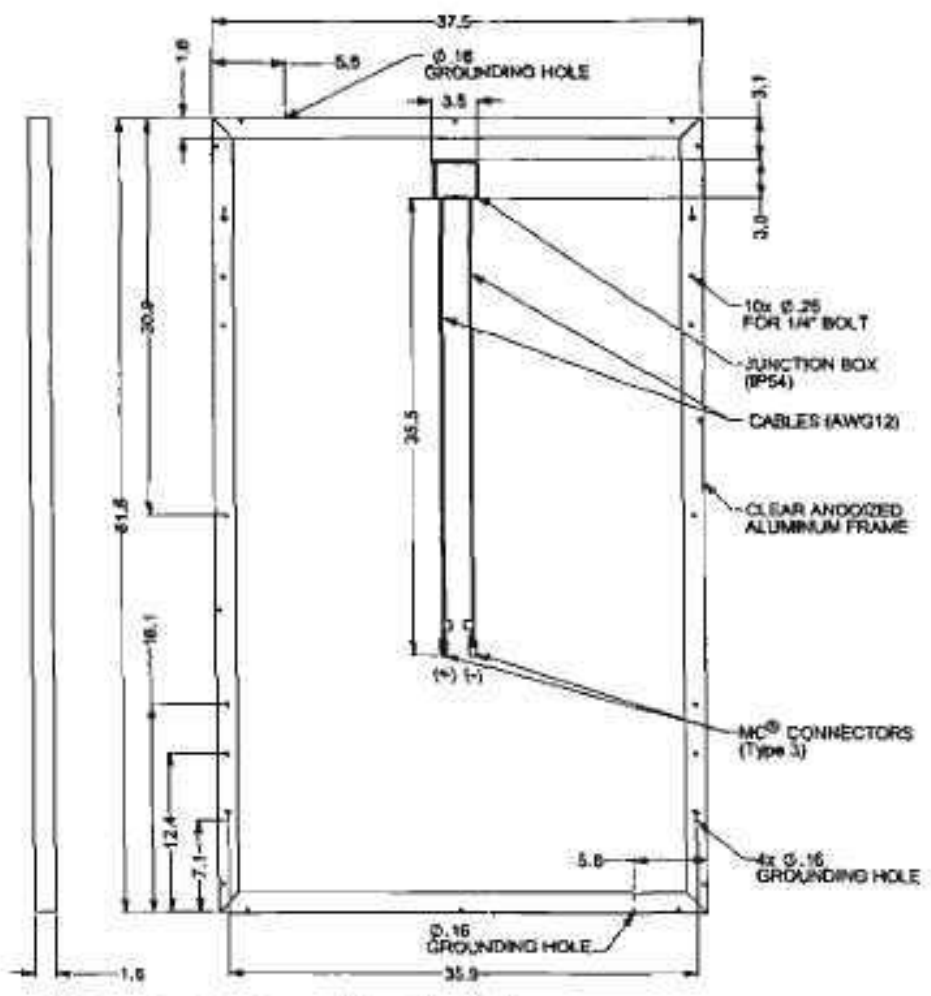

Afl dimensions in inches; module weight $40.1 \mathrm{lbs}$

Product coristructed with 108 polycrystelline silicon solar cells, anti-reflective tompered solat glass, EVA encepswlant, Tedlar beck-skin and a double-walled anodzed aluminum firame. Product packaging tested to Intemational Safe Transit Association OSTAN Standard 2E. All apecifications in this product information sheet conform to ENSOSEO. Sae the Evergreen Solar Salevy, instaliabon and Operation Manual and Mounting Design Guide for further informetion on approved installation and use of this product

Due to continuous innovation, reseirch and product improvement, the specifications in this product information sheet are subjoct to change without notice. No rights can be derived from this produet information sheet and Evergreen Solar assumen no liability whatsoever corrected to of resulting fiom the use of any information contained herein.

Partner:

\$190__US_010707; effective July 1st 2007 zvergreen Solar ine. www,evergreensolar.com
Worichwide Headquarters

138 Bartlett Street, Marlboro, MA 01752 USA

$T:+1508.357 .2221 \quad F:+1508.229 .0747$

infoecvergreensolar.com
Customar Service - Americas and Asla 138 Bartlett Street, Marlboro, MA 01752 USA I: $+1508.357 .2221 \quad F:+1508.229 .0747$ salesoovergreensolar.com 


\section{SOL-GEN ${ }^{\mathrm{m} M}$ UT Solar Electric Generators $\mathrm{N}$ vergreen Modules and Various Inverters}

These Systems put a reverse spin on your electric meter, and they qualify for a rebate from the California Public Utilities Commission and a $30 \%$ Federal Tax Credit (\$2000 max for residential)

SOL-GEN ${ }^{\text {ne }}$ UT systems automatically convert sunshine directly into electricity.

These systems produce clean, cost-effective power, silently, with no moving parts. When the power you generate is greater than what your house is using, your electric meter will run backwards and reduce your utility bill. Current laws require the utility companies to credit you for the power at the same rate you pay up to break even. These systems include a choice of several high quality inverters and all the major components needed for a complete instailation.

The modules are Evergreen Spruce Line String Ribbon 190 watt. These modules come with a $98 \%$ guaranteed power output (2\% power tolerance) and a 25 year power output warranty. The flush mounting hardware included is fabricated with stainless steel hardware and anodized aluminum, engineered and designed for rapid, durable installation. All switchgear and ground fault protection for the array are Included and UL listed. An approved system performance meter is included. It shows the instantaneous power output, as well as dally and cumulative KWH production. In the event of a utility power failure these systems will not operate. If you would like a system that would protect you from blackouts, select one of our SOL-GEN'M UB systems. Feeding power back to the utility grid requires approval from your utility company. Similar systems available for 3-phase $208 \mathrm{VAC}$ output. Installation cost will vary depending on the size of the system, type of roof and location. System ratings below are not adjusted for geographic or design factors. These factors may reduce the system rating based on site specific factors.

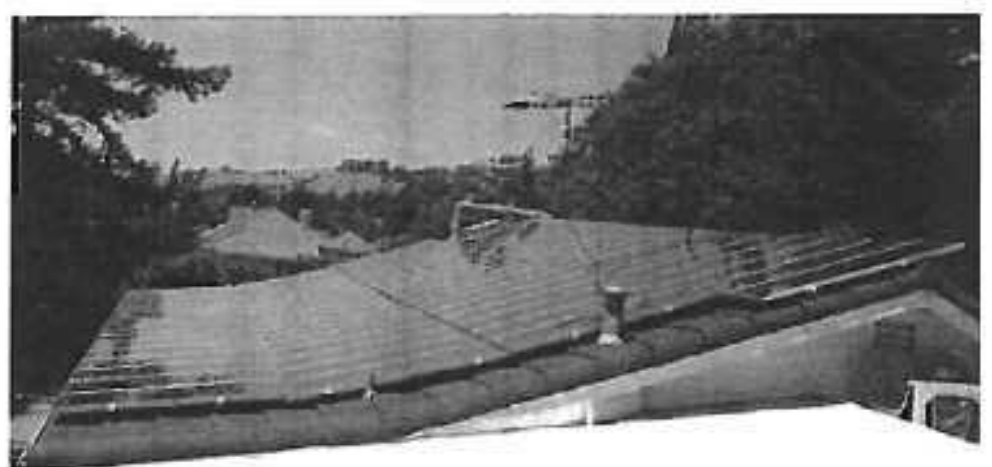

Sol-Gen UT Systems w/ Evergreen 190 watt Modules and Various Inverters

\begin{tabular}{|c|c|c|c|c|c|c|c|c|}
\hline Model Number & UT2500EG & UT3200EF & UT3800EF & UT4500EP & UT4800ES & UT4800EG & UT5500ES & UT6800ES \\
\hline Order Number & 101032 & 105872 & 105881 & 105882 & 104384 & 102322 & 105883 & 101038 \\
\hline PVPeak Power (Watts AC) CEC & 2539 & 3173 & 3808 & 4537 & 4836 & 4836 & 5510 & 6806 \\
\hline No of Strings, No. per String & $1 \times 18$ & $2 \times 10$ & $2 \times 12$ & $2 \times 14$ & $2 \times 15$ & $2 \times 15$ & $2 \times 17$ & $3 \times 14$ \\
\hline PV Avray Area Sg. Ft. (Approx) & 262 & 332 & 400 & 462 & 495 & 495 & 560 & 680 \\
\hline Average KWH/Day (CEC) & 12.7 & 15.9 & 19.0 & 22.7 & 24.2 & 24.2 & 27.6 & 34.0 \\
\hline Inverter Model & Gr 2800 & 164000 & IG4000 & PVP 4800 & SMA 5000US & GT5000 & SMA600OUS & SMA7000US \\
\hline ListPrice & $\$ 22,161$ & $\$ 28,573$ & $\$ 33,529$ & $\$ 38,381$ & $\$ 41,119$ & $\$ 40,743$ & $\$ 46,464$ & $\$ 56,558$ \\
\hline PGEE / CCSE Rebate (@\$2.20Matt) & $\$ 5,586$ & $\$ 6,981$ & $\$ 8,378$ & $\$ 9,981$ & $\$ 10,639$ & $\$ 10,639$ & $\$ 12,122$ & $\$ 14,973$ \\
\hline SCE Rebate (@\$2.50Matt) & $\$ 6,348$ & $\$ 7.933$ & $\$ 9,520$ & $\$ 11,343$ & $\$ 12,090$ & $\$ 12,090$ & $\$ 13,775$ & $\$ 17,016$ \\
\hline
\end{tabular}
$L=$ good for minimum Temp of 30 deg F or higher

Suggested retail prices do not include tax, ins tallation of permitting costs. Contractor discounts available.

-Estimate based on 5 ESH Equivalent Sun House, south facing array, Design \& Geographic Factor of 1.0. May be lower based on site specific factors. Rev 1 -08

$$
\text { C3040 W Peak DC }
$$


Appendix D. Small Wood Boiler Spec Sheet 


\section{BIOHEAT -}

\section{$\frac{\dot{0} 00}{Q}$}




\section{jolo Plus}

Solo Plus Applications

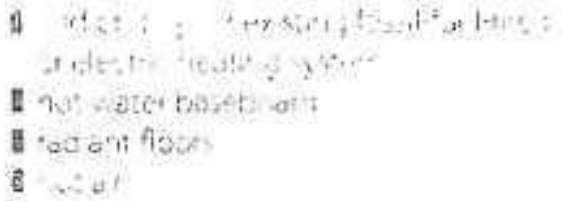

\section{Wood-fired Gasification Add-on Boiler}

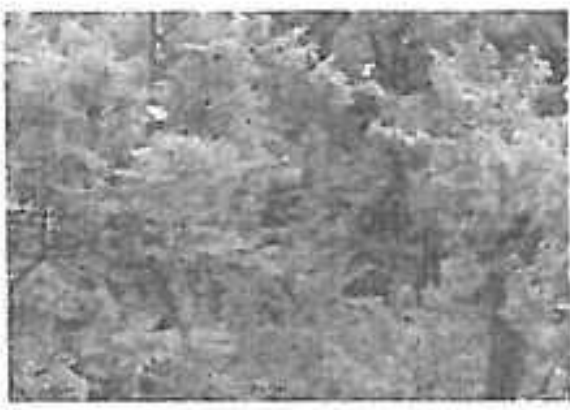

Independence and Self-Reliance soo $0^{\circ}$ us boiters ornvide a convenient. sale and enviomentisaly resporisible way ic hea? wa r home and hot water w.tn waod Solo Jils owriers areersured

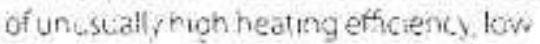
rearinc cost and use of en abundart. locally avalable renewable fuel the SoP LS wuodgasticatim combustion

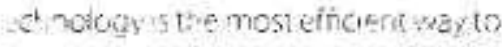
Dym torkwood As a resut ine $500^{\circ}$ us bole ises substanif aliy less wood han cotverito-s wood to exs diciolidocor watel stoves Aadironaily this high. efficienc) buln:erchologr proouces

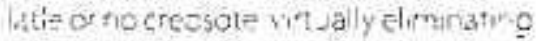
the tis of chmney fres and greatly reduc ng gieen nouse gas enusso is.

\section{Sizing Your Boiler}

As whitiany heatme system, choosing in aboronrutofy szed reatsouces fiet essary to opamize efficitency ease ol Extratich and hume comforl Be waty of using simiple techniques tased onty on square footage The heat oic of a nome can vas) wicel, dependuc on ag=and sype of consinuction, type of heating

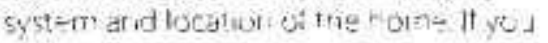
riave questions abo, ll with boler is stos: approprate ior yout reeds please coniser yout locdi bioteatus A deale of contact usdtrecty at sur toll tree number ind we vrould be -appy so duscuiss you acpicetion with you

\section{Reliability}

The intermal oatal is made of fully weided $7 \mathrm{~mm}$ thick p.ate stee 310 -ieat USA polem are conseructed to furopedr

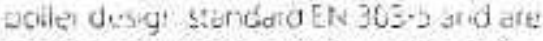
cesonet ot soless.rized sysims Solo Plus ticilers are $U$ and UlC aproved.

\section{Efficiency}

The Soig Pius boler uses obind dra's casficationiectindony to achieve very tighefficioncy thekevic this process is the high temperature 1300 or more, redthed ir a secand refractory-imed rembustion chambet This secondarycomiustion corisumes the creosote ancisrnone that formally goes up ine chimney, inereby winging every but of energy ow of the wooc fuel and resuling nawry clean bur?

\section{About BioHeatUSA}

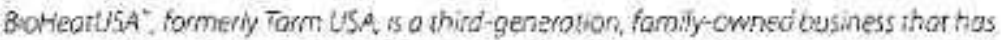
pianeerad the sales ond service of Europeon fesidentikf central heoting equipmenr in North America for over 30 years Biaheot USAS primary objective is to offer innovorhe home heating soiuticns, olong with o significant commitmient to consurner educotion and environmentof

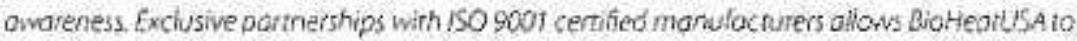
offer pioducls with cperabonatretiabsity, unique firng efficiency, and to promare the clech buming of cartwon-cycle biomass that is criticol to the fowering of ner greenthouse gos emissions

\section{About Scanditec}

Scandrec, locored in Shjern, Denmark, is a producer of centiol heoung biomass bollers, Fans Sorenson, the farmer Manoging Directur of Boxi, land granoson of the founder of HS Tarm). now owns and manages the compuny which employs on experienced teom of n\&D and production/distribution professionals.

The chree-inchvertica heatex harige whes are sized for maximum extract on of the enesgy in the higr-temperature exhaust strean Descherne verypigh seconcary sum ternperat Lies, stack temperatures :emain rolatively low Period c boushing of tre neatexchange. tubes wil heif maintaribuk-1 eficiency. ond is easily accomplished vathe hath on the rop of the bole?

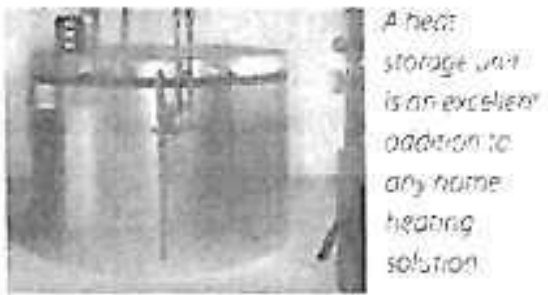

Optimization and Heat Storage Firevicoos burns most eficients and deanly wrien it is burned hot and test. Down otait gasfication faci tates jut this kisd of burn. Your home, however, doe's noi use haat in tas same way, it cails fot heai only as rifeded in arder to maten both g' these demands we recommiendi combring a vood bolier witn a water storage tanik of $500-1000$ ga ons. Insteao of smot ng and smowerng nide mode when your nome is up io temperature. a storage tank will allow the soic Plus to cominue to burn at maxmur efficiencs the excess near generates will smpiy be stored in the wates tank for aier jie A certan amount of iding is arceptede, and if yak are cnly insending to bur wood dung it e coldest warl or the year, no slorage tant is necessaty However, once the warm days of early soing arrive, it will be time to shut cown your woud boiler for that surrmer Ry incorfoctating thermat storage you maximize the fficiency ot your woco boler ard are able to use it througroul the spming and fall-and even light though 5trmer - io ptoduce vout domestic hal watet if you choose Therral siorage can easly be atdjed to a system al a ato date 


\section{Features}

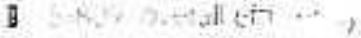

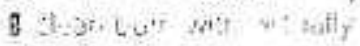

wosicritarcreonte

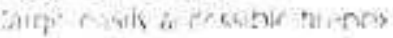

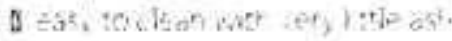

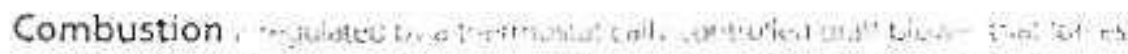

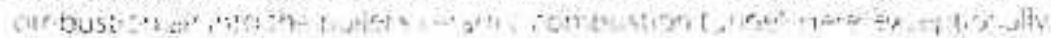

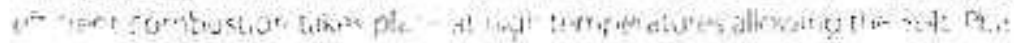

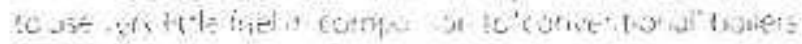

\section{Howltworks}

Solo Plus is a patented, woud-fired gasfication boiler available in three sizes with outputs from 100,000 to 199000 BTU/hr. The wood gasification combustion process within rhe Sclo Plus begins when the smail, quiet draft fan turns on in response to your home's thermostat.
The draft fan forces fresh air into the top of the frebox and down through the wood and live charcoat bed. This hot air and smoke stixture is forced through a slot in the top of the ceramic combustion chamber. Super-heated secondary air is injected into these gases. The correct combination of wood, gas, smoke and

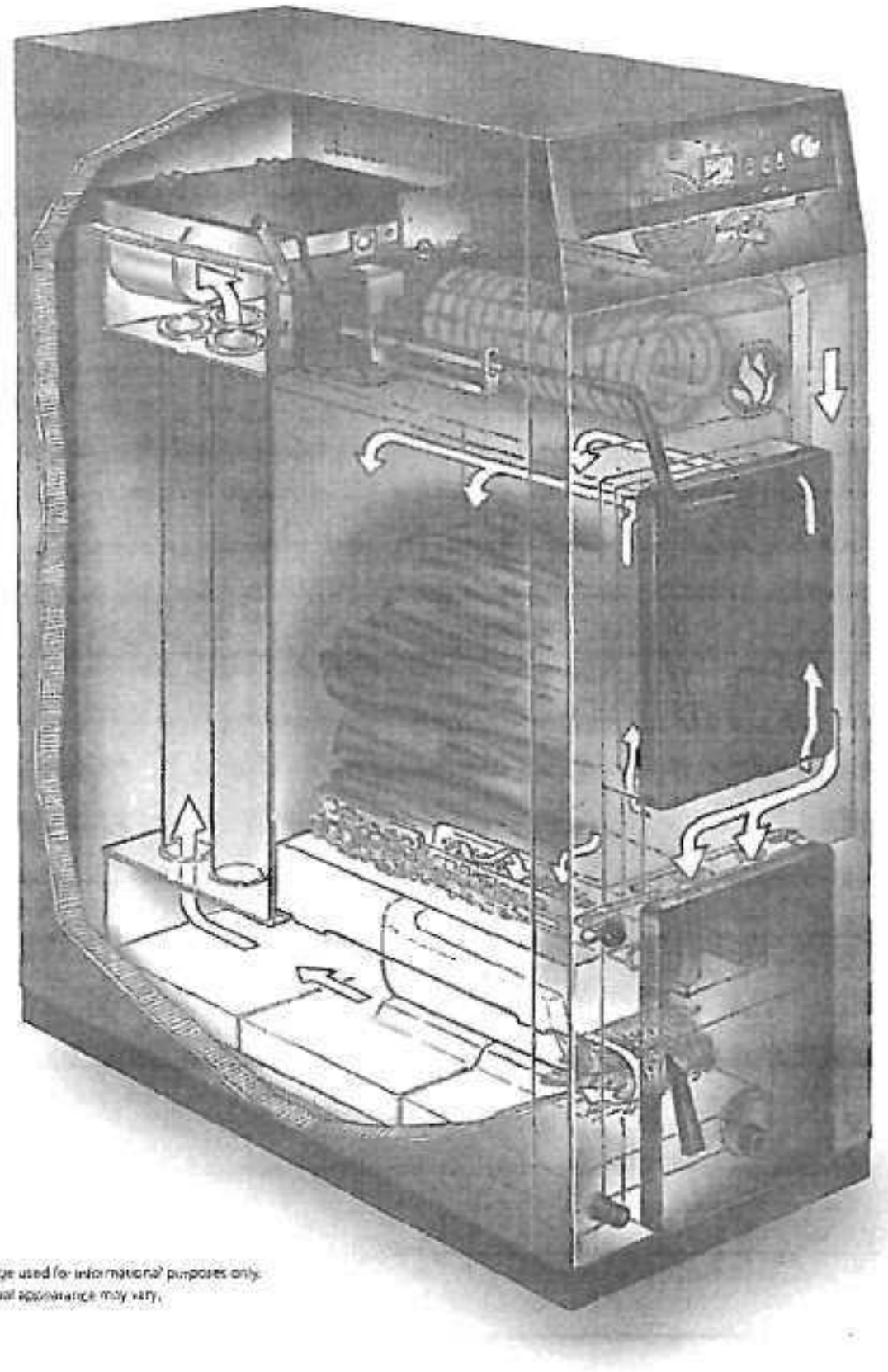

high-temperature oxygen resultsinan $3800^{\circ}$ flame in the ceramic combustion tunnel Gases stay in this hot, tutbulent enviorment long enough to achieve extremely high combustion efficiency. The resultanc high-temperature gases pass inta the vertical tieat exchange tubes, giving off heat to the boiler water for house heating and hot water demands.

\author{
Standard Equipment \\ 5ij jacket with insuletion \\ II steel doors with gasketing. \\ Ii draft fan \\ 1 religt valve \\ 11 boiler control \\ v cleaning implements \\ If manual
}

Optional Equipment

1 heat sorage system

11 domestic hot water coil

II Termovar botier protection valve

I contac Bionieat USA

for a fult ist of accessories

\section{Warranty}

Fach Solo Plus boiler is covered by a 20-year limited wartanty. A copy is avaliable for your inspection, and is provided with each boiler.

\section{Disclaimer}

BioHear USA is not responsible for factory aizerations to measurements. For final specifications, please see the Scandtec Solo Pius Owner's Manual. 


\section{3|ⒽEAT \\ 3.

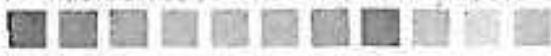
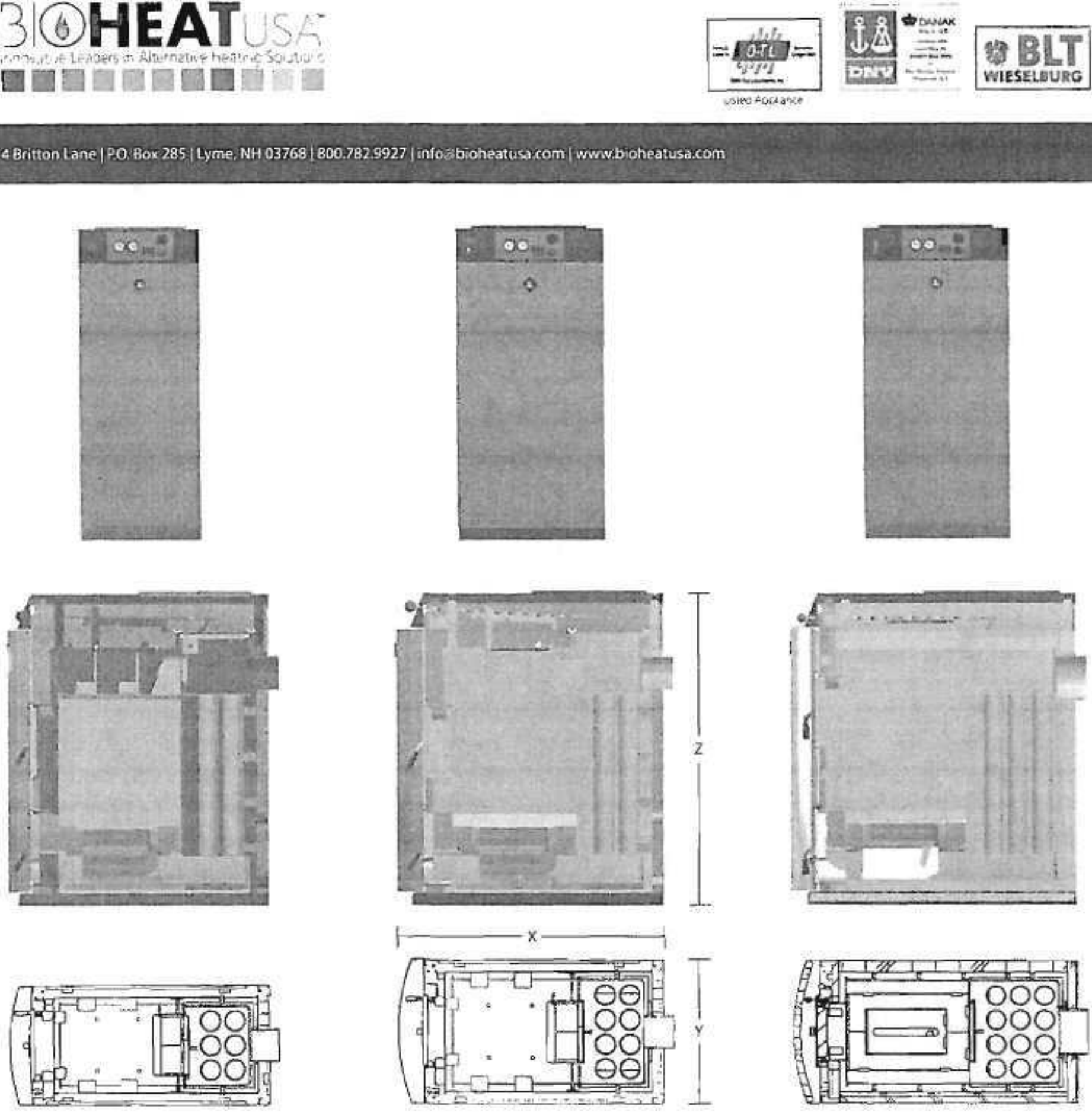

Solo Plus 30

Solo plus 40

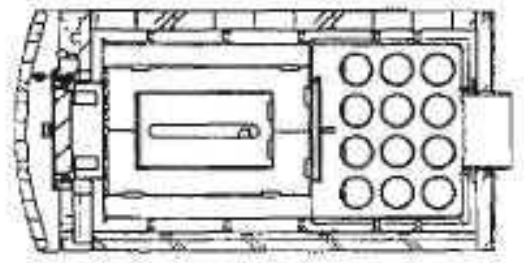

Solo Plus 60

\begin{tabular}{|c|c|c|c|c|}
\hline Technical Data & & 30 & 40 & 60 \\
\hline Maximum heavoutput & 8тU/hr & 100000 & 140,000 & 198,000 \\
\hline Firebox depth & inches & 21 & 21 & 21 \\
\hline Losd dor & in $x$ in & $10 \times 12$ & $10 \times 12$ & $10 \times 12$ \\
\hline irebox volume & cubls feet & 401 & 5.35 & 603 \\
\hline Whaxirnum woodienigth & incives & 20 & 20 & 20 \\
\hline Test pressure boller & $P S$ & 65 & 65 & 65 \\
\hline
\end{tabular}

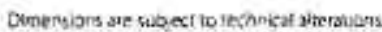

\begin{tabular}{|c|c|c|c|c|c|}
\hline \multicolumn{2}{|c|}{ Technicat Data } & & \multirow{2}{*}{$\frac{30}{45 \% / 2}$} & \multirow{2}{*}{$\frac{40}{46 \% 3}$} & \multirow{2}{*}{$-\frac{60}{504}$} \\
\hline Unit lengch & $\mathrm{x}$ & inches & & & \\
\hline Jnit width & $Y$ & inches & 21 & 25 & 25 \\
\hline Unit height & $z$ & inches & $55 \%$ & $55 \%$ & $55 \%$ \\
\hline \multicolumn{2}{|l|}{ Unit weight } & pounds & 1,080 & 1,180 & 1,230 \\
\hline \multicolumn{2}{|c|}{ Five colar size } & incties & 6 & 6 & 8 \\
\hline \multicolumn{2}{|c|}{ Height of fue rolar } & incties & $41 \%$ & $41 \times 4$ & $413 / 2$ \\
\hline
\end{tabular}

Siorage tank itial volume is 100 galloris/14,000 8T is 


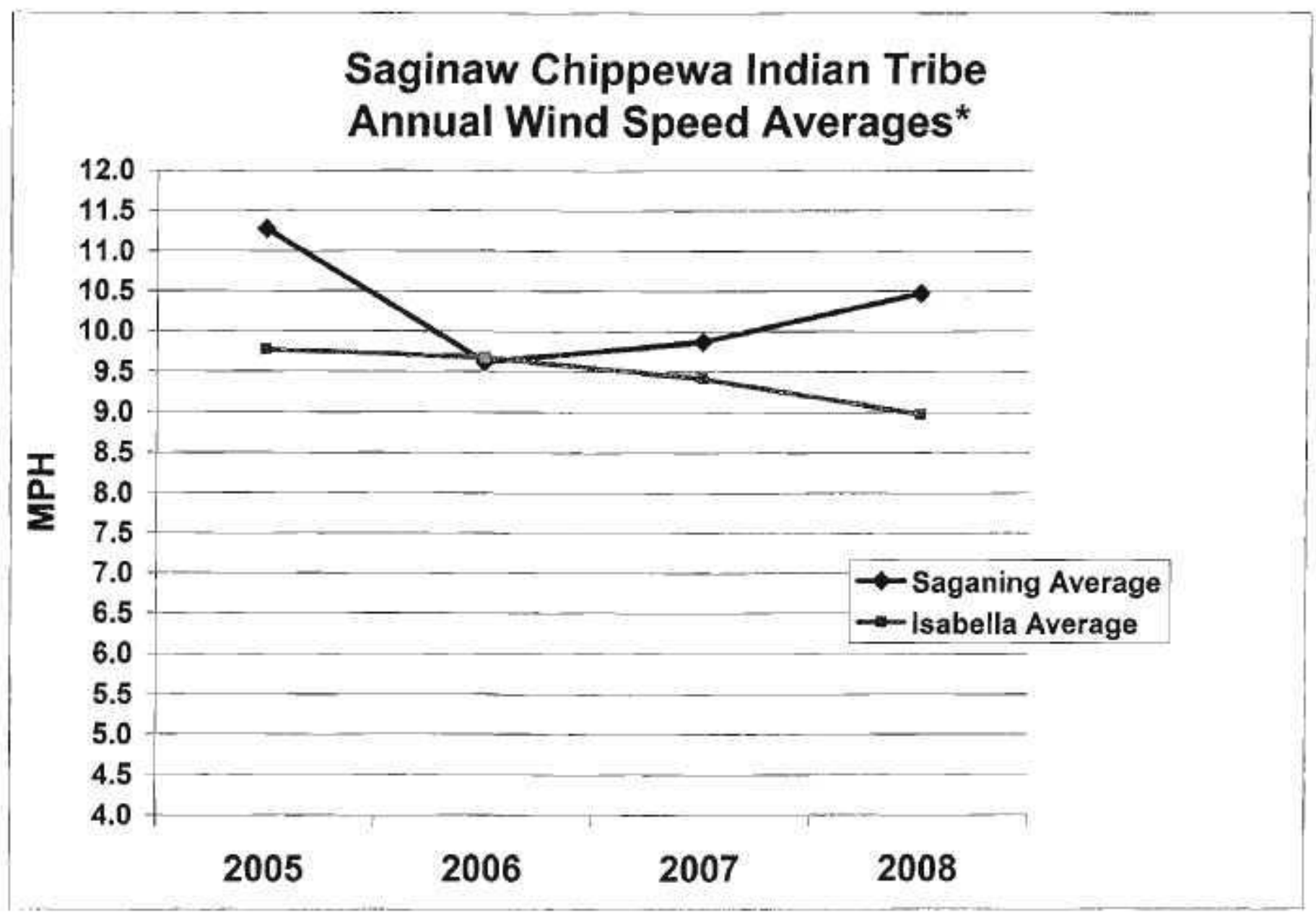




\section{Saganing Monthly Averages}

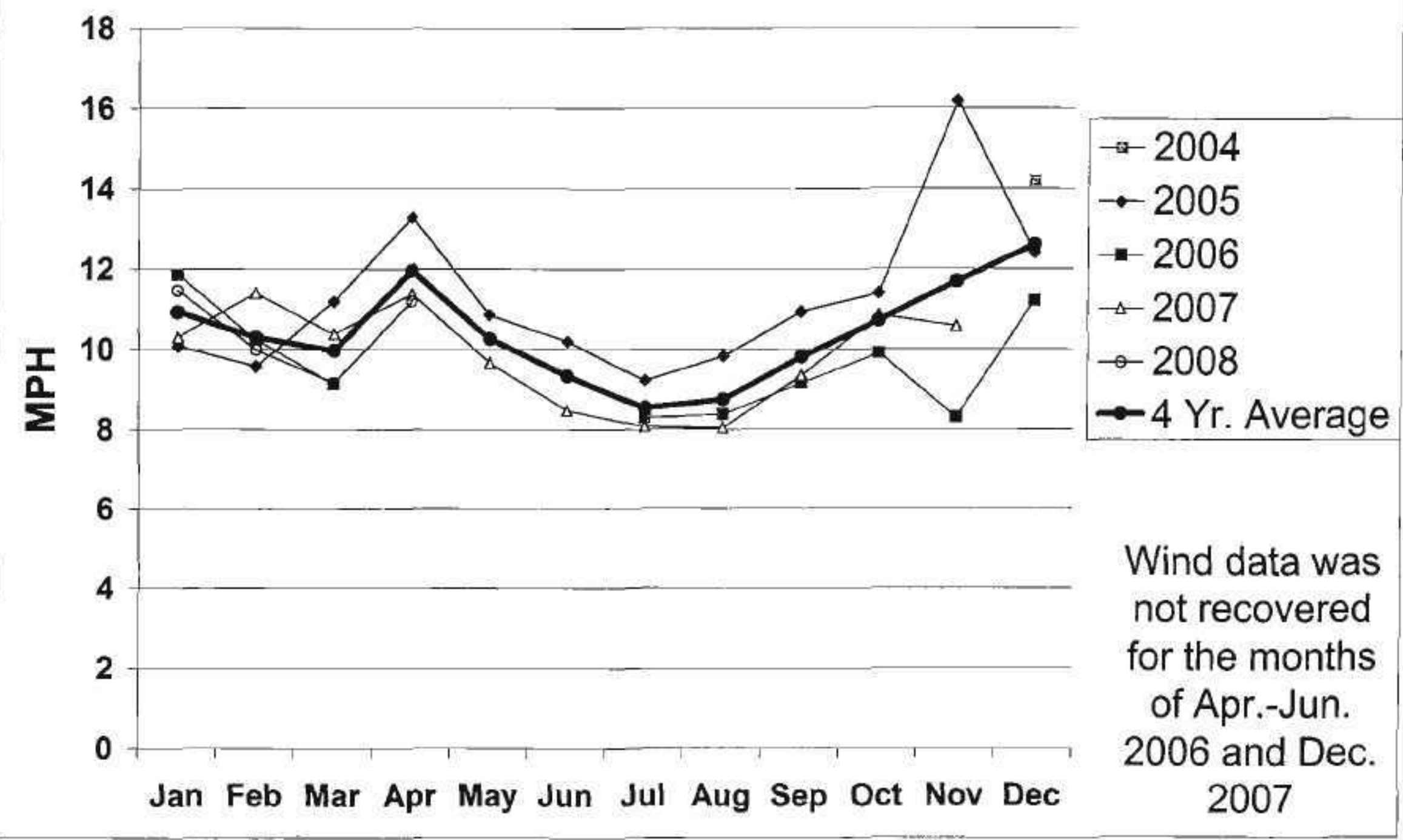


Site Information:

Project: New Project

Location:

Elevation:

\section{Sensor on channel 1:}

$\mathrm{NRG} \# 40$ Anem. $\mathrm{mph}$

Height: $\quad 50 \mathrm{~m}$

Serial \#: SN:
$12 / 15 / 2004$ to $4 / 27 / 2008$

Frequency Distribution Ch 1 SITE 5436

Saganing

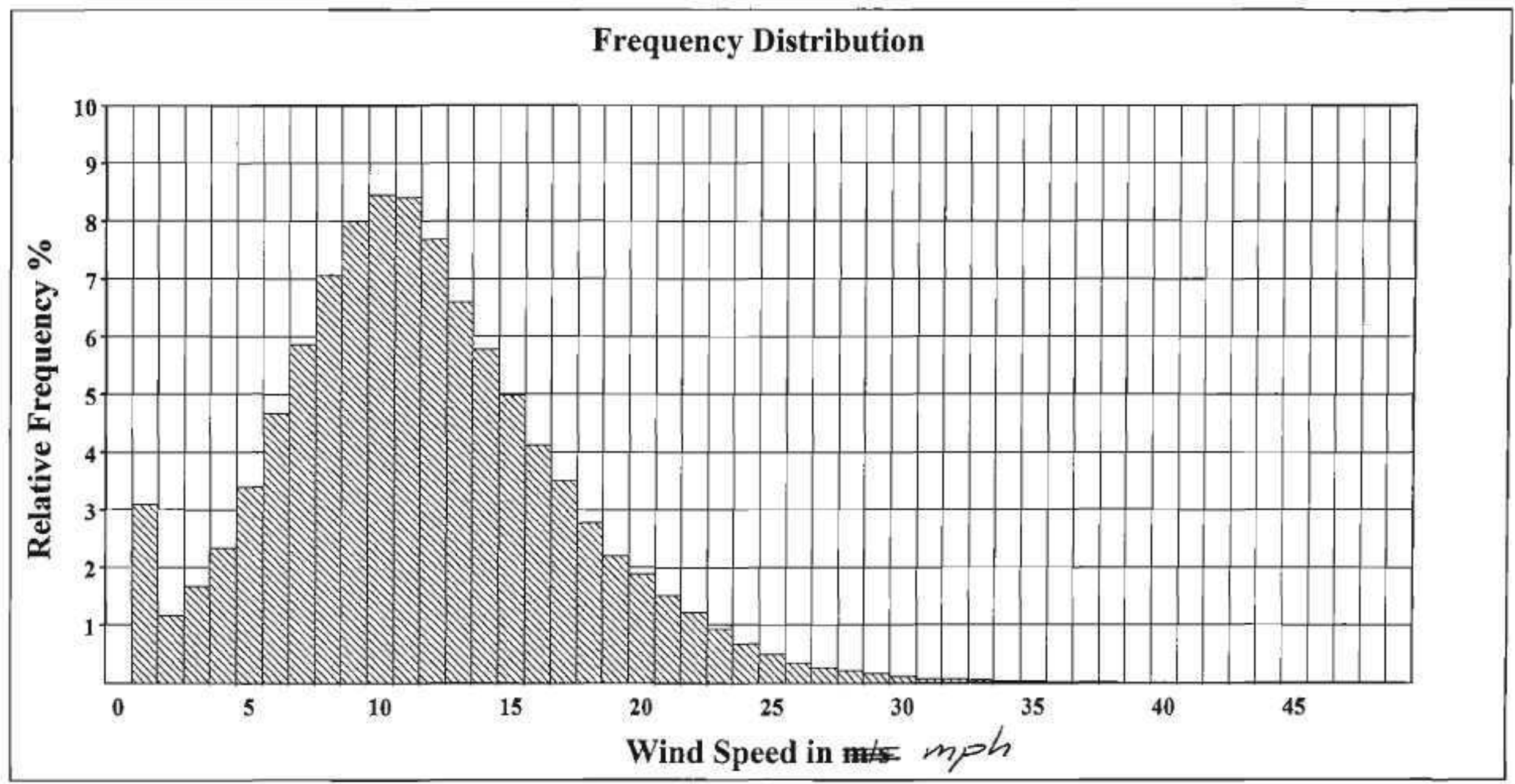




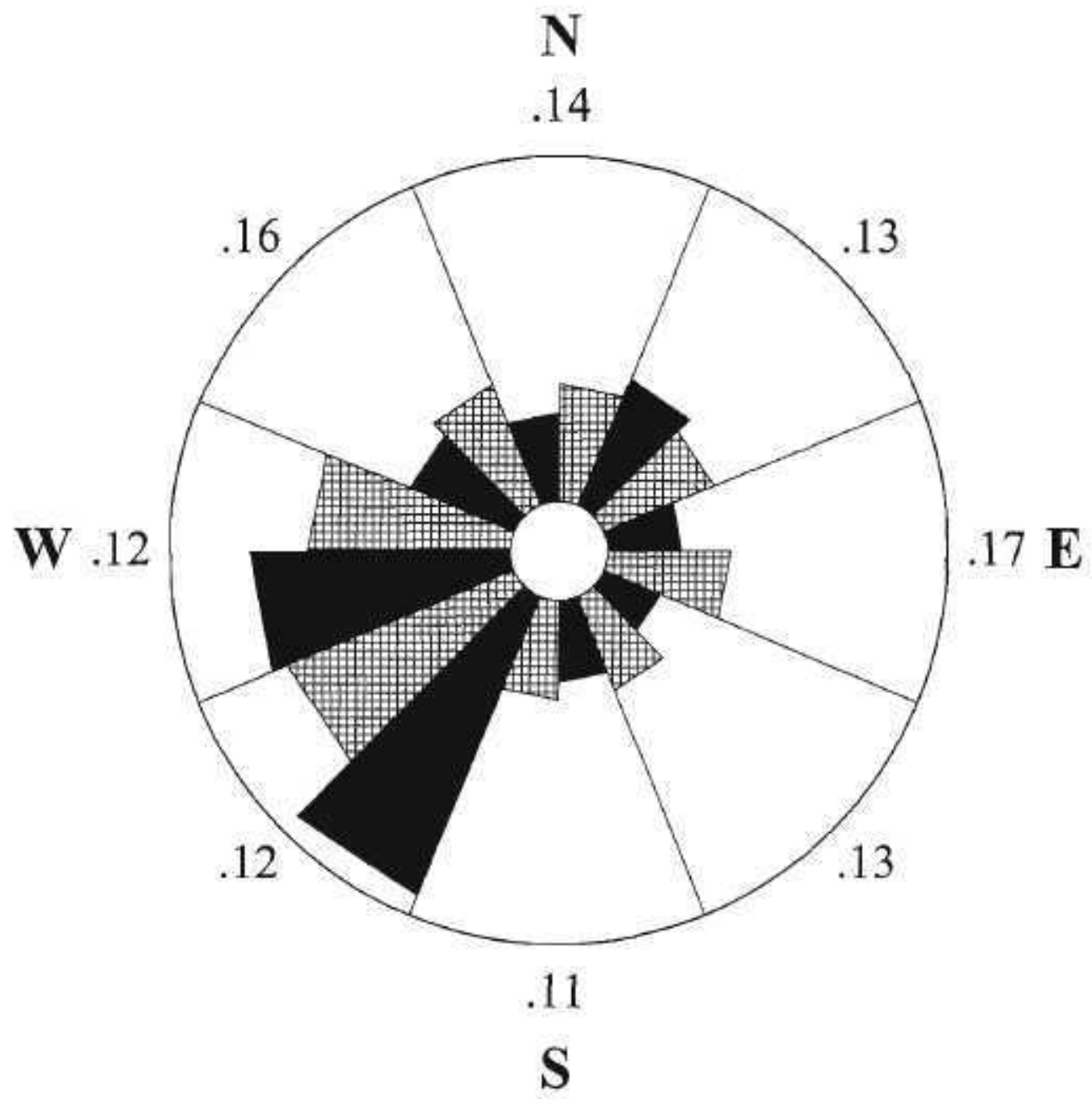

$12 / 15 / 2004$ to $4 / 27 / 2008$

Wind Rose Ch 1, 7

SITE 5436

Saganing

Site Information:

Project: New Project

Location:

Elevation:

Anemometer on channel 1:

NRG $\$ 40$ Anem, mph

Height: $\quad 50 \mathrm{~m}$

Serial \#: $\quad$ SN:

Vane on channel 7:

\#200P Wind Vane

Height: 0

Serial \#: $\quad \mathrm{SN}$ :

Outer Numbers are Average TIs

for speeds greater than $4.5 \mathrm{~m} / \mathrm{s}$

Inner Circle $=0 \%$

Outer Circle $=30 \%$

Percent of Total Wind Energy

Percent of Total Time

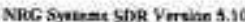




\section{Site Information:}

Project: Saginaw Chippewa Indian Tribe

Location:

Elevation:

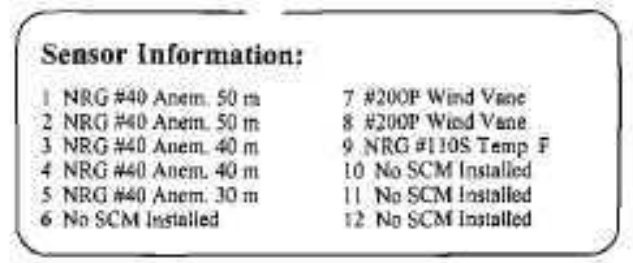

$12 / 15 / 2004$ to $4 / 27 / 2008$

\section{Summary Report}

SITE 5436

Sagaving

\begin{tabular}{|c|c|c|c|c|c|c|c|c|c|c|c|c|}
\hline Chasanel & 1 & 2 & 3 & 4. & 5 & & 7 & 8 & 9. & & & \\
\hline Helght & $50 \mathrm{~m}$ & $50 \mathrm{~m}$ & $40 \mathrm{~m}$ & $40 \mathrm{~m}$ & $30 \mathrm{~m}$ & $x+1$ & 0 & 0 & 0 & $\ldots$ & $\cdots$ & $\ldots$ \\
\hline Units & $m p h$ & $\mathrm{mph}$ & mph & mphi & $\mathrm{mph}$ & $\cdots$ & deB & deg & $\mathrm{F}$ & $\cdots$ & $\rightarrow$ & $\cdots$ \\
\hline Isternalt with Valld Data & 144064 & 144064 & 144064 & 144054 & 154064 & & 144064 & 144064 & 144064 & & & \\
\hline Avernge Fhatered Data & 11.47 & 11.41 & 10.54 & 10.49 & 985 & & 255.6 & 251.1 & 46.61 & & & \\
\hline Average for All Data & 11.67 & 11.41 & 10.58 & 10.49 & 9.85 & & 255.6 & 2511 & 46.61 & & & \\
\hline Mia Interyat Average & 0.8 & 0.8 & 0.8 & c.8 & 0.8 & & & & .12 & & & \\
\hline Date of Min Iaterval & $12 / 26 / 2004$ & $12 / 17 / 2004$ & $12 / 262004$ & $12 / 26 / 2004$ & $12 / 26 / 2004$ & & & & $1 / 25 / 2007$ & & & \\
\hline Time of Min Interval & $2=10: 00 \mathrm{AM}$ & 12:00:00 PM & $2: 10: 00 \mathrm{AM}$ & $2: 1000 \mathrm{AM}$ & $150.00 \mathrm{AM}$ & & & & $9.0000 \mathrm{PM}$ & & & \\
\hline Mex Interval Average & 45.4 & 46 & 44.4 & 67.9 & 42,7 & & & & 99.1 & & & \\
\hline Date of Max Interval & $13 / 13 / 2005$ & $11 / 13 / 2005$ & $11 / 13 / 2005$ & $3 / 3 / 2007$ & $11 / 13 / 2005$ & & & & $8 / 12906$ & & & \\
\hline Time of Max Interval & $13000 \mathrm{PM}$ & $1.3000 \mathrm{PM}$ & 1:30:00 $\mathrm{PM}$ & H1-3000 AM & $130.00 \mathrm{PM}$ & & & & $2: 10.00 \mathrm{FM}$ & & & \\
\hline Average interval SD & {$[.5 \mathrm{t}$} & 1.54 & 1.52 & 1.6 & 1.58 & & 9.76 & 8.92 & 0.11 & & & \\
\hline Min Sample & 0.8 & 0.8 & 08 & 0.8 & 0.8 & & & & .588 & & & \\
\hline Date of Mis Skmple & $1277 / 2004$ & $12 / 17 / 2004$ & $12 / 17 / 2004$ & $12 / 17 / 2054$ & $12 / 172004$ & & & & $11 / 26 / 2007$ & & & \\
\hline Time of Min Sample & $1000.00 \mathrm{AM}$ & 10:00:00 AM & $940: 00 \mathrm{AM}$ & 9:40:00 AM & 7.30:00 AM & & & & $12.00000 \mathrm{AM}$ & & & \\
\hline Mex Semple & 62.5 & 63582 & 61.5 & 20147.8 & 59.8 & & & & 99.6 & & & \\
\hline Date of Max Sample & $11 / 162005$ & $3 \pi / 200 ?$ & 792007 & $3 / 3 / 2007$ & $1 \mathrm{t} / 13 / 2005$ & & & & $81 / 2006$ & & & \\
\hline Tine of Mar Saraple & $3.50 .00 \mathrm{AM}$ & $7: 50,00 \mathrm{PM}$ & $320.00 \mathrm{PM}$ & $11 \cdot 30.00 \mathrm{AM}$ & $5,00,00 \mathrm{PM}$ & & & & $2: 1000 \mathrm{PM}$ & & & \\
\hline Aserage Interval TI & 0.14 & 0.15 & 0.15 & 0.16 & 0.17 & & & & & & & \\
\hline Wlad Speed Direction & & & & & & & sw & sw & & & & \\
\hline
\end{tabular}




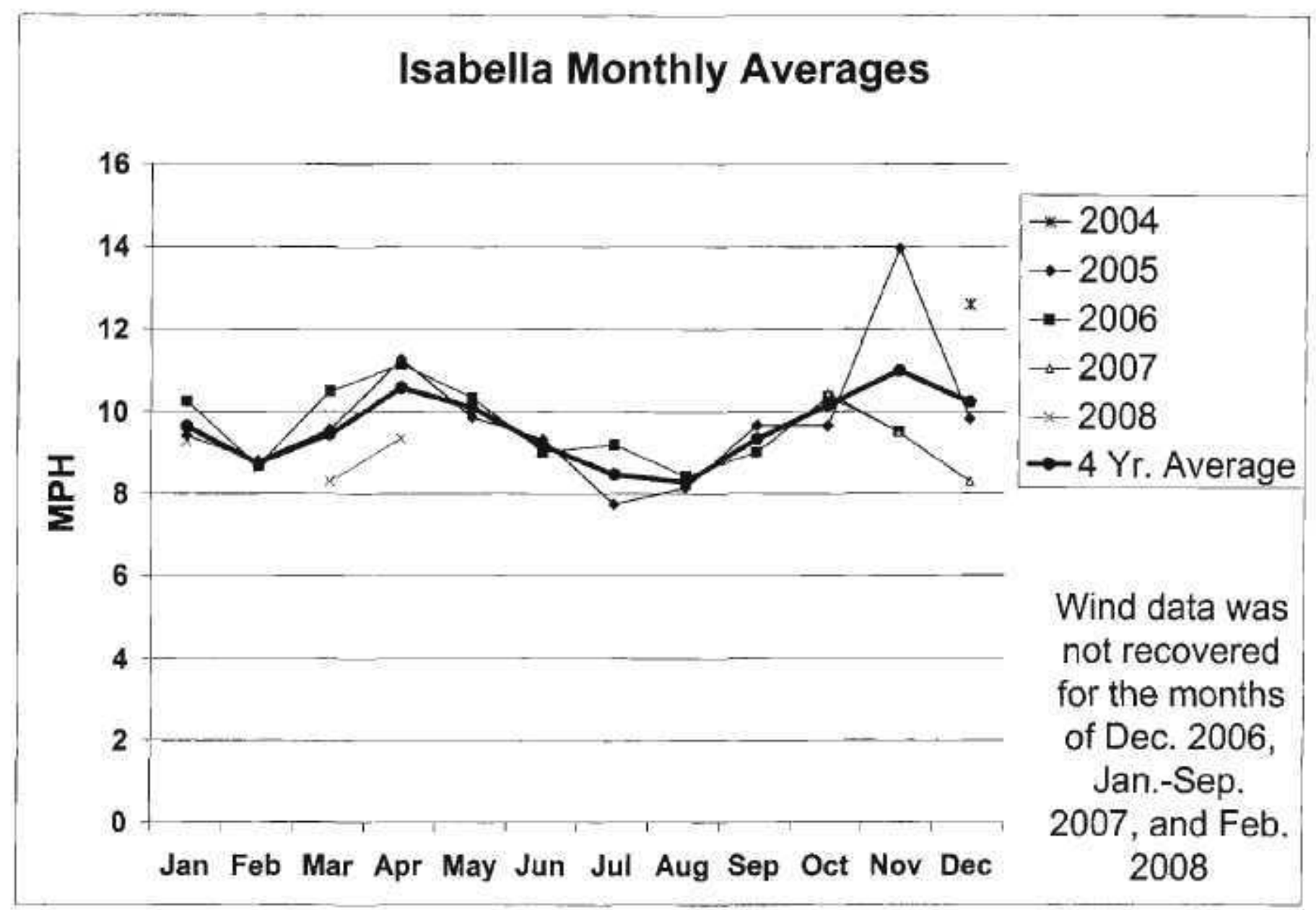




\section{Site Information:}

Project: New Project

Location:

Elevation:

\section{Sensor on channel 1:}

NRG \#40 Anem. mph

Height: $\quad 50 \mathrm{~m}$

Serial \#: $\quad$ SN:

\section{$12 / 15 / 2004$ to $4 / 23 / 2008$}

Frequency Distribution Ch 1 SITE 5435

Isabella

\section{Frequency Distribution}

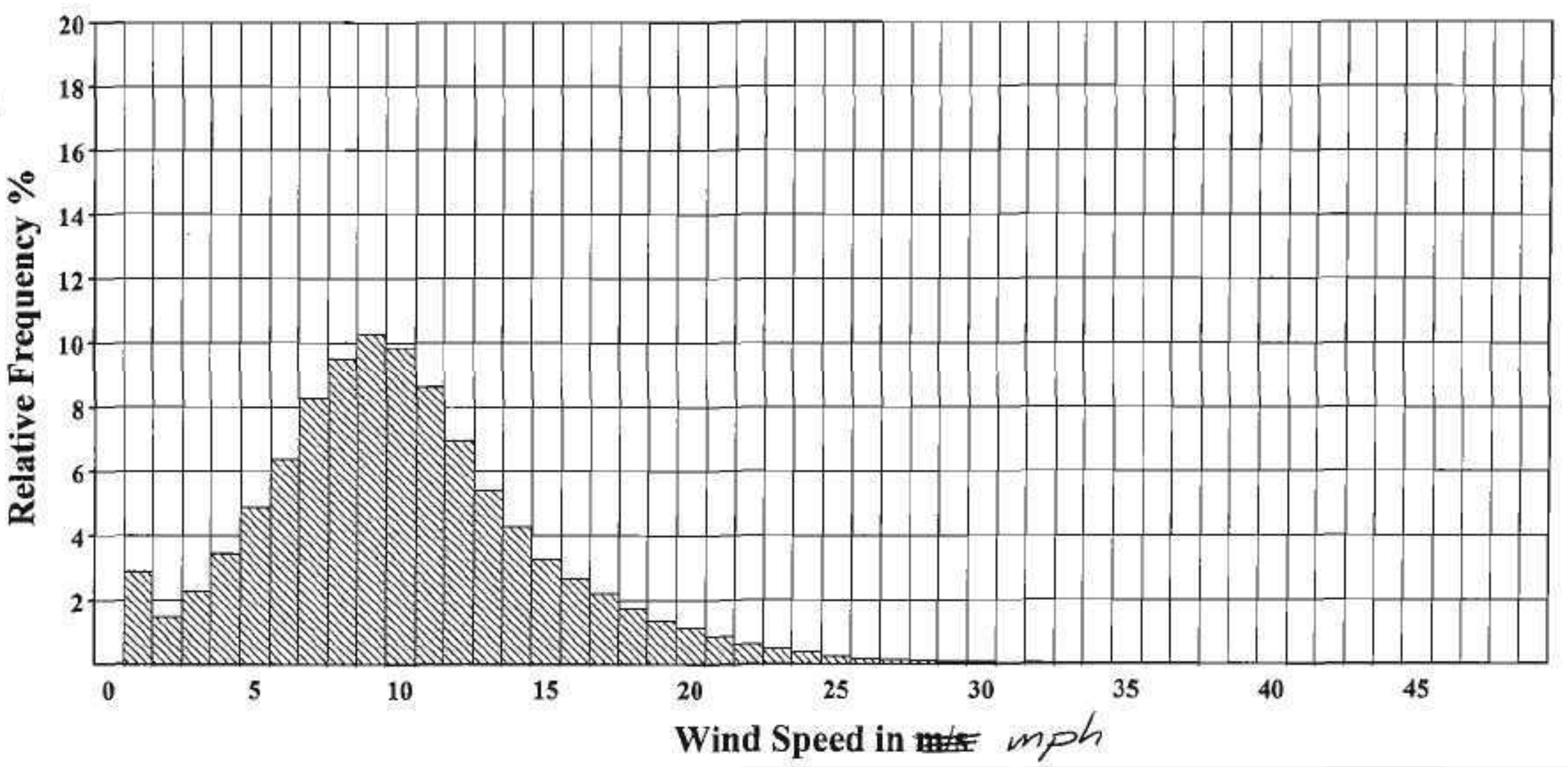




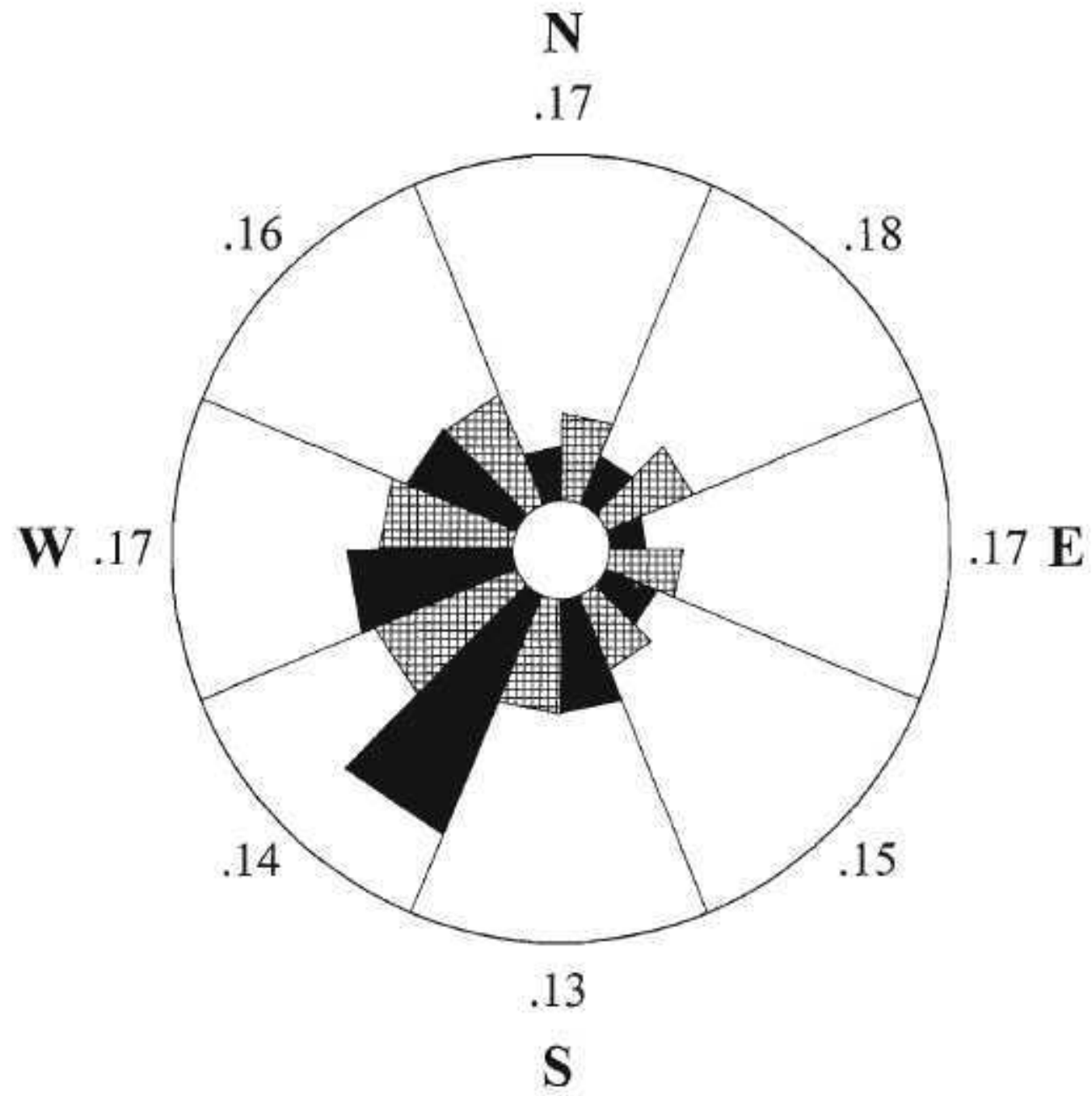

$12 / 15 / 2004$ to $4 / 23 / 2008$

Wind Rose $\mathrm{Ch} 1,7$ new SITE 5435

Isabella

Site Information:

Project: New Project

Location:

Elevation:

Anemometer on channel 1:

NRG $\# 40$ Anem, mph

Height: $\quad 50 \mathrm{~m}$

Serial \#: $\mathrm{SN}$ :

Vane on channel 7:

\#200P Wind Vane

Height: 0

Serial \#: SN:

Outer Numbers are Average T7s for speeds greater than $4.5 \mathrm{~m} / \mathrm{s}$.

Inner Circle $=0 \%$

Outer Circle $=40 \%$

Percent of Total Wind Energy

Percent of Total Time

NRE Synteres SDQ Vertion o.13 


\section{Site Information:}

Project: Saginaw Chippewa Indian Tribe

Location:

Elevation:
Sensor Information:

1. NRGG A 10 Anesa $50 \mathrm{~m}$

2 NRO b40.Anern, $50 \mathrm{~m}$

3 NRG 140 Anern. 40 en

4 NRG 440 Aner: $40 \mathrm{~m}$

5 NRO 4040 Aem. 30 in

6 No SCM Installed.
7 *200\% Wind Vane

8 H200P Wind Vane

9 NRQ 110 S Temp F

10 No SCM Instailed

il NeM Instaled

12 No SCM Installed
$12 / 15 / 2004$ to $4 / 23 / 2008$

Summary Report SITE 5435

Isabella

\begin{tabular}{|c|c|c|c|c|c|c|c|c|c|c|c|c|}
\hline Channel & 1 & 2 & 3) & 4 & 5 & & 7 & 8 & 9 & & & \\
\hline Height & Som & $50 \mathrm{~m}$ & $40 \mathrm{~m}$ & $40 \mathrm{~m}$ & $30 \mathrm{~m}$ & $m$ & o) & of & 0 & w.... & $\cdots$ & $\ldots$ \\
\hline Units & miph & mpis & $\mathrm{mpb}$ & mph & mohy & - & deg. & dep. & $\mathrm{F}$ & $+\cdots$ & $\cdots$ & $\cdots$ \\
\hline Iatervals with Vatid Datu & 118504 & 118504 & 118504 & 118504 & 118504 & & {$[18504]$} & 118506 & 118508 & & & \\
\hline Averige Fittered Data & 10.03 & 9.67 & 9.14 & 9.08 & 83 & & 2508 & 253.43 & .2464 & & & \\
\hline Average for All Data & 1003 & 9.67 & 9.14 & 9.08 & 8.3 & & 250.8 & 253.43 & 24.64 & & & \\
\hline Min Interval Averxge & 0.8 & D.8 & 0.8 & 0.8 & 0.8 & & & & -121.8 & & & \\
\hline Date of Shin Luterval & $12 / 2712004$ & $12262 / 2 n 04$ & $12 / 27 / 2004$ & $12 / 27 / 2004$ & $12 / 27 / 2004$ & & & & $2 / 23 / 2007$ & & & \\
\hline Thene of Min Inieryd & $8.1000 \mathrm{AM}$ & $1: 40: 00 \mathrm{AM}$ & $10.10,00 \mathrm{AM}$ & $10: 10: 00 \mathrm{AM}$ & $9.50 .00 \mathrm{AM}$ & & & & 5:40:00 PM & & & \\
\hline Max Iaterval Average & 42.9 & 429 & 40.9 & 41.3 & 397 & & & & 94.1 & & & \\
\hline Date of Max Interval & $3 / 1 / 2006$ & $313 / 2006$ & $3 / 13 / 2006$ & $3 i 13 / 2006$ & $3 / 23 / 2006$ & & & & $6 / 27 / 2005$ & & & \\
\hline Time of Mnx Interval & 5:00:00 PM & $500-00 \mathrm{PM}$ & $63000 \mathrm{PM}$ & $6.30 \mathrm{mPM}$ & $6: 30: 00 \mathrm{PM}$ & & & & $2.2000 \mathrm{PM}$ & & & \\
\hline Aversze Iaterval SD & 1.63 & 1.7 & 1.64 & 1.64 & 1.63 & & 11 & 11.73 & 023 & & & \\
\hline Min Sazple & 0.8 & 0.8 & 0.8 & 0.8 & 0.8 & & & & -122.5 & & & \\
\hline Date of Min Sample & 127172004 & $12 / 172004$ & $12 / 17 / 2004$ & $12 / 17 / 2004$ & $12 / 17 / 2004$ & & & & $2 / 23 / 2007$ & & & \\
\hline Tlen or Min Sample & $9.10: 60 \mathrm{AM}$ & $9.10 .60 \mathrm{AM}$ & $9: 10: 00 \mathrm{AM}$ & $91000 \mathrm{AM}$ & $9: 00.00 \mathrm{AM}$ & & & & $6: 10: 00 \mathrm{PM}$ & & & \\
\hline Max Sampic & 68.4 & 68.4 & 66.6 & 67.5 & 65 & & & & 94.8 & & & \\
\hline Date of Max Snmple & $7 / 1720006$ & $7 / 17 / 2006$ & 73712006 & $7 i 17 / 2006$ & $717 / 2006$ & & & & $6227 / 2005$ & & & \\
\hline Time of Max Sample & $5.10500 \mathrm{PM}$ & $5: 10.00 \mathrm{PM}$ & 5:10:00 PM & $5: 10.00 \mathrm{PM}$ & $5.1000 \mathrm{PM}$ & & & & $2.1000 \mathrm{PM}$ & & & \\
\hline Average faterval T1 & 0.17 & 0.19 & 0.19 & 0.19 & 0.21 & & & & & & & \\
\hline Wind Speed Direetion & & & & & & & sw & $w$ & & & & \\
\hline
\end{tabular}


Appendix E. Saganing Single Commercial Wind Turbine Pro Forma's 
SAGINAW CHIPPEWA

WIND TURBINE GENERATORS

^^GANING WIND PROJECT
183 meter

$1500 \mathrm{~kW}$
PRO FORMA CASH FLOW PROJECTIONS

\author{
ASSUMPTIONS \\ Total Cost: \\ Debt: \\ Equity: \\ $75 \%$ \\ $25 \%$ \\ Wind Power Purchase Electric Rate or Offset: \\ Electric escalation rate: \\ Annual Output kwhilyr. WTG. \\ Masntenance feelyr MTG: \\ All-Risk Insurance/\$100; \\ Ajr Pollution Emission Credits/kW-hr \\ Cost per WTG \\ Total Cost
}

Net Wind Output

Rotor Dia

$\$ 1.933$ Finance Term:

Interest Rate:

Federal Tax:

Federal incentive (10 yrs):

Federai Tax Credit:

O\&M \& REPI Escalation rate:

Mgt. feeryt MTT:

Utility/Sub, feetyr.NTTG.

Land Rentlyr. NTG:

Local Eroperty Tax:
Sep-08

$613 \mathrm{kWh}$ rstys/m2

a3 meter

20 years

$6.00 \%$ year

O\% per annum

$\$ 0.0200$ per $k W-h r$

$\$ 0.000$

$2.00 \%$

$\$ 8.000$

$\$ 1,200$

50.005

$2010 \quad 2011$

2009

0.0700

3316077

232125

66322

16580

315,027

EXPENSES

- Rent

ivicuragement

Maintenance

Local Taxes

All-risk Insurance

Performance Insur.

Utility \& Substation

TOTAL EXPENSES

-

NET REVENUE

Debt Service

Total Debt \& O\&M Expense

CASH FLOW

Debt Coverage Ratio

Cost/ kW-hr, (pre REP)

CosthkW-hr With REPI

Principal

Interest

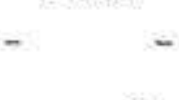

6,000

28,000

0

5,438

0

1,200

40,638

274,390

189,626

230.264

84,763

1.45

$\$ 0.069$

$\$ 0.049$

59,126

130,500

FINANCIAL SUMMARY DATA

\begin{tabular}{rr}
2010 & 2011 \\
2 & 3 \\
0.0728 & 0.0757 \\
3316077 & 3316077 \\
241410 & 251067 \\
67648 & 69001 \\
16912 & 17250 \\
325,970 & 337,318 \\
\hline &
\end{tabular}

2012
4
0.0787
3316077
261109
70381
17595
349,086

10

6.120

28.560

0
5.546

0
1.224

41,450

284,520

189,626

231,077

94,894

1.50

$\$ 0.070$

$\$ 0.049$

62,674

126,952

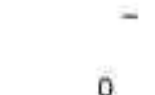

$\begin{array}{r}0 \\ 6,242 \\ 29,131 \\ 0 \\ 5,657 \\ 0 \\ 1,248 \\ 42,279 \\ \hline\end{array}$

295,039

189,626

231,906

105,412

1.56

$\$ 0.070$

$\$ 0.049$

66,434

123,192

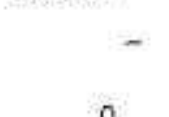

2013
5
0.0819
3316077
271554
71789
17947
361,290

2014
6
0.0852
3316077
282416
73224
18306
373,946

2015

0.0886

3316077

293713

74689

18672

387,074

$\begin{array}{rr}0 & 0 \\ 6,367 & 6,495 \\ 29,714 & 30,308 \\ 0 & 0 \\ 5,770 & 5,886 \\ 0 & 0 \\ 1,273 & 1,299 \\ 43,125 & 43,987\end{array}$

0
6,624
30,914
0
6,003
0
1,325
44,867

$305,961 \quad 317,302$

329,079

341,309

$\begin{array}{ll}189,626 & 189,626 \\ 232,751 & 233,614\end{array}$

189,626

189,626

234,493

235,391

$116,334 \quad 127,676$

139,453

151,683

$1.61 \quad 1.67$

1.74

$\$ 0.070 \quad \$ 0.070$

$\$ 0.07 t$

1.80

$\$ 0.071$

$\begin{array}{ll}\$ 0.049 & \$ 0.049\end{array}$

$\$ 0.049$

$\$ 0.048$

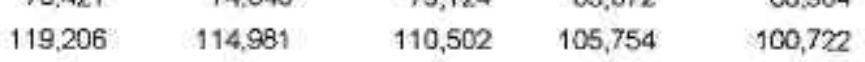

$\begin{array}{lllll}119,206 & 114,981 & 110,502 & 105,754 & 100,722\end{array}$

\section{BENEFIT/COST RATIO \\ PV of Benefits w/REPI}

PV of Costs

20 Y year "Cash on Cash"

NPV Electric Cost/kwh

Discount Rate

354,010

189,626

236,306

164,383

1.87
.871

$\$ 0.048$

$\$ 2,891,725$ Net Present Vaiue (25 yr)

$\$ 7,317,435$

$\$ 4,425,710$

$9.46 \%$ (net revenue/total cost)

$18.37 \%$

$\$ 0.045$ 
Appendix F. Saganing Wind Farm Pro Forma's 


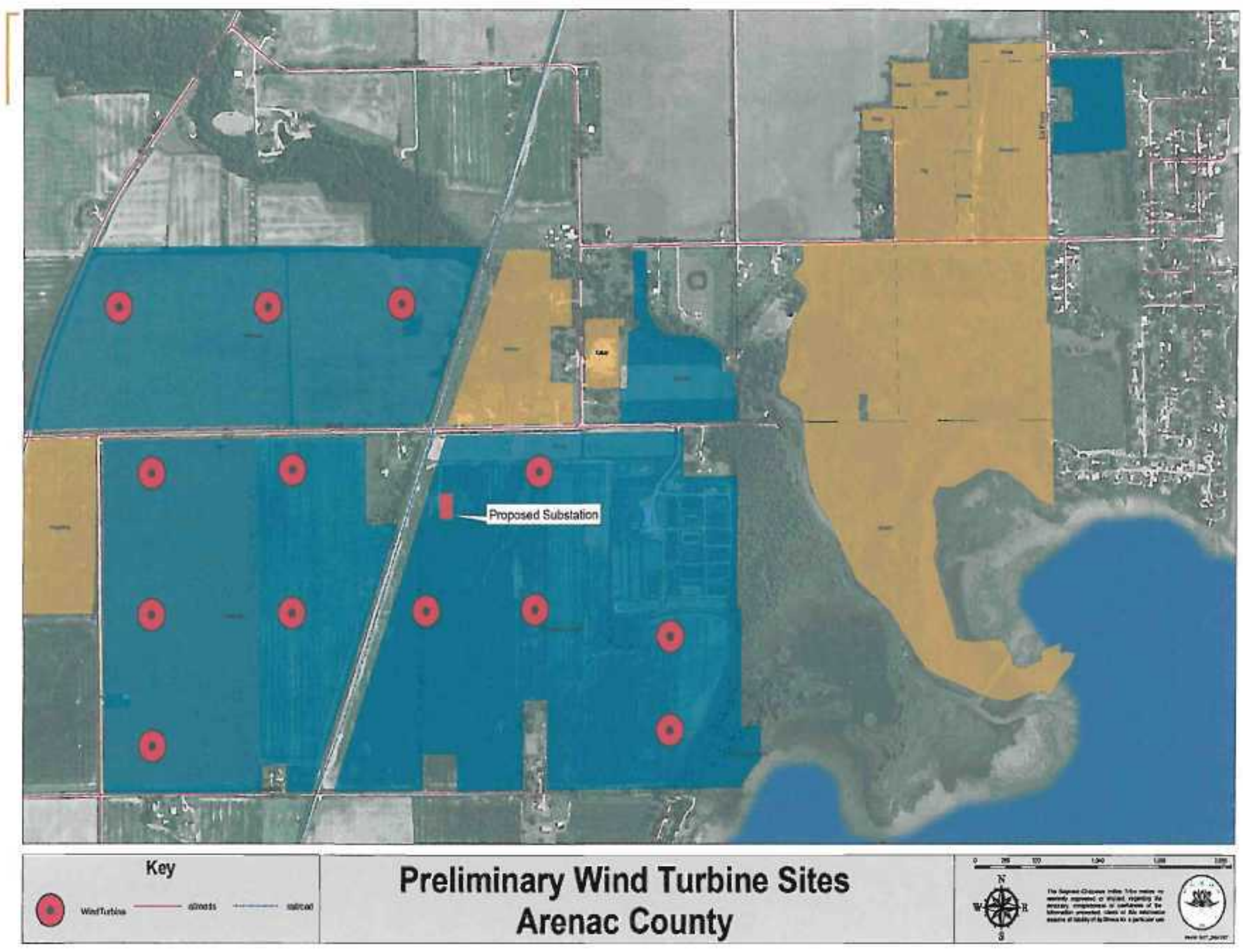




\section{Saganing Wind Project Economics:}

13 Wind Turbines

1. Total Cost: $\$ 35$

Million

Gross Income

per year $\$ 4$

million

-11 Cost of

Electricity 4.5

cents per kWh

Net Present

Value: $\$ 41$

million

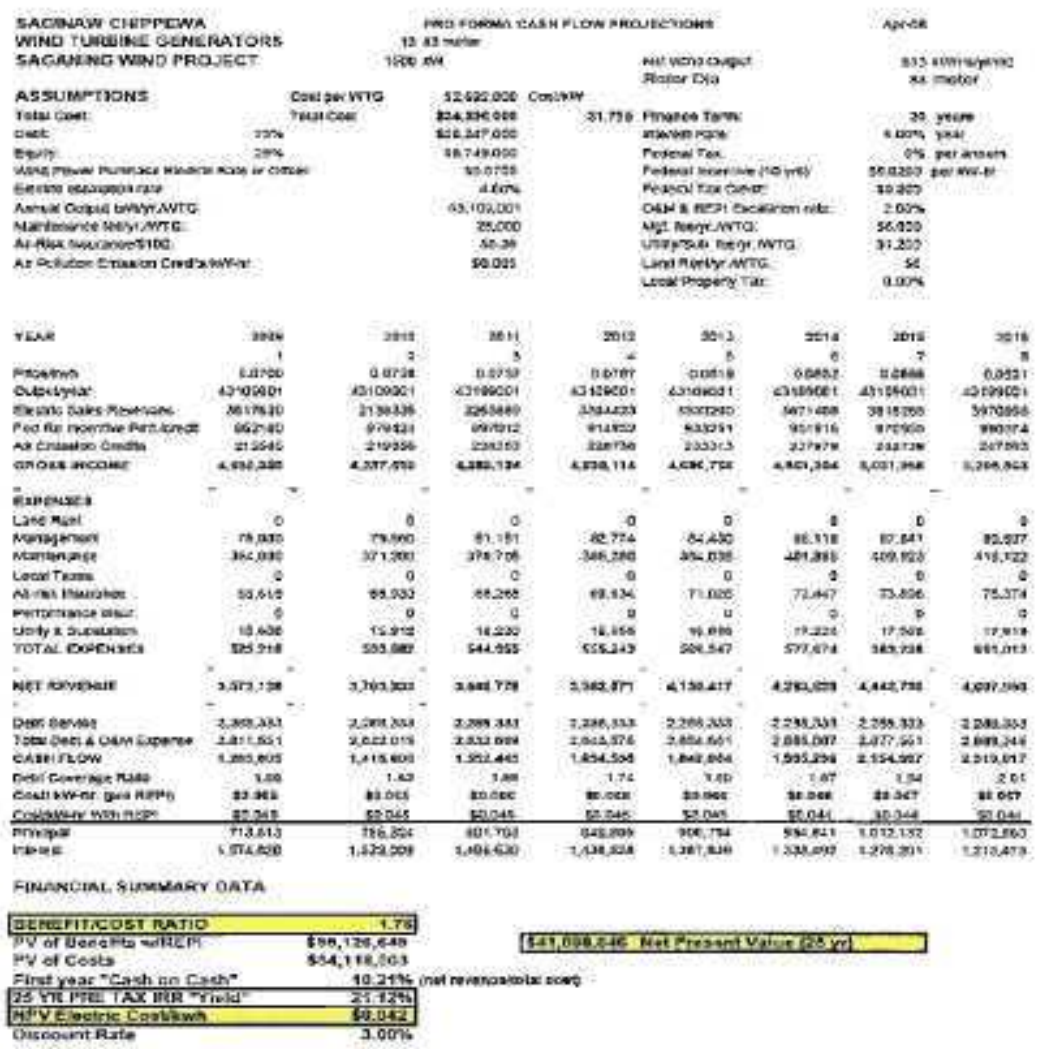


Appendix G. Isabella Wind Project Pro Forma's 


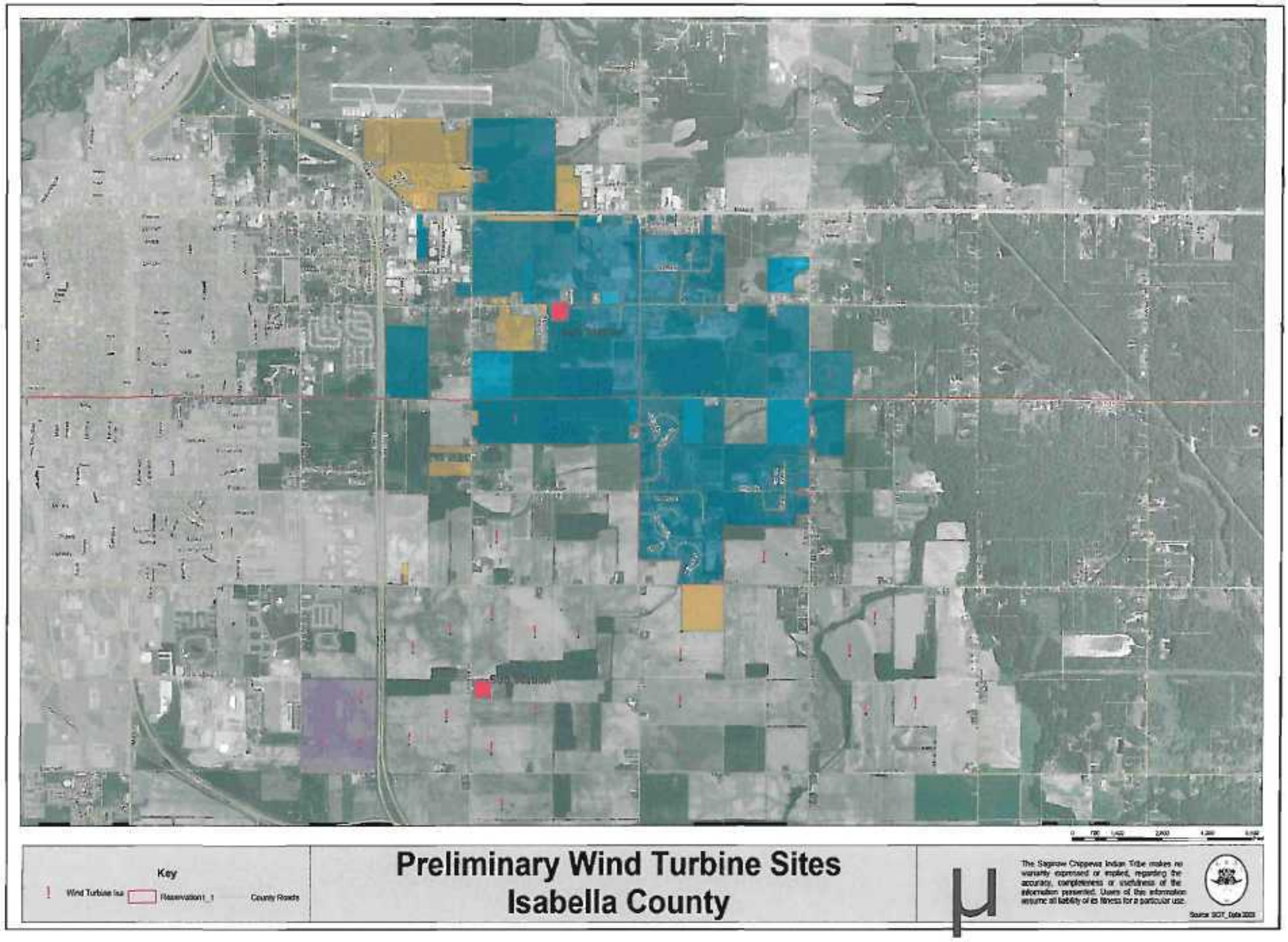




\section{Isabella Wind Project:}

19 Wind Turbines

- Total Project Cost: \$49 Million

- Gross Income per year: \$5.1 million

- Cost per kWh: 5.7 cents / kWh

Net Present

Value: $\$ 39.3$

million
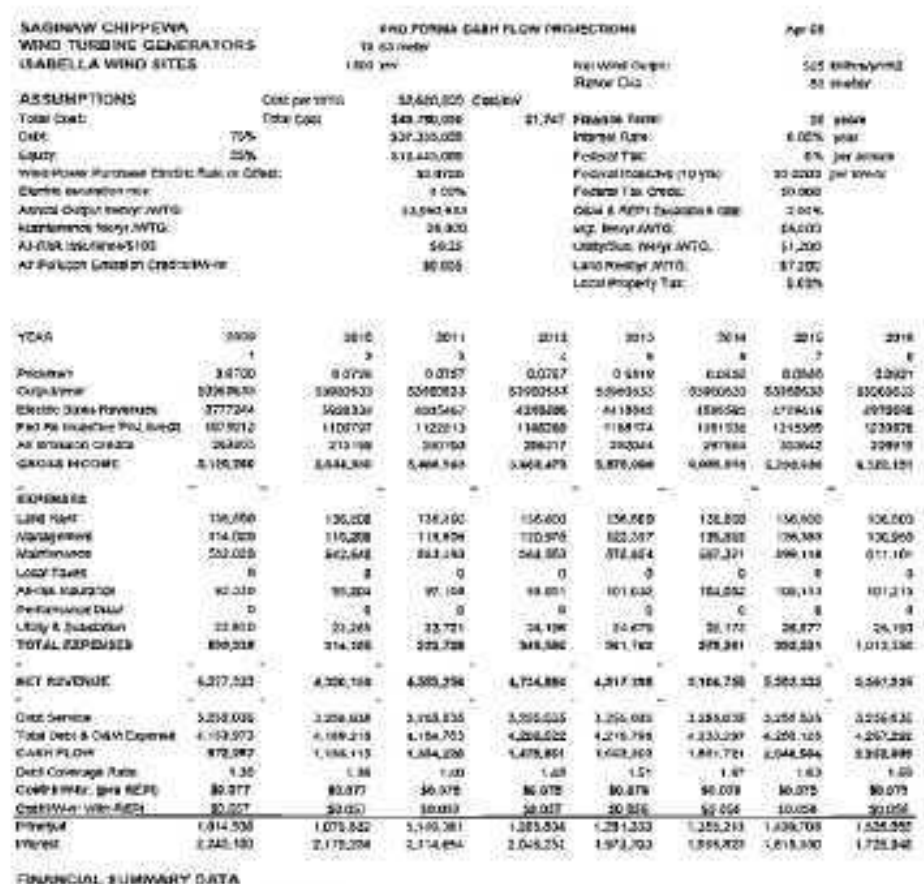

Fondroint sulambre onTA

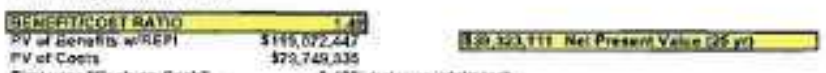




\section{Wind Power Economic Sensitivity Analysis}

Net Present Value (NPV) From Generating approximately 100\% of Annual Electric Consumption of 51 million $\mathrm{kWhr} / \mathrm{yr}$. Assumes 7 cent per $\mathrm{kW}$-hr cost avoidance, with avoided electric costs projected to increase either $2 \%, 4 \%$ or $6 \%$ over a 25 year period. (see attached preliminary pro forma spreadsheets)

Saganing: 13 Wind Turbines - Project Cost - $\$ 34,996,000$ Avoided

Cost Inflation

$2 \%$

$4 \%$

$6 \%$
NPV(25 yr)

$\$ 23,847,290$

$\$ 41,008,646$

$\$ 64,160,560$

Isabella: 19 Wind Turbines - Project Cost - $\$ 49,780,000$

$2 \%$

$4 \%$

$6 \%$
NPV (25 yr)

$\$ 17,875,602$

$\$ 39,323,111$

$\$ 68,302,951$ 
Appendix H. Biomass Stage I and II Financial Study 


\section{Power Plants Can Look Good!!}

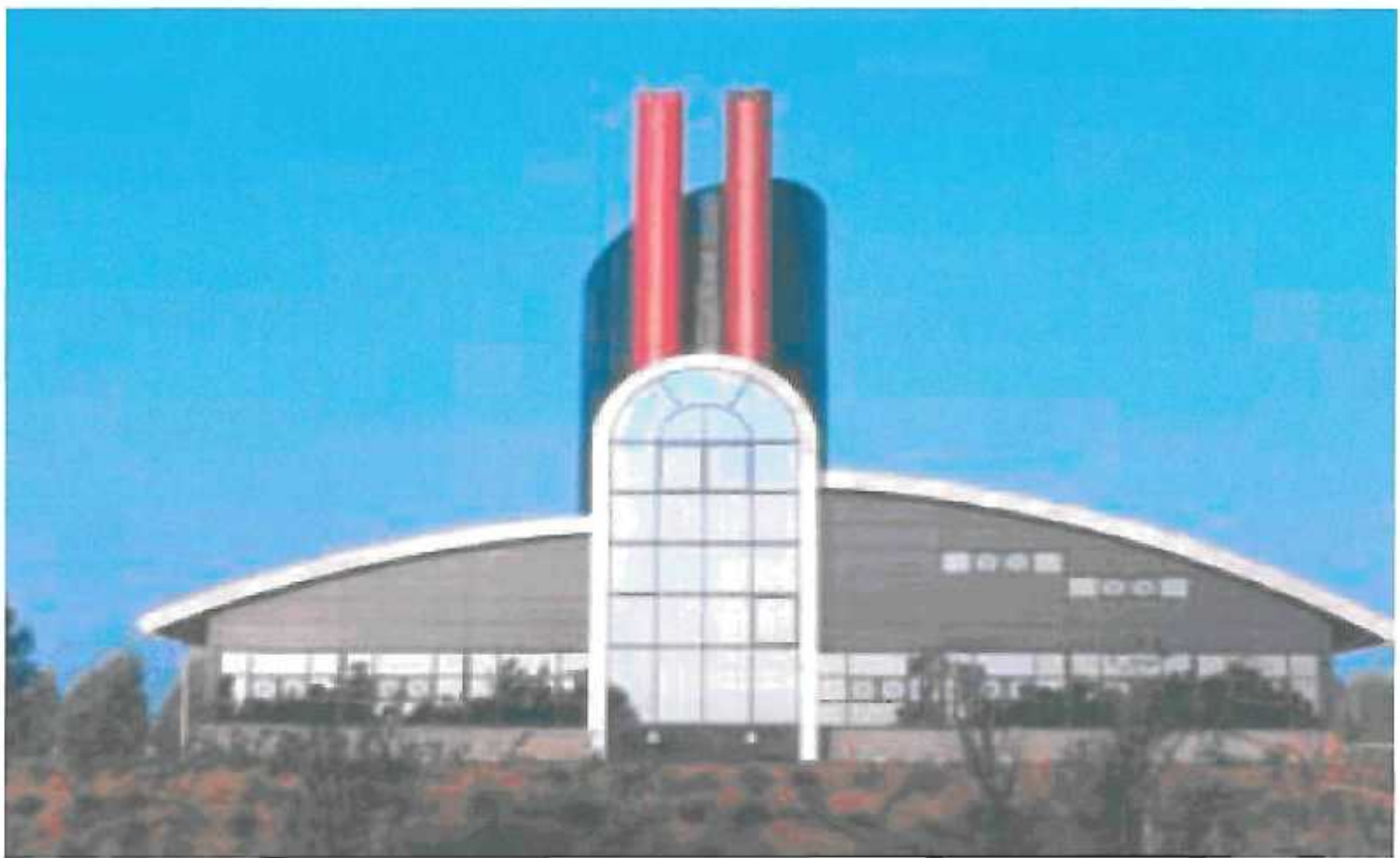




\section{Wood Chip Biomass Heat Plant (courtesy of Force Technology A. Evald)}

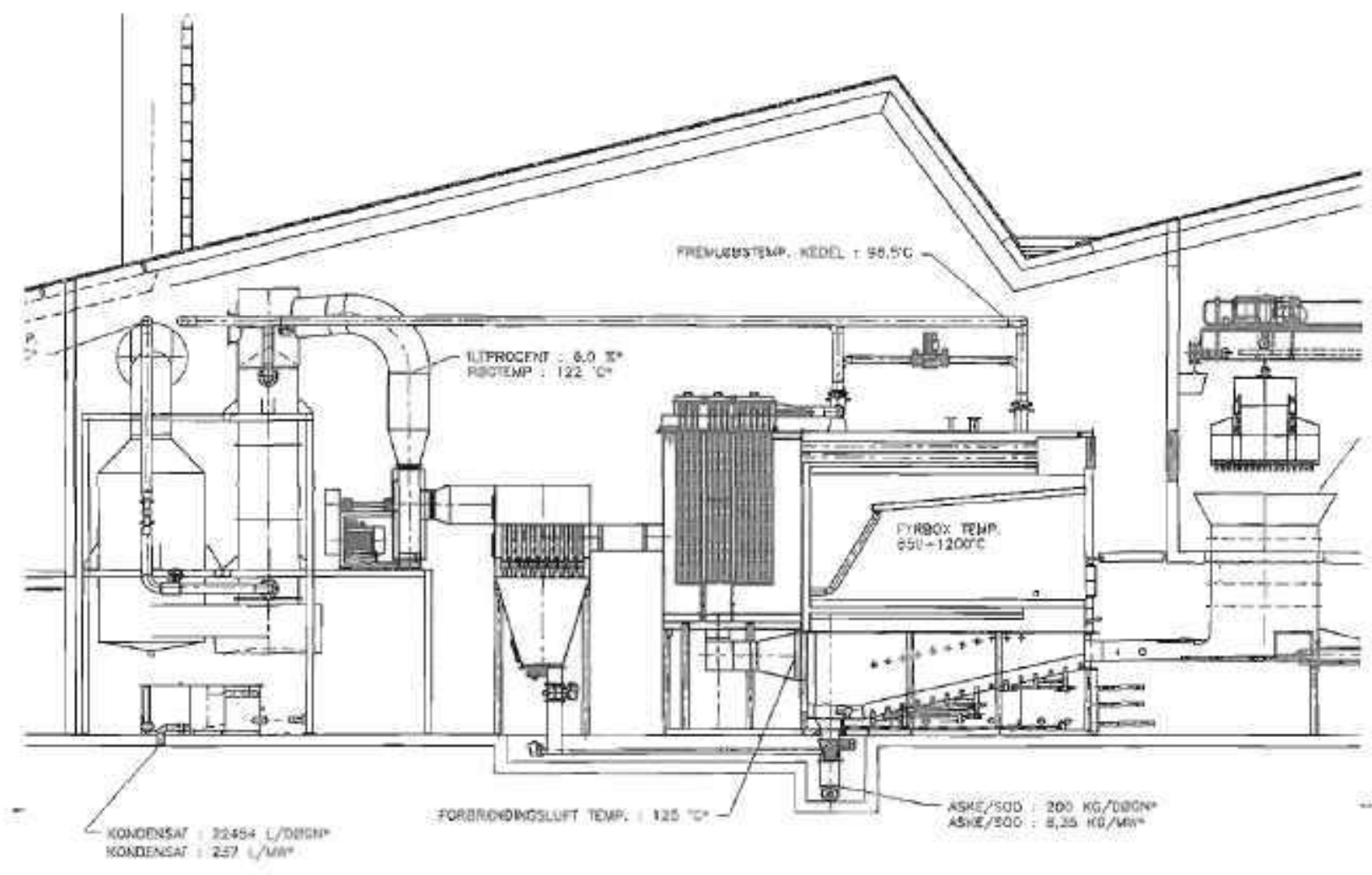




\section{Biomass \\ District \\ Heat \\ Preliminary \\ Concept \\ Plan}

Stage If Central Commercial and Public District

\section{Stage II:}

Residential and remote facilities

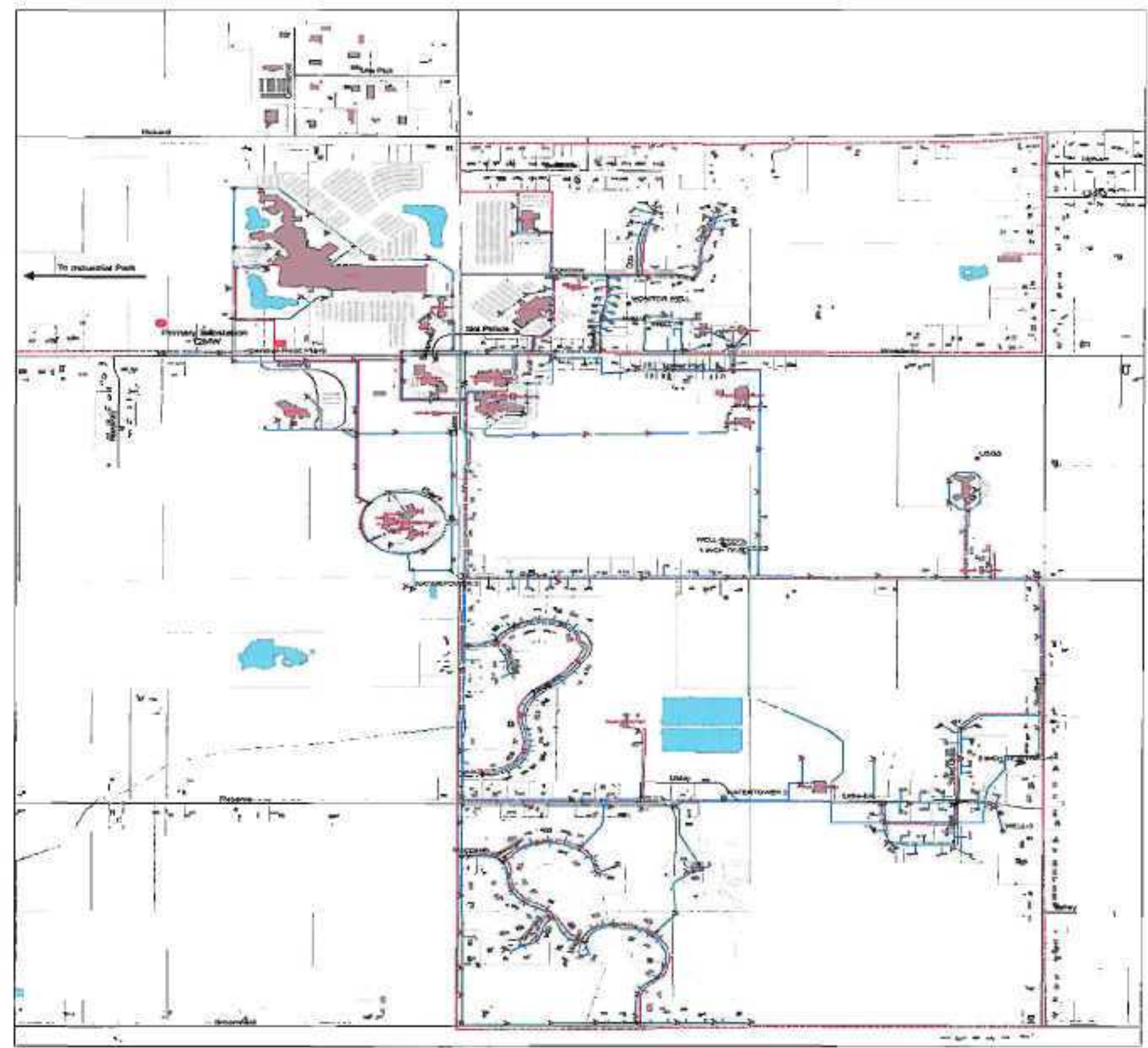

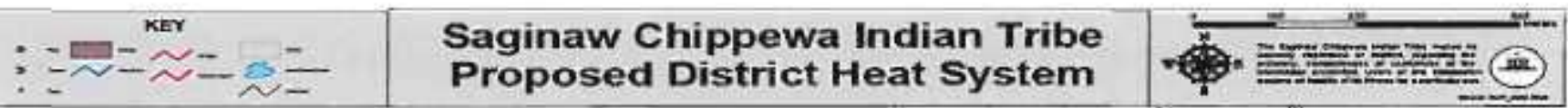


Peak Wood Heat Output (million BTU)

Wood Fuel Cost per ton

Peak Electric Capacity (kW)

Electric CHP Operating Capacity Factor \% Utility Electric Sale Price $\$ / \mathrm{kW}$ - $\mathrm{hr}$ Local Electric Sale Price (to self) \$/kWh

Thermal Heating Capacity Factor \%

Thermal Heating Sales Price $\$ / m m B T U$
$60 \mathrm{mmbtu}$

$\$ \quad 25.00$ /US ton $5,000 \mathrm{~kW}$ $50 \% \mathrm{CF}$

\$ $\quad 0.065 / \mathrm{kW}-\mathrm{hr}$

\$ $\quad 0.065 / \mathrm{kW}$-hr

NA CF

\$ $9.00 \mathrm{mmbtu}$

CAPITAL COSTS

\begin{tabular}{l} 
Wood Fired Unit at Site w/ Boiler \& storage \\
Mechanical Interconnection \& Distribution \\
Steam Turbine \\
Building Retrofit \& Prep \\
Utility interconnection w/transformer \\
Engineering \& Development \\
Legal \& Financial Expense \\
\hline TOTAL CAPITAL COST
\end{tabular}

TOTAL CAPITAL COST

COST SUMMARY ANALYSIS

Installed Capital Cost

First Year Fuel, O\&M \& Admin Cost

First Year Capital Recovery Cost st Year Expense (Debt \& O\&M)

...stalled Cost per KWe

Instailed Cost per kW-hr/yr

First Year Cost per kW-hr w/o REPI

First Yr Cost per kWh w/REPI

\begin{tabular}{l}
$\quad$ First Year Operating Cost Data \\
\hline Fuel \\
Rent \\
Admin \\
O\&M \\
Taxes \\
Insurance \\
Capital Recovery \\
\hline
\end{tabular}

Note: Discount Rato for Present Value Caic.
$\$ 8,000,000$

$\$ 1,270,000$

$\$ 2,000,000$

$\$ 200,000$

$\$ 200,000$

$\$ 80,000$

$\$ 20,000$

$\$ 11,770,000$

$\begin{array}{lrl}\$ 11,770,000 & \\ \$ 924,283 & \\ \$ 823,900 & \\ \$ 1,748,183 & \\ \$ & 2,354 & \\ \$ & 0.537 & \mathrm{lkW}-\mathrm{hr} \\ \$ & 0.015 & \mathrm{lkW}-\mathrm{hr} \\ \$ & (0.003) & \mathrm{kWW}-\mathrm{hr}\end{array}$

$\$ 11,770,000$

$\$ 924,283$

$\$ 823,900$

$\$ 1,748,183$

Energy Efficiency

Total Wood Fuel Energy in mmbtulyr Heat Output mmbtu/year Electric Output kW-hrs/year Electric Output mmbtu/yr

Thermal Efficiency Electric Efficiency Total Efficiency

Energy Cost to Electric kW-hr Price \$

Exess Heat and Electric Sales \$ $\$ 213,120$

262,800

157,680

$21,900,000$

74,745

$60 \%$ $28 \%$ 
60 MMBTU

'.' rod Fired Steam CHP

$17.58 \mathrm{mw}$

Peak Wood Heat Output (million BTU)

Wood Fuel Cost per ton

Peak Electric Capacity (kW)

Electric CHP Operating Capacity Factor \% Utility Electric Sale Price \$/kW-hr Local Electric Sale Price (to self) $\$ / \mathrm{kWh}$

Thermal Heating Capacity Factor \%

Thermal Heating Sales Price \$/mmBTU
$60 \mathrm{mmbtu}$

\$ 25.00 NUS ton $5,000 \mathrm{~kW}$ $50 \% \mathrm{CF}$

$\$ \quad 0.065 / \mathrm{kW}-\mathrm{hr}$

$\$ \quad 0.065 / \mathrm{kW}-\mathrm{hr}$

NA CF

$\$ \quad 9.00 \mathrm{mmbtu}$

\section{CAPITAL COSTS}

Wood Fired Unit at Site $w /$ Boiler \& storage

Mechanical interconnection \& Distribution

Steam Turbine

Building Retrofit \& Prep

Utility Interconnection w/transformer

Engineering \& Development

Legal \& Financial Expense

TOTAL CAPITAL COST
$\$ 8,000,000$

$\$ 9,820,000$

$\$ 2,000,000$

$\$ 200,000$

$\$ 200,000$

$\$ 80,000$

$\$ 20,000$

$\$ 20,320,000$

COST SUMMARY ANALYSIS

Installed Capital Cost

First Year Fuel, O\&M \& Admin Cost

First Year Capital Recovery Cost

st Year Expense (Debt \& O\&M)

...stalied Cost per KWe

Installed Cost per kW-hrlyr

First Year Cost per kW-hr w/o REPI

First $Y r$ Cost per kWh w/REPI
$\$ 20,320,000$

$\$ 940,314$

$\$ 1,422,400$

$\$ 2,362,714$

$\$ 4,064$

$\$ 0.928 / \mathrm{kW}-\mathrm{hr}$

$\$ \quad 0.043 / \mathrm{kW}-\mathrm{hr}$

$\$ \quad 0.025 \mathrm{JW}-\mathrm{hr}$

\section{First Year Operating Cost Data}

\begin{tabular}{l} 
Fuel \\
Rent \\
Admin \\
O\&M \\
Taxes \\
Insurance \\
Capital Recovery \\
\hline
\end{tabular}

Note: Discount Rate for Present Value Calc.

\begin{tabular}{ccr} 
& & Percent \\
\hline$\$$ & 733,914 & $31.1 \%$ \\
$\$$ & - & $0.0 \%$ \\
$\$$ & 43,800 & $1.9 \%$ \\
$\$$ & 124,500 & $5.3 \%$ \\
$\$$ & - & $0.0 \%$ \\
$\$$ & 38,100 & $1.6 \%$ \\
$\$ 1,422,400$ & $60.2 \%$ \\
\hline
\end{tabular}

TOTAL $\$ 2,362,714 \quad 100 \%$ Total O8M \& K Cost less Excess Sale

$3.0 \%$
Present Total Natural Gas Cost/yr

Electric Cost Savings /yr
SECR

100000

Public Facilsties

Residential

Annual Heat Load Requir. (mmbtu)

Heat Output mmbtulyear

Heat Cost per mmbtu \$

Total Heat Fuel Cost/yr \$

Heat Only $\$ / m m b t u$ (w/capital \& O\&M) \$

8.99

Heat Energy \$/mmbtu (fuel only) \$

2.79

N. Gas Cost \$/mmbtu @75\% eff. \$

12.00

Thermal Heat Sales @75\%NG Cost \$ 1,419,120

Total Electric Expense per/yr \$ 943,594

Electric Output kW-hrs/year $\quad 21,900,000$

First Year Electric Cost per kW-hr \$ $\quad 0.043$

Electricity kWh/yr Availabie for Sale $\quad(29,100,000)$

Value of Excess Elec/yr at $\$ .06 / k w h \$(1,746,000)$

Local Consumption Electric kWh

$51,000,000$

Percent Local Electric to Total Gen.

Natural Gas Cost $/$ CCF

$233 \%$

Energy Cost to Electric kW-hr Price \$

1.20

943,594

(assumes thermal energy sold at $75 \%$ NG)

Exess Heat and Electric Sales

$(326,880)$

Total Wood Fuel Energy in mmbtulyr Heat Output mmbtulyear Electric Output kW-hrs/year Electric Output mmbtu/yr

262,800

157,680

$21,900,000$

74,745

Thermal Efficiency

Electric Efficiency

Total Efficiency

$60 \%$

$28 \%$

Net Annual Savings
\$ $2,689,594$

$\$ 1,400,000$

$\$ 479,906$

$(809,688)$ 
Appendix I. Natural Gas Engine Cost and Details 


\section{SECTION 2: CHP TECHNOLOGIES}

\subsection{PRIME MOVERS}

This chapter presents ONLY highlights of the applicable technologies.
For more detailed information, visit the following DOE Website:
www.CHPB.net/prof-status.html

\section{Purpose of Prime Mover:}

Convert fuel energy directly to mechanical shaft power. The shaft power can then drive a generator to produce utility grade electricity. There are many proven prime mover technologies used for generating electricity on-site or near site.

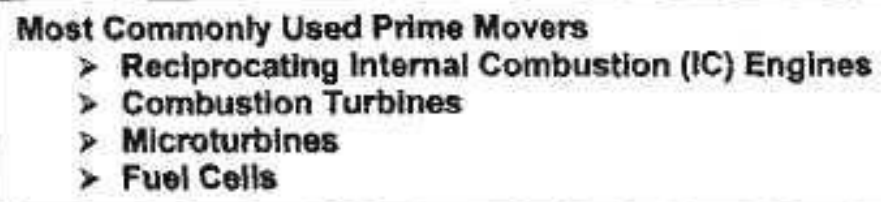

Table 2-1 Prime Mover "Rules-of-Thumb"

\begin{tabular}{|c|c|c|c|}
\hline \multirow{13}{*}{ 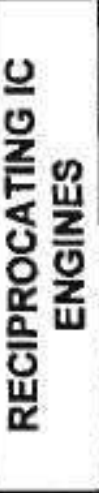 } & Capactly Rage (kW) & $100-500$ & $500-2,000$ \\
\hline & Electric Generation Efficiency & & \\
\hline & \% of LHV of Fuel & $24-28$ & $28-38+$ \\
\hline & Heat Rate, Btu/kWh & $14,000-12,000$ & $12,000-9,000$ \\
\hline & Recoverable Useful Heat & & \\
\hline & Hot Water $\left(9160^{\circ} \mathrm{F}\right)$, Btu/h per kW & $4,000-5,000$ & $4,000-5,000$ \\
\hline & Steam (19 15 psig), Bssh per $\mathrm{kW}$ & $4-5$ & $4-5$ \\
\hline & Installod Cost, $\$ / W W$ & & \\
\hline & (with Heat Racovery) & $1,800-1,400$ & $1,400-1,000$ \\
\hline & O \& M Costs, s/WWh & $0.015-0.012$ & $0.012-0.010$ \\
\hline & $\mathrm{NO}_{x}$ Emission Levels, Hos $\mathrm{MMHH}$ & & \\
\hline & Rich Bum w/3-way catalyst (w/o) & $\sim 0.5(30-40)$ & $-0.5(30-40)$ \\
\hline & Lean Burn w/SCR treatment (w/o) & $-0.5(2-6)$ & $\approx 0.5(2-6)$ \\
\hline \multirow{13}{*}{$\frac{\mathscr{d}}{\frac{w}{2}}$} & Capacity Rage, $\mathrm{kW}$ & $1,000-10,000$ & $10,000-50,000$ \\
\hline & Eloctric Generation Effledency & & \\
\hline & $\%$ of LHV of Fuel & $24-28$ & $31-36$ \\
\hline & Heat Rate, ElunkWh & $14,000-12,000$ & $11,000-8,500$ \\
\hline & Recoverabte Useful Heat & & \\
\hline & Hot Water (ㅇ $150^{\circ} \mathrm{F}$ ), Btuh por $\mathrm{kW}$ & $5,000-8,000$ & $5,000-6,000$ \\
\hline & Steam (@15 psig, Ibsh per iW & $5-6$ & $5-6$ \\
\hline & Installed Cost, S/KW & & \\
\hline & (with Heat Recovery) & $1.500-1.000$ & $1,000-800$ \\
\hline & O \& M Costs, $5 \mathrm{kWM}$ & $0.015-0.012$ & $0.012-0.010$ \\
\hline & NOx Emisaslon Levels, ppm & & \\
\hline & With Dry Low NOx Bumer & $<25$ & $<25$ \\
\hline & With SCR & $<10$ & $<10$ \\
\hline \multirow{11}{*}{ 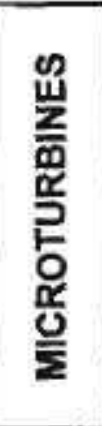 } & Capactiy Rage, $\mathrm{kW}$ & $100-400$ & \\
\hline & Electric Generation Efficiency & & \\
\hline & $\%$ of LHV of Fuel & $25-30$ & \\
\hline & Heat Rate, BtukWh) & $13,700-11,400$ & \\
\hline & Recoverable Usoful Hoat & & \\
\hline & Hot Water (@ 160\%F), Btuh por kW & $6,000-7,000$ & \\
\hline & Sleam (@ 15psig), fbsh per kW & NA & \\
\hline & Installed Cost, $\$ / K W$ & & \\
\hline & (with Heat Recovery) & $2,000-1,000$ & \\
\hline & O\& M Costs, $\$ \mathrm{kWh}$ & $0.015-0.01$ & \\
\hline & NO, Emission Levets, IbsMMm & $<0.49$ & \\
\hline
\end{tabular}




\subsubsection{Reciprocating Internal Combustion Engines (IC Engines)}

One of the most common technologies used for power generation. These engines are the fastest growing segment of the market for CHP systems $<5 M W$.

\section{Sizes:}

Capacities range from about $5 \mathrm{~kW}$ to $10 \mathrm{MW}$.

\section{Characteristics:}

>Better at load following and part load operation than most of the other prime mover technologies.

> Can be fueled by natural gas, diesel or gasoline:

- CHP systems most commonly use natural gas because it resuits in significantly lower emissions than those fueled by the other two fuels.

Most backup and emergency generator sets using IC engines are fueled with diesel or gasoline and are similar to an automotive design. They are generally NOT designed for continuous operation nor are they setup to recover thermal energy from the engine exhaust streams.

- CHP systems generally use industrial grade engines because these are designed for continuous (24/7) operation.

- Two types of ignition systems: spark and compression. Spark ignited engines can use natural gas or gasoline as fuel and compression ignited engines can only use diesel fuel.

> Designed to operate in one of the two modes:

1) Rich-burn operation uses higher fuet-to-air ratios than the stoichiometric ratio (defined as the fuel-to-air ratio theoretically required for complete combustion of the fuel).

- More common for engine capacities $<500 \mathrm{~kW}(670 \mathrm{hp})$.

- Normally produce $\mathrm{NO}_{\mathrm{x}}$ emissions in the range of 30 to $50 \mathrm{lbs}$ per $M W h$ with no exhaust treatment. Therefore, most installations using rich burn engines will REQUIRE a 3-way catalyst to treat the engine exhaust. This can reduce NOx emissions to as low as $0.5 \mathrm{lb} / \mathrm{MWh}$, but adds approximately $\$ 50 / \mathrm{kW}$ to the engine's installed cost.

2) Lean-bum operation uses lower fuel-to-air ratios than the stoichiometric ratio.

- The energy efficiency is slightly higher than that for rich-bum engines.

- Normally produce $\mathrm{NO}_{\mathrm{x}}$ emissions in the range of 2 to $6 \mathrm{lbs}$ per MWh with no exhaust treatment.

- Most installations using lean-burn engines do NOT require exhaust treatment. If exhaust treatment is needed to reduce NOx emissions, the most common treatment is the use of a Selective Catalytic Reduction (SCR). Use of an SCR is very expensive. It adds approximately $\$ 100 / \mathrm{kW}$ to the engine installed cost and $\$ 1400$ /ton of $\mathrm{NO}_{\mathrm{x}}$ removed in operating cost. approximately $3 \mathrm{lbs}$ of NOx per MWh. (Per a-Grid data for the year 2000) 
The fuel utilization efficiency of $1 \mathrm{C}$ engines for producing electricity ranges from approximately $25 \%$ to $40 \%$ on the basis of lower heating value (LHV). Usable thermal energy from these prime movers is normally recovered from two streams: 1) engine exhaust gases, and 2) engine-jacket coolant. Distribution of energy for a typical engine is shown in Figure 2-1.

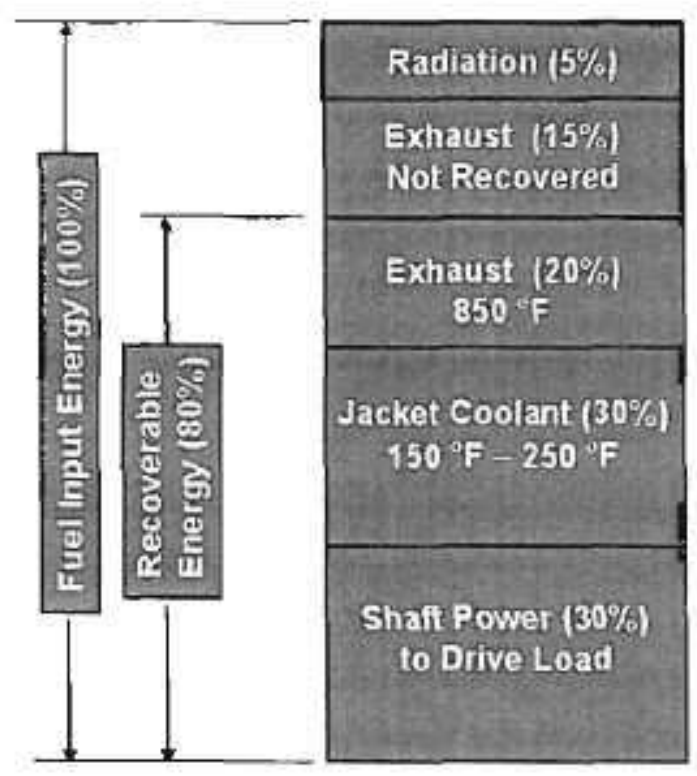

Figure 2-1 Energy Distributions for a Typical Reciprocating Engine 


\subsection{Thermally-Activated Technologies}

The technologies that use thermal energy as the primary energy for their operation are collectively called "Thermally Activated Technologies (TATs)." The three most common TATs that are applicable to CHP systems are as follows:

1. Absorption Chillers

2. Desiccant Dehumidifiers

3. Space and Process Heaters

For a detailed discussion on the benefits of a CHP system, visit the U.S. Department of Energy (DOE) Website (wnw CHPB. net/profbenefit.html) for CHP for Buildings.

\section{Table 2-4 Absorption Chillers "Rules-of-Thumb"}

\begin{tabular}{|c|c|c|c|}
\hline \multirow{7}{*}{ 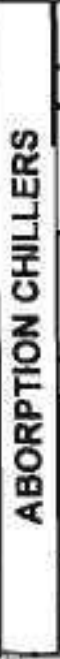 } & Capacity Range (kW) & Single-Effect & $\begin{array}{l}\text { Double- } \\
\text { Effect }\end{array}$ \\
\hline & COP & $0.6-0.67$ & 0.91 .2 \\
\hline & $\begin{array}{l}\text { Heat Source } \\
\text { Minbmum Temperature, }{ }^{\circ} \mathrm{F} \\
\text { Steam Flow Rate, ibsh per RT } \\
\text { Steam Pressure, psig } \\
\end{array}$ & $\begin{array}{l}180 \\
18 \\
15 \\
\end{array}$ & $\begin{array}{c}350 \\
10-11 \\
115-125 \\
\end{array}$ \\
\hline & $\begin{array}{l}\text { Integration w/ Wasto Heat from: } \\
\text { Reciprocating engines, RT/kW } \\
\text { Combustion buibines, RT/kW } \\
\text { Microlubines, RTMW } \\
\end{array}$ & $\begin{array}{l}0.22-0.28 \\
0.28-0.33 \\
0.33-0.45 \\
\end{array}$ & $\begin{array}{l}0.3-0.4 \\
0.4-0.5 \\
0.4-0.5\end{array}$ \\
\hline & Average Electric Power Othet & $0.6 \mathrm{KW} / \mathrm{RT}$ & $0.6 \mathrm{~kW} / \mathrm{RT}$ \\
\hline & $\begin{array}{l}\text { Installed Cost (S/RT) } \\
100 \mathrm{RT} \\
500 \mathrm{RT} \\
1,000 \mathrm{RT} \\
2,000 \mathrm{RT} \\
\end{array}$ & $\begin{array}{c}1000 \\
700 \\
650 \\
500 \\
\end{array}$ & $\begin{array}{c}1200 \\
800 \\
850 \\
700 \\
\end{array}$ \\
\hline & $\begin{array}{l}\text { OsM Costs (SSRT/y) } \\
100 \mathrm{RT} \\
500 \mathrm{RT}-2,000 \mathrm{RT}\end{array}$ & $\begin{array}{c}30 \\
16-28\end{array}$ & $\begin{array}{c}30 \\
17-25\end{array}$ \\
\hline
\end{tabular}

Table 2-5 Desiccant "Rules-of-Thumb"

\begin{tabular}{|c|c|c|c|c|c|c|}
\hline \multirow{7}{*}{$\begin{array}{l}\frac{9}{3} \\
\frac{3}{0} \\
0\end{array}$} & \multirow{2}{*}{$\begin{array}{l}\text { Parameter } \\
\text { Flow Rate }\end{array}$} & \multirow{2}{*}{$\frac{\text { Units }}{\text { SCFM }}$} & \multicolumn{2}{|c|}{ Industrial } & \multicolumn{2}{|c|}{ Commerclal } \\
\hline & & & 600 & 40,000 & 2,000 & 12,000 \\
\hline & Installed Cost & \$SCFM & $\$ 20$ & $\$ 5$ & $\$ 8$ & $\$ 4.50$ \\
\hline & OsiM Costs & E/SCFM/yr & 0.26 & 0.06 & 0.09 & 0.06 \\
\hline & $\begin{array}{l}\text { Regeneration } \\
\left(200^{\circ} \mathrm{F}\right)\end{array}$ & BTUhr per SCFM & 55 & 55 & 45 & 45 \\
\hline & Latent Heat Removal & $\begin{array}{l}\text { lbshit per } 1000 \\
\text { SCFM }\end{array}$ & 35 & 35 & 30 & 30 \\
\hline & Parasitic Electric Use & KWh per 1000 SCFM & 1.1 & 1,1 & 0.8 & 0.8 \\
\hline \multirow{6}{*}{$\frac{\mathrm{Q}}{\mathrm{a}}$} & Flow Rate: & SCFM & 3,000 & 84,000 & 10,000 & 84,000 \\
\hline & Instalied Cost & $\$ /$ SCFM & $\$ 18$ & 55 & $\$ 7$ & $\$ 5$ \\
\hline & O\&M Costs & E/SCFM/Yr & 0.38 & 0.11 & 0.15 & 0.11 \\
\hline & $\begin{array}{l}\text { Regeneration } \\
\left(200^{\circ} \mathrm{F}\right.\end{array}$ & BTU/nr per SCFM & 45 & 45 & 35 & 35 \\
\hline & Latent Heat Removal & $\begin{array}{l}\text { Bos/hr per } 1000 \\
\text { SCFM }\end{array}$ & 30 & 30 & 30 & 30 \\
\hline & Parasitic Electric Use & $\mathrm{KWM}$ per $1000 \mathrm{SCFM}$ & 1.3 & 1.3 & 1.3 & 1.3 \\
\hline
\end{tabular}




\subsubsection{Absorption Chillers}

Absorption chillers are similar to vapor compression chillers with a few key differences.

$>$ Basic difference is that a:

- Vapor compression chiller uses a rotating device (electric motor, engine, combustion turbine or steam turbine) to operate the compressor to raise the pressure of refrigerant vapors, while an

- Absorption chiller uses heat to compress the refrigerant vapors to a high-pressure, therefore this "thermal compressor" has no moving parts.

A process schematic of an absorption chiller is shown in Figure 2-5.

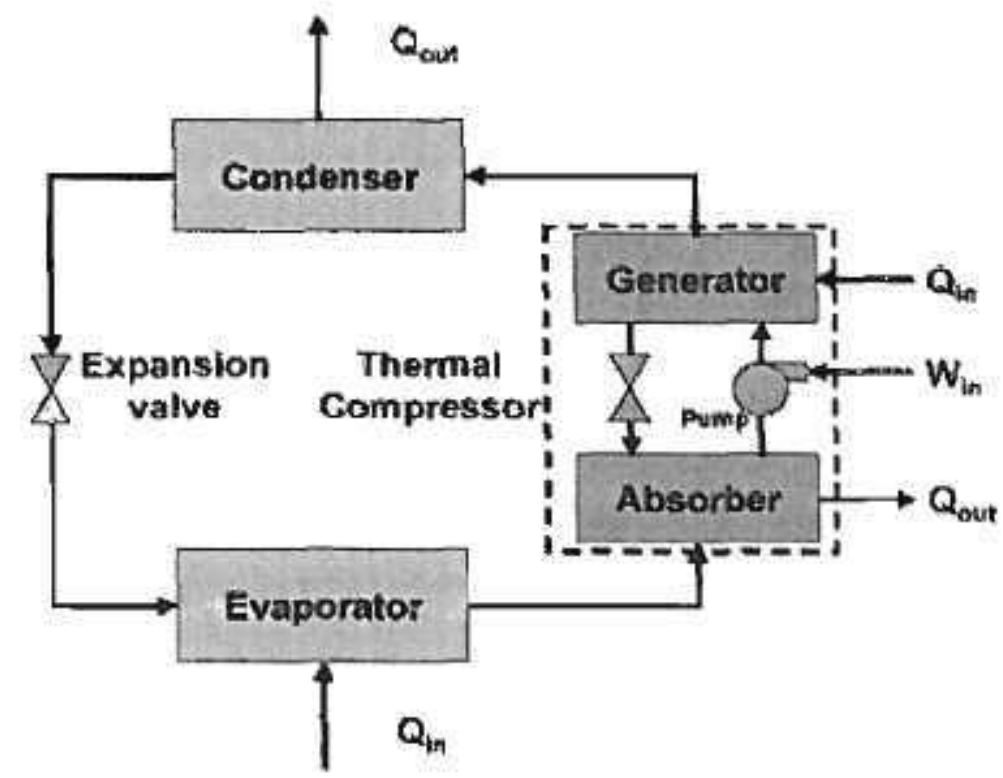

Figure 2-5 Process Schematic of an Absorption Chiller

Commercially available absorption chillers that can be tutilized within a CHP system can use:

1) Steam,

2) Hot Water, or

3) Hot Exhaust Gases

> Current absorber media / refrigerant for absorptions chillers are either.

- Lithium bromide / water, or

- Ammonia / water

Two types of steam absorption chillers are commercially available:

1. Single Effect:

- Lower initial costs but less efficient and thus take more energy and are more expensive to operate.

- Requires about $18 \mathrm{lbs} / \mathrm{h}$ of steam at $15 \mathrm{psig}$ for 1 ton of cooling.

- Most CHP systems utilize single effect absorption chillers to keep initial cost low. 
2. Double Effect:

- Higher initial costs but are more energy efficient and thus require less energy to operate.

- Requires about $10 \mathrm{lbs} / \mathrm{h}$ of steam at about $120 \mathrm{psig}$ for 1 ton of cooling.

> Rated capacities of absorption chillers are based on producing chilled water at $44^{\circ} \mathrm{F}$.

- Absorption chillers can also be used in chilled water storage systems to produce chilled water during off-peak electric load periods when the cost of electricity is low and the demand for cooling is low. The stored chilled water is then drawn upon during the peak cooling periods when electricity costs are high, to supplement the chiller operation. The storage system heips to reduce the chiller capacity requirement and total installed cost of chillers. 


\section{SECTION 4: FEASIBILITY EVALUATION}

Implementing a CHP system requires significant time, effort, and investment. Therefore, it's prudent to first estimate its financial AND technical feasibility using a systernatic approach that incorporates the sequence of the process outlined below. The three levels of analyses have different scope, depth of analysis and accuracy of total costs to complete, and require different levels of effort.

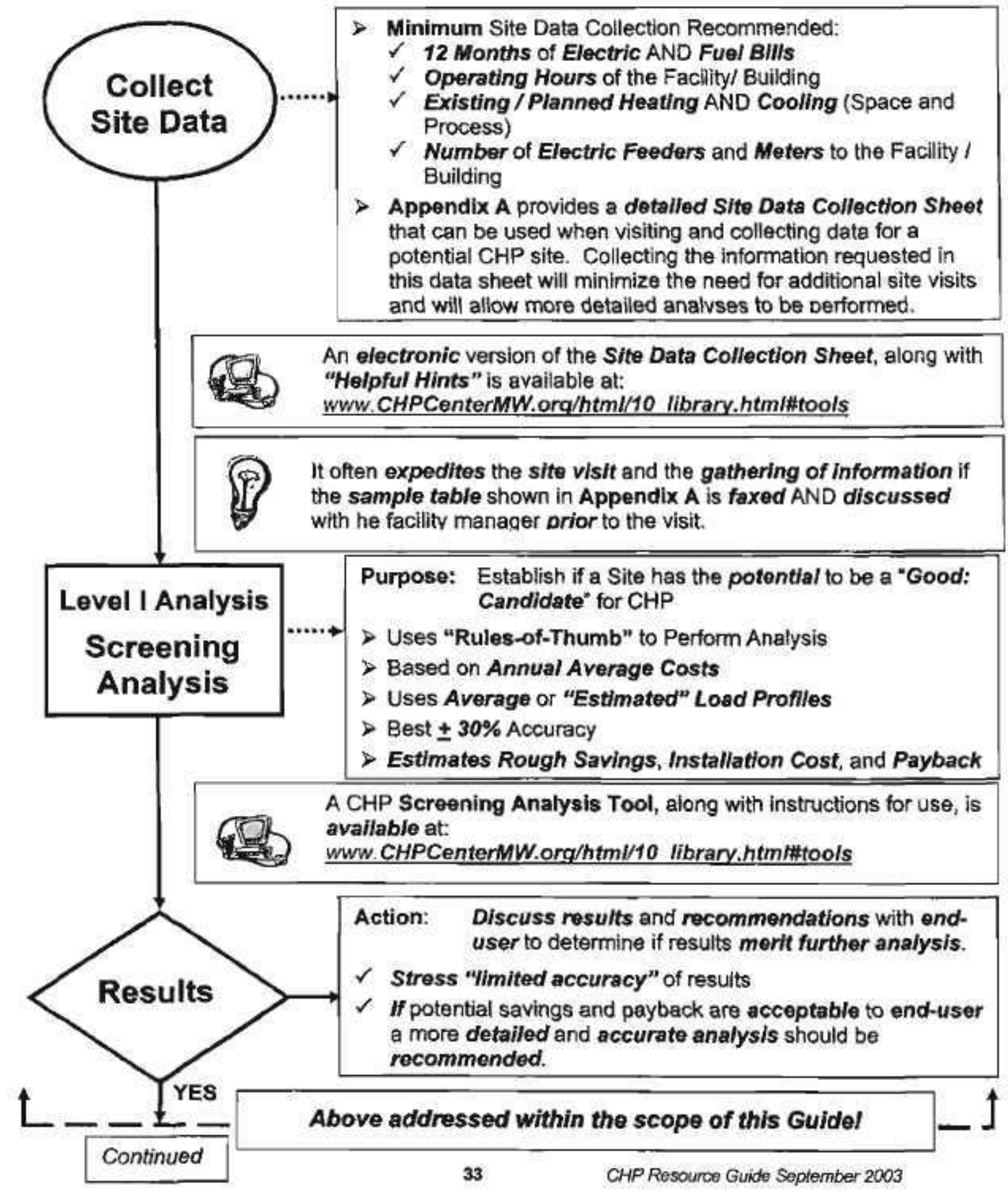




\section{TLAME}

Technology You Can Trust

Absorption water chillers and Trane have been synonymous for forty years. In fact, Trane is the only North American chiller manufacturer to commercialize double effect absorption over 25 years ago. With over 10,000 absorption chillers manufactured and shipped, Trane serves the commercial, industrial and process worldwide markets. Trane continues its worldwide leadership as the number one provider in absorption, centrifugal, scroll and helical rotary chillers.

\section{Dynamic By Design}

In the early 1990's, with the assistance of the Gas Research institute, Trane began development of a series of actvanced absorption chiller designs. The new design was to redefine industry standards for overall system performance, efficiency and reliability.

In 1996. Trane announced the Horizon" line of two-stage steamhot water absorption chillers. True to its name and true to the high standardis for its design, the Trane Horizon chiller offers system advantages that go beyond those of other absorption chillers currently on the market.

\section{Operates With Stearn $\leq 13$ psig} or Hot Water $\leq 270 \mathrm{~F}$

in 2000, the Horizon series expanded to include single stage hot water and stearn fired absorption. This state-ofthe-art chilier design can produce chilled water temperatures as coid as 40 Fand start up with tower water temperatures as low as $55 F$ These lower operating temperatures can significantly reduce system energy consumption and cost.

This capability of making chilled water from these comparatively low temperature inputs is particulariy important for energy conserving applications such as waste heat recovery, co-generation equipment and solar-energy powered cooling. Using water as the refrigerant helps eliminate concerns about refrigerant management and availability. Additionally, atsomption technology reduces the requirement for electric energy.

\section{Trane Horizon ${ }^{\mathrm{Tm}}$ Absorption Series Single-Stage Hot Water or Steam-Fired Absorption Water Chillers, 500-1350 Tons}
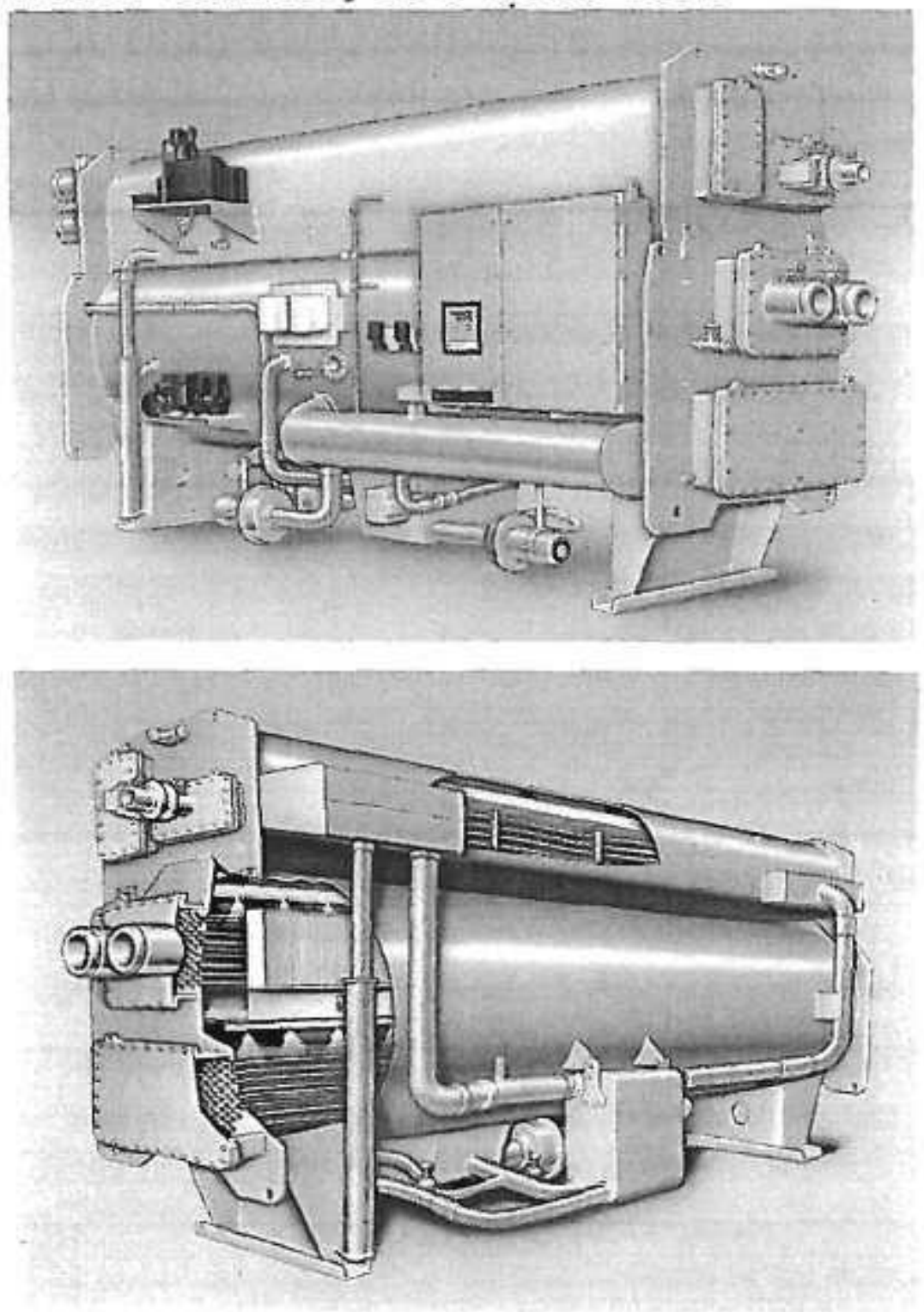

Sophisticated Reliabisity

Whether your specification calls for standalone chiller control, hybrid chiller control, or a building automation system, the Trane UCP2 control panel as provided on all Horizon chiliers, is critical to reliable operation and optimal performance. Controls from Trane are compatible with Integrated Comfort" systems (ICS) and thoy can be easily imtegrated into the Tracer " family of flexible chilier plant system controllers with a single twisted-wire pair communications cable. 
Ideal for Process and Commercial Applications

With Horizon chillers, the application possibilities for the absorption machine are expanded. Capabilities such, 85 lower tower flow, variabie evaporator flow, lower chilled water temperatures and advanced control capabiltíes make the single-stage Horizon absorption chiller ideal for both process and comfort applications.

\section{When Long-lived Reliability} is Important

Trane has been a long-time proponent of the use of high quality materiais in absorption chiller designs. The lithium bromide temperatures and water refrigerant, typical of all absorbers, can more quickly corrode lower-grade metais in the presence of air. Trane recommends and uses industrialgrade materials to provide long-lived, reliable cooling.

\section{A Global Network of Absorption Expertise} When you specify a Trane Horizon chiller, you're getting the knowledge, expertise and assistance of a pool of experts with decades of absorption expertise. Making The Trane Company part of your management team gives you access to refrigeration, air conditioning and facility control system applications specialists and a unique breadth of innovative solutions to satisfy your facilities needs for today... and tomorrow.

\section{Standard Specification For}

Single-Stage Horizon Chillers

- C.O.P. 0.70

- Victaulic" water connections

- Industrial-grade tubes

- Generator .028 wall, 90/10

Cupro-nickel

- Evaporator .025 copper

- Absorber $500-800$ tons .022" wall 95/5 Cupro-nickel. 975-1350 tons .028" wail copper

- Condenser $0.028^{\prime \prime}$ wall copper

- 150 psig pressure design on evaporator, absorber and condenser sections

- Industrial grade energy valve

- Rigging eyes for easy installation

\section{The Trane Compary \\ An Americen Standiard Company unmw, trane.com}

Formore information contact your local district office or e-mail us at comfore@trane.com

QAnterion Sundard has 2001

Horizon" Single-Stage Steam-Fired Absorption Unit

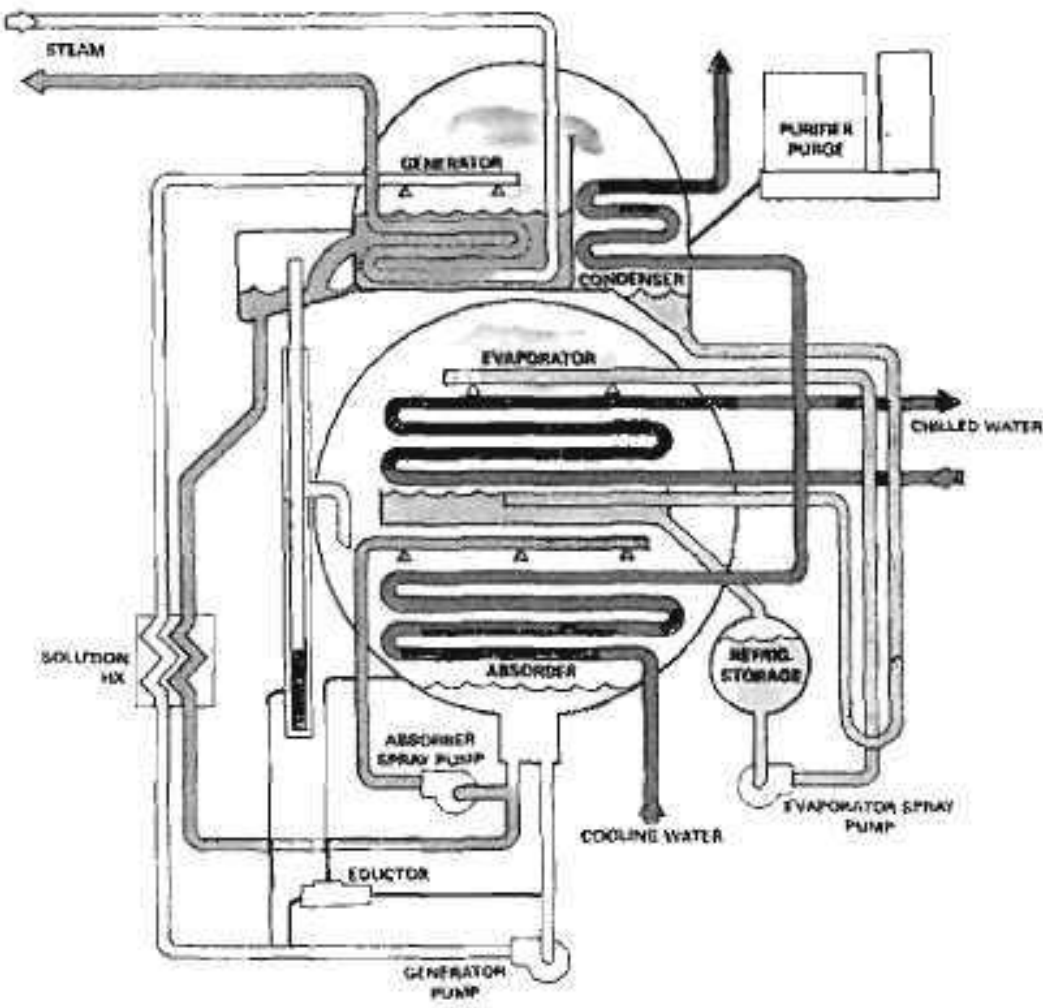

- Advanced microprocessor control system with adaptive control functions

- 2-line, 40 character clear language interface to unit functions and diagnostic information

- Fixed and floating generator tube supports help prevent thermal stress

- Efficient stainless steel brazed plate solution heat exchangers

- Long life solution pumps

- Molybdate inhibitor system

- Factory installed and commissioned controls

- Ali tubes individually replaceabie

- Rernovable absorber and evaporator spray tree systems

Optional Specification For Single-Stage Horizon Chillers

- Advanced cycle management system with Adaptive Frequency ${ }^{2}$ drive solution control

- 150 psig raised face fianges for the evaporator, condenser, and absorber water connections

Slace The Trane Company has a polky of concinuous produca and prothes deta inprovernerh, hireserves the rogtrt to change desigh and apecification without notice.
- Oisassembled unit - providing for easy disassembly and reassembly of major components at the job site

- Lithium bromide filter

- Marine style waterboxes on the condenser and absorber sections

- Cooling water crossover pipe factory instalied between the absorber and condenser

- Factory mounted energy vatve

- Fully automatic purge system

- Chaice of tube materials and options

\section{Absorption Cooling -}

A Sound Decision

Life cycle costing has become a primary concem for chiller buyers wtio have long-term investment opportunity in mind. Changes in the distribution and pricing of electricity have made the absorption water chiller a popular choice when alternative energy use makes sense. Ask your iocal Trane representative for a comprehensive analysis of your facility and the energy saving opportunities Trane offers for the dssign of HVAC systems and controls. 


\section{JMS Natural Gas Budget Pricing}

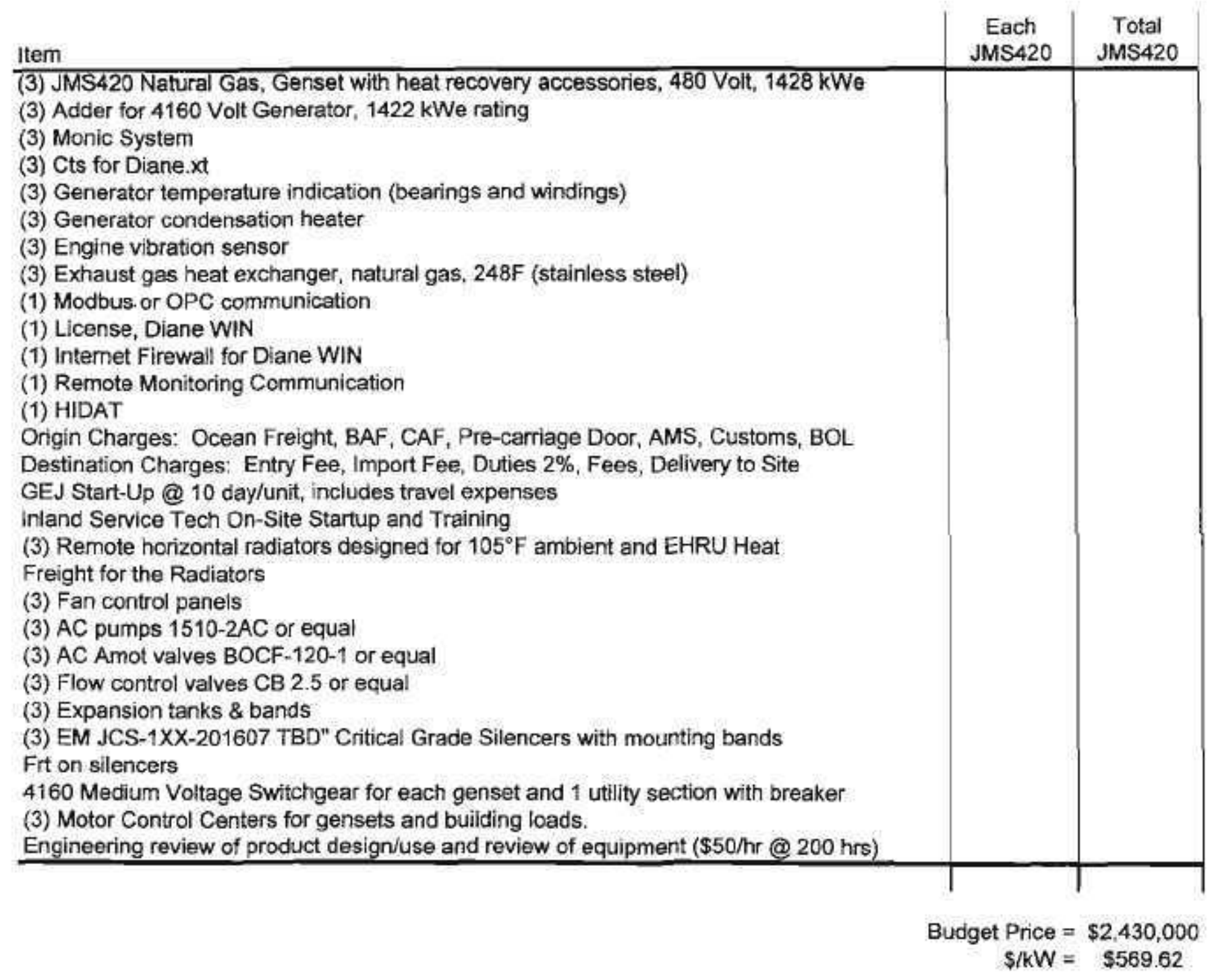


Captial Cost Estimates for Energy Plant with 3 - JMS420 Units and Heat Recovery

$\$ 100,000$ Engineering costs

$\$ 70,000$ Civil (equipment pads, trenching of pipes and electrical)

$\$ 80,000$ Concrete (equipment pads)

$\$ 225,000$ Mechanical (building, gas, oil and water pipes, equipment installation and insulation)

$\$ 180,000$ Electrical (equipment installation)

$\$ 20,000$ Crane (equipment installation)

$\$ 2,430,000$ (3) JMS420, $1422 \mathrm{kWe}$ each $(4,266 \mathrm{kWe}$ total) with heat recovery and switchgear

$\$ 3,105,000$ or $\$ 727.85 / \mathrm{kWe}$ 


\title{
Definitions of Services
}

\author{
Preventative Maintenance Contract \\ Includes: \\ Preventative, regular maintenance according to maintenance schedule. \\ Consumables which are needed for maintenance according to the maintenance \\ schedule, inciuding spark plugs, original Jenbacher exchange cylinder heads, etc. \\ Minor overhauls per the maintenance schedule. \\ Major overhaul is not included and should be accrued for separately by customer. \\ Not Included \\ Labor provided by Inland Power Group \\ Possible repairs which may be required outside the maintenance schedule. \\ inspection, checking and operating activities. \\ Supply of lubricating oil, disposal of waste oil \\ Operating commodities
}

\begin{abstract}
Corrective Maintenance Budget
Includes:

Labor provided by Inland Power Group.

Not Included:

Preventative Maintenance scope.

Inspection, checking and operating activities.

Supply of lubricating oil, disposal of waste oil

Operating commodities
\end{abstract}

Possible repairs which may be required outside the maintenance schedule.

\section{Lubricating Ofl Budget - Customer Provided}

Includes:

Price for lubricating oil for the operations of the engine generators.

Estimated an engine oil change at every $1000 \mathrm{oph}$.

Not Included.

Price for oil sampling every 500 oph.

Oil filters, and rings. Included with Preventative Maintenance.

Disposal of waste oil.

Labor for oil change services

\section{Operations Budget - NOT PROVIDED}

Includes:

Price for an operator working part-time on generator sets.

Operation of the plant in compliance with legal and local regulations.

Inspection, checking and operating activities

Acquisition of operating data

Keeping operating logs

Visual checking of the plant

Ignition voltage checking

Trouble shooting

Documentation on the availability of the plant

Thorough documentation of all servicing and maintenance measures

Operating commodities

Not Included:

Tools other than the standard toois provided by GE Jenbacher

Preventative Maintenance scope.

Corrective Maintenance scope.

Supply of lubricating oil, disposal of waste oil 
GE Jenbacher - 3 JMS420 Genset Modules

Producing $1422 \mathrm{kWe}$ each at $4160 \mathrm{~V}$ on Natural Gas

\begin{tabular}{|c|c|c|c|c|c|}
\hline & \multirow[b]{2}{*}{ Responsibility } & \multicolumn{2}{|c|}{2008 Pricing } & \multirow{2}{*}{$\begin{array}{c}3 \text { Units } \\
8400 \text { hrs/year } \\
\text { Year }\end{array}$} & \multirow{2}{*}{$\begin{array}{c}59,999 \text { oph } \\
-7 \text { Years } \\
\text { Contract }\end{array}$} \\
\hline & & \$/oph & \$/kWh & & \\
\hline Preventive Maintenance & Inland & $\$ 7.56$ & $\$ 0.00532$ & $\$ 190,512$ & $\$ 1,360,777$ \\
\hline Corrective Maintenance & Estimate (budget) & $\$ 2.66$ & $\$ 0.00187$ & $\$ 67,032$ & $\$ 478,792$ \\
\hline Operations & Customer (budget) & $\$ 2.00$ & $\$ 0.00141$ & $\$ 50,400$ & $\$ 359,994$ \\
\hline Lubricating Oil & Customer (budget) & $\$ 1.78$ & $\$ 0.00125$ & $\$ 44,856$ & $\$ 320,395$ \\
\hline & & $\$ 14.00$ & $\$ 0.00985$ & $\$ 352,800$ & $\$ 2,519,958$ \\
\hline
\end{tabular}

NOTES:

1. Preventive Maintenance terms would be for 59,999 oph, customer responsible for major overhaul accrual,

2. Estimated 2008 cost of a JMS420 major overhaul $=\$ 240,000$

3. Estimates are based upon A05 Maintenance Schedule for Natural Gas.

4. Operations, provided by Customer, should include estimates for one operator to work 10-20 hours/week.

5. Operations, provided by Customer, shall provide oil changes and daily operations.

6. Gas skid PM parts/service is not included, no gas treatment $\mathrm{H} 2 \mathrm{~S}$ or Siloxane media is included above.

7. Assumes you meet GE Jenbacher gas guidelines (for example, below $\sim 250 \mathrm{ppmv}$ for $\mathrm{H} 2 \mathrm{~S}$ on average)

8. Assumes price of oil at $\$ 10 /$ gallon and oil changes at 2000 operating hours. 
Technical Description

Cogeneration Unit

JMS 420 GS-N.L

J 420

Electrical output

Thermal output

\section{Emission values}

NOx $<0.6 \mathrm{~g} / \mathrm{bhp} . \mathrm{hr}$ (NO2)
$1426 \mathrm{~kW}$ el.

5695 MBTU/hr 
0.01 Technical Data (at module) 3

Main dimensions and weights (at module)

0.02 Technical data of engine

Thermal energy balance

Exhaust gas data

Combustion air data

Output / fuel consumption

Sound pressure level

Sound power level

0.03 Technical data of generator

Reactance and time constants

0.04 Technical data of heat recovery 8

General data - Hot water circuit $\quad 8$

$\begin{array}{ll}\text { Mixture Intercooler (1st stage) } & 8\end{array}$

$\begin{array}{ll}\text { Mixture Intercooler (2nd stage) (Intercooler separate) } & 8\end{array}$

$\begin{array}{ll}\text { Heat exchanger lube oil } & 8\end{array}$

$\begin{array}{ll}\text { Heat exchanger engine jacket water } & 8\end{array}$

$\begin{array}{lr}\text { Exhaust gas heat exchanger } & 8\end{array}$

connection variant $F[9$

0.10 Technical parameters __ 10 


\subsection{Technical Data (at module)}

Data at:

\begin{tabular}{|c|c|c|c|c|c|c|}
\hline Fuel gas LHV & & BTUisct & & 868 & & \\
\hline & & & & $100 \%$ & $75 \%$ & $50 \%$ \\
\hline Energy input & & MBTU/hr & [2] & 12,220 & 9,474 & 6,728 \\
\hline Gas volume & & seffhe & $y$ & 14,078 & 10,915 & 7,751 \\
\hline Mechanical output & & bhp & [1] & 1,966 & 1,475 & 983 \\
\hline Electrical output & & kw el. & [4] & 1,426 & 1,068 & 706 \\
\hline \multicolumn{7}{|l|}{ Recoverable thermal output } \\
\hline$\sim$ Intercooler 1st stage & & MBTU/hr & & 716 & & \\
\hline$\sim$ Lube oil & & MBTUMins & & 448 & & \\
\hline Jacket water & & MBTLims & & 1,637 & & \\
\hline$\sim$ Exhaust gas cooled to $248^{\circ} \mathrm{F}$ & & MBTU/hr & & 2,894 & & \\
\hline Total recoverable thermal output & & MBTU/M & [5] & 5,695 & & \\
\hline & & & & & & \\
\hline \multicolumn{7}{|l|}{ Heat to be dissipated } \\
\hline$\sim$ Intercooler 2nd stage & & MBTU/hr & & 345 & & \\
\hline$\sim$ Lube oil & & MBTU/ht & & $\sim$ & & \\
\hline$\sim$ Surface heat & ca. & METU/hr & D & 394 & & \\
\hline$\sim$ Balance heat & & MBTU/hr & & 122 & & \\
\hline Spec. fuel consumption of engine & & BTU/bhp.hr & {$[2]$} & 6,216 & & \\
\hline Lube oil consumption & ca. & galhr & [13] & 0.14 & & \\
\hline Electrical efficiency & & $\%$ & & $39.8 \%$ & & \\
\hline Thermal efficiency & & $\%$ & & $46.6 \%$ & & \\
\hline Total efficiency & & $\%$ & [6] & $86.4 \%$ & & \\
\hline \multicolumn{7}{|l|}{ Hot water circuit: } \\
\hline Forward temperature & & ${ }^{\circ} \mathrm{F}$ & & 194.0 & & \\
\hline Return temperature & & ${ }^{\circ} \mathrm{F}$ & & 158.0 & & \\
\hline Hot water flow rate & & GPM & & 316.4 & & \\
\hline
\end{tabular}

) approximate value for pipework dimensioning

L. Explanations: see 0,10-Technical parameters

All heat data is based on standard conditions according to attachment 0.10 . Deviations from the standard conditions can result in a change of values within the heat balance, and must be taken into consideration in the layout of the cooling circuit/equipment (intercooler, emergency cooling, ...). In the specifications in addition to the general tolerance of $+l-8 \%$ on the thermal output a further reserve of $10 \%$ is recormmended for the dimensioning of the cooling requirements. 
Main dimensions and weights (at module)

\begin{tabular}{l|c|c}
\hline Length & in & -280 \\
\hline Width & in & $\sim 80$ \\
\hline Height & in & $\sim 90$ \\
\hline Weight empty & ibs & $\sim 33,040$ \\
\hline Weight filled & lbs & $\sim 34,590$ \\
\hline
\end{tabular}

\section{Connections}

\begin{tabular}{|c|c|c|}
\hline Hot water inlet and outlet & in/los & $4 " 1 / 145$ \\
\hline Exhaust gas outlet & in/los & $12 " / 145$ \\
\hline Fuel gas (at gas train) & in/los & $3^{\prime \prime} / 232$ \\
\hline Fuel Gas (at module) & in/lbs & $5^{\prime \prime} / 145$ \\
\hline Water drain ISO 228 & G & $1 / 2 "$ \\
\hline Condensate drain & in/lbs & $2 " 1145$ \\
\hline Safety valve - jacket water ISO 228 & in/os & $2 \times 13 / 2^{\prime \prime} / 2.5$ \\
\hline Safety valve - hot water & in/los & $21 / 21 / 232$ \\
\hline Lube oil replenishing (pipe) & in & 1.1 \\
\hline Lube oil drain (pipe) & in & 1.1 \\
\hline Jacket water - filling (flex pipe) & in & 0.5 \\
\hline Intercooler water-Inlet/Outlet 1st stage & in/lbs & $4 " / 145$ \\
\hline Intercooler water-InletOutlet 2nd stage & in/tibs & $21 / 211 / 145$ \\
\hline
\end{tabular}




\subsection{Technical data of engine}

\begin{tabular}{|c|c|c|}
\hline Manufacturer & & GE Jenbacher \\
\hline Engine type & & J 420 GS-A 86 \\
\hline Working principle & & 4-Stroke \\
\hline Configuration & & $\vee 70^{\circ}$ \\
\hline No. of cylinders & & 20 \\
\hline Bore & in & 5.71 \\
\hline Stroke & in & 7.28 \\
\hline Piston displacement & cu in & 3,728 \\
\hline Nominal speed & $\mathrm{rmm}$ & 1,800 \\
\hline Mean piston speed & $\mathrm{in} / \mathrm{s}$ & 437 \\
\hline Filling capacity lube oil & gal & 121 \\
\hline Filing capacity water & gal & 61 \\
\hline Length & in & 148 \\
\hline Width & in & 62 \\
\hline Height & in & 80 \\
\hline Weight dry & las & 14,551 \\
\hline Weight filled & Ibs & 16,094 \\
\hline Moment of inertia & $b s-f^{2}$ & 276.26 \\
\hline Direction of rotation (from flywheel view) & & left \\
\hline Flywheel connection & & SAE $18^{\prime \prime}$ \\
\hline Radio interference level to VDE 0875 & & $\mathrm{~N}$ \\
\hline Starter motor output & $k W$ & 9 \\
\hline Starter motor voltage & $\mathrm{v}$ & 24 \\
\hline \multicolumn{3}{|l|}{ Thermal energy balance } \\
\hline Enerav input: & MBTU/hr & 12.220 \\
\hline Intercooler & MBTU/hr & 1,061 \\
\hline Lube oil & MBTU/hr & 448 \\
\hline Jacket water & MBTUAhr & 1,637 \\
\hline Exhaust gas total & MBTUMr. & 3,688 \\
\hline Exhaust gas cooled to $356^{\circ} \mathrm{F}$ & MBTU/hr & 2,386 \\
\hline Exhaust gas cooled to $212^{\circ} \mathrm{F}$ & MBTU/hr & 3,063 \\
\hline Surface heat & MBTU/hr & 259 \\
\hline Balance heat & MBTU/hr & 122 \\
\hline \multicolumn{3}{|l|}{ Exhaust gas data } \\
\hline Exhaust gas temperature at full load & ${ }^{\circ} \mathrm{F} \quad[8]$ & 844 \\
\hline Exhaust gas mass flow rate, wet & losithr & 18,183 \\
\hline Exhaust gas mass flow rate, dry & bs $/ \mathrm{hr}$ & 16,907 \\
\hline Exhaust gas volume, wet & scfirr & 229,980 \\
\hline Exhaust gas volume, dry & sctmi & 205,440 \\
\hline Max.admissible exhaust back pressure after engine & psi & 0.870 \\
\hline \multicolumn{3}{|l|}{ Combustion air data } \\
\hline Combustion air mass flow rate & $\mathrm{bs} / \mathrm{hr}$ & 17.615 \\
\hline Combustion air volume & SCFM & 3,637 \\
\hline Max admissible pressure drop in front of intake-air filter & psi & 0.145 \\
\hline
\end{tabular}

\section{base for exhaust gas data: natural gas: $100 \% \mathrm{CH} 4$; biogas $65 \% \mathrm{CH} 4,35 \% \mathrm{CO} 2$}


Output / fuel consumption

\begin{tabular}{l|c|c}
\hline ISO standard fuel stop power ICFN & php & 1,966 \\
\hline Mean effe. press. at stand. power and nom. speed & psi & 232 \\
\hline Fuel gas type & & Natural gas \\
\hline Based on methane number & MN d) & 70 \\
\hline Compression ratio & Epsilon & 12.50 \\
\hline Min./Max fuel gas pressure at inlet to gas train & & \\
\hline Allowed Fluctuation of fuel gas pressure & psi & $1.8-2.9 \mathrm{c})$ \\
\hline Max. rate of gas pressure fluctuation & $\%$ & \pm 10 \\
\hline Maximum Intercooler 2nd staqe inlet water temperature & psisec & 0.145 \\
\hline Spec. fuel consumption of engine & ${ }^{\circ} \mathrm{F}$ & 122 \\
\hline Specific lube oil consumption & BTu/ohp.hr & 6,216 \\
\hline Max. Oil temperature & qibhp.hr & 0.22 \\
\hline Jacket-water temperature max. & ${ }^{\circ} \mathrm{F}$ & 185 \\
\hline
\end{tabular}

c) Lower gas pressures upon inquiry

d) based on methane number caiculation software AVL 3.1

\section{Sound pressure level}

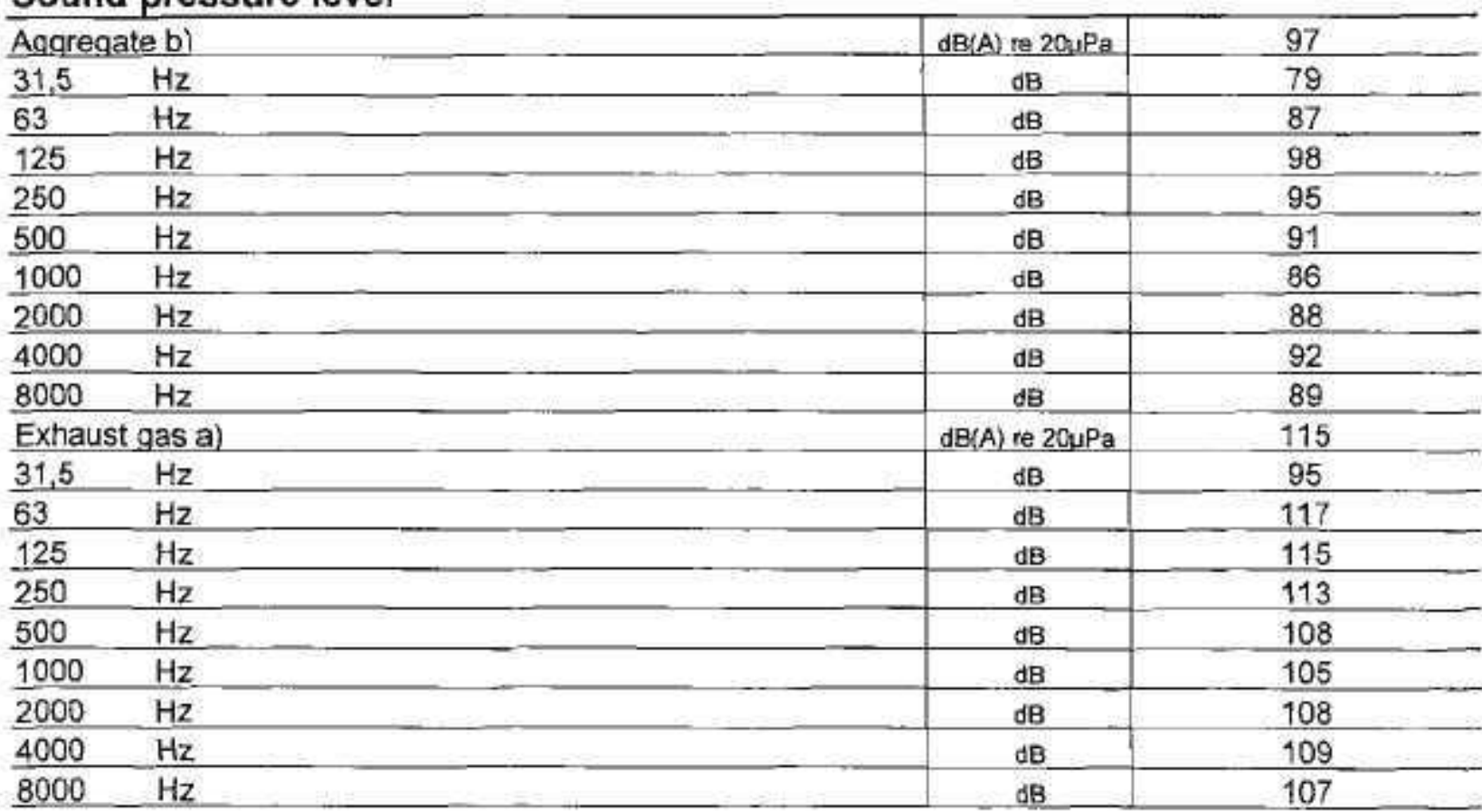

\section{Sound power level}

\begin{tabular}{l|c|c}
\hline Agaregate & $\mathrm{dB}(\mathrm{A}) \mathrm{re} 1 \mathrm{pW}$ & 117 \\
\hline Measurement surface & $\mathrm{ft}^{2}$ & 1.152 \\
\hline Exhaust gas & $\mathrm{dB}$ (A) $\mathrm{re}$ 1pW & 123 \\
\hline Measurement surface & $\mathrm{ft}^{2}$ & 67.60 \\
\hline
\end{tabular}

a) average sound pressure level on measurement surface in a distance of 3.28 according to DIN 45635 , precision class 2 .

b) average sound pressure level on measurement surfoce in a distance of 3.28 th (converted to free fielo) according to DIN 45635 , precision class 3 .

Operation with $1200 \mathrm{mpm}$ see upper values, operation with $1800 \mathrm{rpm}$ add $3 \mathrm{~dB}$ to upper values.

Engine tolerance $\pm 3 \mathrm{~dB}$ 


\subsection{Technical data of generator}

\begin{tabular}{|c|c|c|}
\hline Manufacturer & & STAMFORD E) \\
\hline Type & & PE 734 E2 e) \\
\hline Type rating & KVA & 2,300 \\
\hline Driving power & bhp & 1,966 \\
\hline Ratings at $p . f=1.0$ & $k W$ & 1,426 \\
\hline Ratings at $p . f=0.8$ & $\mathrm{~kW}$ & 1,416 \\
\hline Rated output at p.f. $=0.8$ & kVA & 1,770 \\
\hline Rated current at p.f. $=0.8$ & A & 2,129 \\
\hline Frequency & $\mathrm{Hz}$ & 60 \\
\hline Voltage & v & 480 \\
\hline Speed & $\mathrm{rpm}$ & 1,800 \\
\hline Permissible overspeed & $\mathrm{rpm}$ & 2,160 \\
\hline Power factor lagging & & $0,8-1,0$ \\
\hline Efficiency at $p . f=1.0$ & $\%$ & $97.3 \%$ \\
\hline Efficiency at p.f. $=0.8$ & $\%$ & $96.6 \%$ \\
\hline Moment of inertia & bs-ft $t^{2}$ & 1055.93 \\
\hline Mass & lbs & 7,729 \\
\hline Radio interference level to VDE 0875 & & $\mathrm{~N}$ \\
\hline Construction & & B3/B14 \\
\hline Protection Class & & IP 23 \\
\hline Insulation class & & $\mathrm{H}$ \\
\hline Temperature rise (at driving power) & & $\mathrm{F}$ \\
\hline Maximum ambient temperature & ${ }^{\circ} \mathrm{F}$ & 104 \\
\hline Total harmonic distortion & $\%$ & 1.5 \\
\hline
\end{tabular}

\section{Reactance and time constants}

\begin{tabular}{|c|c|c|}
\hline$\overline{x d}$ direct axis synchronous reactance & p.u, & 2.47 \\
\hline$x d^{\prime}$ direct axis transient reactance & p.u. & 0.15 \\
\hline$x \mathrm{~d}^{\prime \prime}$ direct axis sub transient reactance & p.u. & 0.11 \\
\hline Id" sub transient reactance time constant & $\mathrm{ms}$ & 20 \\
\hline Ta Time constant direct-current & ms & 20 \\
\hline Tdo' open circuit field time constant & s & 2.46 \\
\hline
\end{tabular}

e) GE Jenbacher reserves the right to change the generator supplier and the generator hype. The contractual data of the generator may thereby change slightly. The contractual produced electrical power witl not change. 


\subsection{Technical data of heat recovery}

\section{General data - Hot water circuit}

Total recoverable thermal output

Return temperature

Forward temperature

Hot water flow rate

Design pressure of hot water

Pressure drop hot water circuit

Maximum Variation in return temperature

Max. rate of retum temperature fluctuation

\begin{tabular}{|c|c|c|}
\hline 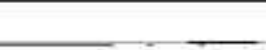 & MBTU/hr & 5,695 \\
\hline & ${ }^{\circ} \mathrm{F}$ & 158.0 \\
\hline & ${ }^{\circ} \mathrm{F}$ & 1940 \\
\hline & GPM & 316.4 \\
\hline & psi & 145 \\
\hline & psi & 13.05 \\
\hline e & ${ }^{\circ} \mathrm{F}$ & $+01-36$ \\
\hline on & ${ }^{\circ} \mathrm{F}$ /min & 18 \\
\hline
\end{tabular}

\section{Mixture Intercooler (1st stage)}

Type

Design pressure of hot water

Pressure drop hot water circuit

Hot water connection

\begin{tabular}{l|c|c} 
& \multicolumn{2}{|c}{ qilled pipes } \\
\hline psi & 145 \\
\hline & psi & 4.35 \\
\hline & in/lbs & $4^{\prime \prime \prime} / 145$ \\
\hline
\end{tabular}

\section{Mixture intercooler (2nd stage) (Intercooler separate)}

Trpe

Design pressure of hot water

Pressure drop hot water circuit

Hot water connection

\begin{tabular}{|c|c}
\hline & qilled pipes \\
\hline psi & $\frac{145}{10.15}$ \\
\hline psi & $\frac{1 \%}{21 / 2 / 145}$ \\
\hline in/bs & \\
\hline
\end{tabular}

\section{Heat exchanger lube oil}

Type

Design pressure of hot water

Pressure drop hot water circuit

Hot water connection

\begin{tabular}{c|c|c}
\hline & \multicolumn{2}{|c}{ plate heat exchanger } \\
\hline & psi & 145 \\
\hline & psi & 2.90 \\
\hline & in/libs & $4^{\prime \prime} / 145$ \\
\hline
\end{tabular}

Heat exchanger engine jacket water

Trpe

Design pressure of hot water

Pressure drop hot water circuit

Hot water connection

plate heat exchanqer

145

2.90

\begin{tabular}{l|r} 
psi & 2.90 \\
\hline in/lbs & $4^{\prime \prime} / 145$ \\
\hline
\end{tabular}

\section{Exhaust gas heat exchanger}

\begin{tabular}{l|c|c}
\hline Type & & shell-and-tube \\
\hline PRIMARY: & & \\
\hline Exhaust gas pressure drop approx & psi & 0.22 \\
\hline Exhaust gas connection & in/bs & $12^{\prime \prime} / 145$ \\
\hline SECONDARY: & & \\
\hline Design pressure of hot water & psi & 87 \\
\hline Pressure drop hot water circuit & psi & 2.90 \\
\hline Hot water connection & inflbs & $4^{\prime \prime} / 145$ \\
\hline
\end{tabular}


Recoverable thermal output $=\quad 5.695 \mathrm{hBTJ} / \mathrm{hr}$

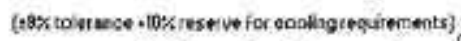

Hot water flow rate $=316.4 \mathrm{GPM}$

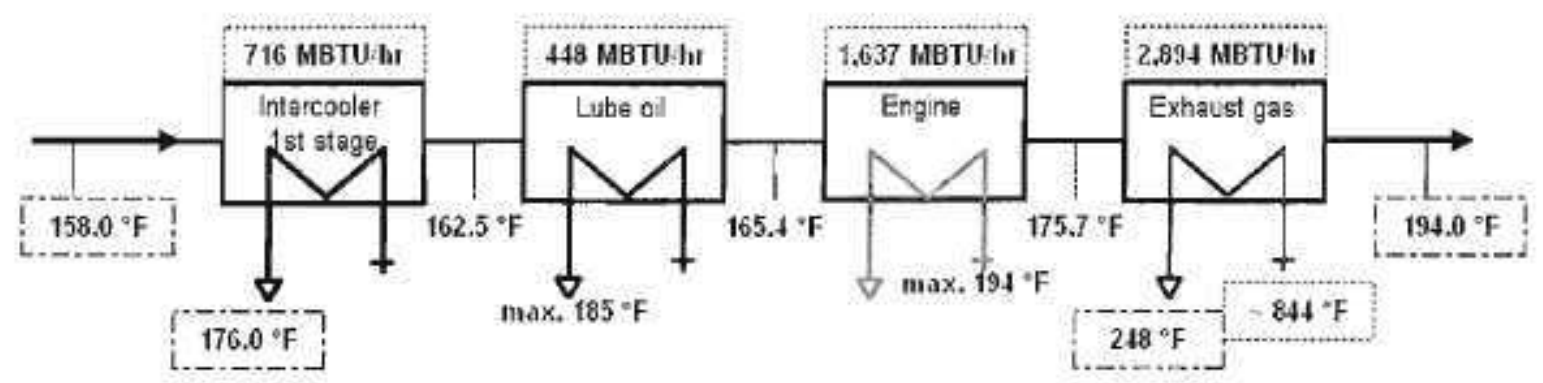

\section{Low Temperature circuit (calculated with Glykol 37\%)}

Heat to be dissipated $=345$ MBTU ht

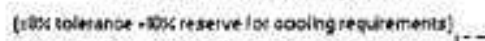

Cooling water flow rate $=88.1 \mathrm{GPM}$

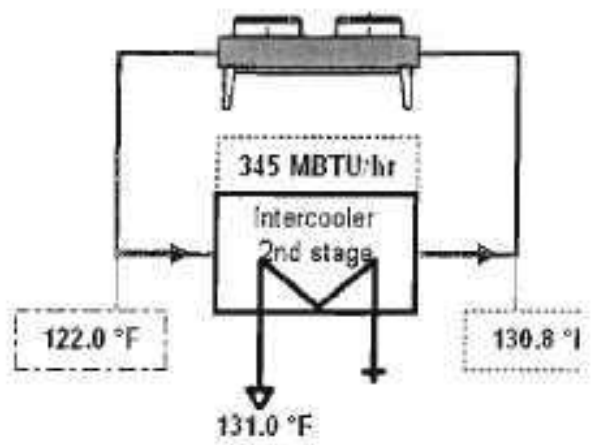




\subsection{Technical parameters}

All data in the technical specification are based on engine full load (unless stated otherwise) at specified temperatures as well as the methane number and subject to technical development and modifications. For isolated operation an output reduction may apply according to the block load diagram. Before being able to provide exact output numbers, a detailed site load profile needs to be provided (motor starting curves, etc.)

All pressure indications are to be measured and read with pressure gauges (psi.g.).

(1) At nominal speed and standard reference conditions ICFN according to DIN-ISO 3046 and DIN 6271 , respectively

(2) According to DIN-ISO 3046 and DIN 6271 , respectively, with a tolerance of $+5 \%$

(3) Average value between oil change intervals according to maintenance schedule, without ofil change amount

(4) At p. f. $=1.0$ according to VDE 0530 REM / IEC 34.1 with relative tolerances

(5) Total output with a tolerance of $+/-8 \%$

(6) According to above parameters (1) through (5)

(7) Only valid for engine and generator; module and peripheral equipment not considered

(8) Exhaust temperature with a tolerance of $+1-5 \%$

\section{Radio interference level}

The ignition system of the gas engines complies the radio interference levels of CISPR 12 and EN 55011 class $\mathrm{B},(30-75 \mathrm{MHz}, 75-400 \mathrm{MHz}, 400-1000 \mathrm{MHz})$ and $(30-230 \mathrm{MHz}, 230-1000 \mathrm{MHz})$, respectively.

\section{Definition of output}

- ISO-ICFN continuous rated power:

Net break power that the engine manufacturer declares an engine is capable of delivering continuously, at stated speed, between the normal maintenance intervais and overhauls as required by the manufacturer. Power determined under the operating conditions of the manufacturer's test bench and adjusted to the standard reference conditions.

- Standard reference conditions:

Barometric pressure:

Air temperature:

Relative humidity:
$14.5 \mathrm{psi}$ ( $1000 \mathrm{mbar})$ or $328 \mathrm{ft}(100 \mathrm{~m})$ above sea level $77^{\circ} \mathrm{F}\left(25^{\circ} \mathrm{C}\right)$ or $298 \mathrm{~K}$

$30 \%$

- Volume values at standard conditions (fuel gas, combustion air, exhaust gas)

Pressure:

Temperature:

1 atmosphere (1013.25 mbar)

$60^{\circ} \mathrm{F}\left(15.56^{\circ} \mathrm{C}\right)$

\section{Output adjustment for turbo charged engines}

Standard rating of the engines is for an installation at an altitude $\leq 1640.5 \mathrm{ft}(500 \mathrm{~m})$ and an air intake temperature $\leq 86^{\circ} \mathrm{F}\left(30^{\circ} \mathrm{C}\right)$.

Derating:

$>1640.5 \mathrm{ft}(500 \mathrm{~m}):$ up to $1.2 \% / 328 \mathrm{ft}(1.2 \% / 100 \mathrm{~m})$

$>86^{\circ} \mathrm{F}\left(30^{\circ} \mathrm{C}\right)$ : up to $0.89 \% /{ }^{\circ} \mathrm{F}\left(1.6 \% /{ }^{\circ} \mathrm{C}\right)$ and over $104^{\circ} \mathrm{F}\left(40^{\circ} \mathrm{C}\right) 1.11 \% /{ }^{\circ} \mathrm{F}\left(2 \% /{ }^{\circ} \mathrm{C}\right)$ 
If the actual methane number is lower than the specified, the knock control responds. First the ignition timing is changed at full rated power. Secondly the rated power is reduced. These functions are done by the engine management.

\section{Parameters for the operation of GE Jenbacher gas engines}

The following "Technical tnstruction of GE JENBACHER" forms an integral part of a contract and must be strictly observed: TI 1100-0110 - TI 1100-0112 


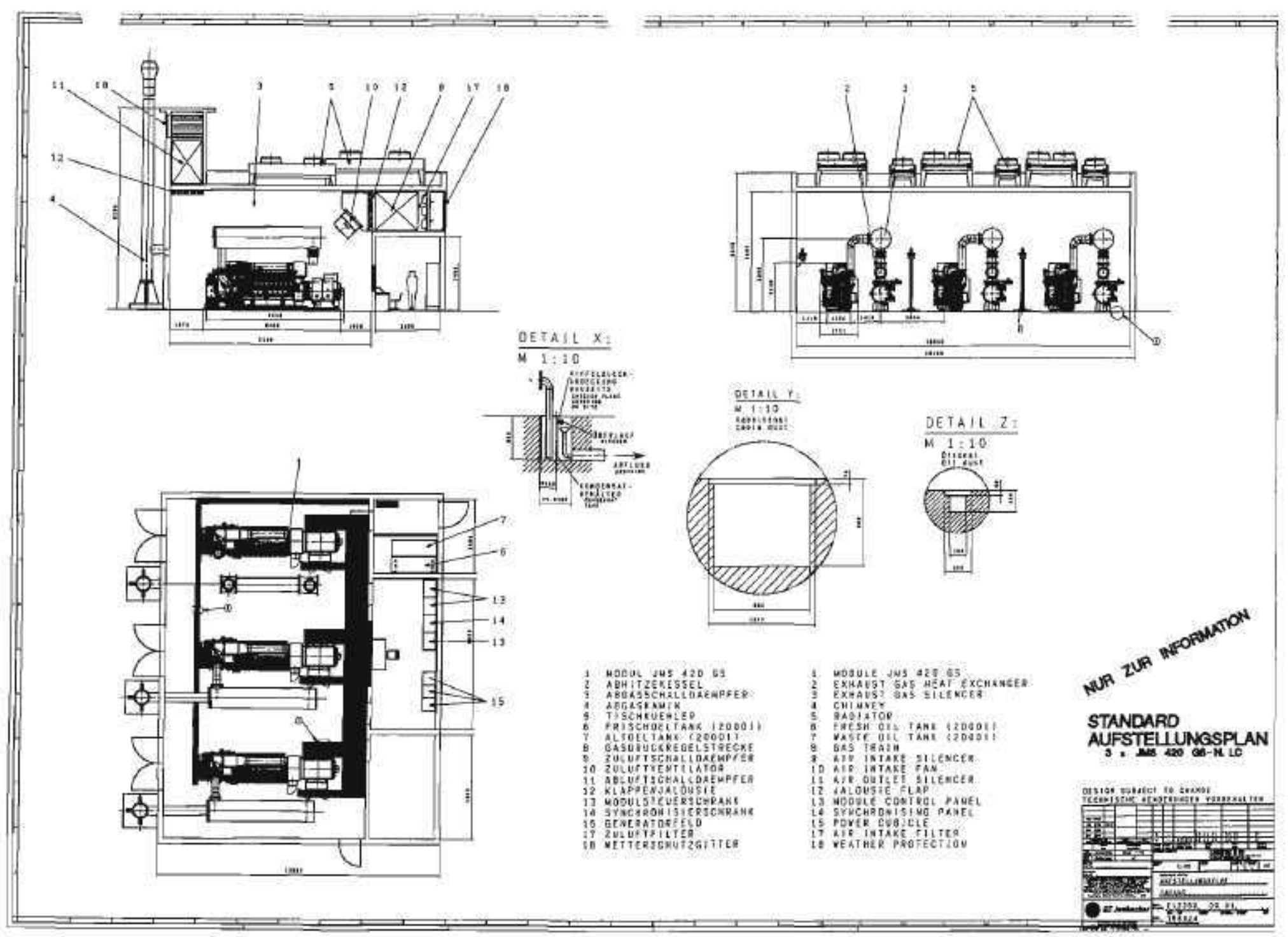




\title{
(es
}

\section{Gas Genset Controls}

\author{
Genset Control Panel (GCP2) \\ with PCS ${ }^{\text {Tu }}$ (PowerCommand Supervisor)
}

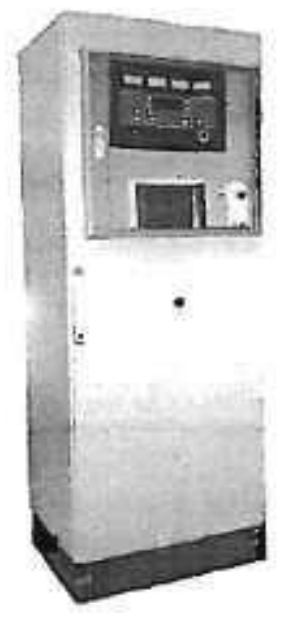

\section{Description}

The Cummins Power Genset Control Panel (GCP2) is an integrated monitoring and control system for the Cummins Lean Bum Natural Gas Gensets. It is a free standing control panel consisting of a Human Machine Interface (HMI), the Cummins PowerCommand Supervisor ( $\mathrm{PCS}^{\mathrm{TM}}$ ), and a Programmable Logic Controller. The GCP2 can be located either with the genset or remotely up to $40 \mathrm{~m}$ (130 ft) from the genset.

The Control is designed and tested to operate the generator set in single unit isolated bus, multiple unit isolated bus fload share) and utility parallel modes.

The PowerCommand control is UL508 Listed. All Cummins Power Generation systems are backed by a comprehensive warranty program and supported by a woridwide network of 170 distributors and service branches to assist with warranty. service, parts, and planned maintenance support.

\section{Features}

Cummins PowerCommand Supervisor (PCS) - The PowerCommand Supervisor Genset Control and protection system includes both analog and digital metering, digital voltage regulation, Power Command Digital Paralleling controls including synchronizing, load sharing, and importlexport controls, Amp Sentry overcurrent protection, and genset fault monitoring.

Color Touchscreen Interface - Displays both engine and generator information. The touchscreen is provided to allow operator monitoring and control of the generator set and includes all information for the complete generator set including the altemator, engine, and auxiliary devices.

Programmable Logic Controller (PLC) - Functions as the communications gateway between the engine control, generator control, touchsrcreen, and auxiliary systems.

Network Capability - Standard Modbus Plus communications allow remote monitoring and control of the genset.

Emergency Stop Switch - Two position "mushroom" head switch for immediate shutdown in an ernergency condition. Capability for additional remote Emergency Stop Switches

Temperature Rated - Designed to operate in between 0 $50^{\circ} \mathrm{C}\left(32-122^{\circ} \mathrm{F}\right)$

Genset to GCP Harness - Standard 5 meter $(10,15,20$, 30 , and $\mathbf{4 0}$ meter hamesses optional)

Enclosures - Steel enclosure minimum 14 gatige sheet metal with window for access to operation and monitoring of the generator set. it is environmentally protected to IP54/NEMA 3R

Warranty and Service - Backed by a comprehensive warranty and worldwide distributor service network. 


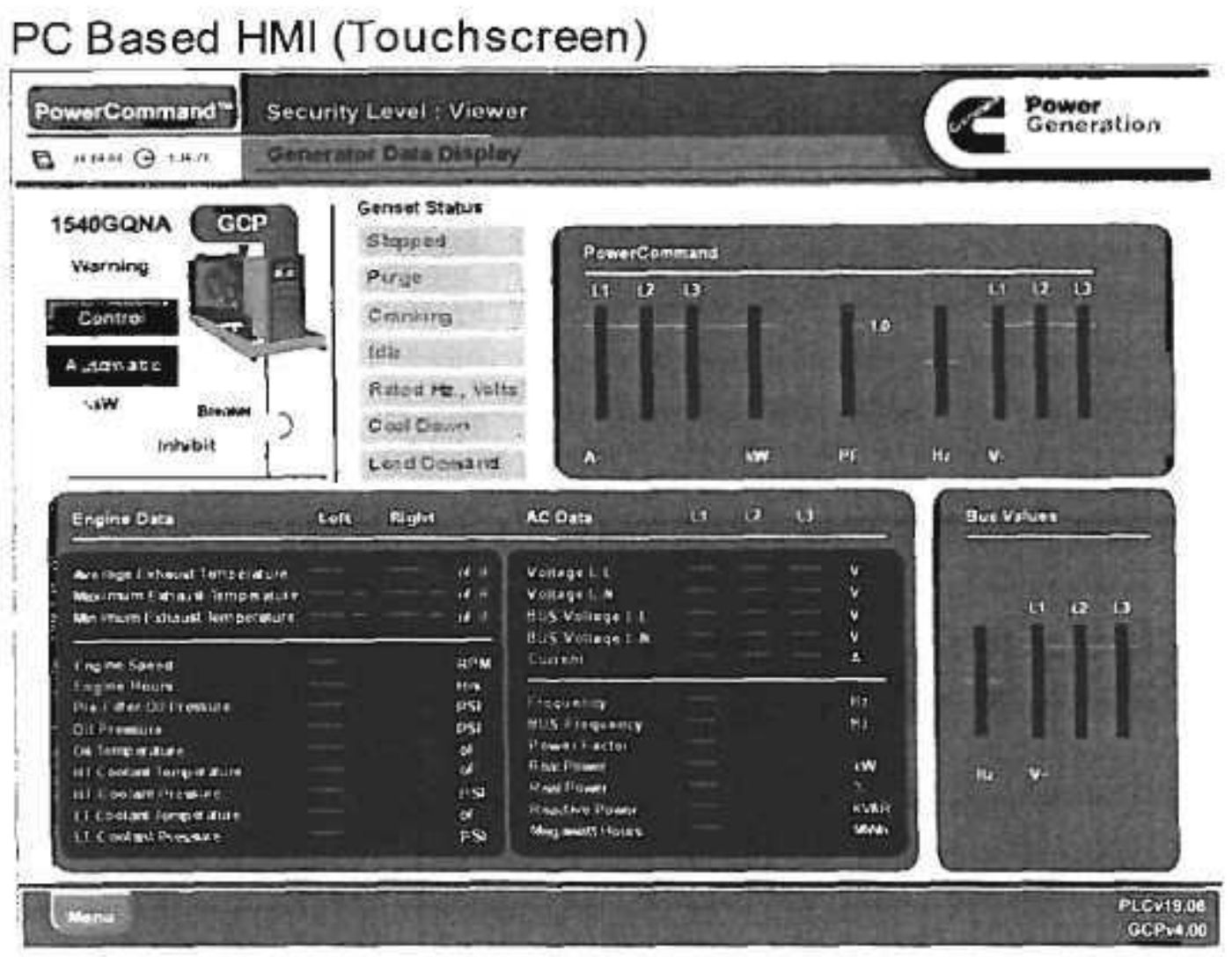

The HMI is a combined industrial PC and 10.4" (264 mm) color touchscreen for monitoring and control of the generator set. The screen is mounted on a console on the front of the GCP2 with gaskets between the touchscreen and the enclosure for environmental protection. It is complete with a real tirne clock, with separate battery backup and incorporates flash memory.

The HMl's main role is to read and display data, the genset is still available and fully protected in the unlikely event of any loss of communication or function. Fault information and real-time data originates from the PCS, engine controller or PLC. The screens HMI will display a mixture of data from some or all of these devices.

There is one genset summary screen (with a popup control screen) and also an alarm summary and history screen. All the principie data required by an operator is available for these three screens.

There is a popup menu providing access to the genset surnmary screen, alarm screens, units selection, security access, operator display languages (English, French, German, Portuguese, Spanish and Dutch) and also restricted access to eight sevice screens.

The main screen is designed to enable the operator to determine the current genset status. It further allows access to the data embedded in the layered screens.

\section{Programmable Logic Controller (PLC)}

The PLC uses a DIN rail mounted card and rack arrangement to allow easy servicing and identification of systern components.

Other features to enhance serviceability include:

- Capability for on-line prograrnddata changes using a personal computer directly connected to the PLC,

- LED status indicators on each board for use in diagnosis of system condition and board level service,

- WO cards include integral surge suppressors for greater system reliability.

2005 Cummins Power Generation

Specifications subject to change without notice

S-1518 (10/05) 


\section{PowerCommand Supervisor}

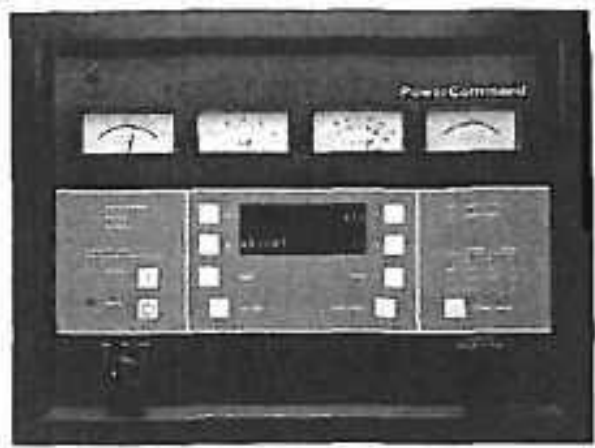

The PCS is a microprocessor based genset monitoring, metering and control system. It is integrated into the GCP2 and incorporates the following key features ;

- Voltage Regulator

- Synchronizer (Freq, Phase \& Voits)

- Iso-Bus kW and kVAR load sharing

- Utility Paralleling kW Load Control

- Utility Paralleting Power Factor Controi

- Altemator Metering

- AmpSentry ${ }^{T M}$ Altemator Protection

- Overłoad,

- Overcument [51].

- Short cirasit [50].

- High AC Volts [59],

- Low AC Volts [27].

- Under frequency [81u].

- Sync Cneck, Fail to Sync,

- CB Fail to Close,

- Reverse Power [32].

- Loss of Excitation [40],

- Phase Rotation

\section{Environmentally Hardened Enclosure}

The front panel of the unit is formed by a single membrane that covers the entire surface. The front face is easy to clean and impervious to water spray, dust and oilexhaust residue. Switches for control are incorporated into the door which is gasketted with a dual moisture and RFVEMI gasket to protect intemal components from air bom contaminants.

\section{Control Switches and Functions}

\section{RUN/OFFIAUTO Mode Control Switch}

An oil tight three position switch starts and stops the generator set locally or enables stait/stop control from a remote position.

It provides the following functions:

- The 'OFF' position de-energizes all primary DC circuits. When the switch is in this position, the non-automatic indicator will flash continuously.

- The 'RUN' position energizes the control and initiates the genset starting operation.

- The 'AUTO' position enables the control to receive a start signal from a remote location.

Note: The non-autornatic indicator will flash when the switch is in the "RUN" or "OFF" position. 


\section{System Control}

Control arrows on the screen lead the operator to information. The control switches provide the operator with a positive indication that the switch is operated. The switches are totally sealed and designed to provide reliable service.

\section{Menu selection Switches}

These four switches allow the operator to select menu-driven control and monitoring information.

\section{Menu 'HOME' Switch}

Retums to the main menu selections screen regardless of the position in the menu logic.

Panel Lights Switch

Tums the back-lit panel ilimination on and off for easy reading of the entire fascia in dark conditions. This feature automatically switches off after 5 minutes.

\section{Test Switch}

Prompts the PowerCommand Control to perform a self test and displays all fault messages.

\section{Reset Switch}

Clears the digital display and status panel and allows the genset to start after a fault condition has been corrected.

\section{Adjustment Menu}

Allows the operator to set basic genset parameters. Adjustments are limited to help prevent operator error and potential darnage to equipment. Critical parameters are adjustable only via a secunty access code.

Adjustrnerits include.

- Voltege $(+/ .5 \%)$

- Automatic Voltage Regulator Gain (access code protected)

Critical service level adjustments are possible oniy after entering an access code. All adjustments are made through digital raisehower switches from the front of the facia.

The adjustment being digitally displayed

\section{External Control Adjustments}

Adjustments for automatic voltage regulation are perfomed directly at the control facia by using the security code without the need to enter the GCP2 enclosure.

\section{Alarm and Status Message Display}

To compliment the HMI screen displays, PCS check data displays are provided for all critical genset parameters.

Digital messages provide a ciear indication of potental problems. A two line 16 character-per-tine, LED alphanumeric screen displays alarm and status messages along with data regarding $\mathrm{AC}$ output.

\section{Status Indicators}

Three dual element LED indicating lamps provide basic genset status data on the facia. Solid state indicators on internal circuit boards provide further status and diagnostic data.

Non-Automatic Indicator

When the Run/Ort/Auto switch is in the OFF or RUN position the red non-automatic indicator will flash on and off.

Warning Indicator

The amber fight indicates the status screen is displaying a warning condition. The reset switch is used to clear the message after the waming condition is corrected. 


\section{Shutdown indicator}

A red light indicates the status screen is displaying a shutdown condtion. The reset switch is used to clear the message after the shutdown condition is corrected.

\section{Generator Set Monitoring - Warning and Shutdown Messages}

The digital display provides status of the following critical engine functions

- Battery voltage

- Speed

- Overspeed

- Magnetic pick up failure (shutdown)

On sensing a waming or shutdown condition the control displays the waming or shutdown message, lights the waming or shutdown indicator lamp on the front of the facia and displays a code number which is interpreted by the PLC and displayed at the HMI in an easy to understand fomat. These codes are also displayed on the PCC facia and can be cross-referenced using the genset manual.

The control has provisions for four programmable faut conditions. These may be either warning or shutdowa conditions. Labels for customer faults can be programmed into the control.

The control maintains an historical data log of the latest alam and status conditions on the genset

\section{Historical data}

The control displays the last 20 alamn and/or shutdown messages

\section{AC Output Metering}

Combines digital and analog metering to provide accurate digital readouts pius analog indication of trends and operating conditions.

\section{Analog Meters}

Analog metering on the control facia provides clear indication of generator set stability from a 'walk by' perspectiva to avoid the requirement to start the HMl screen if it is in screen save mode.

The kilowatt meter and ammeter are scaled in percent of AC output for easy recognition of gensel status and load level $0.90 \%$ of rating ; green, $90-100 \%$ of rating ; amber $=100 \%$ of rating ; red.)

Kilowatt Meter

Indicates 3-phase AC power output as a percent of rated load. Provides a true indication of total kW load on the genset regardless of the load power factor.

Scale $0-125 \%$ of rated

Accuracy is $+1-5 \%$

Frequency Meter

Indicates genset output frequency in hertz. Calculated frequency is based on engine speed and aftemator voltage zero-crossing and is not affected by voltage waveform distortion caused by non-linear loads.

Scale is $45.65 \mathrm{~Hz}$.

Accuracy is $+1-5 \%$.

AC Voltmeter

Dual scale $A C$ voltmeter indicates alternator output voltage.

Accuracy is $+1-2 \%$

Scales are 0-300VAC, 0-6COVAC, 0 - 400 VAC, 0 - 750VAC, $0-5260 \mathrm{VAC}$, $0-15,000$ VAC. 
AC Ammeter

Indicates current output in percent of maximum rated standby current.

Accuracy is $+1-2 \%$

Scale is $0-125 \%$

Phase Select Switch

Allows the operator to select the phase monitored by the analogue ammeter and voltmeter. LED indicators display which phase is being monitored and which voltage scale is applied.

\section{Digital Metering}

The digital metering display provides access to afternator performance data and a more accurate readout of the AC output information displayed on the analogue meters. The followirg outputs are displayed:

- Genset Output Voltage (3-phase, line to line or line to neutral)

- Genset output current (3-phase)

- Power factor (0 to 1, leading or lagging)

- AC kilowatts

- AC kilowatt-hours

- Allemator exciter duty and govemor duty (\%)

- Genset output frequency $(\mathrm{Hz})$

The voltage and current data for all three phases is displayed simultaneously on a single screen so lead and voltage balance is readily apparent

\section{Digital Voltage Regulation, Synchronizing and Load Sharing Controis}

The PCS module includes all voltage regulation, synchronizing and load sharing control required for isolated or infinite bus paralleling applications, including demanding UPS and non-linear load applications.

\section{Applications and Performance}

Paralleling

Isolated Bus Paralleling control (set to set synchronising, isochronous kW and kVAR load sharing) and Base Load Utility Paralleing (synchronising to utility, base load $\mathrm{kW}$ control, VAR/PF controi) features are provided.

Isochronous Real Load Sharing

Load sharing to within as low as $1 \%$ of equal. Load sharing controls operate directly on the engine governor actuator to provide zero droop in frequency for laads rom zero to $100 \%$ of rated genset capacity.

Droop Real Load Sharing

Control may be configured for operation in droop mode, adjustable for no load to full load droop from $1 \%$ to $10 \%$

Isochronous Reactive Load Sharing

Load sharing to within as low as $1 \%$ of equal. Load shanng controls operate directly on the excitation system to provide zero droop in voltage for loads to $100 \%$ of rated genset capacity.

Droop Reactive Load Sharing

Control may be configured for operation in droop mode, adjustable for no load to full load droop from $1 \%$ to $10 \%$.

\section{Synchronizer}

Range:

The synchroniser can drive the genset frequency and voltage to a bus value which is $-10 \%$ to $+10 \%$ of selected voltage and frequency. The ramp speed for matching is $4 \%$ per second.

Frequency differential :

the set is controlled to match the bus frequency.

Voltage differential:

the set voltage is controlled to within $1 \%$ of system bus voitage with checks for correct phase rotation to bus. 
Permissive protection:

Adjustable for a phase difference of 5 to 20 degrees with phase difference decreasing. Time delay is adjustable from 0.5 to 5 seconds

Control System :

Automatically rosets bus frequency and voltage to preset values after the paralieling breaker cioses.

'Dead Bus' Sensor :

Allows ciosure of the generator set to an inactive system bus.

\section{Battery Monitoring System}

The control continualfy monitors the battery charging systern for low and high DC

voltage and runs a battery load test every time the engine is started. Functions and messages include:

- Low DC voltage (battery voltage less than $25 V D C$ except during engine cranking)

- High DC voltage (battery voltage greater than 32 VDC)

- Weak battery (battery voltage less than 14.4 VDC for more than 2 seconds during engine cranking).

\section{Warning and Shutdown Messages}

\section{Overload}

When total KW load exceeds $100 \%$ of the standby rating of the genset (110\% of prime rating) for 5 seconds a load shed signal is issued and a waming alam activated

Overcurrent (51)

When the current on any phase exceeds $110 \%$ of the genset rated current for more than 60 seconds a waming alam is activated.

Overcursent (51)

The genset is shutdown when the current on any phase is between $110-175 \%$ of rated and the time/current integral approaches altemator themal limits.

Short Circuit (50)

The genset is shutdown when the current on any phase exceeds $175 \%$ of rated and the time/current integral approaches alternator thermal limits.

High AC Voltage (59)

Genset is shutdown when AC voltage exceeds $110 \%$ for 10 seconds or with no delay when voltage exceeds $130 \%$ of nominal.

Low AC Voltage (27)

Genset is shutdown when AC voltage falis below $85 \%$ of rated voltage for more than 10 seconds.

UnderFrequency $(81 \mathrm{U})$

Genset is shutdown when AC frequency fals below $90 \%$ of tated frequency for more than 20 seconds.

Reverse Power (32)

Genset is shut down when kW flow into the genset exceeds an adjustable set point ( 5 - $15 \%$ of genset rating) for an adjustable amount of time $(1-15$ seconds).

Loss of Excitation (40)

Genset is shut down when KVAR is less than 0.16-0.41 per unit KVAR (adjustable) for more than 2 - 10 seconds (adjustable). 


\section{AmpSentry Protection}

AmpSentry protection is a comprehensive power monitoring and control system integral to the PCC that guards the electrical integrity of the alternator and power system from the effects of overcurrent, short circuit, overhunder voltage, underfrequency and overload. Current is regulated to $300 \%$ for both singie phase and 3 phase faults when a short circuit condition is sensed. An overcurrent alarm will sound if the genset is operating for an extended period at a potentially damaging current level, to warn the operator of an impending problem before it causes a system failure. If an overcurnent condition persists for the time pre-programmed in the time cument characteristic for the alternator, the PMG excitation system is de-energised to avoid alternator darnage.

The overcurrent protection is time delayed in accondance with the alternator thermal capacity allowing current to flow until secondary fuses or circuit breakers operate, isolating the fault and thus achieving selective co-ordination (discrimination). This enhances power service continuity by eliminating the need for a main line breaker mounted on the genset for genset protection and the possibility of nuisance tripping of that breaker.

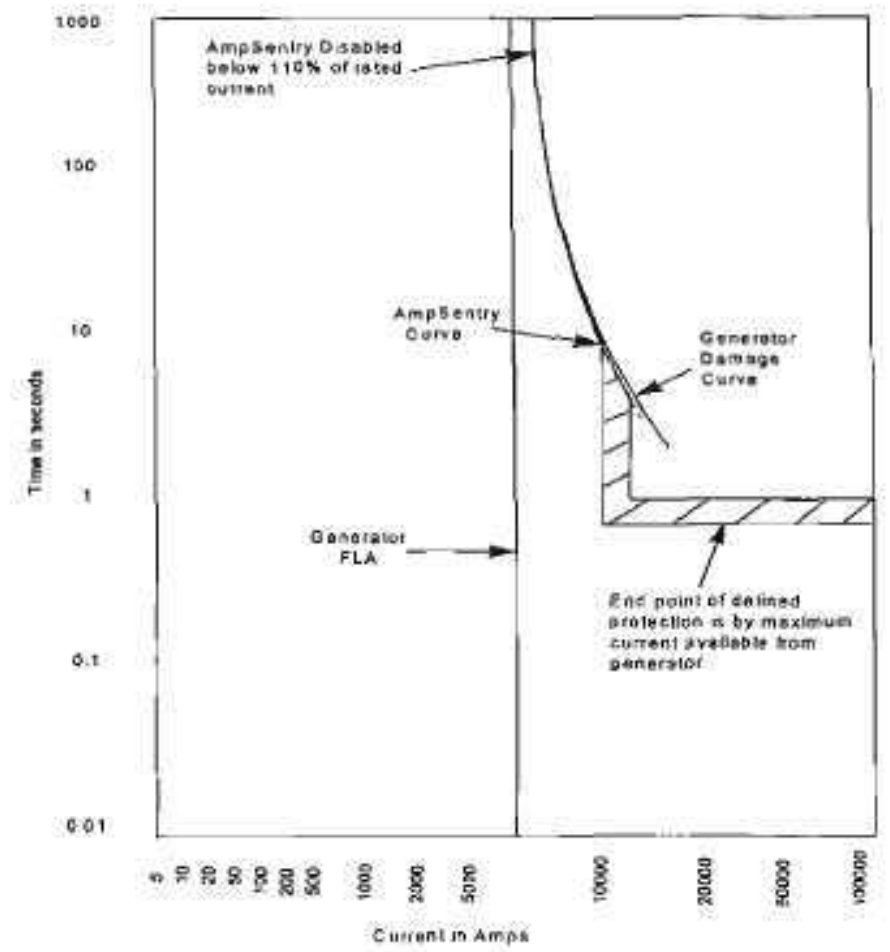

AmpSentry provides excellent, matched protection for the altemator without the danger of nuisance tripping. The exact time current characteristics of AmpSentry are shown in the PowerCommand Control AmpSentry Time-Over-Current Characteristic Curve, Form Number R-1053.

After the fault is cleared AmpSentry Protection softly loads the genset by a controlled ramping of output voltage to rated level, allowing the genset to resume normal operation without polentially damaging voltage overshoot:

Fixed overfunder voltage and under frequency time delayed set points also provide a degree of protection for lead equipment, Over/under voltage conditions trigger a shutdown message on the digital display screen. Under frequency condition prompts both a waming and shutdown message depending upon the length of time and magnitude of variance under rated frequency.

AmpSentry Protection includes an overioad signal that can be used in conjunction with proprietary transfer switches to automaticaily shed load, preventing a potential genset shutdown. The overload signal is programmable for operation at a specifio $\mathrm{kW}$ level, on the basis of an under frequency condition, or both. 

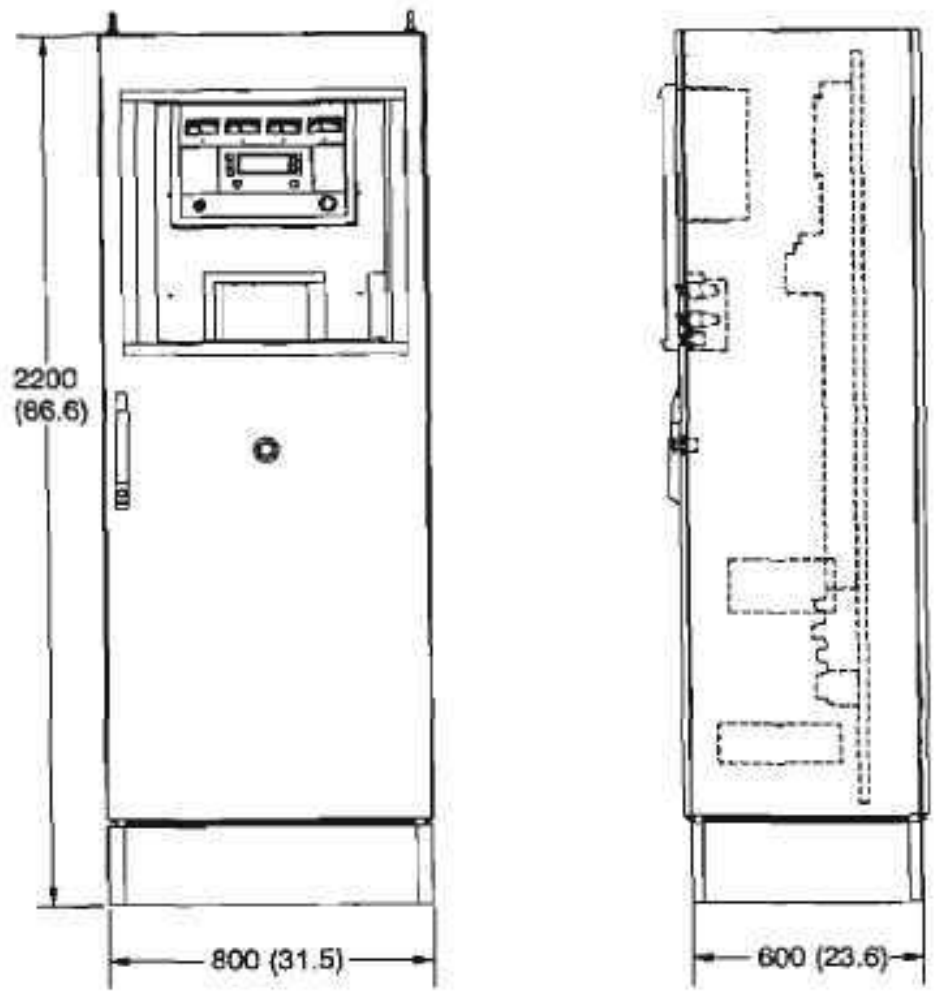

See your distributor for more information.

\section{Power}

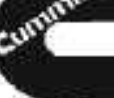

Generation

Cummins Power Generation

$140073^{\text {"11 }}$ Avansie N.E

Minneapolis, MN 55432 USA

Telephone: +1 (763) $574-5000$

Fax +1 (763) 574-5298

Emait: ask.powergengeummins,com

Wab: www cumminspower com

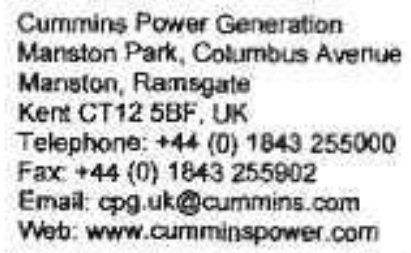

Manston Park, Columbus Avertue

Cummins Power Generation

8 Tanjong Penjurs

Singapore 6099019

Telephone: +65 6285-0155

Telefax + $056266-0432$

Email: mktoggsing cummins.com

Web: www cumminspower.com

Cummins and PowerCommand are registered trademarks of Cummins Inc. AmpSentry is a trademark of Cummins inc.

Imporlant: Back feed to a utility system can cause electrocution and/or property damage. Do not connect to any building's electrical systern excopt through an approved device or after building main switch is open, 


\section{Generator set specifications}

Governor regulation class

Voltage regulution, no load to fult losd

Random voltage variation

Frequency reguiation

Random frequency variation

Fiadio frequency ernissions compliance

Single step load pickup
ISO 8528 Part 1, Class 61 with exceptions - see PTS (Prototype Test Support Data Sheet

$\pm 0.5 \%$

$\pm 0.5 \%$

lsochronous

$\pm 0.25 \%$

IEC 801.2 through IEC 801.5; MEL STD 461C, Part 9

See PTS data sheet for details

\section{Engine specifications}

\begin{tabular}{l|l} 
Design & 4 cycle, v-block, turbocharged low tecnperature aftercooled \\
\hline Bore & $180 \mathrm{~mm}(7.09$ in) \\
\hline Stroke & $200 \mathrm{~mm}(7.87$ in) \\
\hline Displacement & 91.6 liters ( 5590 in3) \\
\hline Oyjinder block & Cast iron, V18 \\
\hline Battery charging alternator & None \\
\hline Starting voitage & 24 volt negative ground \\
\hline Fuel system & Lean bum \\
\hline Igrition system & Individual coil on plug \\
\hline Air cleaner typo & Dry replacsable element \\
\hline Lube oll filter type(s) & Full flow and bypeass fiters \\
\hline Breather. & Breather fitter \\
\hline
\end{tabular}

\section{Alternator specifications}

\begin{tabular}{|c|c|}
\hline Design & Brushiless, 4 pole, revolving fleld \\
\hline Stator & $2 / 3$ pitch \\
\hline Rotor & Two bearing \\
\hline Insulation system & Class F or $\mathrm{H}$ see ADS (Afternator Data Sheet) for details \\
\hline Standard temperature rise & $105^{\circ} \mathrm{C}\left(221^{\circ} \mathrm{F}\right)$ Continuous $840^{\circ} \mathrm{C}\left(104^{\circ} \mathrm{F}\right)$ ambient. \\
\hline Excitar type & PMG (Permanent Magnet Generator) \\
\hline Phase rotation & $A(M), B(M, C M)$ \\
\hline Alternator cooling & Direct drive centrifugal blower fan. \\
\hline AC waveform total harmonic distortion & $<5 \%$ no load to full linear foad, $<3 \%$ for any single harmonic \\
\hline Tetephone influence factor (TIF) & $<50$ par NEMA MG1-22.43 \\
\hline Telephone harmonic factor (THF) & $<3$ \\
\hline
\end{tabular}

\section{Available voltages}

$60 \mathrm{~Hz}$ Three phase fine-neutral/line-line

$.240 / 416 \quad .254 / 440 \quad .277 / 480$

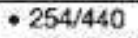

- $7620 / 73200$

- 347/600
- $7970 / 13800$

50 Hz Three phase line-noutral/ine-line

$\begin{array}{llll}\cdot 220 / 380 & \cdot 230 / 400 & \cdot 240 / 415 & \cdot 254 / 440\end{array}$

- $1905 / 3300 \quad \cdot 3640 / 6300 \quad \cdot 3810 / 6600$

- $5775 / 10000$

Wote: Some voltages may not be availabie on all models, Consult factory for availability.

\section{Generator set options and accessories}

\section{Englne}

DNO, $250 \mathrm{mg} / \mathrm{Nm}$ ' $0.5 \mathrm{~g} / \mathrm{hp}-\mathrm{hr}^{2}$

№, $350 \mathrm{mg} / \mathrm{Nm}^{2}(0.9 \mathrm{~g} / \mathrm{hp}-\mathrm{hr})$

$\square \mathrm{NO}_{2} 500 \mathrm{mg} / \mathrm{Nm}^{\prime}$ (1.2 g $\left.\mathrm{gp}-\mathrm{hr}\right)$

D Natural gas fuel methane index es low as 52 for some models

$\square$ High temperature cooling circuit outlet up to $110^{\circ} \mathrm{C}\left(230^{\circ} \mathrm{F}\right)$
口Air starter

DLow BTU Gas

Alternator

$\square 80^{\circ} \mathrm{C}\left(176^{\circ} \mathrm{F}\right)$ rise alternator

D $105^{\circ} \mathrm{C}\left(221^{\circ} \mathrm{F}\right)$ rise alternator

\section{Comtrol panel}

DParalloling bus PTs (69 V, 120

$\mathrm{V}, 240 \mathrm{~V}, 346 \mathrm{~V}$

Generator set

$\square C E$ Certification

Wote: Some options may not be availabie on all models - consult factary for avaltability.

\section{Our energy working for you. rm}

\section{WwW. Cumminspower. com}

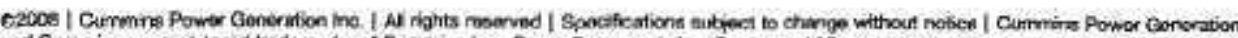

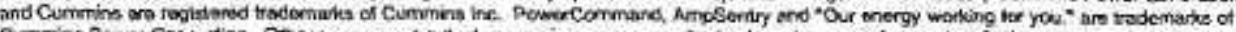

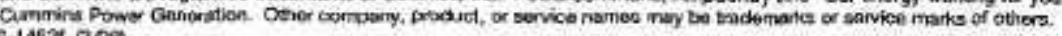

\section{Accessories}

$\square$ Exhaust illencers

$\square$ Ges Train

ㅁRadiators

口Bladder Expansion Tank

$\square$ Heat Exchanger

$\square$ Exhaust Heat Recovery 


\section{Generator control panel}

Stand alone remote mounted cabinet

\section{PC based HMI}

- Micro-processor based graphic interface (touchscreen)

- Layered menus for ease of operation

PLC based auxiliary control

- Communication handling procedures

- Protocol interfaces

- Control of plant auxilianies

Stand alone or parallel operation

- Singie or multi-set isolated bus operations

- Single set base load utility paraleling

- Base load utility paraileling control

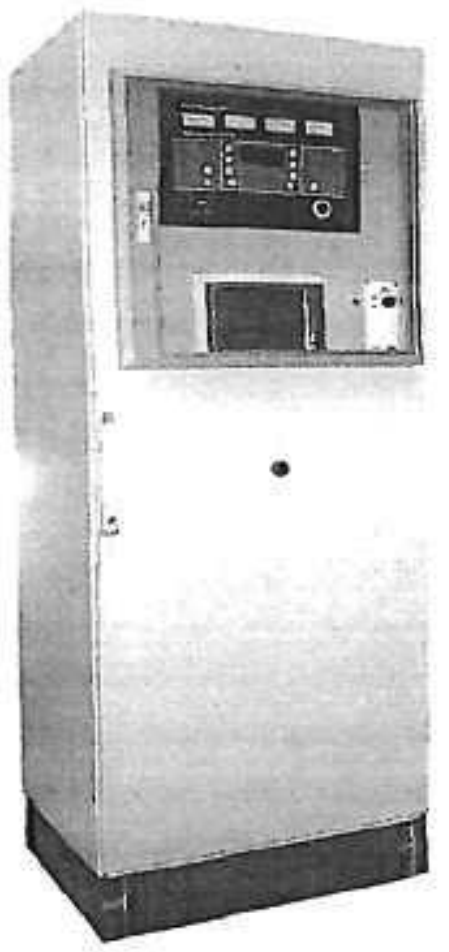

\section{PowerCommand Supervisor}

(PCS)

\section{Features}

- Integrated automatic voltage reguiator

- Speed/hoad bias to engine governor control

- Ampsentry protection guards the electrical integrity of the altemator and power system from the effects of over current, over/unider voltage, under frequency and overload conditions.

- Oll pressure sender fault

- Over current

- Overioad load shed contacts

- Temperature sender fault

- Up to four customer fautt inputs

- Weak battery

Shutdowns

- Emergency stop

- Fail to crank

- High AC voltage

- High coolant temperature

- Control components are designed : Low AC voitage typical in generator sets

Standard control description

- Analog \% of current meter (amps)

- Analog \% of load meter (kW)

- Analog AC frequency meter

- Analog AC voltage meter

- Cycle cranking control

- Digital cisplay parel

- Emergency stop switch

- Idile mode control

- Menu switch

- Panel backlighting

- Remote starting, 12 V, 2 wire

- Reset switch

- Run-olf-auto switch

- Sealed tront panel, gasketed door

- Self diagnostics

- Separate customer interconnection box

- Voltmeter/ammeter phase selection

- Low coolant level (option for alarm only)

- Low oil pressure

- Magnetic pickup failure

- Overcrank

- Over current

- Overspeed

- Short circuit

- Underfrequency

Standard performance data AC alternator

- Current by phase

- Kilowatts

- Kilowatt hours

- Power factor

- Voltage line to line

- Voltage line to neutral

Engine data

- Battery voltage

- Coolant temperature

- Engine running hours

Standard protection functions - Engine starts counter

Warnings

- High coolant ternperature

- High DC voltage

- Oil pressure

- Oil temperature

- RPM

- Low coolant temperature

- Low DC voltage

- Low fuel-day tank

- Low oll pressure

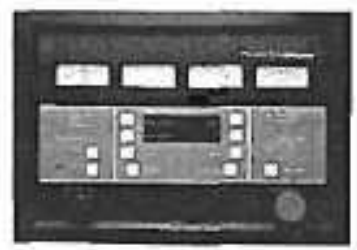




\section{Base load (continuous) rating definition}

Applicable for supplying power continuously to a constant load up to the full output rating for unlimited hours. No sustained overload capability is available for this rating. Consult authorized distributor for rating. (Equivalent to Continuous Power in accordance with ISO 8528 , iSO 3046 , AS2789, DiN6271, and BS5514).

\section{Generator set data sheets}

\begin{tabular}{|c|c|c|c|c|c|}
\hline & & & & & \\
\hline Model & $\begin{array}{l}\text { Data } \\
\text { sheet }\end{array}$ & $\mathrm{CR}^{*}$ & $\begin{array}{l}\text { Emisaions } \\
\text { g/hp-hr }\end{array}$ & $\begin{array}{l}\text { LT } \\
\text { ('C) }\end{array}$ & $\begin{array}{l}\mathrm{HT} \\
\mathrm{PO} C)\end{array}$ \\
\hline $1750 \mathrm{GQPB}$ & D-3364 & $11.4: 1$ & 500 & 50 & 103 \\
\hline $1750 \mathrm{GQPB}$ & $D-3365$ & $12: 1$ & 500 & 50 & 103 \\
\hline
\end{tabular}

\begin{tabular}{|c|c|c|c|c|c|}
\hline Model & $\begin{array}{l}\text { Data } \\
\text { sheet }\end{array}$ & CA* & $\begin{array}{l}\text { Emlssions } \\
\mathrm{mg} / \mathrm{WM} \text { " }\end{array}$ & $\begin{array}{l}17 \\
\text { (NC) }\end{array}$ & $\begin{array}{l}\mathrm{HT} \\
\text { ( } \mathrm{C})\end{array}$ \\
\hline 1750 GONB & $D-3362$ & $11.4: 1$ & 500 & 50 & 103 \\
\hline 1750 GONB & $D-3363$ & $12: 1$ & 500 & 50 & 103 \\
\hline
\end{tabular}

\section{$60 \mathrm{~Hz}$ pipeline gas}

\begin{tabular}{|c|c|c|c|c|c|}
\hline Modol & $\begin{array}{l}\text { Data } \\
\text { shoet }\end{array}$ & MN* & $\begin{array}{l}\text { Emissions } \\
\text { g/hp-hr }\end{array}$ & $\begin{array}{l}\mathrm{LT} \\
\mathrm{r} C \mathrm{C})\end{array}$ & $\begin{array}{l}\text { NT } \\
\text { (PC) }\end{array}$ \\
\hline 1250 GONA & D-3282 & 60 & 500 & 50 & 95 \\
\hline 1250 GONA & D-3283 & 56 & 350 & 50 & 95 \\
\hline 1250 GQNA & $D-3284$ & 63 & 500 & 50 & 110 \\
\hline 1250 GONA & $D-3285$ & 59 & 350 & 50 & 110 \\
\hline 1250 GQNA & D-3286 & 70 & 500 & 50 & 95 \\
\hline 1250 GONA & $D-3287$ & 66 & 350 & 50 & 95 \\
\hline 1250 GONA & D-3288 & 73 & 500 & 50 & 110 \\
\hline 1250 GQNA & D-3289 & 69 & 350 & 50 & 110 \\
\hline $1750 \mathrm{GQPB}$ & $D-3307$ & 87 & 500 & 50 & 95 \\
\hline $1750 \mathrm{GCPE}$ & D-3308 & 63 & 350 & 50 & 95 \\
\hline $1750 \mathrm{GOPB}$ & D-3311 & 77 & 500 & 50 & 95 \\
\hline $1750 \mathrm{GCPB}$ & $D-3312$ & 73 & 350 & 50 & 95 \\
\hline $1750 \mathrm{GQPB}$ & D- 3313 & 80 & 500 & 50 & 110 \\
\hline 1750 GOPB & D-3314 & 76 & 350 & 50 & 110 \\
\hline 2000 GCPC & $D-3325$ & 78 & 1.2 & 45 & 92 \\
\hline 2000 GQPC & D-3339 & 76 & 0.5 & 50 & 92 \\
\hline
\end{tabular}

- $\mathrm{CR}=$ Compression ratio " $\mathrm{MN}=$ Methane
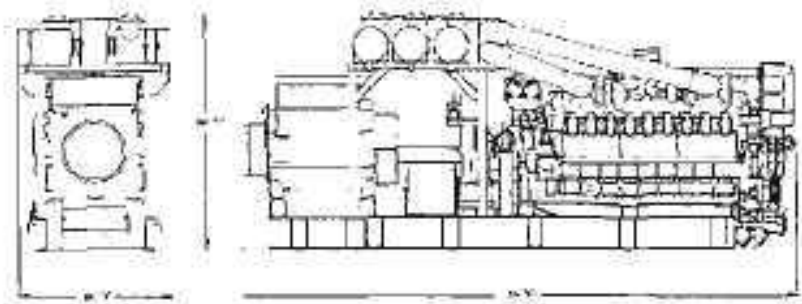

This cutline drawing is to provide representative contiguration details for Modet series only.

See respective moded data sheet for specific moded outline drawing numbec

\section{Do not sese for installation desirn}

\begin{tabular}{|c|c|c|c|c|c|}
\hline Model & $\begin{array}{l}\text { Data } \\
\text { sheet }\end{array}$ & $\mathbf{M W}^{* *}$ & $\begin{array}{l}\text { Emisglons } \\
\text { mg/WM: }\end{array}$ & $\begin{array}{l}\mathrm{LT} \\
\text { ("C) }\end{array}$ & $\begin{array}{l}\text { HT } \\
\text { ('C) }\end{array}$ \\
\hline 1540 GQNA & D-3290 & 52 & 500 & 50 & 95 \\
\hline 1540 GQNA & $D-3291$ & 60 & 500 & 50 & 95 \\
\hline 1540 GONA & $D-3292$ & 56 & 350 & 50 & 95 \\
\hline 1540 GQNA & D.3293 & 6.3 & 500 & 50 & 110 \\
\hline 1540 GQNA & $D-3294$ & 59 & 350 & 50 & 110 \\
\hline 1540 GONA & D.3295 & 70 & 300 & 50 & 95 \\
\hline 1540 GONA & $D-3296$ & 66 & 350 & 50 & 95 \\
\hline 1540 GQNA & D-3297 & 73 & 500 & 50 & 110 \\
\hline 1540 GONA & D-3298 & 69 & 350 & 50 & 110 \\
\hline 1750 GQNB & D. 32999 & 67 & 500 & 50 & 95 \\
\hline 1750 GONB & $D-3300$ & 63 & 350 & 50 & 95 \\
\hline 1750 GQNB & D-3303 & 77 & 500 & 50 & 95 \\
\hline 1750 GCNB & $D-3304$ & 73 & 350 & 50 & 95 \\
\hline 1750 QQNB & $D-3305$ & 80 & 500 & 50 & 110 \\
\hline 1750 GQNB & D-3306 & 76 & 300 & 50 & 110 \\
\hline 2000 GQNC & D-3322 & 73 & 500 & 40 & 92 \\
\hline 2000 GQNC & D-3323 & 70 & 350 & 40 & 92 \\
\hline 2000 GONC & D-3338 & 75 & 250 & 50 & 92 \\
\hline 2000 GQNC & D-3359 & 80 & 500 & 50 & 92 \\
\hline
\end{tabular}

\section{Dimensions and weights}

\begin{tabular}{|c|c|c|c|c|}
\hline Model & 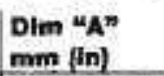 & $\begin{array}{l}\text { Dim "Bn } \\
\text { mm [in] }\end{array}$ & $\begin{array}{l}\text { Dim } \text { ACn }^{n} \\
\text { mm (in) }\end{array}$ & $\begin{array}{l}\text { Wolght" wet } \\
\text { kg flbs) }\end{array}$ \\
\hline $1250 \mathrm{GQNA}$ & $5971(235.1)$ & $1720(67.7)$ & $3136(123.5)$ & $17595(38709)$ \\
\hline $1750 \mathrm{GCPB}$ & $7302(287.5)$ & $1720(67.7)$ & $3136(123.5)$ & $22100(48620)$ \\
\hline 1540 GONA & $5603(220.6)$ & $1720(87,7)$ & $3136(1235)$ & $17057(38516)$ \\
\hline 1750 GQNB & $5921(233.1)$ & $1720(67.7)$ & $3136(123.5)$ & $19633(43192)$ \\
\hline
\end{tabular}

- Weights represent a set with standard features. See outline drawings for weights of other configurations.

\section{Amoricas}

$140073^{r}$ Avenue N.E.

Minneapolis, MN 55432 USA

Phone 7635745000

USA tol-free 8777697669

Fax 7635745298
Europe, CAs, Middle East and Africa

Mariston Park Columbus Ave.

Marston Ramsgate

Kent CT $125 \mathrm{BF}$ United Kingdom

Phone 441843255000

Fax 441843255902
Asia Pactifo

10 Toh Guan Road $\approx 07-01$

II Internationad Tradepark

Singapore 603838

Phone 65 64172388

Fax 6564172399

Waming: Back teed to a utikity systern can cause electrocution and/or property damage. Do not connect to any bulding's electrical system except through an approved device or after bulicing main switch is open.

\section{Our enorgy working for you. $n$}

www.curnminspower.com

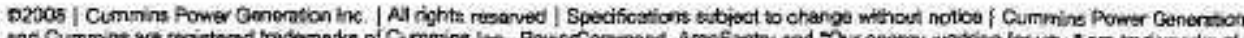

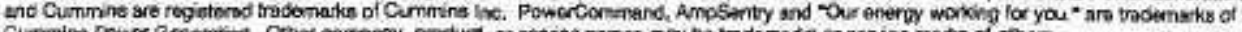

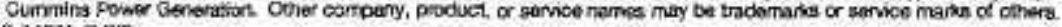
S-1453 (300)

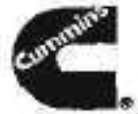

\section{Power Generation}




\section{Model: 2000GQPC \\ Frequency: $60 \mathrm{~Hz}$ \\ Fuel Type: Natural Gas MI 78 * \\ Emissions Performance NOx: $1.0 \mathrm{ghph}$ \\ LT Water Inlet Temperature: $45^{\circ} \mathrm{C}\left(113^{\circ} \mathrm{F}\right)$ \\ HT Water Outlet Temp: $92^{\circ} \mathrm{C}\left(198^{\circ} \mathrm{F}\right)$}

Generator sef data sheet

$2000 \mathrm{~kW}$ continuous

Our chergy working for you.r.
Power Generation
Measured Sound Performance Data Sheet:

Prototype Test Summary Data:

Remote Radiator Cooling Outine:

Fuel Consumption (ISO3046/1)

Fuet Consumption (LHV) $1503046 / 1, \mathrm{~kW}$ (MMBTU/2r)

Mechanical Efficiency ISO3046/1, percent

Electrical Efficiency ISO3046/1, percent

Engine

Engine Manufacturer

Engine Model

Configuration

Displacernent, L (cu.in)

Aspiration

Gross Engine Power Output, kWm (hp)

BMEP, bar (psi)

Bore, mm (in)

Stroke, $\mathrm{mm}$ (in)

Rated Speed, rm

Piston Speed, m/s (ft/min)

Compression Ratio

Lube Oil Capacity, L (qt)

Overspeed Limit, rpm

Regenerative Power. KW

Full Load Lubricating oil consumption, g/kWe-hr (g/hp-hr)

Fuel

Gas supply pressure to engine inlet, bar (psi)

Minimum Methane index

\section{Starting System(s)}

Electric starter voitage, volts

Minimum battery capacity @ 40 deg.C (104 deg.F), AH

Air Starter Pressure, barg (psig)

Air Starter Flow $\mathrm{Nm}^{3} / \mathrm{s}$ (scfm)

\section{Genset Dimensions (see note 1)}

Genset Length, $m$ ( $\mathrm{ft}$ )

Genset Width, $m$ (ft)

Genset Height, $m$ (ii)

Genset Weight (wet). kg (bs)
MSP - 1039

PTS - 269

$0500-5093$

\begin{tabular}{|l|c|l|l|l} 
See Note & $\begin{array}{c}100 \% \text { of Rated } \\
\text { Land }\end{array}$ & $\begin{array}{c}90 \% \text { of Rated } \\
\text { Losd }\end{array}$ & $\begin{array}{c}75 \% \text { of Rnted } \\
\text { Load }\end{array}$ & $\begin{array}{c}\text { sox of Ratad } \\
\text { Load }\end{array}$ \\
\hline $2,4,6,7$ & $5001(17,08)$ & $4555(15.56)$ & $3876(13.24)$ & $2832(9.67)$ \\
\hline $2,4,7$ & $42.2 \%$ & $41.7 \%$ & $40.9 \%$ & $37.8 \%$ \\
\hline $2,4,6,7$ & $40.0 \%$ & $39.5 \%$ & $38.7 \%$ & $35.3 \%$ \\
\hline
\end{tabular}

Cummins

QSV91G

V18

$91.6(5591)$

Turbocharged (1)

$2108(2826)$

$18.5(268)$

$180(7.09)$

$200(7.87)$

1514

$10(1968$

12.5

$550(581)$

1800

N/A

$0.4(0.3)$

$15-.20(2.2-2.9)$

78

24

780

$10.3(150)$

$0,37(780)$

$7.12(23.4)$

$2.16(7.1)$

$2.78(9.1)$

$20705(45,644)$ 


\begin{tabular}{|c|c|c|c|c|c|}
\hline & $\begin{array}{l}\text { See } \\
\text { Notes } \\
\end{array}$ & $\begin{array}{l}100 \% \text { of Rated } \\
\text { Load }\end{array}$ & $\begin{array}{l}90 \% \text { of Rated } \\
\text { Load }\end{array}$ & $\begin{array}{l}75 \% \text { of Rated } \\
\text { Load }\end{array}$ & $\begin{array}{l}50 \% \text { of Rated } \\
\text { Load }\end{array}$ \\
\hline \multicolumn{6}{|l|}{ Energy Data } \\
\hline Continuous Shat Power. kWm (bho) & 2,10 & $2108(2826)$ & $1900(2546)$ & $1586[2126)$ & $1071\{1436\}$ \\
\hline Conbinuous Generator Electrical Output kWe & 6,10 & 2000 & 1800 & 1500 & 1000 \\
\hline Heat Dissipaled in Lube Onl Copler. KW (MMGBTU/h) & 5 & $277(0.95)$ & $287(0.91)$ & $249(0.85)$ & $208(0.71)$ \\
\hline Heat Dissipated in Block, kW (MMBTU/h) & 5 & $503(1.71)$ & $488(166)$ & $445(1.52)$ & $418(1.43)$ \\
\hline Total Heat Rejecled in LT Circult, KW (MMBTUR) & 5 & $219(0.75)$ & $200(0.68)$ & $168(0.57)$ & $127(0.43)$ \\
\hline Total Heat Rejected in HT Circuit, kW (MMBTUm) & 5 & $1096(9.74)$ & $1007(3.44)$ & $855(292)$ & $664(2.26)$ \\
\hline UnbumL, kW (MMBTUM) & 13 & $111(0.38)$ & $104\langle 035\rangle$ & $93(0.32)$ & $66(0.23)$ \\
\hline Heat Radiated to Ambient, KW (MMBTU/h) & 13 & $368(1.26)$ & $337(1.15)$ & $288(0.98)$ & $218(0.74)$ \\
\hline Available Exhaust heat to $105 \mathrm{C}, \mathrm{kW}$ (MMBTU/h) & 5 & $1259(4.30)$ & $1169(3.89)$ & $1016(3.47)$ & $818(2,79)$ \\
\hline \multicolumn{6}{|l|}{ Intake Air Flow } \\
\hline Intake Air Flow Mass, kg/a (b/hr) & 4 & $3.16(24992)$ & $2.84(22514)$ & $2.39(18903)$ & $1.66(13117)$ \\
\hline Intake Air Flow Volume, $\mathrm{m} 3 / \mathrm{s} @ \mathrm{~g}^{\circ} \mathrm{C}$ (scfm) & 4 & $2.44(5449)$ & $2.20(4909)$ & $1.85(4122)$ & $1.28(2860)$ \\
\hline Maximum Air Clearnar Restriction, mmHG (in $\mathrm{H}_{2} \overline{\mathrm{O}}$ ) & & $22.07(11.8)$ & $22.07(11.8)$ & $22.07(11.8)$ & 22 \\
\hline \multicolumn{6}{|l|}{ Exhaust Air Flow } \\
\hline Exhaust Gas Flow Mass, $\mathrm{kg} / \mathrm{s}$ (1b/hr) & 4 & $3.27(25866)$ & $2.94(23311)$ & $2,47(19583)$ & $1.72(13607)$ \\
\hline Exhaust Gas Flow Volume, $\mathrm{m} 3 / \mathrm{s}$ (cfm) & 4 & $6.76(14315)$ & $6.18(13080)$ & $5.30(11225)$ & $3.90(8264)$ \\
\hline Exhaust Temperalure After Turbine, ${ }^{\circ} \mathrm{C}\left({ }^{\circ} \mathrm{F}\right.$ ) & 2 & $458(856)$ & $468(875)$ & $484(903)$ & $529(985)$ \\
\hline Max Exhaust System Back Pressure, mm HG (in $\mathrm{H}_{2} \mathrm{O}$ ) & 6,14 & $37.3(20.0)$ & $37.3(20.0)$ & $37.3(20.0)$ & $37.3(200)$ \\
\hline Min Exhaust System Back Pressure, mmHG (in $\mathrm{H}_{2} \mathrm{O}$ ) & 6,14 & $18.7(10.0)$ & & & \\
\hline \multicolumn{6}{|l|}{ HT Cooling Circuit } \\
\hline HT Circuit Engine Coolant Volume, I (gal) & & $424(112)$ & $424(112)$ & $424(112)$ & $424(112)$ \\
\hline HT Cookant Flow of Max Ext Restriction, $\mathrm{m}^{3} \mathrm{~h}$ (gal/min) & & 70 (309) & $70(308)$ & $70(308)$ & $70(308)$ \\
\hline Maximum HT Engine Coolant In'el Temp. ${ }^{\circ} \mathrm{C}\left({ }^{\circ} \mathrm{F}\right)$ & 8 & $75(187)$ & $75(167)$ & $75(167)$ & $75(167)$ \\
\hline HT Coolant Outlel Temp, ${ }^{\circ} \mathrm{C}$ ( F) & B & $92(198)$ & $92(198)$ & $92(198)$ & $92(198)$ \\
\hline Max Prossure Drop in External HT Clrcuil, bar (psig) & & $1.5(22)$ & $1.5(22)$ & $1.5(22)$ & $1.5(22)$ \\
\hline HT Circuit Maximum Pressure, bar (psig) & & $6.0(87)$ & $6.0(87)$ & $6.0(87)$ & $60\{87\}$ \\
\hline Minimum Static Head, bar (psig) & & $0.5(7)$ & $0.5(7)$ & $0.5(7)$ & $0.5(7)$ \\
\hline \multicolumn{6}{|l|}{ LT Cooling Circuit } \\
\hline LT Circuit Engine Coolant Volume, I (gal) & & $295(78)$ & $295(78)$ & $295(78)$ & $295(78)$ \\
\hline LT Coolant Flow @ Max Ext Restriction, $\mathrm{m}^{3} / \mathrm{h}$ (galmin) & & $50.00(220)$ & $50.00(220)$ & $50.00(220)$ & $5000(220)$ \\
\hline Maximum LT Engine Coolant inlet Temp. ${ }^{\circ} \mathrm{C}\left({ }^{6} \mathrm{~F}\right)$ & 9 & $45(113)$ & $45(113)$ & $45(113)$ & $45(113)$ \\
\hline LT Coolant Outlet Temp, eC ( $\mathrm{F}$ ) Relerence Only & 9 & $47.9(118)$ & $47.7(118)$ & $47.2(117)$ & $46.7(116)$ \\
\hline Max Pressure Drop in External LT Circuit, ber (psig) & & $1.5(22)$ & $1.5(22)$ & $1.5(22)$ & $1.5(22)$ \\
\hline LT Circuit Maximum Pressure, bar (psig) & & $6.0(87)$ & $6.0(87)$ & $6.0\{87\}$ & $6.0(87)$ \\
\hline Minimum Static Head, bar (psic) & & $0.5(7)$ & $0.5(7)$ & $0.5(7)$ & $0.5(7)$ \\
\hline \multicolumn{6}{|l|}{ Emissions } \\
\hline $\mathrm{NO}_{x}$ Emissions wel, ppm & 5 & 168 & 177 & 177 & 178 \\
\hline $\mathrm{NO}_{x}$ Emissions, $\mathrm{mg} / \mathrm{Nm}^{3} @ 5 \% \mathrm{O}_{2}(\mathrm{~g} / \mathrm{hp}-\mathrm{h})$ & 5 & $493(1.00)$ & $511(1.00)$ & $505(1.00)$ & $490(1.00)$ \\
\hline THC Emissions wet. ppm & 13 & 1382 & 1431 & 1524 & 1571 \\
\hline THC Emissions, mg/Nm $@ 5 \% \mathrm{O}_{2}(\mathrm{~g} / \mathrm{hp}-\mathrm{h})$ & 13 & 1473 & 1517 & 1605 & 1607 \\
\hline $\mathrm{CH}_{4}$ Emissions wet, ppm & 13 & 1101 & 1133 & 1202 & 1247 \\
\hline $\mathrm{CH}_{4}$ Emissions, mg/Nm $25 \% \mathrm{O}_{2}(9 / \mathrm{hp}-\mathrm{h})$ & 13 & $1190(2.53)$ & $1217(2.60)$ & $1284(277)$ & $1292(3.00)$ \\
\hline NMHC Emissions wet. ppm & 13 & 279 & 298 & 324 & 324 \\
\hline NMHC Emissions, mg/Nm $25 \% \mathrm{O}_{2}$ (g/hp-h) & 13 & 298 & 315 & 341 & 331 \\
\hline CO Emissions (dry), pprn & 13 & 582 & 575 & 575 & 580 \\
\hline CO Emissions, mg/Nm ${ }^{3}\left(96 \% \mathrm{C}_{2}(g / h p-h)\right.$ & 13 & $975(2.07)$ & $958(2.05)$ & $850(2.05)$ & $927(2.15)$ \\
\hline O2 Emissions (dry). percent & 13 & 9.1 & 90 & 8.9 & 8.5 \\
\hline Particulales PM10, ghp-h & 13 & $<0.06$ & $\mathrm{~N} / \mathrm{a}$ & $n / a$ & $\pi / a$ \\
\hline
\end{tabular}


Genset De-rating

Altitude and Temperature Derate Multiplication Factor

\begin{tabular}{|c|c|c|c|c|c|c|c|c|c|c|c|c|}
\hline \multicolumn{2}{|c|}{ Barometer } & \multicolumn{2}{|c|}{ Altitude } & \multirow{2}{*}{\multicolumn{9}{|c|}{$\begin{array}{l}\text { Table A * } \\
\text { Derate Multiplier with Grid Parallel Operation }\end{array}$}} \\
\hline \multirow{2}{*}{$\frac{\ln \mathrm{Hg}}{207}$} & \multirow{2}{*}{$\frac{\text { mbar }}{701}$} & \multirow{2}{*}{ Feet } & \multirow{2}{*}{$\begin{array}{l}\text { Meters } \\
3000\end{array}$} & & & & & & & & & \\
\hline & & & & 0.75 & 0.75 & & & & & & & \\
\hline 21.4 & 723 & 9022 & 2750 & 0.80 & 0.80 & & & & & & & \\
\hline 22.1 & 747 & 8202 & 2500 & 0.85 & 0.85 & 0.75 & & & & & & \\
\hline 228 & 771 & 7382 & 2250 & 090 & 0.90 & 0.80 & & & & & & \\
\hline 235 & 795 & 6562 & 2000 & 0.95 & 0.95 & 0.85 & 0.75 & & & & & \\
\hline 243 & 820 & 5741 & 1750 & 1.00 & 1.00 & 0.90 & 0.80 & & & & & \\
\hline 250 & 846 & 4921 & 1500 & 1.00 & 1.00 & 0.95 & 0.85 & 0.75 & & & & \\
\hline 25.8 & 872 & 4101 & 1250 & 1,00 & 1.00 & 1.00 & 0.90 & 0.20 & & & & \\
\hline 26.6 & 299 & 3281 & 1000 & 1.00 & 1.00 & 1.00 & 0.95 & 0.85 & 0.75 & & & \\
\hline 27.4 & 926 & 2461 & 750 & 1.00 & 1.00 & 100 & 1.00 & 0.90 & 0.80 & & & \\
\hline 28.3 & 954 & 1640 & 500 & 1.00 & 1.00 & 1.00 & 1.00 & 0.95 & 0.85 & & & \\
\hline 29.1 & 583 & 820 & 250 & 1.00 & 1.00 & 1.00 & 1.00 & 1.00 & 0.90 & & & \\
\hline 29.5 & 995 & 492 & 150 & 1.00 & 1.00 & 100 & 1.00 & 1.00 & 0.95 & 0.75 & & \\
\hline 300 & 1012 & 0 & 0 & 100 & 1.00 & 1.00 & 1.00 & 1.00 & 1.00 & 0.75 & & \\
\hline & & & $-\mathrm{C}$ & 20 & 25 & 30 & 35 & 40 & 45 & 50 & 55 & 60 \\
\hline & & & $F$ & 68 & 77 & 86 & 85 & 104 & 113 & 122 & 131 & 140 \\
\hline
\end{tabular}

Temperature \& Altitude Derate 1. Deternine derate multipler vs. temperature and altitude in Table A or $B$ depending upon your operating condition.

2. Assumes the LT retum lemperature is $10 \operatorname{deg} \mathrm{C}$ above the air fitter inlet with a maximum LT lemperature of 45 deg $C$.

3. If the LT temperature exceeds 45 deg C, consult factory for recommendations.

4. Altitude is based upon SAE standard ambient pressure vs altude for low berometric conditions add $150 \mathrm{~m}$ ( 500 fi) to site altitude.

- Based on SAE standard ambiert pressure vs. altitude. Assumes LT retum temperatuat is $10 \mathrm{C}$ above air finter lniet.

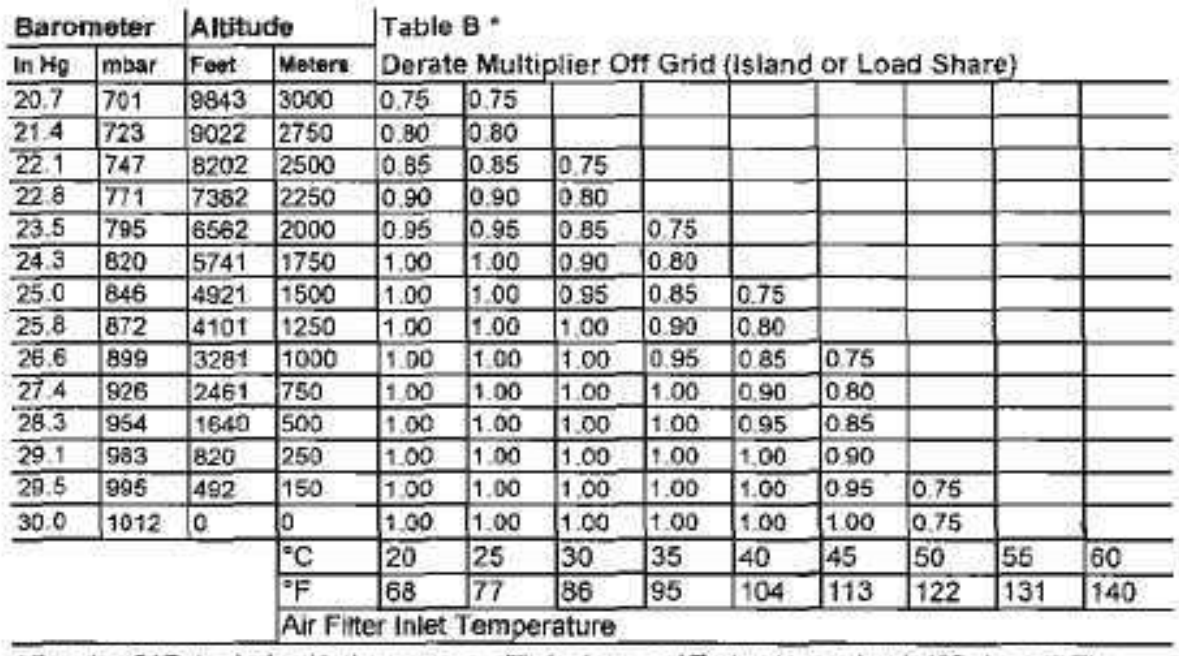

\begin{tabular}{|c|c|c|c|c|c|c|c|c|c|c|c|c|}
\hline \multicolumn{4}{|c|}{$\begin{array}{l}\text { Heat Rejection Fact } \\
\text { Barometer Altitude }\end{array}$} & \multirow{2}{*}{\multicolumn{9}{|c|}{$\begin{array}{l}\text { (altitude and ambient) for HT and LT Circuits } \\
\text { Table C } \\
\text { Multiplier for HT \& LT Heat Rejection vs Alt \& Temp. }\end{array}$}} \\
\hline \multirow{2}{*}{$\frac{\ln \mathrm{Hg}}{20.7}$} & \multirow{2}{*}{\begin{tabular}{|l|} 
mbar \\
701
\end{tabular}} & \multirow{2}{*}{\begin{tabular}{|l|} 
Feot \\
9043 \\
\end{tabular}} & \multirow{2}{*}{\begin{tabular}{|l|} 
Meters \\
3000 \\
\end{tabular}} & & & & & & & & & \\
\hline & & & & 1.11 & $1: 13$ & 1.14 & 1.15 & 1.17 & 1.18 & 1.19 & 1.20 & 1.22 \\
\hline 21.4 & 723 & 9022 & 2750 & 1.10 & 1.12 & 1.13 & 1.14 & 1.15 & 117 & 1.18 & 1.19 & 1.21 \\
\hline 221 & 747 & 8202 & 2500 & 1.09 & 1.10 & 1.12 & 1.13 & 1.14 & 1.16 & 1.17 & 1.18 & 1.20 \\
\hline 228 & 771 & 7382 & 2250 & 108 & 1.09 & 1,11 & 1.12 & 1.13 & 1.14 & 1.16 & 1.17 & 1.18 \\
\hline 23.3 & 795 & 6562 & 2000 & 1.07 & 1.08 & 100 & 1.11 & 1.12 & 113 & 1.15 & 1.16 & 1.17 \\
\hline 243 & 820 & 6741 & 1750 & 1.06 & 1.07 & 1.08 & 1.10 & 1.11 & 112 & 1.14 & 1.15 & 1.16 \\
\hline 250 & 846 & 4921 & 1500 & 1.05 & 1.06 & 1.07 & 1.09 & 1,10 & 111 & 1.12 & 1.14 & 1.15 \\
\hline 25.8 & 872 & 4101 & 1250 & 104 & 1.05 & 1.06 & 1.07 & 1.09 & 1.10 & 1.11 & 1.13 & 1.14 \\
\hline 26.6 & 899 & 3281 & 1000 & 1.02 & 1.04 & 1.05 & 1.06 & 1.08 & 1.09 & 1.10 & 1,12 & 1.13 \\
\hline 27.4 & $92 B$ & 2461 & 730 & 1.01 & 1.03 & 1.04 & 1.05 & 1.07 & 1.08 & 1.09 & 1.10 & 1.12 \\
\hline 28.3 & 954 & 1640 & 500 & 1.00 & 1.02 & 1.03 & 1.04 & 3.05 & 1.07 & 108 & 1.09 & 1.11 \\
\hline 29.1 & 963 & 320 & 250 & 1099 & 1.00 & 102 & 1.03 & 1.04 & 1,06 & 1.07 & 1.08 & 1.10 \\
\hline 29.5 & 995 & 492 & 150 & 0.99 & 1.00 & 1.01 & 1103 & 1.04 & 1.05 & 1.08 & 1.08 & 1,09 \\
\hline 30.0 & 1012 & 0 & 0 & 0.98 & 0.99 & 101 & 1.02 & 1.03 & 1.05 & 1.06 & 1.07 & 1.08 \\
\hline & & & ${ }^{\circ} \mathrm{C}$ & 20 & 25 & 30 & 35 & 40 & 45 & 50 & 55 & 60 \\
\hline & & & ${ }^{\circ} \mathrm{F}$ & 68 & 77 & 86 & 95 & 104 & 113 & 122 & 131 & 140 \\
\hline & & & Air: & 1 & Temp & ature & & & & & & \\
\hline
\end{tabular}

Methane Number Capability
Load (Percent of Rated)
\begin{tabular}{|c|c|c|c}
\hline $100 \%$ & $90 \%$ & $75 \%$ & $50 \%$ \\
\hline 78 & 72 & n/a & nia \\
\hline
\end{tabular}

LT \& HT Circuit Heat Rejection Calculation 1. Determine derate multiplier vs. temperature derate per above. 2. Using the multplier from \#1 above as the percenl load factor determine the Heal rejection fiom the previous pege.

3. From Table $C$ find the $H T$ and $L T$ circuit multiplifer.

4. Multiply the result of step 2 by the result of step 3 to obtain the heat rejection at your attitude and temperature 


\begin{tabular}{|c|c|c|c|c|c|c|c|}
\hline \multicolumn{8}{|c|}{ Alternator Data } \\
\hline Voltage Range & $\begin{array}{l}\text { Connection } \\
\text { Configuration }\end{array}$ & $\begin{array}{l}\text { Temp Rise } \\
\text { Degrees C }\end{array}$ & $\begin{array}{l}\text { Duty }^{11} \\
\text { Cycle }\end{array}$ & $\begin{array}{c}\text { Single Phase } \\
\text { Factor }\end{array}$ & $\begin{array}{c}\text { Maximum } \\
\text { Surge kVA }\end{array}$ & $\begin{array}{l}\text { Alternator } \\
\text { Data Sheet }\end{array}$ & $\begin{array}{c}\text { Feature } \\
\text { Code }\end{array}$ \\
\hline 380 & Wye, 3 Phase & 105 & C & N/A & 7960 & 515 & $8597-2$ \\
\hline $416-480$ & Wye, 3 Phase & 80 & C & N/A & 9700 & 517 & B587-2 \\
\hline $416-480$ & Wye, 3 Phase & 105 & C & NIA & 8400 & 516 & 86542 \\
\hline $416-480$ & Wye, 3 Phase & 125 & C & N/A & 7200 & 515 & $8627-2$ \\
\hline 480 & Wye, 3 Phase & 80 & C & N/A & 8400 & 516 & $8653-2$ \\
\hline 480 & Wye, 3 Phase & 105 & C & NIA & 7200 & 515 & B583-2 \\
\hline$\overline{600}$ & Wye, 3 Phase & 80 & $\mathrm{C}$ & NIA & 8250 & 516 & B589-2 \\
\hline 600 & Wye, 3 Phase & 105 & C & N/A & 7200 & 515 & $B 582-2$ \\
\hline 4160 & Wye, 3 Phase & 80 & C & N/A & 6300 & 518 & B590-2 \\
\hline $12470-13800$ & Wye, 3 Phase & 80 & C & N/A & 8000 & 523 & B591-2 \\
\hline $12470-13800$ & Wye, 3 Phase & 105 & C & $N / A$ & 6800 & 522 & B484-2 \\
\hline $13200-13800$ & Wye, 3 Phase & 105 & C & N/A & 5000 & 521 & B657-2 \\
\hline 13800 & Wye, 3 Phase & 80 & C & N/A & 6800 & 522 & B565-2 \\
\hline & & & & & & & \\
\hline & & & & & & & \\
\hline
\end{tabular}

\title{
Continuous Rating Definition
}

\begin{abstract}
Applicable for supplying power continuously to a constant load up to the full output rating for unlimited hours. No sustained overload capability is available for this rating. Consult authorized distributor for rating. (Equivalent to Continuous Power in accordance with iSO8528, ISO3046, AS2789, DIN6271, and BSE514). This rating is not applicable to all generator set models.
\end{abstract}

Notes

1) Woights and set dimesions represent a generater set with its standard features onty. See outline drawing for other configurations.

2) At iSO3046 reterenco conditons, aftrude 1013 mbar $\left(30 \mathrm{in} \mathrm{Hg}\right.$ ) air inlet temperature $25 \mathrm{C}\left(7 T^{\prime} \mathrm{F}\right)$

3) Nominal performance $\pm 21 / 2 \%$

4) According to iSO $3046 /$ with fuel consumption tolerance of $+5 \%-0 \%$ or efficiency tolerance of $+0 \%-5 \%$

5) Production variation/toierance $\pm 5 \%$.

8) At eloctical output of 1.0 Power Factor.

7) Tested using pipeline natural gas with LHV of $33.44 \mathrm{~mJ} / \mathrm{Nm}^{3}$ (9058TUMt3)

8) Outiet vemperature controlied by themostat. Intet temperature for referance only.

9) iniet temperature controined by themostax, outbet tamperature for roference only.

10) Wha engine dnven coolant purnp.

11) Standby (S). Prime (P), Contiauous (C)

12) Maximum rated starting kVA that results in minisnum of $\$ 0 \%$ of rabed sustained voltace during starting.

13) Tolerance $+1-15 \%$

14) Exhaust systom back pressure is a ratad loed and wili decrease at lower loads.

Curmmins Power Generation $140073^{\text {Ti }}$ Avenue NE

Minneapolis, MN 55432 USA

Telephone: 7635745000

Fax: 7635745298

Web: www.cumminspower com
Cummins Power Generation Manston Park, Columbus Avenue Mansion. Ramsgate

Kent CT 12 5BF, UK

Telephone: +44 (0) $1843-255000$

Fax $+44(0) 1843-255902$

Email cpq uk@cummins com

Web: www cumminspower com
Cummins Power Generation

8 Tanjong Penjuru

Singapore 609019

Telephone: +65 265-0155

Fax $+65264-0664$ or $265-6909$

Email mktg(t)sing cummins com

Web: www cumminspower com

\section{Power Generation}


Genset-Spark Ignited, $60 \mathrm{~Hz}, 2000$ kW

Continuous Rating

Fuel System-PipeNatGAS

Emissions-Exhaust

Thermostat-Engine Cooling

Voltage 480 vac

SetCntl-PwrComm Supervisor, ShipLoose

Display Language-English

User Interface-Control,Graphical

Interface-CommunicationskletworkGCP

Temp Sens-Stator,2RTD/Ph

Temp Sens-Bearing RTDs

Transformer-Bus Potential $240 \mathrm{~V}$

AuxSupplyolts 480,3Ph, 4W, 120V1 Ph2W

Heater-Alternator,WThermostat

Heater-Alternator, WThermostat

Cooling Control Module - W/ Engine Pumps

Coolant Connections-Flanged (ASA Flanges)

Oil Heate r-40F MinAmbient Temp

Engine Air Cleaner - Heavy Duty

ExhaustConnector-ASA. Flange

Crankcase Breather Filter

Oil Sump Makeup Valve

Lite rature-English

Natural Gas Fuel Train

Packing-None

* Estimate

* Estimate

*Estimate
Freight to USA Port

Duty into US

Freight port to jobsite

Vibration Isolators

GCP Calbe and Harness 5 meters 


\section{Cummins vigital raraileing Low Voltage Proposal Summary}

Job Nime: Northen Wichigan C oyen

Cumrnins Distriluror: Cummins Brilgeway

A dithess:

Oactation ID:

Propesal 1er: Buce onte

Corri ol Type:

PCUL300, 2.3 Gensets contr olled. 1 to 2 Load Sheds, Generator Data Di spiay. Exdended

Paraseling thisty Tracking Featule

System Volhage: $277.480 \mathrm{~N}, 39.4$-wire

Section Deptix 78 inches

Section heoht: 90 inches

Approx. total theup lensth: 12.5 teet

Appeximate weight: 4800 Lbs

Other Features:

Wanuals, Freight, Start-up,

Standard internuting Ratings

All breakers are Square D Masterpact type.

Section Detall

$\begin{array}{lll}1 & \text { Utility Main } & \text { Breaker Size(s); } 4000 \text { amps, } 1 \text { Ground Fautt(s) } \\ \text { EOO-L, S, I, Relays 86, 81-O/N, 47 R7/59, 32R, O/C Lamps, PT's \& CT's, Aux Switch }\end{array}$

2 Distribution
$\mathrm{MO}-\mathrm{L}, \mathrm{S}, \mathrm{I}$$\quad$ Breaker Size(s): , . .4000 amps, 1 Ground Fault(s)

3 Generator Paralleling Breaker Size(s); , 3000, , 3000 amps, 2 Ground Fault's)

EO - L, S, I, PT's \& CT's. Aux Switch

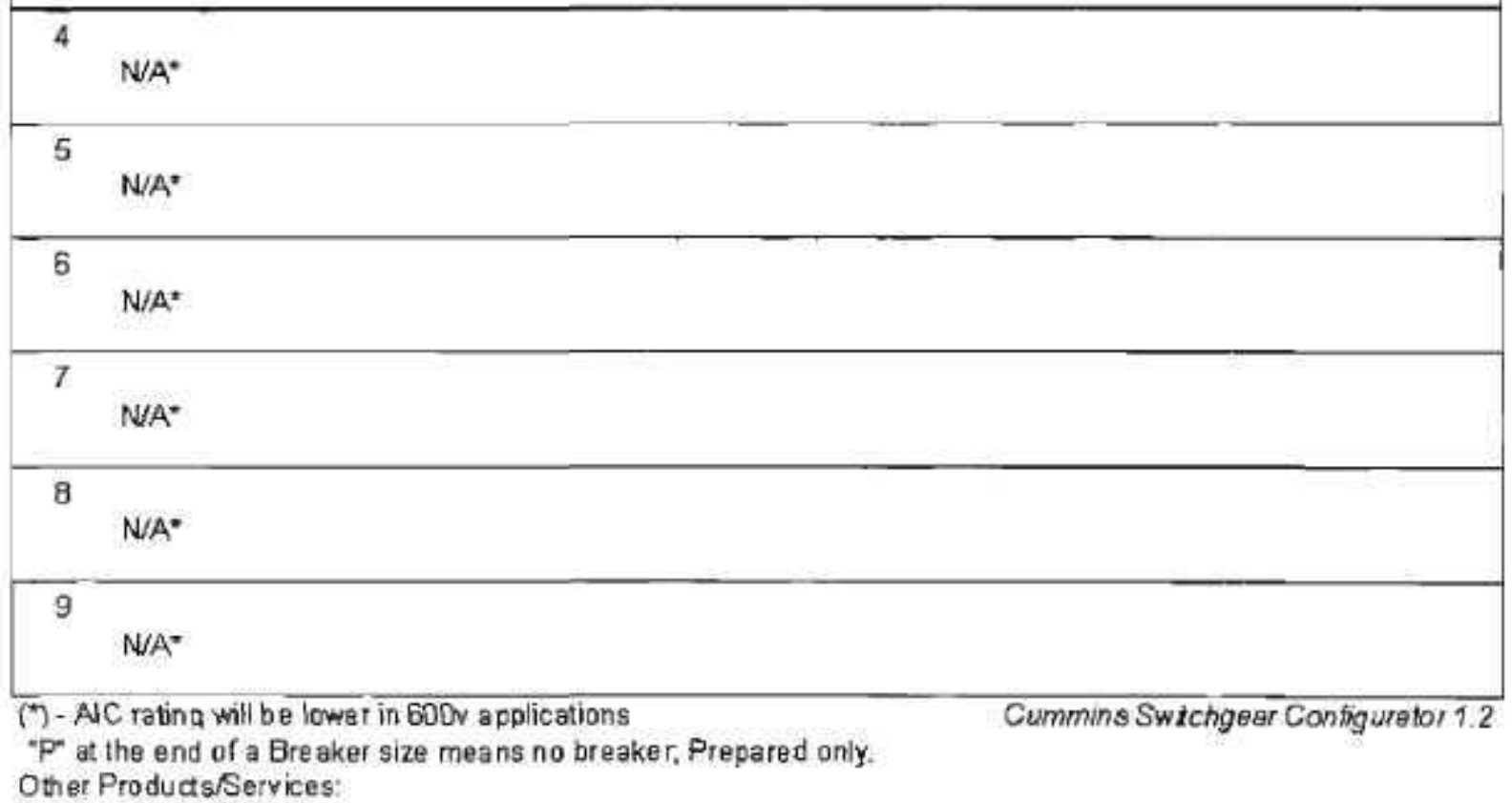


Appendix 3. Phase One Screening Report - Environmental 


\title{
Phase One Screening Report:
}

\section{Saginaw Chippewa Windpower Sites}

\author{
Arenac and Isabella Counties, Michigan
}

March 18, 2005

Prepared for:

Distributed Generation Systems, inc (DISGEN) 200 Union Blvd, Suite 304

Lakewood, Colorado 80228

On Behalf of:

Saginaw Chippewa Indian Tribe

770 East Broadway

Mount Pleasant, Michigan 48858

Prepared by:

Rhett E. Good

Western EcoSystems Technology, Inc.

2003 Central Avenue Cheyenne, Wyoming 82001

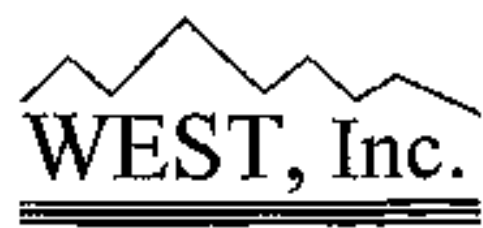




\section{INTRODUCTION:}

When exploring prospective windpower sites, knowledge of wildlife and other biological resource issues helps the wind industry identify and avoid potential ecological problems early in the development process. The purpose of this report is not to define impacts of the proposed windpower project, rather, the purpose is to alett project proponents to potential conflicts with wildlife and habitat. WEST, Inc. was asked by DISGEN to evaluate potential wildlife occurrence and habitat issues at two prospective windpower siles for the Saginaw Clippewa Nation in Michigan. The areas are located within Arenac and Isabella Counties near Mount Pleasant and Standish, Michigan (Figure 1). The area evaluated for potential biological resources includes the proposed project areas, additional potential development areas, and a two nile buffer. This report focuses on the following potential areas of concem:

- Raptors

1. Identify areas of potentially high nesting density

2. Identify areas of potentially high prey density

3. Examine topography to determine the potential for high use and potential nest locations

4. Determine the species likely to occur in the area

5. Determine the potential for migratory pathways

- Candidate, Proposed, Threatened, Endangened, and USFWS Birds of Conservation Concem

1. Identify the potential occursence of federally listcd ot state protected species through existing literature and database searcles

2. Evaluate the suitability of habitat at the wind plant site for protected species

- State Wildlife Issues (using existing state wildlife agency information)

1. Examine habitat during site visits to determine the potential for use by state protected species

- Unique Habitat

1. Evaluate the uniqueness of the site relative to the surrounding area.

- Wetlands

1. Deternaine the potential for wetlands at the site through a cursory site visit and examination of available data

- Bats

1. Determine the proxinity to potential feeding sites and hibernacula

2. Determine species likely to occur in the area

- Avian Migratory Pathways of Passerines, Waterfowl and Shorebirds 


\section{METHODS:}

Biological resources within the vicinity of the project were evaluated through a search of existing data and a site visit. The project areas were examined on December 20, 2004 by foot and vehicle. During the site visit, biological features and potential wildiffe habitat including plant communities, topography features, and potential raptor nest structures were identified.

Several sources of available data were used to identify biological resolirces within the project areas, including requesting data from the Michigan Department of Natural Resources, the Michigan U.S. Fish and Widlife Service (LSFWS), Michigan Natural Features Inventory, and searching published literature, field guides etc. Letters requesting information on the proposed projects were sent to the USFWS and the Michigan Department of Natural Resources (DNR) on Febrnary 9, 2005 and December 30, 2004 respectively (Appendix A). Comespondence was received from the Michigan DNR and the U.S. Fish and Wildlife Service dated February 24, 2005.

After biological resources within the project areas were identified, we analyzed the potential for conflicts with the proposed windpower project based, in part, upon studies conducted at other wind plants throughout the U.S. We also calculated Potential Impact Scores based on the Interim USFWS guidelines for the proposed project areas and one reference area (See Appendix $B$ for score calculations).

Study Area. The two project areas are located within central and east-central Michigan. Both project areas are a mixture of private and Saginaw Chippewa lands. The project areas consist of active and formerly tilled agriculture and seattered woodiols composed of Box Elder (Acer negundo) and Eastern Cottonwood (Populus delloides). Scattered nural residences are present within botl areas. The Mount Pleasant site is located approximately two miles east of Mount Pleasant. Michigan in Isabella County (Figure 2). The Standish site located approximately four miles southeast of the town of Standish and within one mile of Saginaw Bay (Figure 3). The Saganing River flows through the Standish Project Area to the Saginaw Bay.

\section{RESULTS:}

\section{Raptor Issues}

Nesting density and species breeding in area. Nesting habitat in both project areas for above ground nesting raptors is limited to deciduous forest. Patches of woodlots are present within two miles of both project areas. Based upon available habitat, the following species are most likely to nest within two miles of the project areas (Chartier and Ziamo 2004): ted-tailed hawk (Buteo jamaicensis), red-shouldered hawk (Buteo lineafus), broad-winged hawk (Buteo platypterus), American kestrel (Falco sparverius), Cooper's hawk (Accipiter cooperii) and sharp-shinticd hawk (Accipiter striatus). Due to the presence of Saginaw Bay near the Standish site, the potential exists for osprey 
(Pandion haliaetus) to nest within two miles of the project area. Although the bald eagle (Haliaeetus leucocephatus) has been documented as nesting on \$aginaw Bay, the proposed Standish project area generally lack mature forests required for bald eagle nests, and the potential for the species to nest in the project areas is considered low. The potential for bald eagles nesting within the potential development area is greater due to the closer proximity to the shoreline of the Saginaw Bay.

Nesting raptors are generally territorial and do not nest in high densities unless certain factors are limiting, such as nesting habitat. Scattered woodlots are present throughout Isabella and Arenac Counties, and there is no available evidence to suggest that habitat within the project areas support extraordinary high densities of nesting raptors.

The proposed project areas also provide habitat for wintering raptors. Raptor species most likely to winter in the project areas include northern harrier (Circts cyanteus), sharpshinned hawk, Cooper's hawk, red-tailed hawk, nough-legged hawk (Buteo lagopus), and American kestrel (Chartier and Ziann 2004). A lower potential exísts for gytfaIcon (Falco rusticolus), northem goshawk (Accipiter genfilis), and great gray owl (Strix nebulosa) to utilize the project areas.

Potential for prey densities. No obvious signs of colonial rodents were observed due to the preponderance of tilled agriculture in the project area. Prey densities and prey availability of species such as Peromyscus may be very high in agricultural fields immediately afler harvest as mice forage on leftover seeds.

Overall, it is very difficult to assess potential prey densifies during a single site visit. Prey densities can thuctuate rapidly based on habitat and climatic factors. However, overall prey densities are expected to be low in the project area based on the large amount of tilled agriculture in the area.

Does the topography of the site increase the petential for raptor use? The proposed project is located on almost entirely flat agricultural fields that gereratly lack defined topographic edges. At other windpower facilities located on prominent ridges with defined edges (e.g., rims of canyons, steep slopes), raptors fly along the sim edges, using updrafts to maintain altitude while hunting, migrating or soaring. Turbines are often placed on prominent ridges in order to use higher wind speeds and updrafts that raptors also use. In Wyoming, raptors most often used areas within $50 \mathrm{~m}$ of the rim edge (Johnson et al. 2000b). Raptor use is not expected to be heavily infleenced by the topogtaphy in the project area because its general lack of defined ridges and rim edges. However, due to the presence of the Saginaw Bay near the Standish site, there is the potential for increased use of the Standish site by migtating raptors. Raptor species generally avoid migrating over large bodies of water (Kerlinger 1995). 


\section{Federal and State Profected Species}

A total of 82 federally or state listed threatened or endangered species and 133 species of concern occur within the state of Michigan (MNFI 1999). Most species groups are included, such as birds, mammals, ampłibans, reptiles, mussels, fish and various types of insects. The vast majority of these species occur primarily in non-agriculturat native habitats and streams or lakes. The proposed project areas occur primarily within habitats that are actively or fomerly tilled, thus there is limited potential for most listed species or species of concern to occur within tlye project areas. The potential development areas containing greater amounts of native habitat in the form of deciduous woodlands and wetlands, thus there is a greater potential for species to occur within the potential development areas and two mile buffers. The MNFI has records for only two species occurring within approximately two miles of either project area. The wood turtle and the Blanding's turtle were documented within two miles of the Mount Pleasant Project area (Figure 2). The MNEt also has records for Hayden's Sedge (extirpated), Ram's head lady slipper, and riverine snaketail occurring within arcas surrounding the Mount Pleasant project. In correspondence dated Februaly 24, 2004, the U.S. Fish and Wildlife Service determined "there are presently no federally-listed threatened, endangered or proposed species within the project area."

MNFI data should be interpreted with caution. Surveys have not been conducted in many areas of Michigan, and the absence of records does not necessarily indicate a species does not occur. Due to the lack of records in many ateas, we examined records of species occurring within Isabella and Arenac Counties to deternine the potential for other listed species or species of concem to occur within the proposed project and development areas (Tables 1-2).

Table 1. A list of species tracked by the MNFI with records in Isabella County. The list is corrent as of Jautary 4, 2005, and contains all plant and animal species except birds. $\mathbf{E}=$ Eudangered, $\mathbf{T}=$ Threatened, $\mathrm{SC}=$ Species of Concern, and $\mathrm{X}=$ Extirpated.

\begin{tabular}{|c|c|c|c|c|}
\hline Common Name & $\begin{array}{l}\text { Feterat } \\
\text { Status }\end{array}$ & $\begin{array}{l}\text { State } \\
\text { Staturs }\end{array}$ & $\begin{array}{l}\text { Potentially Present } \\
\text { at Mt Plesaut? }\end{array}$ & Notes \\
\hline Calypso or Fairy-slipper & & $T$ & No & Lacks $\mathrm{Habitat}$ \\
\hline Hayden's Sedge & & $\mathrm{X}$ & No & Extirpated \\
\hline Ram's Head Lady's-slipper & & $\overrightarrow{\mathrm{SC}}$ & No & Lacks Coniferous Habitat \\
\hline Blanding's Turtle & & SC & Yes & $\begin{array}{l}\text { Records within } 2 \text { miles of } \\
\text { project }\end{array}$ \\
\hline Wood Tutle & & $\mathrm{SC}$ & Yes & $\begin{array}{l}\text { Records witbin } 2 \text { miles of } \\
\text { project }\end{array}$ \\
\hline Splendid Chubtail & & $\$ C$ & Yes & $\begin{array}{l}\text { Wetland habitat present near } \\
\text { project area }\end{array}$ \\
\hline Rapids Clubtail & & $\overline{\mathrm{SC}}$ & Yes & $\begin{array}{l}\text { Wetland habifat present near } \\
\text { project area }\end{array}$ \\
\hline Twinleaf & & $\mathrm{SC}$ & $\overline{\text { Yes }}$ & $\begin{array}{l}\text { Potential to occur in forested } \\
\text { areas }\end{array}$ \\
\hline Blue-yed-grass & & SC & No & Lack of Habitat \\
\hline Eastem Box Turtle & & $\mathrm{SC}$ & Yes & Suitable Habitat Present \\
\hline
\end{tabular}


T'able 2. A list of specles tracked by the MNNT with records in Areaac Connty. The list is carrent as of Jannery 4, 2005, and contains all plant and antmal species except birds. $E=$ Endangered, $T=$ Threatened, SC = Species of Concern, and $\mathrm{X}=$ Extirpaled.

\begin{tabular}{|c|c|c|c|c|}
\hline Comman Name & $\begin{array}{l}\text { Federal } \\
\text { Statess } \\
\end{array}$ & $\begin{array}{l}\text { State } \\
\text { Status }\end{array}$ & $\begin{array}{l}\text { Potentially Present } \\
\text { at Standish? }\end{array}$ & Notes \\
\hline Pitcher's Thistle & $\mathrm{LT}$ & $\tau$ & Yes & Only in sandy areas on lakeshore \\
\hline Large Toothwort & & $T$ & Yes & In Mesic forested areas \\
\hline Wood Turtle & & $\mathrm{SC}$ & Yes & Near streatus in project area \\
\hline Dotl's Merolonche & & $\mathrm{SC}$ & Yes & May fly through project areo \\
\hline $\begin{array}{l}\text { American Butying } \\
\text { Beetle }\end{array}$ & $\mathrm{LE}$ & $E$ & No & $\begin{array}{l}\text { The USFWS does not list as } \\
\text { potentially occunting in Arenas } \\
\text { County }\end{array}$ \\
\hline Channel Dartet & & $\mathrm{E}$ & Yes & In streams \\
\hline $\begin{array}{l}\text { Eastern } \\
\text { Massasauga }\end{array}$ & $C$ & $\mathrm{SC}$ & Yes & Tn wetlands \\
\hline
\end{tabular}

Because windpower projects typically pose a greater risk to birds and bats than other species, we examined the potential for all listed and species of concern of birds to oceur within the project areas (Tables 3-4). Only two bat species, the Indiana bat (Endangered) and the eastem pipistrcile (Spectes of Concern) are listed by the MNFI as mammals of special concern in the state of Michigan. The proposed project areas occur outside of the potential range of these two species.

\section{WETLANDS}

The number of wetlands differs between the two project areas (Figures 2-3). Forested wetlands are present within and surtounding the Mount Pleasant potential development area (Figure 2). Wetlands present within the Standish project area are associated with the Saganing River. The Saginaw Chippewa Nation has designated a nature preserve located on the Saganing River that is composed largely of wetlands. Within two miles of the Standish site several emergent wetlands are present along Saginaw Bay. Some forested and scrub-shrub wetland are present within woodlots west of the proposed project area (Figure 3).

\section{STATE WILDLIFE ISSUES AND UNIQUE HABITAT}

Based on correspondence from the Michigan DNR, no natural features should be impacted by the proposed project (see attached correspondence). The DNR's largest concern was the potential impact of the proposed project on migrating bitds. The DNR describes Michigan's flyways as concentrated along the Great Lakes shorelines.

Both project areas are dominated by agiculture and have some woodlots present (Figures 4-5). The potential development area for Mount Pleasant contains more deciduous forest 
and forested wetlands. These habitats, while not unique in Central Michigan, provide more suitable habitat for several native bird and mammal species than areas dominated by agticulture.

\section{BATS}

The proposed project areas occur within the potential ranges of the following bat species (BCI 2002): Big brown bat (Eptesicus fuscus), eastotn red bat (Lasiurus borealis), hoary bat (Lasiturus cinereus), silver-haired bat (Lasionycteris noctivagans), little brown myotis (Myotis lucifugus) and northern myotis (Myotis septentrionalis). Both proposed project and potential development areas contain potential roosting and foraging habitat for bats in the form of woodlots and wetlands.

Bat casualties have been reported from most windpower facilties where post-construction fatality data are publicly avajlable. Reported estimates of bat mortality at windpower facilities have ranged from $0.01-47.5$ per turbine per year $(0.9-43.2$ bats $/ \mathrm{MW} /$ Year) in the U.S. with an average of 3.4 per turbine or 4.6 per MW (NWCC 2004). Most of the bat casualties at windpower facilities to date are migratory species which conduct long migrations between summer roosts and winter hibernacula. Examples of these species commonly found as fatalities at windpower facilities include hoary bats, silver-haited bats and eastern red bats. A recent teport of bat fatalities at a windpower facility in West Virginia includes relatively high numbers of red bats, hoary bats, eastern pipistrelle (Pipistrellus subflavis) and little brown bat over the course of one year. The West Virginia site is located on a prominent, relatively narrow ridge in the Appalachian Mountains and is surrounded almost entirely by deciduous forest. The causes of the relatively high number of migratory bat deaths at windpower facilities are not well understood. Some have suggested it may be related to the lack or reduction of echolocation during migration (Johnson 2003). Futhermore, strong field methods to provide quantitative predictions of migratory bat use are lacking.

Due to a lack of information concerning bat migration habits, it is difficult to predict if the proposed project areas are located within a bat migration corridor. The proposed project will likely result in the mortality of some bat species, including red bats, boary bats and silver-haired bats. The magnitude of these fatalities and the degree to which other bats species will be affected is difficult to determine. However, trilike the West Virginia site, the proposed project areas are relatively flat and do not appear to contain topographic features that may funnel migrating bats (Figures 1-2). 


\section{AVIAN MIGRATORY PATHWAYS}

The seasonal migration of birds through Michigan appears to be generally widespread thoughout Michigan. However, radar omithology research focusing on noctumal songbird migration in this region suggested that the shorelines of the Great Lakes may be important stopover sites (Diehl et al. 2003). The Great Lake's shorelines and peniusulas are also believed to concentrate the migration of raptors (Kerlinger 1989, Kerlinger 1995).

Although night-migrating songbirds typically migrate between 91 and $610 \mathrm{~m}$ altitude, they occasionally collide with lit communication towers and other tall humatn-mate stractures (Erickson et al. 2001, Kerlinger 1995). Research suggests that birds become attracted to the lights of structures and fly around them in circular patterns, eventually beconing exhausted, and/or colliding with the stucture, supporting guy wires or other birds (Avery et al. 1976, Larkin and Frase 1988). Bird collisions with tall structures, including communication towers, appear to occur more frequently on nights with overcast skies, fog, or otherwise low-visibility. Similar to commurication towers, many wind turbines are equipped with obstruction lighting. Typically turbines are only lit with one flashing red or white strobe light on the turbine nacelle compared to the flashing light - steady burning light combinations of communication towers (FAA 2000). Although the turbine lighting systems may attract some night-migrating songbirds, mortality at turbines is significantly lower than what has been documented at lit communication towers. Bird mortality eveuts involving hendreds of birds have occurred at communication towers, but no large mortality events have ever been documented at wind turbines (Erickson et al. 2001 ).

The proposed Standish project area is located within one mile of the shore of Saginaw Bay. In general, the proximity of the site to the shoreline could be associated with higher densities of both nocturnal and diurnal migrating birds. Although the proposed project area provides very little of the vegetation necessary for songbird stopovers, waterfowl may use the agricultural fields for feeding. Areas managed for breeding Kirtland's Warbler (Dendroica kirtlandii), are present 31 miles north of the proposed turbine site. Although the potential for a Kirtland's warbler to utilize deciduous forest in the project area for stopover habitat is considered low, the potential exists and should be recognized.

The proposed project area near Mount Pleasant, Michigan had been used for agricultural purposes in the past but has since become failow, and is currently fominated by grass and other herbaceous vegetation. East and northeast of the proposed turbine site is a shelterbelt and wooded areas that are contiguous with the Au Sable State Forest (i.e., State Forest boundary 4 miles east of site). According to the National Wetlands Inventory much of the forested areas near the proposed turbine sites are wet, and Onion Creek flows due south and east of the site. Although this site is significantly farther from 
the shoreline than the Standish site, it is possible that migratory birds may use the area as stopover habitat.

\section{DISCUSSION:}

The largest issue facing both proposed project areas is the potential jmpact to migrating birds, especially noctumal migrants. To date overall fatality rates for birds at windpower projects have ranged from $0.6-7.7$ bird fatalities / turbine $/$ year $(0.9-11.7 / \mathrm{MW} /$ year), with an average of 2.3 bird fatalities / turbine / year or 3.1 bird fatalities / MW/ year (NWCC 2004). Outside of California, fatality rates for raptors have ranged from 0.0 -0.07 fatalities / tarbine / year $(0.00-0.09 / \mathrm{MW} /$ year $)$, with an average of $0.03 \mathrm{per}$ turbine and 0.04 per MW. The largest single fatality event at a windpower project was 30 songbirds on a foggy night near a lighted substation in West Virginia (NWCC 2004). Bird mortality at turbines in the eastem United States is typically much lower than what is expected at communication towers. Ongoing bird collision research in Michigan has found higher bird mottality at communication towers $>305 \mathrm{~m}$ Above Ground Level (AGL) than at towers $<146 \mathrm{~m}$ AGL (Geluring 2005). It is possible that bird mortality may increase as turbine height increases.

The proposed projects may potentially occur within the migratory pathway of the Endangered Kirtland's warbler. The U.S. Fish and Wildlife Service places a high priority on protecting this species, and the potential exists for individuals of this species to collide with the proposed turbines in the project area. It is very difficult to evaluate the level of risk due to the lack of understanding of specific migratory pathways in the proposed project areas. Assuning bird migration is concentrated along the Great Lakes shoreline, the Standish project area may pose a greater risk to migrating Kirtland's warblers than the Mount Pleasant project area. While the overall risk to the Kirtland's warbler is considered low due to 1 ) the low songbird mortality observed at other windpower projects and 2) the expected small size of the project, the potential exists for individuals of the species to collide with turbines in the project area and should be realized. It should also be roted that other existing tall structures in central Michigan, such as tall buildings and communication towers pose potentially greater threats to migrating Kirtland's warblers.

The presence of Saginaw Bay within one mile of the proposed Standish site causes a greater potential for biological issues versus the Mount Pleasant Site (Tables \$-6 and Appendix B). This is primarily due to the potential for migrating songbirds and raptors to more heavily utilize areas along shorelines for stopover habitat as well as for potential migration routes. Little is known conceming bat migrations, and it is not clear if bats avoid migrating over large bodies of water and thus also concentrate along shorelines.

The proposed project areas are dominated by active and former agricuiture, and provide limited habitat value to wildlife species. The potential development areas surrounding each project area contain greater amounts of natjve habitats, such as deciduous forest and 
wetlands. If the proposed project impacts these native habitats, the potential exists for species protected under the Micligan Endangered Species Act to occur within these liabitats. If the proposed project moves forward, the MI DNR and the USFWS should be consulted again once turbine and other infrastructure locations have been decided to determine the noed for bjological surveys. 


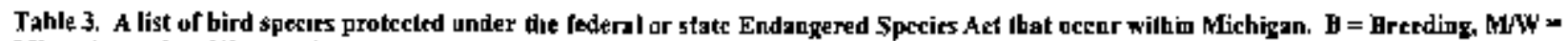

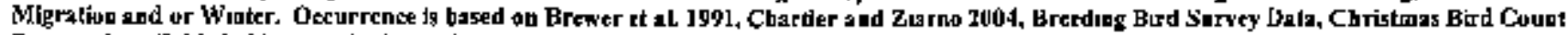
Dais, and avaliable babilut prithis the jroject areas.

\begin{tabular}{|c|c|c|c|c|c|}
\hline Commor Nerme & $\begin{array}{l}\text { Federal } \\
\text { Stetur }\end{array}$ & $\begin{array}{l}\text { Stptc } \\
\text { Sitntas }\end{array}$ & $\begin{array}{l}\text { Potebtully Prejent in } \\
\text { Mi Pleisunit tB. } \\
\text { MNW or Bothi }\end{array}$ & $\begin{array}{l}\text { Poleniully Preseal } \\
\text { Ia Standisb? (B, } \\
\text { MAW or Boch) }\end{array}$ & Nolex an Oecerreace \\
\hline S5ort-ersed oul] & & $\mathrm{J}$ & 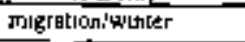 & minationiswanter & \\
\hline Pipune phovtr & LE & $\mathrm{E}$ & Potencially stigralonn & 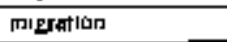 & 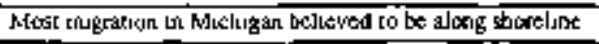 \\
\hline Ptairs warbtet & & $\mathrm{E}$ & Poienitally Mugтation. & Potentinly migralycon & \\
\hline K'vland's warblet & I. $E$ & $\mathrm{E}$ & Migratión & migatuon & 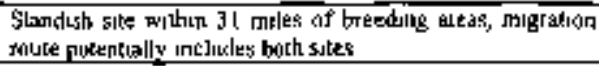 \\
\hline Percegulue talcon & & $\mathrm{E}$ & 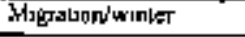 & Migration'wantes & \\
\hline Mıgr and1 loggethe-ad shutkc & & $E$ & Migrabunhwunter & Mugrariou'sustét & \\
\hline King ral & & $E$ & Nol lekely & Nor Jjkely & \\
\hline Eam $\sigma w \mid$ & & $\mathbf{E}$ & Not lukety & Not Jakely & \\
\hline Perstow's apartons & & $\mathrm{T}$ & Both & Bolk & 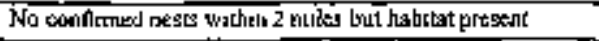 \\
\hline Tong-ared owi & & $\mathrm{T}$ & Mipratrovingerter & Mugratiobrunter & \\
\hline Rędrscivi_dered bawk. & & $7^{\prime}$ & Mugaskariwalet & Migrabouvwhiter & 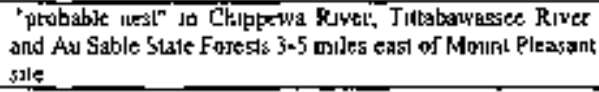 \\
\hline Yellow roll & & $\mathrm{T}$ & Nix likety & Nंar ]rkely & \\
\hline Trtuthigttęr sman & & $\overline{\mathrm{T}}$ & 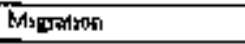 & niggration & \\
\hline Yaljort-Lhoiled wistble & & $\mathrm{T}$ & Non lukely & Nor Jıkely & \\
\hline Martın & & $T$ & Mupraikashougler & Mugratiog'wunter & \\
\hline Corturua lock & & $T$ & Magraixon'wonnter & Mdigralion'swunter & \\
\hline Binld esple & PS LT,Pח\}, & $\mathrm{T}$ & Mugarkov'silfitest & Mugrationiwusites & 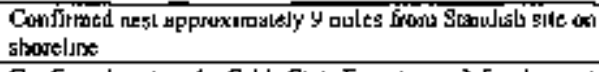 \\
\hline Ltâst bictern & & $\mathbf{T}$ & Mugraduns & 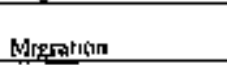 & 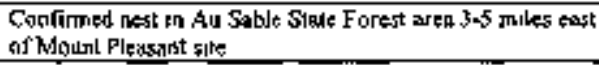 \\
\hline Ospery & & $\mathrm{T}$ & Mapralpobrinter & Migraboniwented & $\begin{array}{l}\text { Niest apgrosintately } 8 \text { wisles southwest of thount Pleasant } \\
\text { site }\end{array}$ \\
\hline Casplar 1tMr & & $\mathbf{T}$ & Migratiols & Mizsuncm & \\
\hline Conmon test & & $\mathbf{T}$ & Migratian & Mignxtact & \\
\hline Lart: apenrow & & $x$ & Not Lkely & Not likely & \\
\hline
\end{tabular}




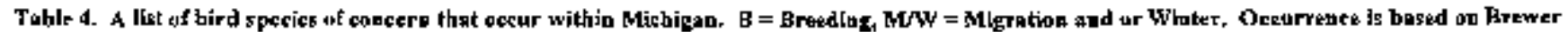

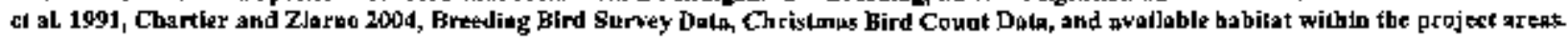

\begin{tabular}{|c|c|c|c|c|c|}
\hline Common Napa & $\begin{array}{l}\text { Fediral } \\
\text { Sentins }\end{array}$ & Slate Statud & $\begin{array}{l}\text { Potontlally l'reent in Stil } \\
\text { Plensant? (B, MUt' or Both) }\end{array}$ & 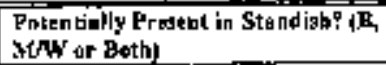 & Noted on Oecurrence \\
\hline Cooper's basph & & St: & Both & Both & 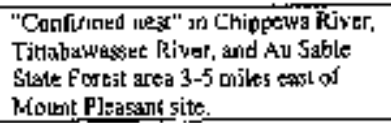 \\
\hline Nortberm goshowk & & SC & Migrztion'winter & Mtigrationd/wader & \\
\hline Ḡpsshopper spख्य0\% & $\overline{\mathrm{PS}}$ & $\mathrm{sc}$ & bolis & Batb & $\begin{array}{l}\text { "Possible nust" apploxudately } 6 \text { miles } \\
\text { gouthwest of Woulut Pleasant stit }\end{array}$ \\
\hline Япегтип bittem & & $5 C$ & Miærabon'winter & Migrralioubiriveter & $\begin{array}{l}\text { Probabje pes in Au Sable Sraic Forest } \\
\text { prea } 3-5 \text { miles east of Wkilurr Pleasant } \\
\text { gitc. }\end{array}$ \\
\hline Black tem & & SC. & Mtizration & Migration & \\
\hline Northern hasrett & & $\mathrm{SC}$ & Mtiveration'winter & Migratiols'wister & \\
\hline Marst hren & & SC & 3tigradion/wunter & rigration & \\
\hline Cenutan werblex & & SC & Nac likety, poxsbly ruxdion. & Not Jikkely, possibly mizratwió & \\
\hline Sptuce grtuse & & 논 & NoH Ijkely & Nos lakkely & \\
\hline Cabuntét nwotheo & & $\mathrm{Sc}$ & 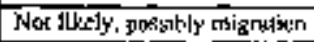 & Ňnat Jakely & \\
\hline Black-crowned alght-heron & & $3 C$ & eniglation'winter & migulion/winlet & \\
\hline Wilen's phatarope & & $\overline{\mathrm{SC}}$ & possibly mlgrabon & possibly sugeraron & \\
\hline Black-backed woodpecker & & SC & mierstion & milgration & \\
\hline Prothonotnזy ü:्यb]er & & $\mathrm{SC}$ & Not [izety & Nac Jikiedy & $\overline{5}$ \\
\hline Lovasiana prakerlhustsh & & $\mathrm{SC}$ & Nex Jikely & Shot litely & \\
\hline Dickcjesel & & SC & migration & Jilgridon & \\
\hline Fotster's Ierr & & SC & Wot likely & Possithly niggratwa & \\
\hline 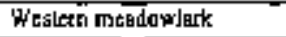 & & SC & possib|y migration & Not jakty & \\
\hline Sherp-bided groke & & $\overline{S C}$ & Mat idkedy & No4 likely & \\
\hline$\overline{\text { H}} \infty \mathrm{d} \overline{\mathrm{ed}}$ wartier & & $\overrightarrow{S C}$ & Not likchy & Nut lutely & \\
\hline Yejlow-hesded blackibird & & SC & Mipgation/wirlex & Migrallan'huirtér & \\
\hline
\end{tabular}




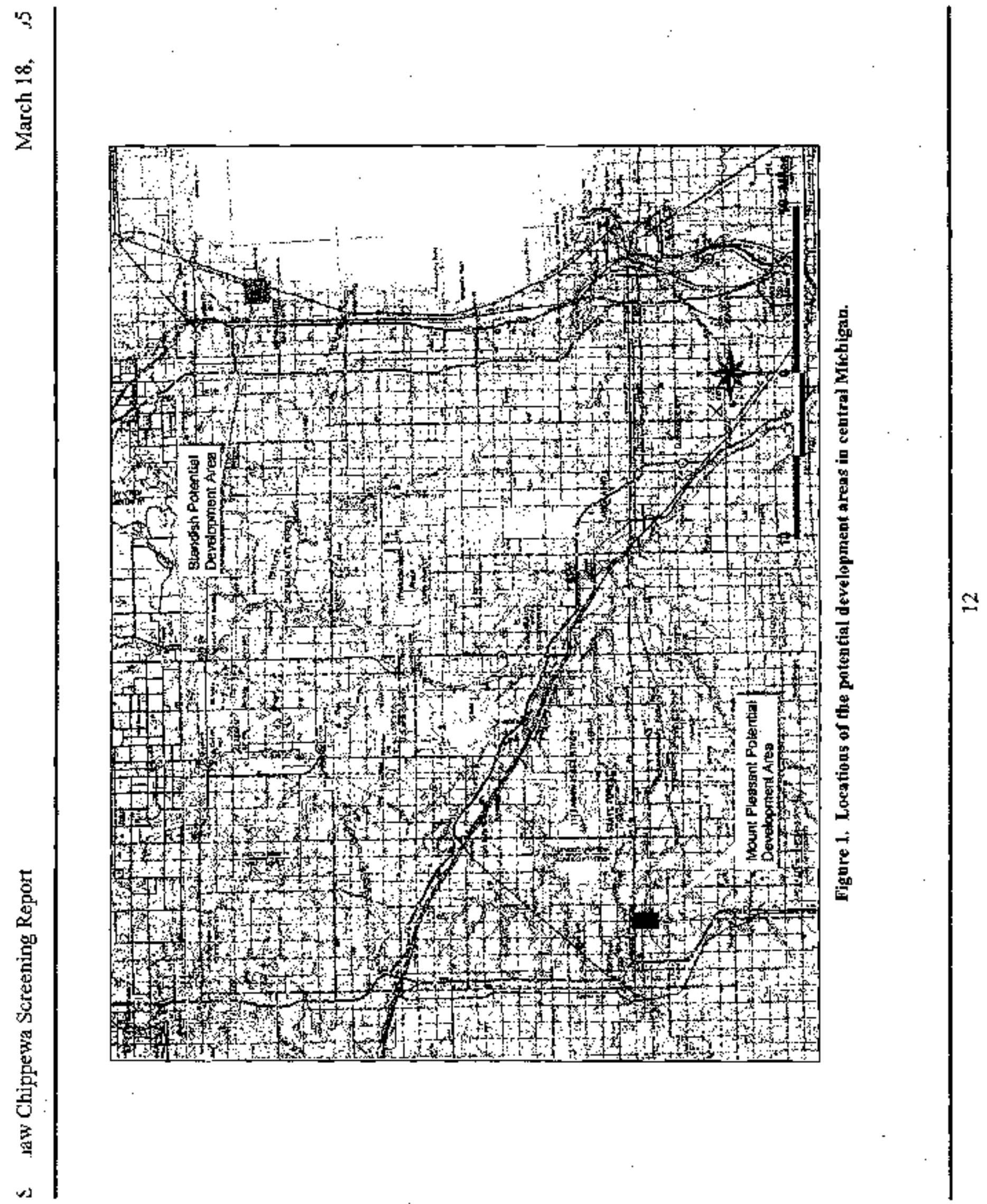




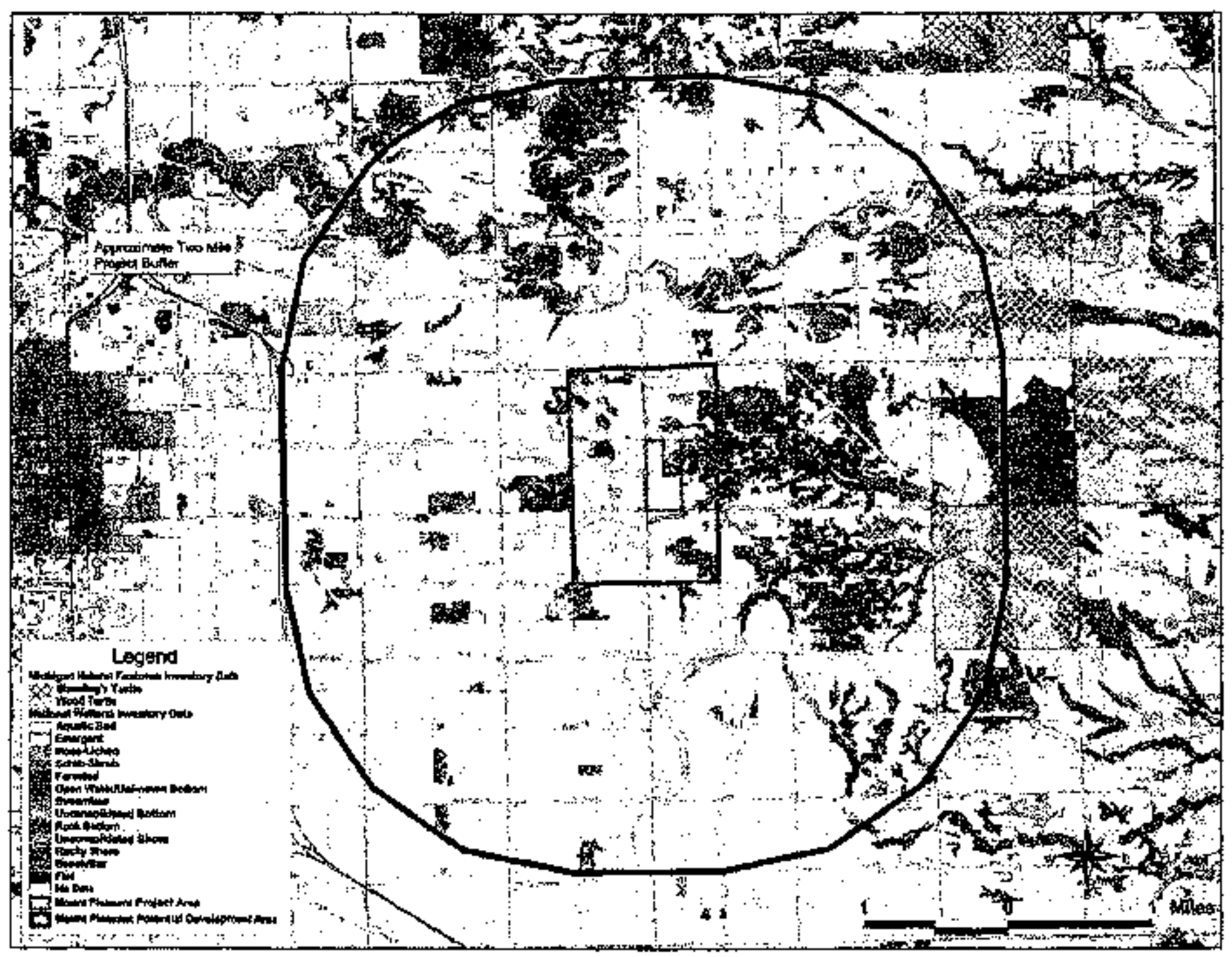

Figare 2. A map of the Mount Pleasanf project area showing wetland and rare species hacations. 


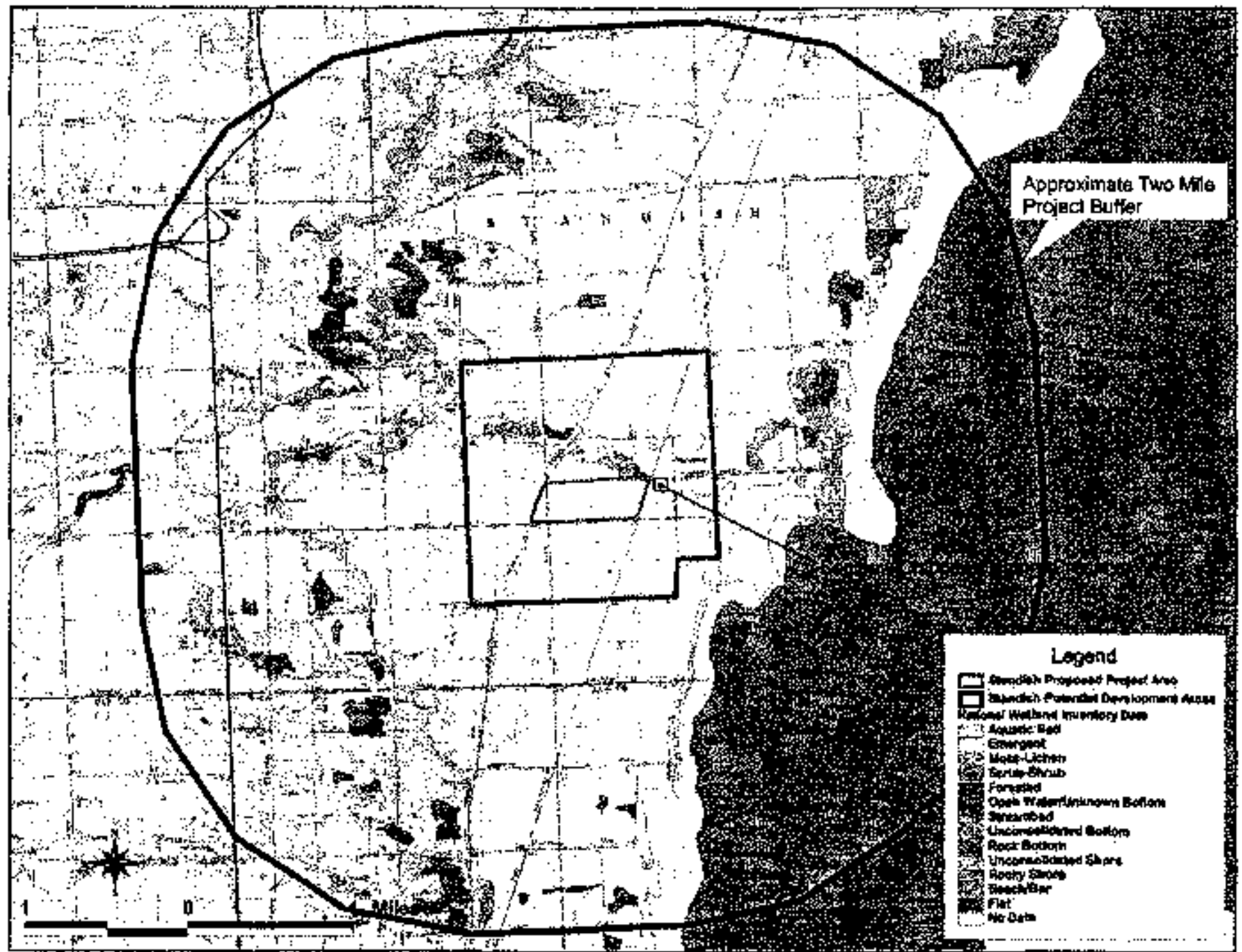

Figure 3. A pap of the Standist project area showlsg wethond locations. The Michigan Natural Features Inventary had no records of rare species bear the project area. 


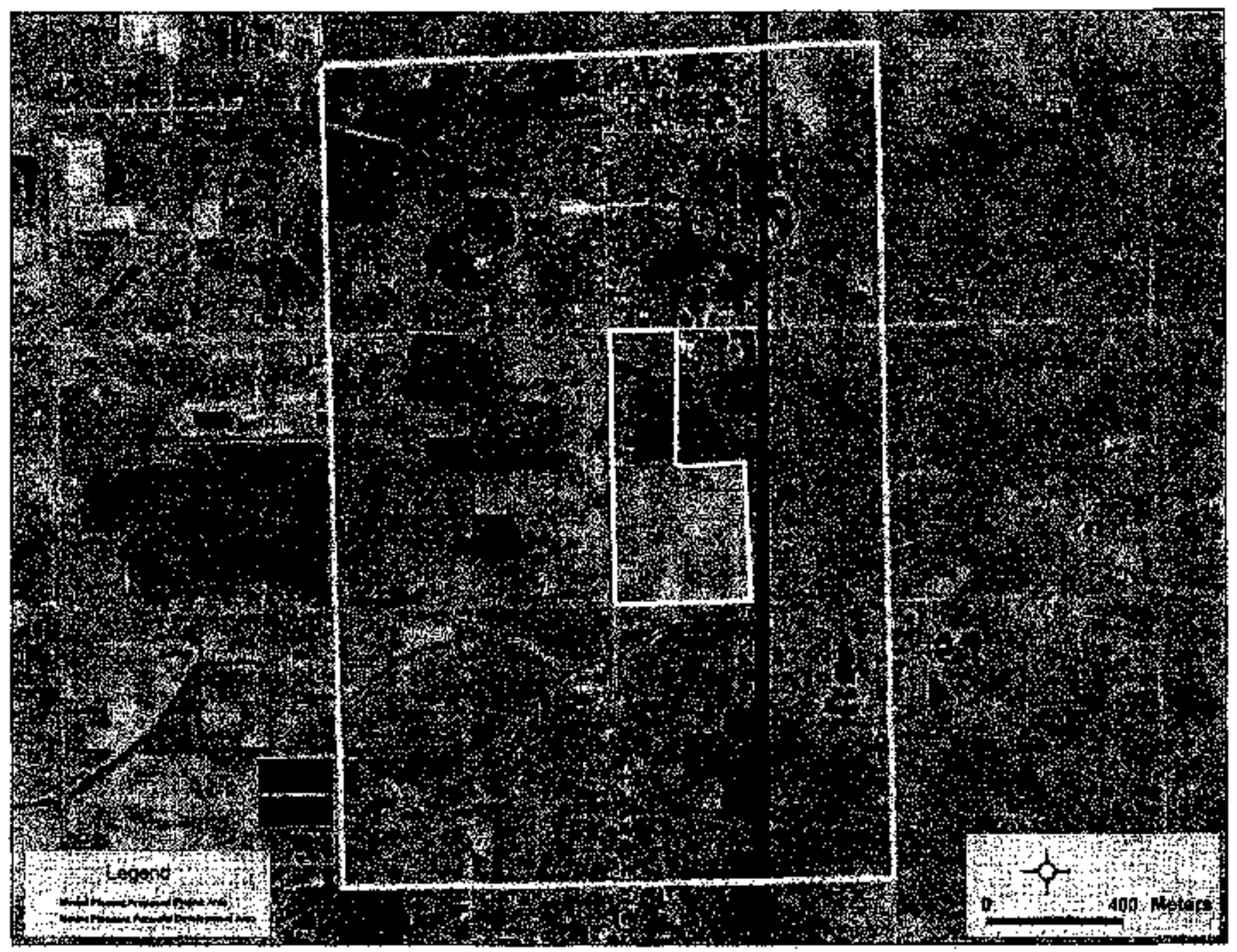

Figure 4. A 1998 merial phipto of the Mount Pleasant proposed project and potential development areas. 


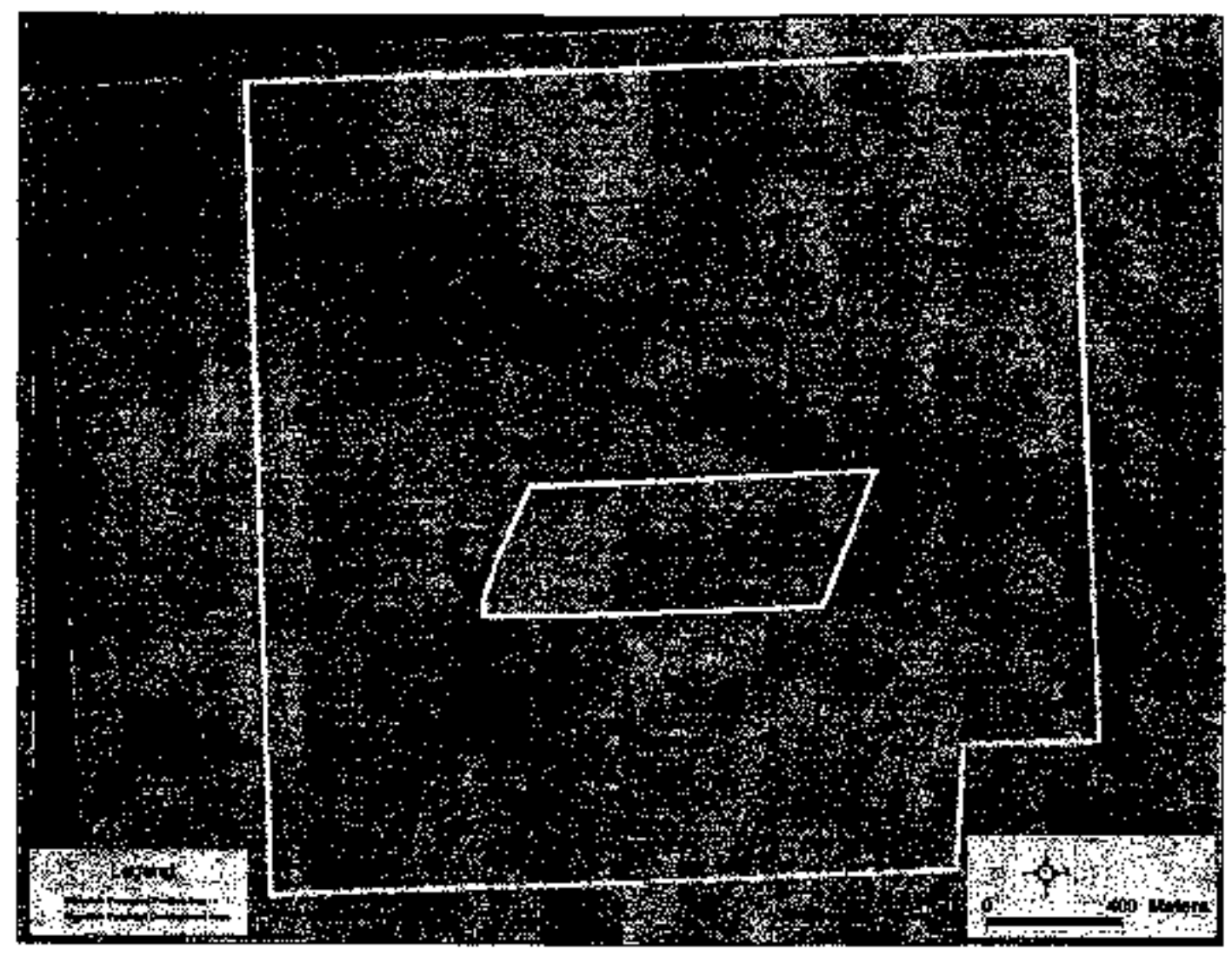

Fienre S. A 1998 aerial photograpb the Siandisb propused project and polebttol development arets. 
Table 5. A summary of the potential for wildtife conflicts in the proposed Mout Pleasant wind development area'. YH = Very High, $\mathbf{H}=$ High, $M=$ Medium, and $\mathbf{L}=$ Low.

\begin{tabular}{|c|c|c|c|c|c|}
\hline Issue & VH & $\mathbf{H}$ & $\mathbf{M}$ & $\mathbf{L}$ & Notes \\
\hline Potential for raptor nest sites & & & 4 & & $\begin{array}{l}\text { The presence of forested } \\
\text { wetlands in the potential } \\
\text { development areta provides } \\
\text { raptor nesting habitat. }\end{array}$ \\
\hline Raptor flight potential & & & & 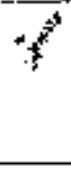 & $\begin{array}{l}\text { The general lack of stark } \\
\text { topography decreases the } \\
\text { potential for concentrated } \\
\text { raptor use. }\end{array}$ \\
\hline Potential for migratory pathway & & & is & & $\begin{array}{l}\text { Buds likely migrate thought } \\
\text { the project areg. }\end{array}$ \\
\hline Potential for raptor prey species & & & [ن & & $\begin{array}{l}\text { Prey densities wete } \\
\text { difficult to assess. }\end{array}$ \\
\hline Potential for protected species to occur & & & 4 & & $\begin{array}{l}\text { Blanding's and Wood } \\
\text { Turtles may be present } \\
\text { within wetland areas. }\end{array}$ \\
\hline Potential for State issues & & & $3 y^{5}$ & & $\begin{array}{l}\text { Some state protected } \\
\text { species likely migrate } \\
\text { through the project area. }\end{array}$ \\
\hline Uniqueness of habitat at wind plant & & & $\dddot{n g}^{4}$ & & $\begin{array}{l}\text { Forested wetlands within } \\
\text { the potential development } \\
\text { area provide more suitable } \\
\text { habitat dhas agriculture. }\end{array}$ \\
\hline Potential for rare plants to occur & & & & $\operatorname{sic}^{4}$ & $\begin{array}{l}\text { Low potential to occur } \\
\text { within agrictltural habitats }\end{array}$ \\
\hline Potential fot use by bats & & it & & & $\begin{array}{l}\text { Presence of forest and } \\
\text { wetlands may increase } \\
\text { local bat use }\end{array}$ \\
\hline Other issues & & & & $x^{6}$ & \\
\hline
\end{tabular}

'Summarized for the project area as a whole but the habitat of the project area varies throughout in its ability to support species of concem. 
Table 6. A sumbary of the potentigl for wildife confilicts in the propesed Standish wind develupment area' ${ }^{\prime}$ VH $=$ Very High, $\mathbf{H}=$ High, $M=$ Mediun, and $\mathbf{L}=$ Low.

\begin{tabular}{|c|c|c|c|c|c|}
\hline Issue & VH & $\mathbf{H}$ & M & $\mathbf{L}$ & Notes \\
\hline Potential for raptor nest sites & & & 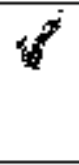 & & $\begin{array}{l}\text { Sopne deciduous woodlots } \\
\text { are preselts within two } \\
\text { miles of the development } \\
\text { area. }\end{array}$ \\
\hline Raptor flight potential & & & 4 & & $\begin{array}{l}\text { The presence of Saginas } \\
\text { Bay may result in increased } \\
\text { migratory raptor use. }\end{array}$ \\
\hline Potenlial for migratory pathway & & $f$ & & & $\begin{array}{l}\text { Areas near the Saginass } \\
\text { Bay Shoreline may be used } \\
\text { as stopover sites by } \\
\text { migrating firds. }\end{array}$ \\
\hline Potential for raptor prey species & & & $\%$ & & $\begin{array}{l}\text { Prey densities were } \\
\text { difficult to assess. }\end{array}$ \\
\hline Potential for protected species to occur & & & 4 & & $\begin{array}{l}\text { Due to proxinity to } \\
\text { Saginaw Bay sonne } \\
\text { potential exists for Bald } \\
\text { Eagle Use }\end{array}$ \\
\hline Potential for State issues & & & sitit & & $\begin{array}{l}\text { Some state profected } \\
\text { species likely migrate } \\
\text { through lie project area. }\end{array}$ \\
\hline Uniqueness of habitat at wind plant & & & "s & & $\begin{array}{l}\text { With the exception of some } \\
\text { wetlands, project area } \\
\text { largety dominated by } \\
\text { agriculure and is not } \\
\text { unique. }\end{array}$ \\
\hline Potential for tare plants to occir & & & & $\sqrt{4}$ & $\begin{array}{l}\text { Low potential to occur } \\
\text { witbin agricultaral habitats. }\end{array}$ \\
\hline Potential for use by bats & & $f^{4}$ & & & $\begin{array}{l}\text { Presence of forest and } \\
\text { wetlands may increase } \\
\text { local bat use }\end{array}$ \\
\hline Other issues & & & & $3^{*}$ & \\
\hline
\end{tabular}

${ }^{1}$ Summarized for the project area as a whole but the habitat of the project area varies throughout in its ability to surport species of concern. 


\section{Literature Cited:}

Avery, M., P. Springer, and J. Cassel. 1976. The effects of a tall tower on noctumal bird migration- a portable ceilometer study. Auk 93:281-291.

Bat Conservation International, Inc (BCI), 2002. Bat Species: U.S. Bats. Bat Conservation International, Inc. Austin, TX. Available at www batconorg

Brewer, R., G. McPeek, and R Adams, Jr. 1991. The Atlas of Breeding Bitds of Michigan. Michigan State University Press, East Lansing, MI. USA

Chartier, A.T. and J. Ztamo. 2004. A Birder's Guide to Michigan. American Binding Association, Colorado Springs.

Dieht, R., R. Larkin, and J. Black. 2003. Radar observations of bird migration over the Great Lakes. Auk 120:278-290.

Erickson, W.P., G.D. Johnson, M.D. Strickland, K.J. Sernka, and R.E. Good. 2001. Avian Collisions with Wind Turbines: A Summary of Existing Studies and Comparisons to Other Sources of Avian Collision Mortality in the United States. Prepared for the National Wind Coordinating Committee. Available at

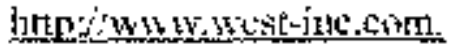

Federal Aviation Administration (FAA). 2000. Obstruction Marking and Lighting. $A C 70 / 7460-1 \mathrm{~K}$.

Gebring, J. 2005. Avian collisions with communication towers: a comparison of tower support systems and tower heights. Annual report.

Kerlinger, P. 1989. Flight strategies of migrating liawks. University of Chicago Press, Chicago, Iltinois, USA.

Kerlinger, P. 1995. How birds migrate. Stackpole, Mechanicsburg, Pennsylvania, USA.

Larkin, R, and B. Frase. 1988. Circular paths of birds flying aear a broadcasting tower in cloud. Journal of Comparative Psychology 102:90-93.

Johnson, G.D. 2003. What is known and not known about bat collision mortality at windplants? In R.L. Carlton, editor. Avian interactions with wind power structures. Proceedings of a workshop in Jackson Hole, Wyoming, USA, October 16-17, 2002. Electric Power Researcli Institute, Palo Alto, California. In Press.

Johnson, G.P., W.P. Erickson, M.D. Strickland, M.F. Shephard and D.F. Shephard, 2000. Avian monitoring studies at the Buffalo Ridge, Minnesota Wind Resource Area: results of a four year study. Westem EcoSystems Technology, Inc. $262 \mathrm{pp}$. 
Johnson, G.D., D.P. Young, Jr., W.P Erickson, C.E. Derby, M.D. Strickland and R.E. Good. 2000. Wildlife Monitoring Studies, SeaWest Windpower Project, Carbon County, Wyoming, 1995-1999. Prepared for SeaWest Energy Corporation, San Dieago, CA, and Bureau of Land Management, Rawlins.

Michigan Natural Features Inventory (MNFT) 1999. Micligan's Special Animals: Endangered, Threatened, Special Concern, and Probably Extirpated. Available at

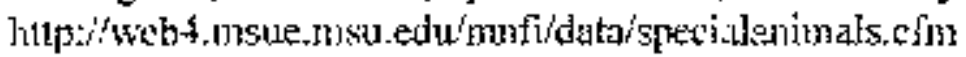

National Wind Coordinating Committee (NWCC) 2004. Wind Turbine Interactions with Birds and Bats: A summary of Research Results and Remaining Questions. Fact Sheet, Second Edition. 


\section{Appendix A - USFWS and Michigan DNR Correspondence}




\title{
United States Department of the Interior
}

\author{
FISH AND WTLDLIFL SERVICE \\ Hisl lansing lïcld (ritice \{L:S) \\ 3451 Conjidge Roat, Sujte 101

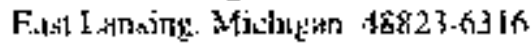

IN KRTLIS RTLLRTS.

Felruary 24. zons

Rhent Gont

Westurn Ecosystens Technology, Inte.

200 ;

('husculat. Wyoning \$2001

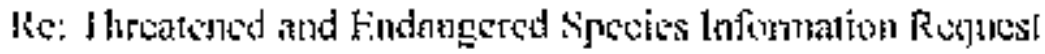

Dear Mr. tiond:

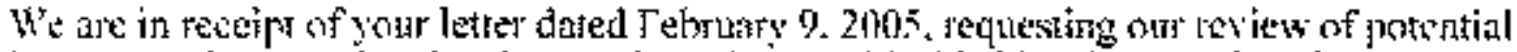
iruptecls th threitlented and endingered species or critieal habilet lor two sites that may bu

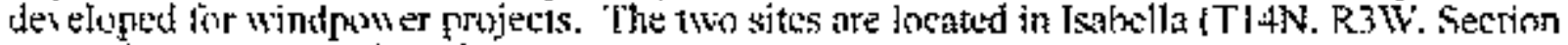

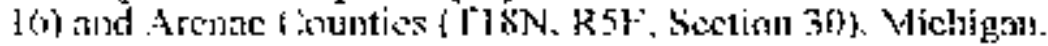

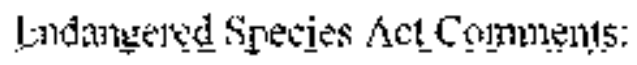

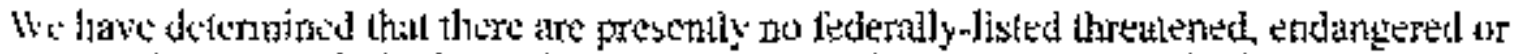
propoved species within fle projict aled. Ihis prectudes the need for futher action on this

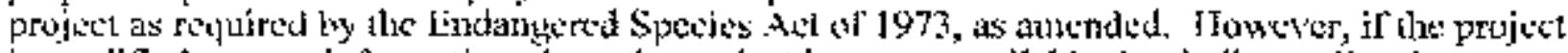
is mocificd or new infumation about the project becones axailable that indicares listed or

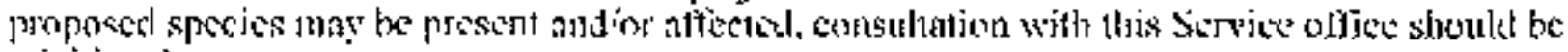
rejialialutel.

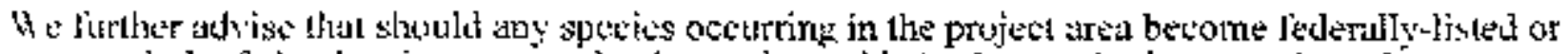
proposed. lhe fedenal action ageney for the work would also be required to reesaluate is

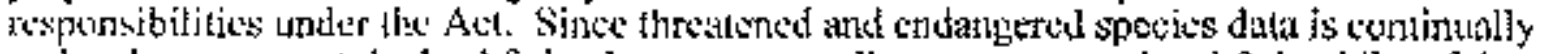
updated. we suggost the lead federal agercy anjual y request an updaded federal hist of the

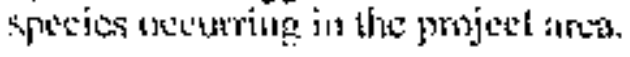

We appreciate the opportumity to provide ljese comments and look forward io any futur:

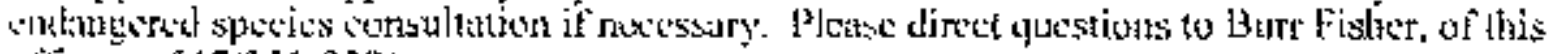
alies, at $517,351-8286$.

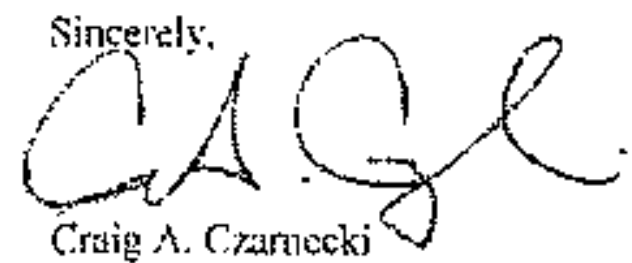

Fickl Suparvisur

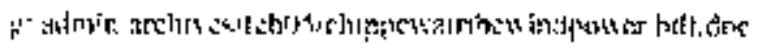


$\therefore$ ㄴ. anctsis
DEPARTMENT OF NATURAL RESOURCES

isin.

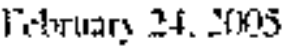

AEEECOA A. HLUTFHRES

Dins $e^{2} r^{2}$

\section{Atr. Rlach l: Good}

Witurn Ecrsy'stetus Fechnolong. Ine.

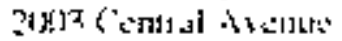

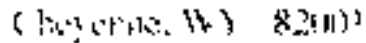

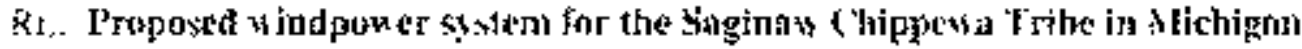

Dreac $\mathrm{N}_{1}$ (

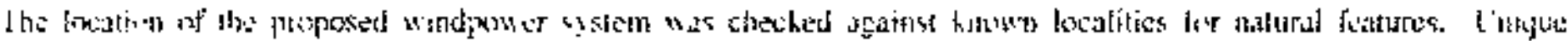

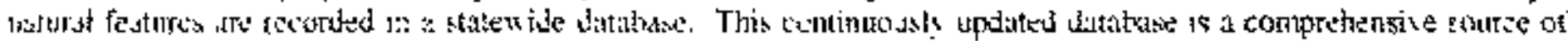

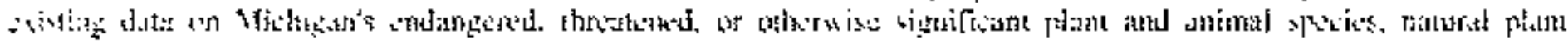

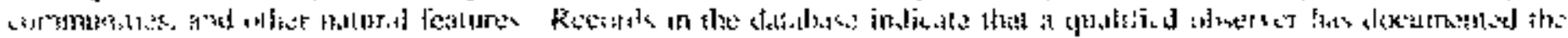

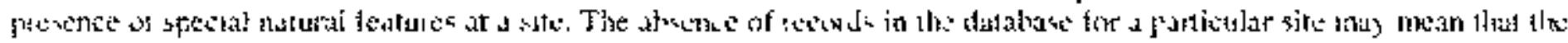

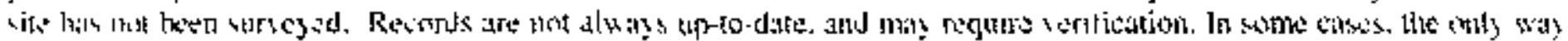

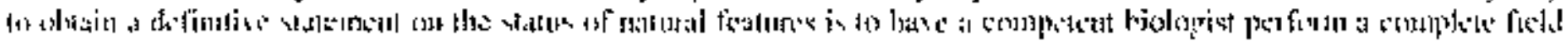
(अT:5)

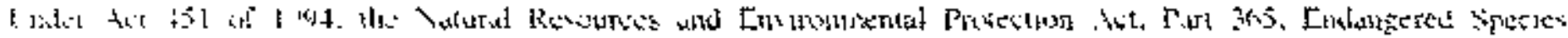

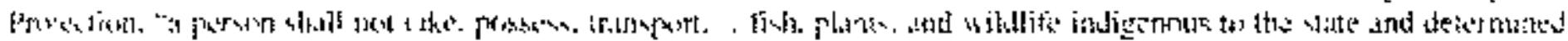

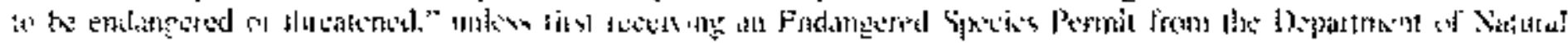

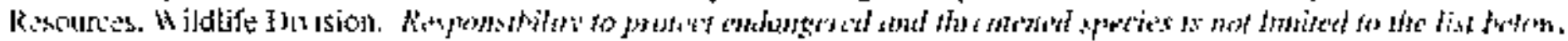

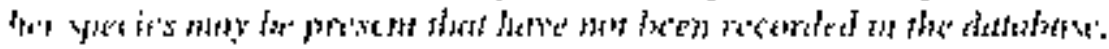

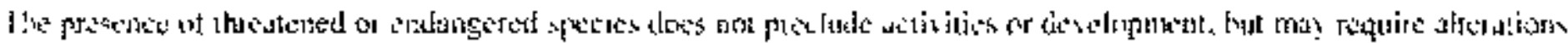

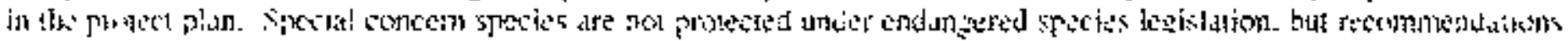
: : 1915 a

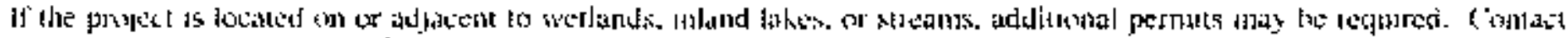

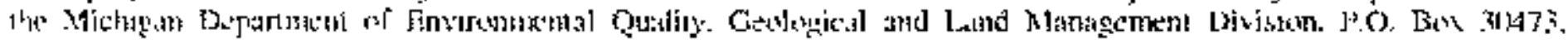

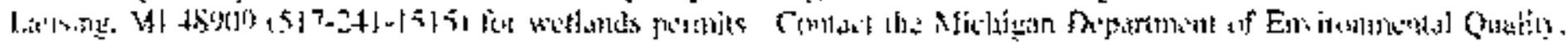

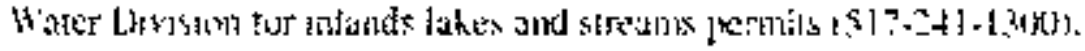

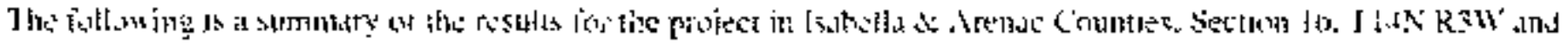

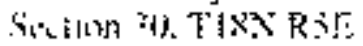

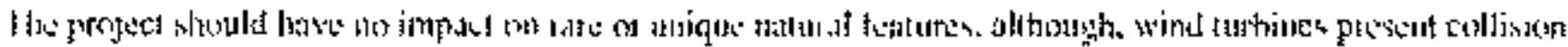

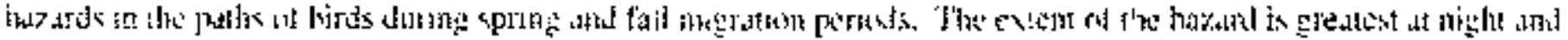

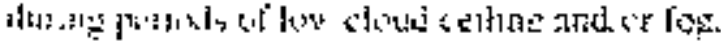

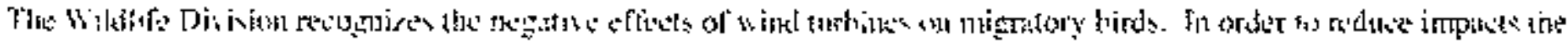

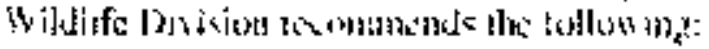

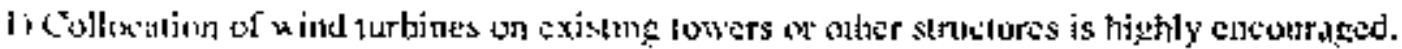




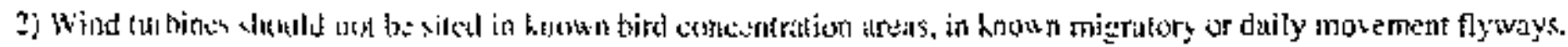

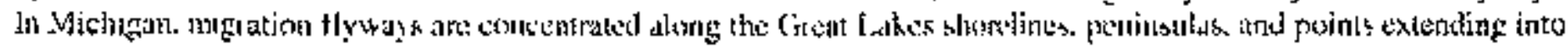

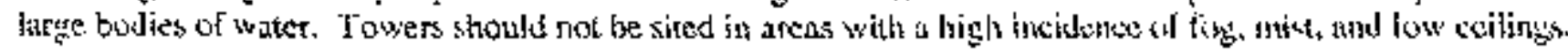

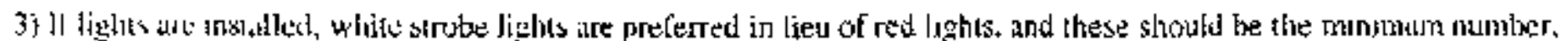

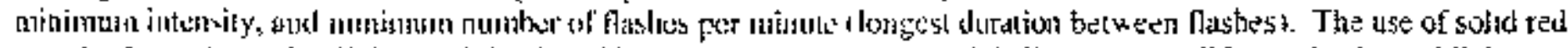

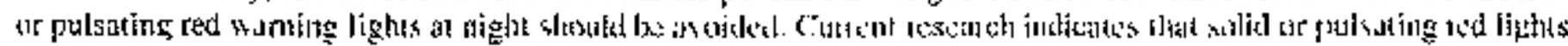

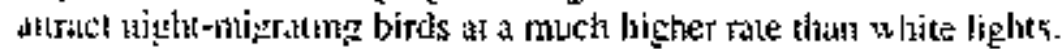

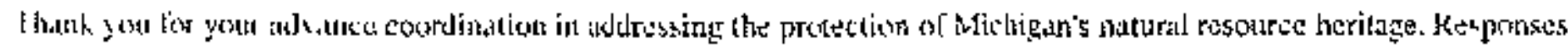

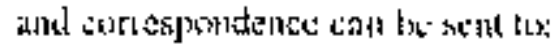

Michigin Depatment of Nagural Resources

Wildlilic Divinion - Natural J Keribage Prongam

P() Bis

Latsing. V] 18 the

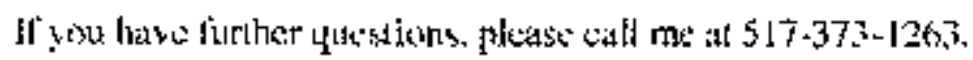

Sincerdy.

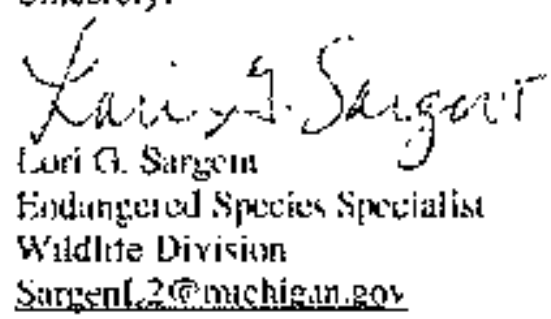




\section{APPENDIX B}

\section{POTENTIAL IMPACT INDEX CHECKLISTS}




\begin{tabular}{|c|c|c|c|c|c|c|c|}
\hline \multirow{2}{*}{\multicolumn{4}{|c|}{ Physical Attribute }} & \multicolumn{4}{|c|}{ Site } \\
\hline & & & & $\begin{array}{l}\text { Mournt } \\
\text { Pletisant }\end{array}$ & Standish & Tawas Point & $\begin{array}{l}\text { Nayanquing } \\
\text { Point }\end{array}$ \\
\hline \multirow{15}{*}{ Topogaphy } & \multirow{9}{*}{ 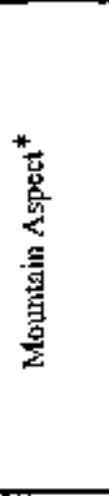 } & \multirow{4}{*}{ Sije } & $w$ & & & & \\
\hline & & & $E$ & & & & \\
\hline & & & $\mathrm{N}$ & & & & \\
\hline & & & S & & & & \\
\hline & & \multicolumn{2}{|c|}{ Top } & & & & \\
\hline & & \multirow{4}{*}{ Foothill } & $w$ & & & & \\
\hline & & & E & & & & \\
\hline & & & $N$ & & & & \\
\hline & & & $s$ & & & & \\
\hline & \multicolumn{3}{|l|}{ Valley* } & & & & \\
\hline & \multicolumn{3}{|l|}{ Piss* } & & & & \\
\hline & \multicolumn{3}{|l|}{ Gap* } & & & & \\
\hline & \multicolumn{3}{|l|}{ Ridge* } & & & & \\
\hline & \multicolumn{3}{|l|}{ Biluff* } & & & & \\
\hline & \multicolumn{3}{|l|}{ Spit* } & & & $\mathrm{x}$ & $\mathrm{x}$ \\
\hline \multirow{5}{*}{$\begin{array}{c}\text { Wind* } \\
\text { Direction }\end{array}$} & \multicolumn{3}{|l|}{$s$} & & & & \\
\hline & \multicolumn{3}{|l|}{$\mathrm{N}$} & & & & \\
\hline & \multicolumn{3}{|l|}{ E } & & & & \\
\hline & \multicolumn{3}{|l|}{$w$} & $x$ & $\mathrm{x}$ & $x$ & $\mathrm{x}$ \\
\hline & \multicolumn{3}{|c|}{ UIdrafts* } & & & & \\
\hline \multirow{5}{*}{$\begin{array}{l}\text { Mistallory* } \\
\text { Corritor } \\
\text { Potential }\end{array}$} & \multicolumn{3}{|c|}{ Latitudinat $(N-S)$} & & $x$ & $\mathrm{x}$ & $\mathrm{x}$ \\
\hline & \multicolumn{3}{|c|}{ Longitudinal $(\mathrm{E} \cdot \ldots \mathrm{W})$} & & & & \\
\hline & \multicolumn{3}{|c|}{ Wide Approaches (>30 km)" } & & & & \\
\hline & \multirow{2}{*}{$\begin{array}{l}\text { Punnei } \\
\text { Effect"* }\end{array}$} & \multicolumn{2}{|l|}{ Horizontal } & & $x$ & $x$ & $x$ \\
\hline & & \multicolumn{2}{|l|}{ Vertical } & & & & \\
\hline & $<640$ & & & $\mathrm{x}$ & $\mathrm{x}$ & $x$ & $\mathrm{x}$ \\
\hline Site Size & $>640<1$ & & & & & & \\
\hline Configuration* & $>1000$ & 1500 & & & & & \\
\hline & Tutbine & Rows not Pas: & to Migration & & & & \\
\hline & Transm & sion & & $\mathrm{x}$ & $x$ & $x$ & $\mathrm{x}$ \\
\hline & Roads & & & $\mathrm{x}$ & $\underline{x}$ & $x$ & $x$ \\
\hline [iffrastructurt & Bujidin & $s^{*}$ & Storage & $x$ & $x$ & $\underline{x}$ & $x$ \\
\hline To Build & & & Mainsezance & $\mathrm{x}$ & $x$ & $\mathrm{x}$ & $\mathrm{x}$ \\
\hline & & & Daily Actiyity & $\mathrm{x}$ & $\mathrm{x}$ & $x$ & $x$ \\
\hline & Substati & & & $x$ & $x$ & $\mathrm{X}$ & $\mathbf{X}$ \\
\hline Irereased Activi & & & & $\mathrm{x}$ & $x$ & $\mathrm{x}$ & $\mathrm{x}$ \\
\hline & & & Torals & 9 & 11 & 12 & 12 \\
\hline
\end{tabular}


PHYSICAL ATTRIBUTE CRITERIA - 36 categories, max $•=36$.

Topography - Terzain characteristic within the ecological influence of the proposed wind farm, generally, but not restricted to $\pm 8 \mathrm{~km}$.

Mountain Aspect - Aspect of topogzaphy for site of proposed development. Multiple categories may be checked.

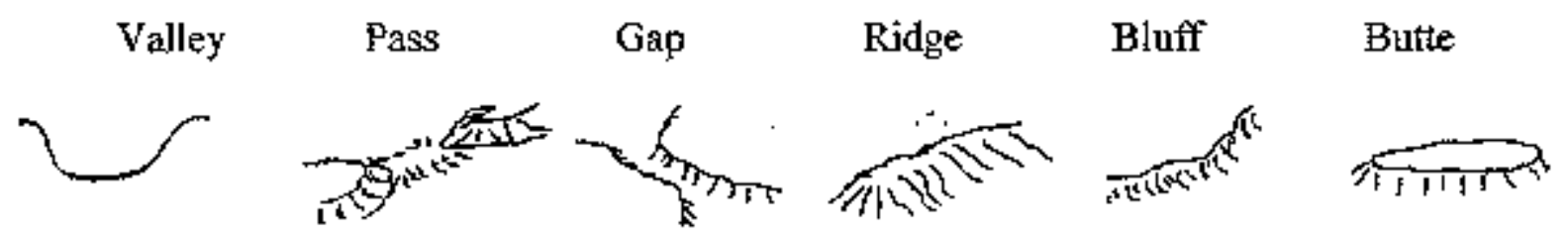

Wind Direction - Compass direction from which prevailing winds approach. Multiple categories may be checked.

Updrafts - Do updrafts'upsiope winds prevail?

Migratory Corridor Potential - Subjective estimate of area to be a potential aviau/bat migratory conidor based strictly on topographical characteristics. Multiple categories may be checked.

Wide ( $>30 \mathrm{kn})$ - Terrait chazacteristics of approaches to site from each migratory direction, $i_{1} e_{+}$a

large plain, river comidor, long valicy. The larger the area that migrant birds/bats are drawn from, the more may be at tisk

Fonnel Effect - Is the site in or near an area where migrant birds/bats may be funneled (concentrated) into a smaller area, etther altitudinally, laterally, or both?

Site Size \& Configuration - Size is estimated as if a minimum convex polygon (MCP) were drawn around peripheral turbines.

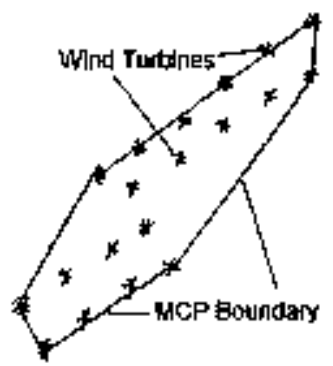

Successive boxes are checked to convey relationship of larger size = inereased inpact to birds/bats, e.g., a 700 acre site will have 2 categories checked while a 1200 acre site will have al] 3 categories checked.

Configuration of turbine rows is usually perpendicular to prevailing wind direction. Rows aligesed perpendiculat or oblique to roule of migtation intuitively preserts more risk to biros than rows aligned parallel to movement.

Buildings - Building are categorized by relative size and visitation frequency, i.e., structures that are visited daily are ușually larger and present more impact than those that are not. If a "Daily Activity" building is required, all Building categories are checked. If a maintenarce structure is required, Storage is also checked.

Increased Activity - Will any type of humatz activity increase? Sites in urbannsuburban or otherwise developed areas (oil, gas, mines) will have less impact on vertebrate wildifie than those in remote or undeveloped areas. 
Avian Species of Special Concem Checklist (Complete prior to SPECEES OCCURRENCE \& STATUS CHECKLIST)

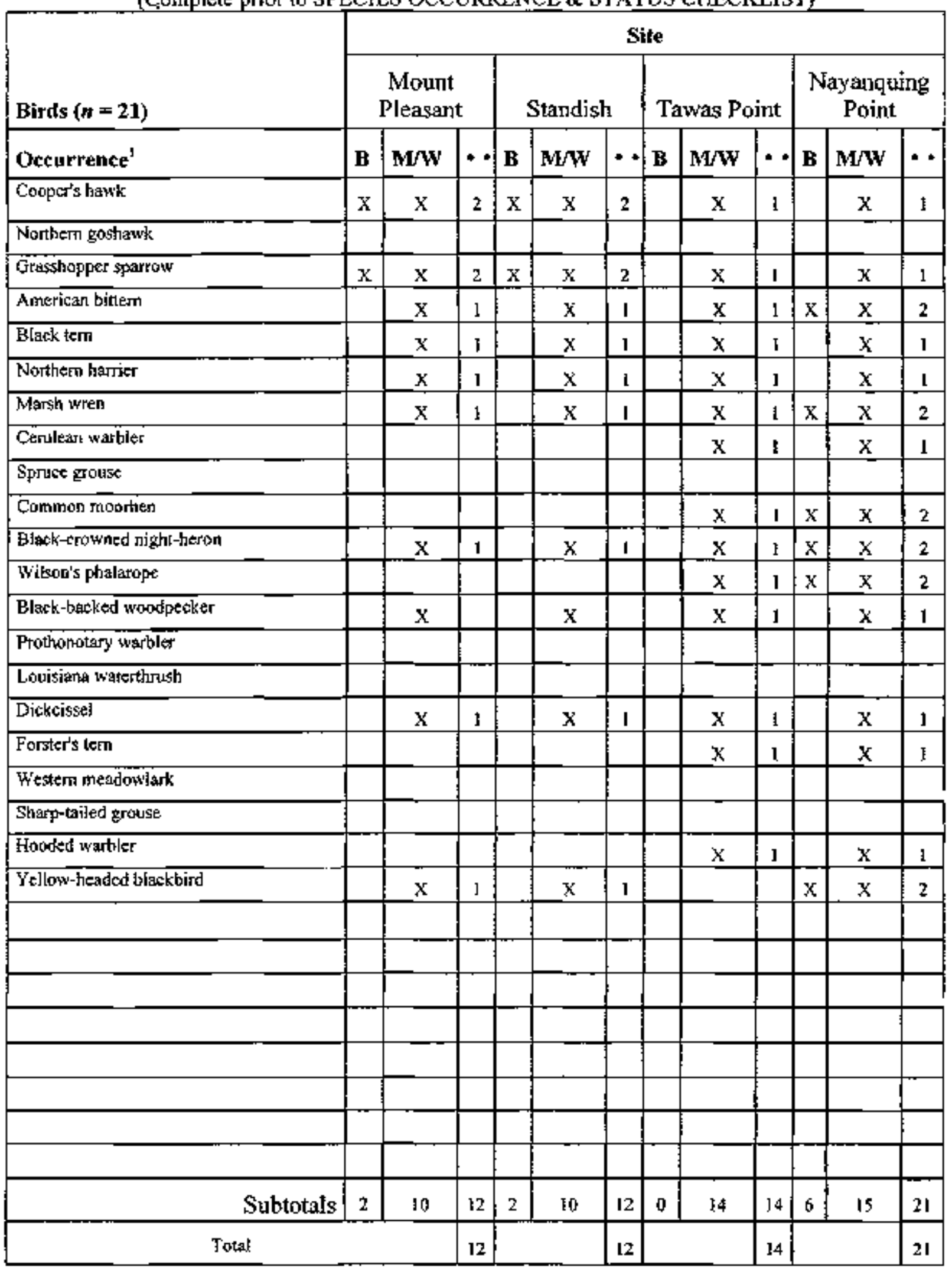


Avian Species of Special Concern Checkist (2l species, max $\cdot=42$ )

Column totals of this list are added to appropriate celis in the SPECIES OCCURRENCE \& STATUS CHECKLIST. Appropriate ayjan field gaides and species accounts should be consulted for confurmation of species distribution and habitat associations.

In addition to species lists (rows), season of occutrence is also indicated (columns). " $B$ " indicates breeding or summer occurrence and " $\mathrm{M} / \mathrm{W}$ " indicates presence during migration or as wintering species. The USFWS guidelines for windpower dewelopment suggests that if occurence within or in the vicinity ( $+7 \mathrm{~km}$ ) of a proposed site is confirmed or suspected, an "X" is entered. However, dwe to sharp differences in habitat and topography withit $7 \mathrm{~km}$ of the proposed project, and $X$ was only entered if it was likely the species would occur or fly througl the project area based of topograply and habitat features.

NOTE: These species were selected because they are listed as species of concern by the Michigan Department of Natural Resources Determinations of occurrence were based on the geographical location of the project area, lubitat, Chartier and Ziarno 2004, Breeding Bird Survey Data, Christmas Bird Count data, and the 1991 edition of the Atlas of Breeding Birds of Michigan 
Bat Species Of Special Concern Checklist

(Complete prior to SPECIES OCCURRENCE \& STATUS CHECKLIST)

\begin{tabular}{|c|c|c|c|c|c|c|c|c|c|c|c|c|}
\hline \multirow{3}{*}{$\begin{array}{l}\text { Bats }(n=2) \\
\text { Oecurrence }\end{array}$} & \multicolumn{12}{|c|}{ Site } \\
\hline & \multicolumn{3}{|c|}{$\begin{array}{l}\text { Mount } \\
\text { Pleasant }\end{array}$} & \multicolumn{3}{|c|}{ Standish } & \multicolumn{3}{|c|}{ Tawas Point } & \multicolumn{3}{|c|}{$\begin{array}{l}\text { Nayanquing } \\
\text { Point }\end{array}$} \\
\hline & $\mathbf{B}$ & $M / w$ & * & B & Mw & $\cdots$ & B & M/W & $\cdot \cdot$ & B & M/W & $\cdots$ \\
\hline Indiana Bat & 0 & 0 & 0 & 0 & 0 & 0 & 0 & 0 & 0 & 0 & 0 & 0 \\
\hline Eastern Pinjstrelle & 0 & 0 & 0 & 0 & 0 & 0 & 0 & 0 & 0 & 0 & a & 0 \\
\hline & & & & & & & & & & & & \\
\hline & & & & & & & & & & & & \\
\hline & & & & & & & & & & & & \\
\hline Subtotals & 0 & 0 & 0 & 0 & 0 & 0 & 0 & 0 & 0 & 0 & 0 & 0 \\
\hline Total & & & 0 & & & $0:$ & & & 0 & & & 0 \\
\hline
\end{tabular}


Bat Species Of Special Concem Checklist (2 species, max * $=4)$.

Column fotals of this tist are added to apptopriate cells in the SPECIES OCCURRENCE \& STATUS CHECKLIST. Appropriate bat field gitdes and references should be consulted for confimation of species distribution and habitat associations.

In addition to species lists (rows), season of occurrence is also indicated (columns). " $\mathrm{B}$ " indicates breeding or summer occurence and "M/W" indicates presence during migration or as wintering species. If occurrence withis or in the vicitity $(\cdot 7 \mathrm{~km})$ of a proposed site is confirmed or suspected, an "X" is entered.

NOTE: These species were selected because they are listed on the Michigan Endangered Species List or are a specles of concern. Occurrence in each area was based on bat distribution data available from Bat Conservation International (2003). 
SPECIES OCCURRENCE \& STATUS CHECKLIST

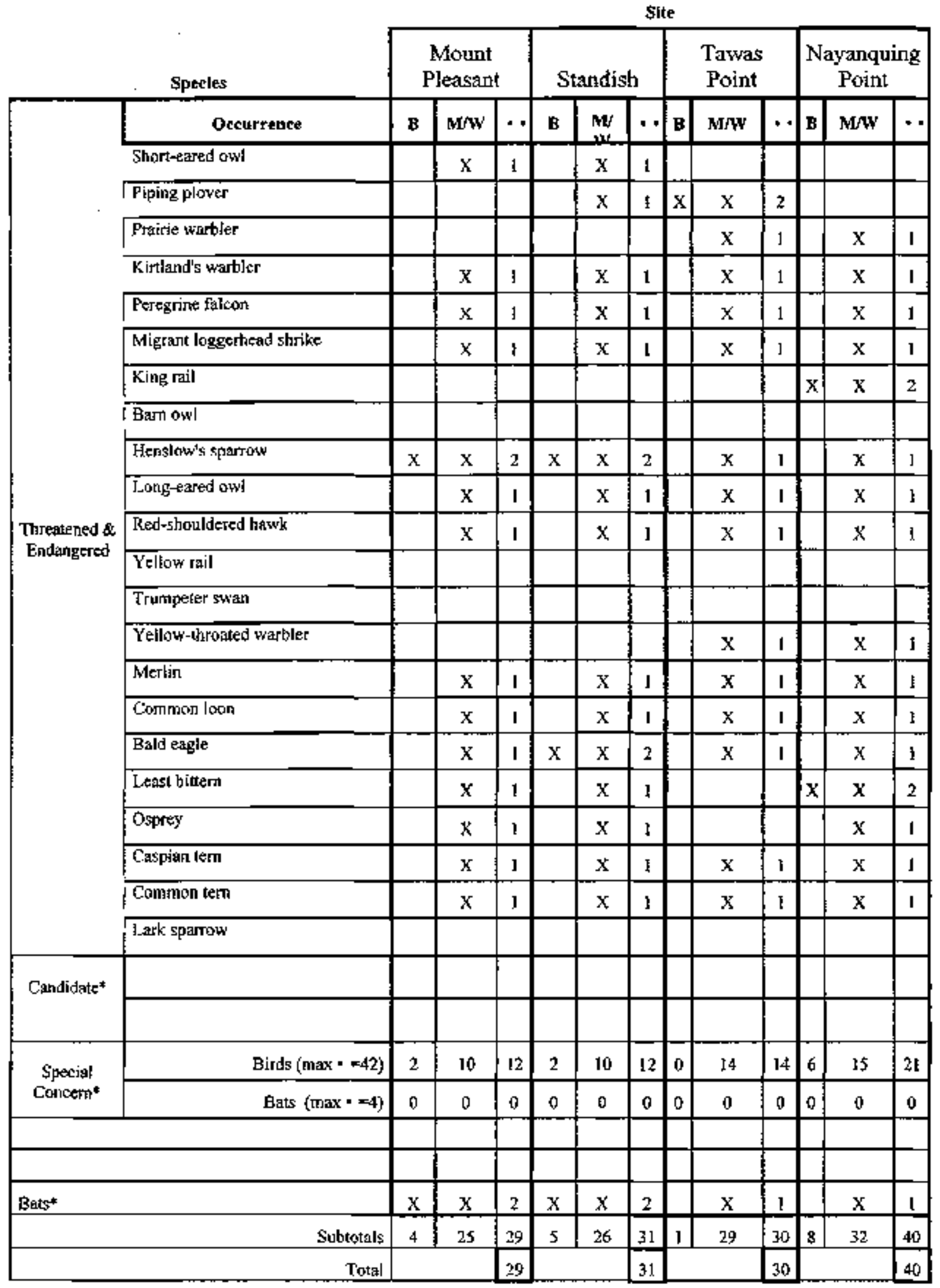




\section{SPECIES OCCURRENCE \& STATUS CHECKLIST ( 46 categories, $\max *-\mathbf{9 2 )}$}

Checklist totals for each column in "Avian Species of Special Concern List" and "Bat Species of Special Concem List are inserted in this checklist.

Threatened \& Endangered Species - Species include in the Federal List of Endangered and Threatened Species. Only bird species were included. Bat species are included within the Bat section. Other groups such as mollusks, anphibians, reptiles aud insects may be present, however, they are assumed to not be impacted if only agricultural habitats are targeted for development

Candidate Species - Species being investigated for inclusion in the Federal List of Endangered and Threatened Species.

Species of Special Concern - Birds of concem list obtained fron the Michigan Natural Features Inventory website.

Bats (otber than bat Species of Special Concern) are included đue to generally unknown impacts of wind farms on individual and populations. 
ECOLOGICAL ATTRACTIVENESS CHECKLIST

\begin{tabular}{|c|c|c|c|c|c|c|}
\hline \multirow{2}{*}{\multicolumn{3}{|c|}{ Ecological Attractor }} & \multicolumn{4}{|c|}{ Site } \\
\hline & & & \multirow[t]{2}{*}{$\begin{array}{l}\text { Mount } \\
\text { Pleassant }\end{array}$} & \multirow[t]{2}{*}{ Standish } & \multirow{2}{*}{$\begin{array}{c}\begin{array}{c}\text { Tawas } \\
\text { Point }\end{array} \\
\mathrm{x} \\
\end{array}$} & \multirow{2}{*}{$\begin{array}{c}\begin{array}{c}\text { Nayanguing } \\
\text { Point }\end{array} \\
x\end{array}$} \\
\hline \multirow{5}{*}{$\begin{array}{c}\text { Migration } \\
\text { Ronte* }\end{array}$} & & Local & & & & \\
\hline & \multirow{4}{*}{ Continental* } & $\mathrm{N}$ & $\mathrm{x}$ & $x$ & $\mathrm{x}$ & $\mathrm{x}$ \\
\hline & & $s$ & $\mathrm{x}$ & $\mathrm{x}$ & $\mathrm{x}$ & $\mathrm{x}$ \\
\hline & & $\mathrm{E}$ & & & & \\
\hline & & $\mathrm{W}$ & & & & \\
\hline \multirow{9}{*}{$\begin{array}{l}\text { Ecological } \\
\text { Magnets* }\end{array}$} & \multicolumn{2}{|c|}{ Lotic System } & & & & \\
\hline & \multicolumn{2}{|c|}{ Lentic System } & & $\mathrm{x}$ & $x$ & $x$ \\
\hline & \multicolumn{2}{|r|}{ Wetlands } & $x$ & $\mathrm{x}$ & $\mathrm{x}$ & $\mathrm{x}$ \\
\hline & \multicolumn{2}{|c|}{ Native Grassiand } & & & & \\
\hline & \multicolumn{2}{|r|}{ Forest } & $x$ & $x$ & & \\
\hline & \multicolumn{2}{|c|}{ Food Concentrated } & & & $\mathrm{x}$ & $\mathrm{x}$ \\
\hline & \multicolumn{2}{|c|}{ Energetic Foraging } & & & $x$ & $\mathrm{x}$ \\
\hline & \multirow{2}{*}{$\begin{array}{c}\text { Vegetation } \\
\text { Fabitat }\end{array}$} & Unique & & & $\mathrm{x}$ & $x$ \\
\hline & & Diverse & & & $\mathrm{x}$ & $\mathrm{x}$ \\
\hline \multicolumn{3}{|c|}{ Significant Ecological Event" } & & & $x$ & $\mathrm{x}$ \\
\hline \multicolumn{3}{|c|}{ Site of Special Conservation Status* } & & & $x$ & $\mathrm{x}$ \\
\hline \multicolumn{3}{|r|}{ Total } & 4 & 5 & 11 & 11 \\
\hline
\end{tabular}


ECOLOGICAL ATTRACTTVENESS CRITERLA - 16 categoties, max $=17$.

Migration Route - Indicates predominate direction of movement of seasonal migrations. Multiple categories may be checked.

Local - Some avian populations move onty altitudinally \& direction may be East-West (sage grouse, owis, bałd eagles).

Continental - Sonje migratory corridors experience mass movements in only one season/direction anntially (e g., Bridger Mountains autumn eagle migration).

Ecologicat Magnets - Special, unique, umusual, or syper ordinary habitats or conditions within the vicinity of the site that may attract vertebrate wildife. Lotic systems include small perennial or seasonal creeks to major tivers. Lentic systems inchude stock ponds to lakes. Multiple categories may be checked.

Vegetation/Habitat - Unique or exceptionally diverse vegetation or habitat in the vicinity may indicate exceptional diversity and abundance of avian species or bats.

Significant Ecological Event - Special, unique, unusual, or super ordinary events that occur or are suspected to occur in the vicinity of the site, e g., up to one third of the Continental population of Trampeter Swans visit Ennis Lake, <4 km from a proposed Wind Resource Area; the Continental migration of shorebirds passes over (many stop) @ Benton Lake National Wildlife Refuge) and up to 2000 golden eagles pass over the Bridger Mountains in autumn. If unknown but suspected a "in" is entered. Specifics regarding the cell are then addressed in the appropriate box of the SITE SPECIFIC COMMENTS sheet to focus follow-up investigation and assist in definition of study objectives.

Site of Special Conservation Status - Any existing or proposed covenants, conservation easements, or other land development limitations intended to conserve, protect, or enhance wildlife or habitat. This criterion is weighted ( 2 entered if true) because of previous financial ot other investment in ecological values. Specifics regarding the easement are then addressed in the appropriate box of the SITE SPECIFIC COMMENTS sheet to focus follow-up atiention. 


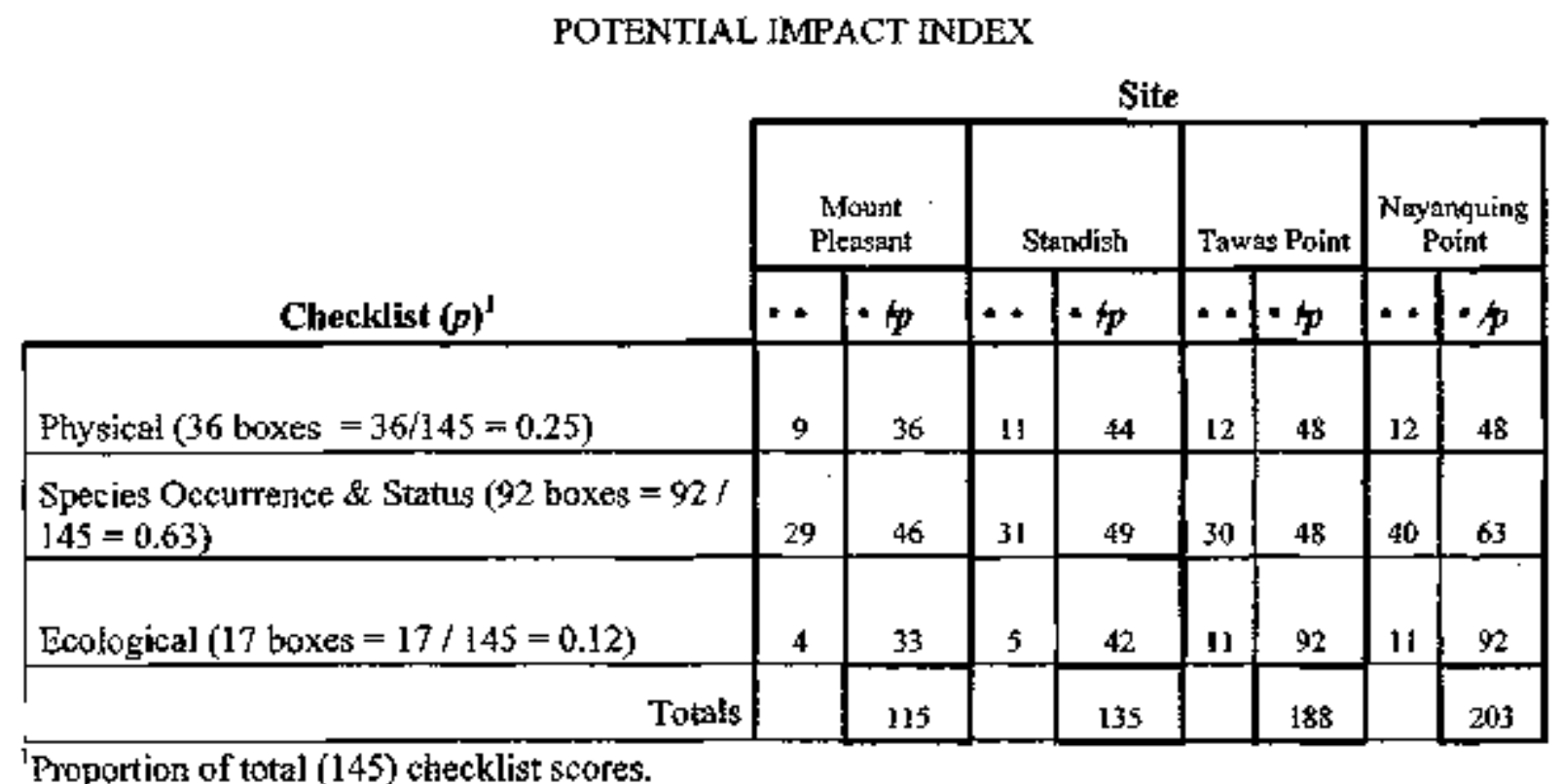


Appendix K. Preliminary Cultural Assessment 


\title{
PRELIMINARY CULTURAL ASSESSMENT \\ FOR PROPOSED \\ WIND TURBINE SITES \\ FOR THE \\ SAGINAW CHIPPEWA INDIAN TRIBE \\ OF MICHIGAN
}

\author{
PREPARED BY \\ Anita Heard, Research Center Coordinator \\ and \\ Robin L. Spencer, Research Center Specialist
}




\section{INTRODUCTION:}

\section{Background}

The Saginaw Chippewa Indian Tribe (Tribe) is a federally recognized sovereign nation, whose tribal lands are located in both Isabella and Arenac Counties in the State of Michigan. The Isabelia Reservation in Isabella County has 138,240 acres within the boundaries, of which 2000 acres are held by the Tribe in fee simple and trust status. Another approximately 700 acres are designated as altotted lands. The Isabella Reservation in Arenac County bas 70 acres of trust land. The Tribe also owns approximately 430 acres of land in fee simple as of reservation lands.

The Tribe was awarded a United States Department of Energy (DOE) grant DE-FC36-04G014252 for funding a feasibility study designed to investigate the possibility of developing a wind power facility on two sites on or near the Isabella reservations. Part of the deliverable task was to investigate any potential cultural impacts that may affect a wind energy development sites. Disgen contracted with the Zibiwind Center of Anishinabe Cultural \& Lifeways to conduct this study. The cuitural study includes the following:

\section{Scope of Work for proposed Wind Turbine Sites}

- Historical Review of Past use of the șite

- Records search of existitg archeological/culfural items on proposed site.

- Interviews with knowledgeable tribsl elders regarding cultural significant about the past and present activities undertaken within the proposed site.

- Report of findings and recommendation from the Tribal Historic Preservation office, if any.

Two sites were chosen for proposed wind turbines for the Saginaw Chippewa Indian Tribe of Miclyigan, one within the reservation boundaries in Mt. Pleasant, Michigan that consists of 39 acres and the other within the reservation boundaries on the Saganing reservation, in Standish, Michigar cousisting of 97 acres. (See B-1 \& B-2)

\section{PROPOSED SITES AREAS:}

The first proposed site location is in Mt. Pleasant, Isabella County, Union Township, section 16 at the cormer sotsth of Remus Rd. and west of Shepard Rd, with the exception of the 1 acre right at the corner. The GIS coordinates for the site are (Longitude $43^{*} 35.951^{\prime} \mathrm{N}$ and Latitude $84^{*}$ $41.193^{\circ} \mathrm{W}$ ). This site consists of 39 acres of tribally owned property known as the Benzinger II site. (Maps included, B-3, B-4)

Legal description: part of the SW $1 / 4$ of Section 16, T14N-R3W, Chippewa Township, Isabella County, Michtgan, described as beginning as a point on the West Section line, which is $\mathrm{N} 00^{*}$.

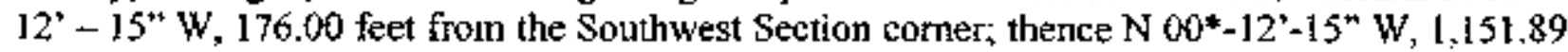
feet; thence N 89*-48'-45" E, 1,312.94 feet along the South - E \& W 1/8 line as occipied; thence $S 00^{*}-15^{\prime}-05^{\prime \prime} \mathrm{E}, 1,329.99$ feet along the West $-\mathrm{N} \& \mathrm{~S} 1 / 8$ tine; thence $\mathrm{S} 89^{*}-54^{*}-15^{m} \mathrm{~W}$, 
1114.03 feet along the South Section line; thence $N 00^{*} 12^{\prime}-15^{\prime \prime} \mathrm{W}, 176.00$ feet; thence $\$ 89^{*}$ $54^{\prime}-15^{\prime \prime} \mathrm{W}, 200.00$ feet to the point of beginning, containing 39.31 acres, more or less.

The second proposed site iocation is in Sagarting, Arenac County, Standish Township, section 30, north of Worth Rd. and west of the tracks of the Detroit and Mackinac Railroad line. The GIS coordinates for this site are (Longitude $43^{*} 55.625^{\prime} \mathrm{N}$ and Latitude $83^{*} 54.989^{*} \mathrm{~W}$ ). This site consists of 97 acres of tribally owmed property known as the Noffsinger site. (Maps included $\mathrm{N}-\mathrm{t}, \mathrm{N}-2$ )

Legal description: that part of the Southwest $1 / 4$ of the Southwest $1 / 4$ of Section $30, T 18 \mathrm{~N}-\mathrm{R} 5 \mathrm{E}$, Standish Township, Arenac County, Michigan, Jying West of the Detroit Mackinaw Raiłway right-of-way, and also a piece of land wits point of beginning located at the Southeast comer of the Southeast $1 / 4$ of the Southwest $1 / 4$ of Section 30, T18N-R5E, Standish Township, Arenac County, Michigan; thence North 80 rods; thence West 63 rods; thence South 80 rods; thence East 83 rods to the point of beginning.

\section{SEARCH AND RESEARCH OF MATERIALS, BOOKS AND MAPS:}

The Ziibiwing Center of Anishinabe Culture \& Lifeways has an extensive collection of Tribal research materials, historical and rare books and maps available for staff research. Some of the resources utilized for the research on these property sites were books. The following books were researched: The First People of Michigan by W.B. Hinsdale, Primitive Mon in Michigan by W.B. Hinsdale, Archeological Aflas of Michigon by W. B. Hinsdale, The Territory of Michigon by Alec R. Gilpen, Atlas of Great Lakes Indian History by Helen Hornbeck Tanner and Diba Jimooyung by the Saginaw Chippewa Indian Tribe of Michigan. The above authors did not refer to any historical or cultural significance to either of these two proposed sites. The Hinsdale maps from 1931 and USGS maps from 1973 were also utilized and showed no historical significance to these two proposed sites. (Maps included B-5, N-3) Included in our research was Appendix A, (List of Documented Cultural Resources in Arenac, Bay, Isabella, Midland and Saginaw Connties fron Andrews Cultural Resources. This report was prepared for the Saginaw Chippewa Indian Tribe of Micligan, March 19, 1997). This report addresses no historical or cultural significance to those proposed site areas. A review of Tribal records indicates that no previous archaeological surveys or cultural studies have been conducted within the proposed site areas. A physical site visit was conducted on each proposed site area and photos are included (B-8, N-7).

\section{ELDER CONSULTATION:}

Site 1, Mt. Pleasant, Michigan-Robin Spencer conducted an oral interview with Bea Colwell, a Tribal elder and Dr. Benjamin Ramirez Stikwegnaabi, PhD., Associate Professor of History at Central Michigan University, Mt. Pleasant, Michigan and community member. During the interview both could not remember or did not know of any historical or cultural significance such as burial sites, vilage sites, seasonal camp (hunting and gathering) or pre-reservation land use. Both remembered that the tribe now owns the property and that it was placed in trust. Don Seal, Community Engineer of the Planning Department for the Saginaw Chippewa Indian Tribe of Michigan also confirmed the fact that the property is in trust arid tribally owned. 
Site 2, Standish, Michigan- Anita Heard and Robin Spencer conducted an oral interview with a group of community members at the Saganing Out-teach center on June 16,2006 at their daily luncheon. During our interview no one recalled any historical or cultural significance such as burial sites, village sites, seasonal camp (hunting and gathering) or pre-reservation land use could not be recalled. To the best of their knowledge it had always been farm land. Anita Heard and Robin Spencer were told by Carol Tally, Assistant Director, and Don Nelson. Director of the Saganing Outreach Center, that located at the back of the proposed site area there was a creek with a huge rock that the natives once used to wash their clothes on. You can see the grooves or lines in the rock. (Photos included N-6)

The property adfacent to the proposed site area had much historical and cultural significance. There are Native cemeteries and burial grounds, a prominent trail, and lands where the Native families would have meetings and places they gathered and hunted.

\section{PAST USE OF THE PROPOSED SITE AREA:}

Before ca. 1580: This period is marked primarily by the fact that the Ojibwa have not yes had contact with Europeans. Culture is centered on hutting, fishing. gathering and planting trade. Setlements are small and seasonat with some larger intertibal gatherings for ceremoniat purposes. The material culture does not yet have items of European origin.

Ca. I580-1650: Period of inirial contact with Europeans primarily though their goods being obtained in trade with other tribes. Some pandemic disease episodes may have occurred. First face to face contact with Europeans occurs ca. 16/5 with Champlain arriving along the east shore of Lake Huron. Ojibuas cthiture is still centeret arowd hunting, fishing and gathering.

Ca. 1650-1830: Period of sustained contact with Europeans following the dislocation and destruction of many ribes as a result of the Iroquois wars and pandemic disedse episodes. The period is marked by a greater amoun of European objects being introduced into Ojibwa Vifestyles.

Ca. 1830-1900: Period of intense pressure upon Ojibu'a culture as a result of large numbers of Euro-Americans migrating into their homeland. The period is marked by conversions to Christianity; changes in subsistence activities due to the destruction of the ecosystems by such things as logging and farming; changes fo log and wood frame housing; relocation to other areas; indoctrination of Ojibwa children with Euro-American culture through government and private (religious) schools. (Taken from "A Cultural Resources Inventory Project Report prepared by Andrews Cultural Resources)

In the preliminary physical site visit of Benzinger Il, Robin Spencet and Anita Heard walked directly ftom the east side of Shepherd road out to the test tower. In our brief assessment we noted the following plants and their typical cultural usefulness. ( see append. B-8)

In review of the map entitled: Vegetation cinca 1800 of Isabella County, Michigan, and interpretation of the General Land Office Surveys, produced by Michigan Department of Natural Resources, Michigan State University Extension Office and Michigan Natural Features 
Inventory, the Benzinger If Site was a hemlock - white pine forest bordering a beech and sugar maple forest. The resources offered in both of these environunents would have provided a great many typical foods and medicines. The same map source as indicated above entitled: Land Coverage Change, 1800's -1978 , Isabella County states the most recent survey shows charge to aspen bordering urban and agriculture areas, which is consistent with our physical site review.

Remaining consistent with the review of archival and historic preservation documentation we found no indication of significant past use of this property even though a branch of the Onion Creek is noted on several maps to begin on this property, no evidence was noticed. The history of this general area has been addressed below as this property lies with in the six township border of the historical reservation as defined in the 1855 Treaty of Saginaw.

In the physical visit of the Noffsinger site Robin Spelwer and Anita Heard, walked the east border (tailroad tracks) and the north border of parcel 3. Noted were the following plants and their typical cultural usefulness. (see append. N-7)

In this assessment it was exceptional the number and quantity of food source plants. In light of the plant materials evidenced, this general area most likely was an important site for gathering food and fiber materials. This would indicate a prime location for a settiement or seasonal camping area.

To support this theory a foot path well known to the community had run along the shoreline which is located approximately four miles east of this site. This trajl comes off of the Mackinaw Saginaw Trail at Saginaw (cited in Hinsdale's Michigan Archaeological Atlas, map numbers 9, 12, and 14 and figure 1 ). Recent interviews of community members revealed that the Saganing River which borders this property on the northern side was very important. In the past it not only provided fishing, fresh water, and a water way for travel but had been abundant with wild rice, a staple food of the Ojibwa. The river was also mentioned several times with a reference to a "washing rock". We were given the general location and did find it. (See photo N-6) The interviews also lead us to two cemetery sites with few stones. lt has not been until recent times that markers were set for graves. The early dates observed where mid- $1800^{r} \mathrm{~s}$.

In review of the map entitled Vegetation circa 1800 of Arenac County, Michigan and interpretation of the General Land Office Surveys, produced by Michigan Department of Natural Resources, Michigan State Univerșity Extension Office and Michigan Natural Features Inventory, the Noffsinger Site was a cedar swamp bordering an aspen-birch forest. The resources offered in both of these environments would have provided a significant cultural resource. Tliese environments would have produced a great many typical foods. medicines and housing materials. The same map source entitled Land Coverage Change, 1800's -1978. Arenac County states the most recent survey shows change to urban and agriculture areas, which is consistent with our physical site review.

It has been confimed through our research, elder consultations and physical inspection that the only recent past use of both proposed site properties was farming or just left to grow. 


\section{ISABELLA COUNTY RESERVATION:}

The teservation located within Isabella County was created in 1855 by the Treaty with the Chippewa of Saginaw, Swan Creek, Black River and the United States; it is located within the present-day townships of Chippewa, Deerfield, Denver, Isabella and Nottawa, Union, Wise. The number of cuttural resources within the reservation and general area of Isabelfa County increases after ca. 1855 when members of various bands of the Saginaw, Swan Creek and Black River Chippewa's chose to relocate there. (Portions taken from "A Cultural Resources Inventory Ptoject Report prepared by Andrews Cultural Resources)

\section{ARENAC COUNTY RESERVATION (SAGANING):}

No previously recorded sites are known for tle present-day land associated with the original Saganing Reservation. The reservation has been occupied by a band affiliated with the Saginaw, Swan Creek and Black River Chippewa since at least the early nineteenth century. It is krown that cultural resources associated with that occupation exist within and near the reservation however they have not been systematically identified or assessed. (Portions taken from "A Cultural Resources Inventory Project Report prepared by Andrews Cultural Resources) 


\section{Recommendations:}

Regarding the Benzinger II Site and the historic and archival references, piacing a tower in the same spot the test tower is located would pose no threat to cultural resources. Chances that plants found on the endangered, protected or special consideration plant list identified by the State of Michigan, could be found at this site would appear minimal. No recommendation to discontinue plans of construction.

Regarding the Noffsinger Site and the archaeological references, placing a tower in the same spot the test tower is located would pose no threat to any cultural resontces. However awareness and further investigation should be under taken if the project moves forward because there were several plants which could possibly be found on the endangered, protected or special consideration plant list identified by the State of Michigan. No recommendation to stop construction but a recommendation of identification and awareness of the area of habitat should be noted and avoided. 


\section{B-1}

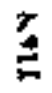
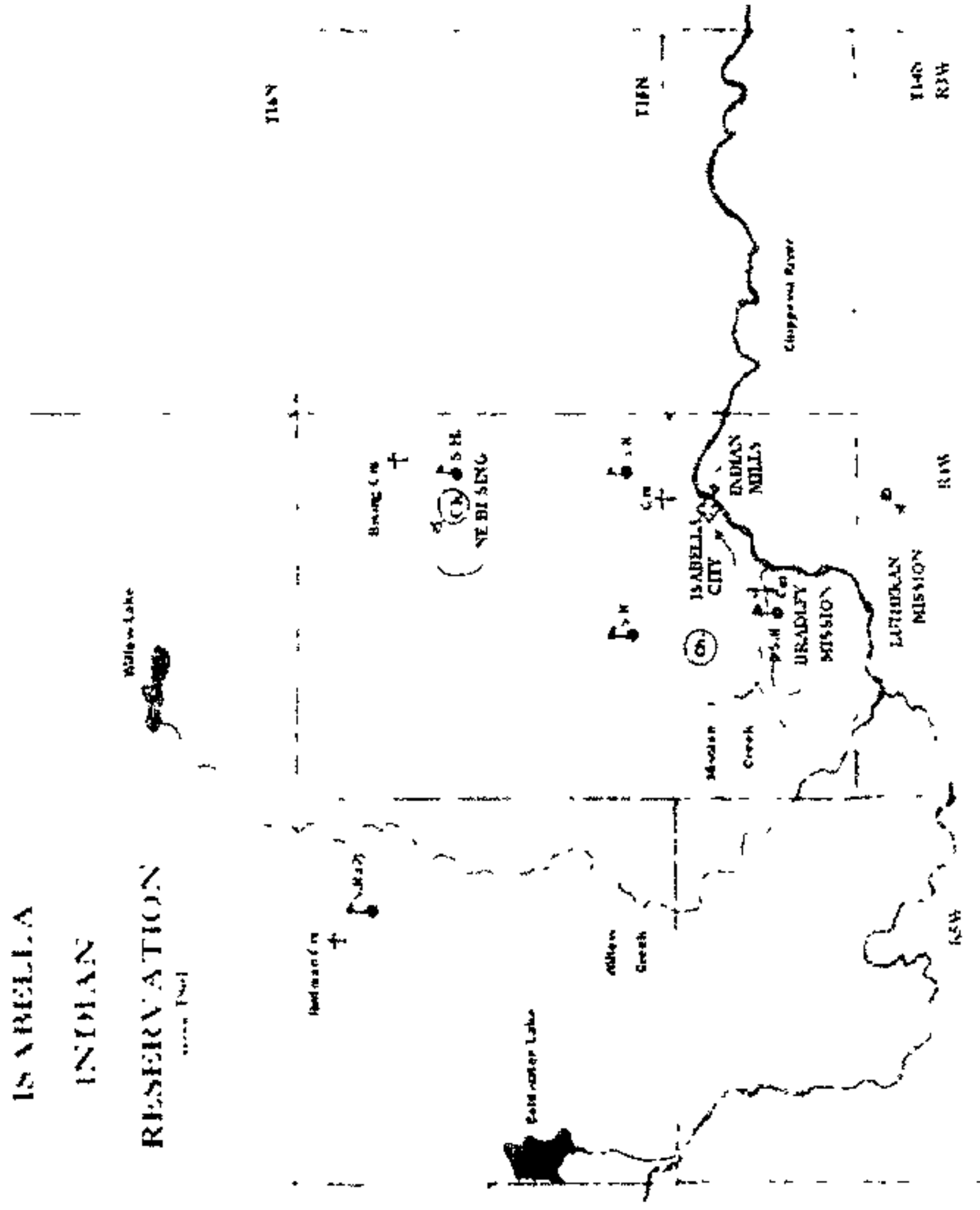


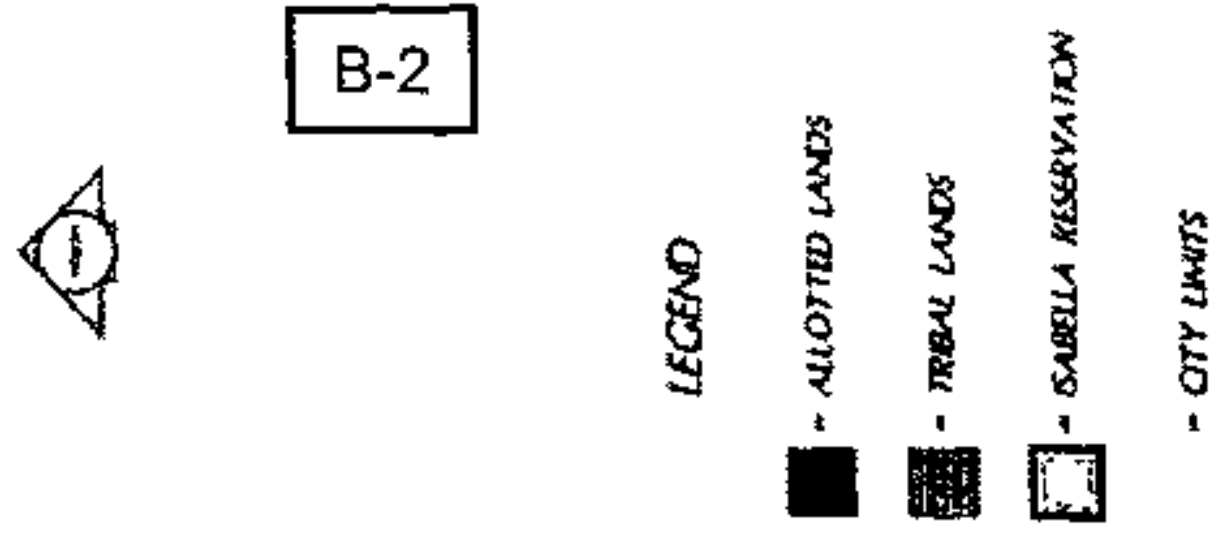

党:

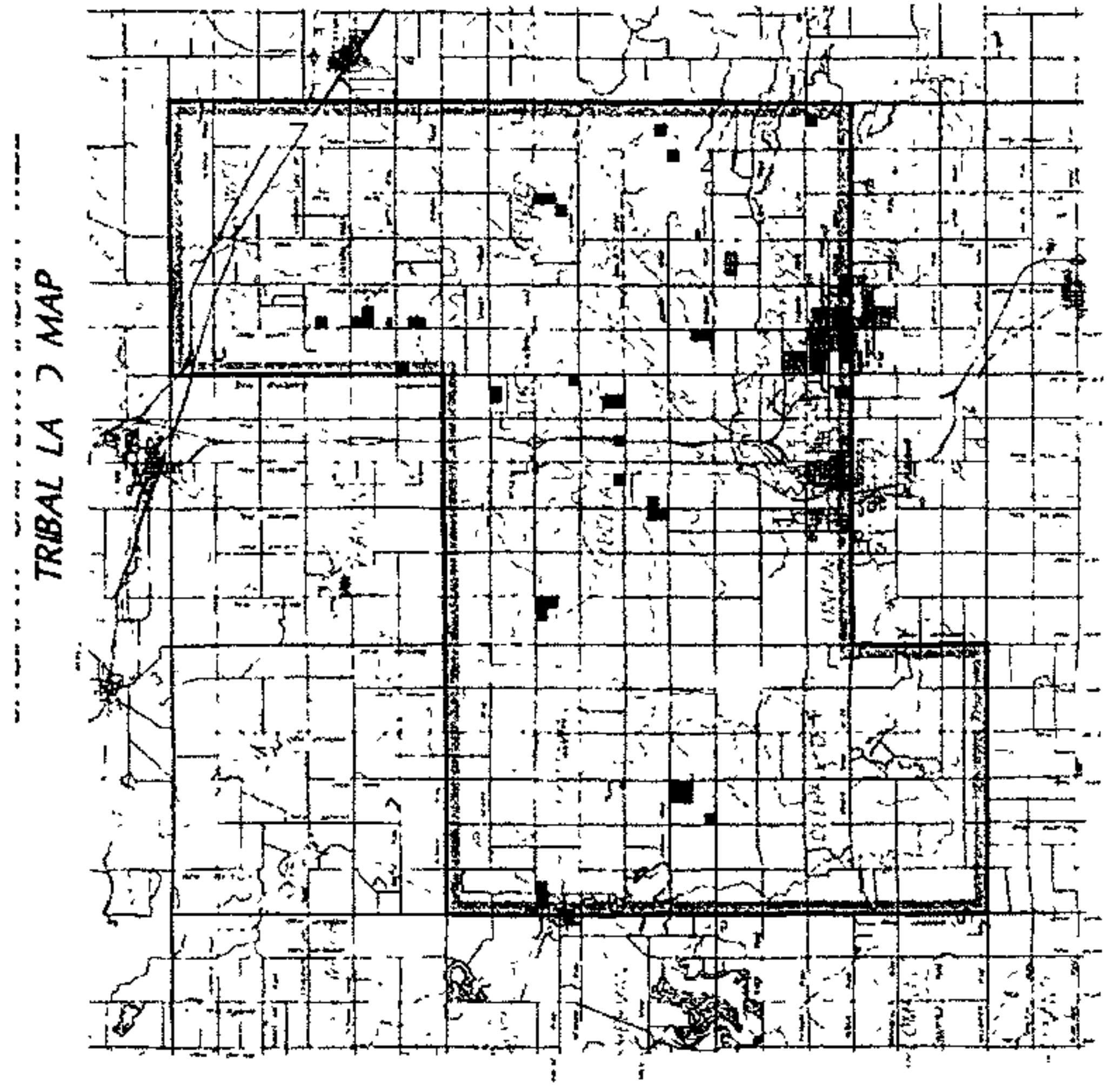




\section{B-3}

要

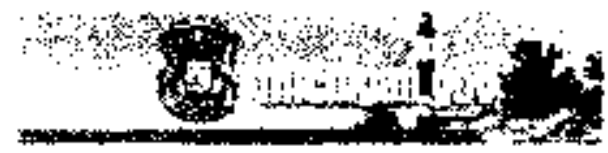

\section{Michigan \\ Gov Home \\ ingeres is}

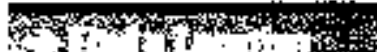

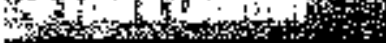

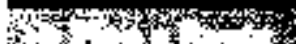

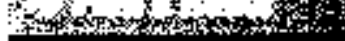

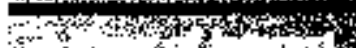

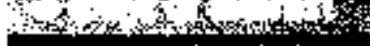

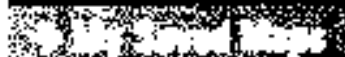

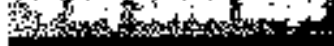

\section{$\Rightarrow$ Bromse the Nap}

\section{s.}

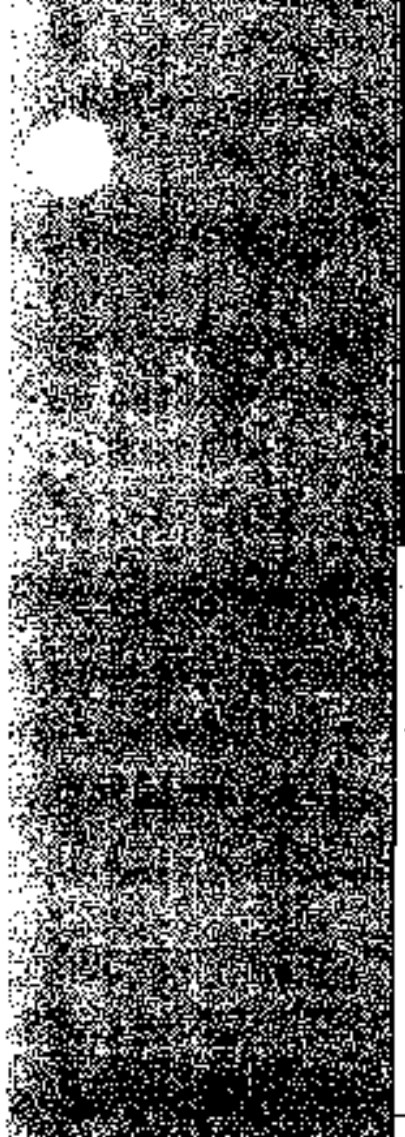

\section{Map $\frac{\text { Michigan }}{\text { Horne }}$ FAQ Help $\frac{\text { Contact }}{\text { CG! }}$}

\section{A MAP MICHIGAN}

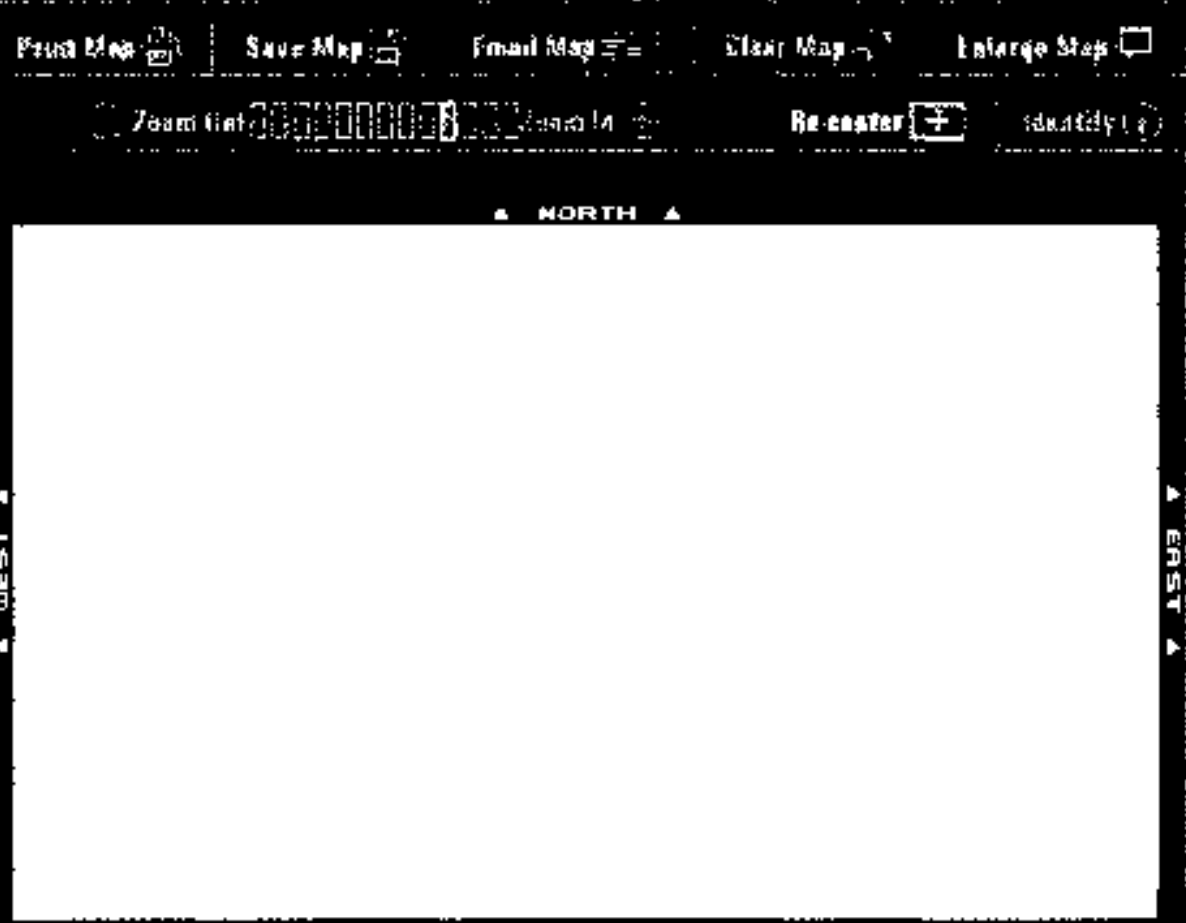

$\rightarrow$ sguTh_?

7.

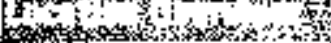
Intann

al at: 43.599183

: Lon: 84.686550

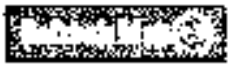

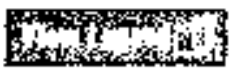

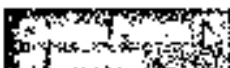

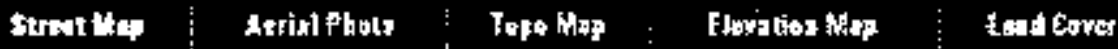

Michigangov Home / Site Map I State Web Sites Contact Michigan $\mathrm{EAQ}$

Privacy Policy L Link Policy I Accessibility Policy | Security Policy Copyright @2001-2003 State of Michigan 


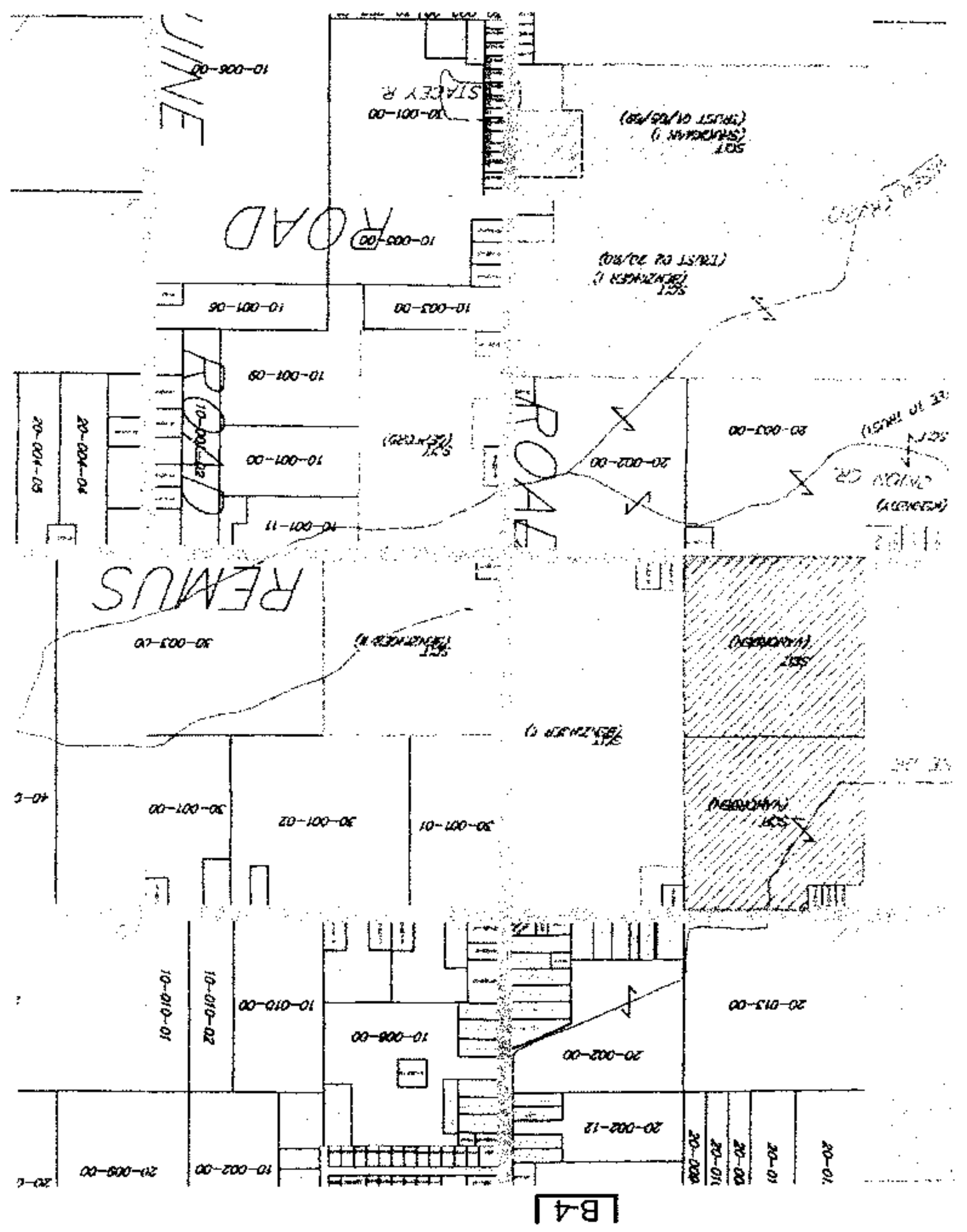




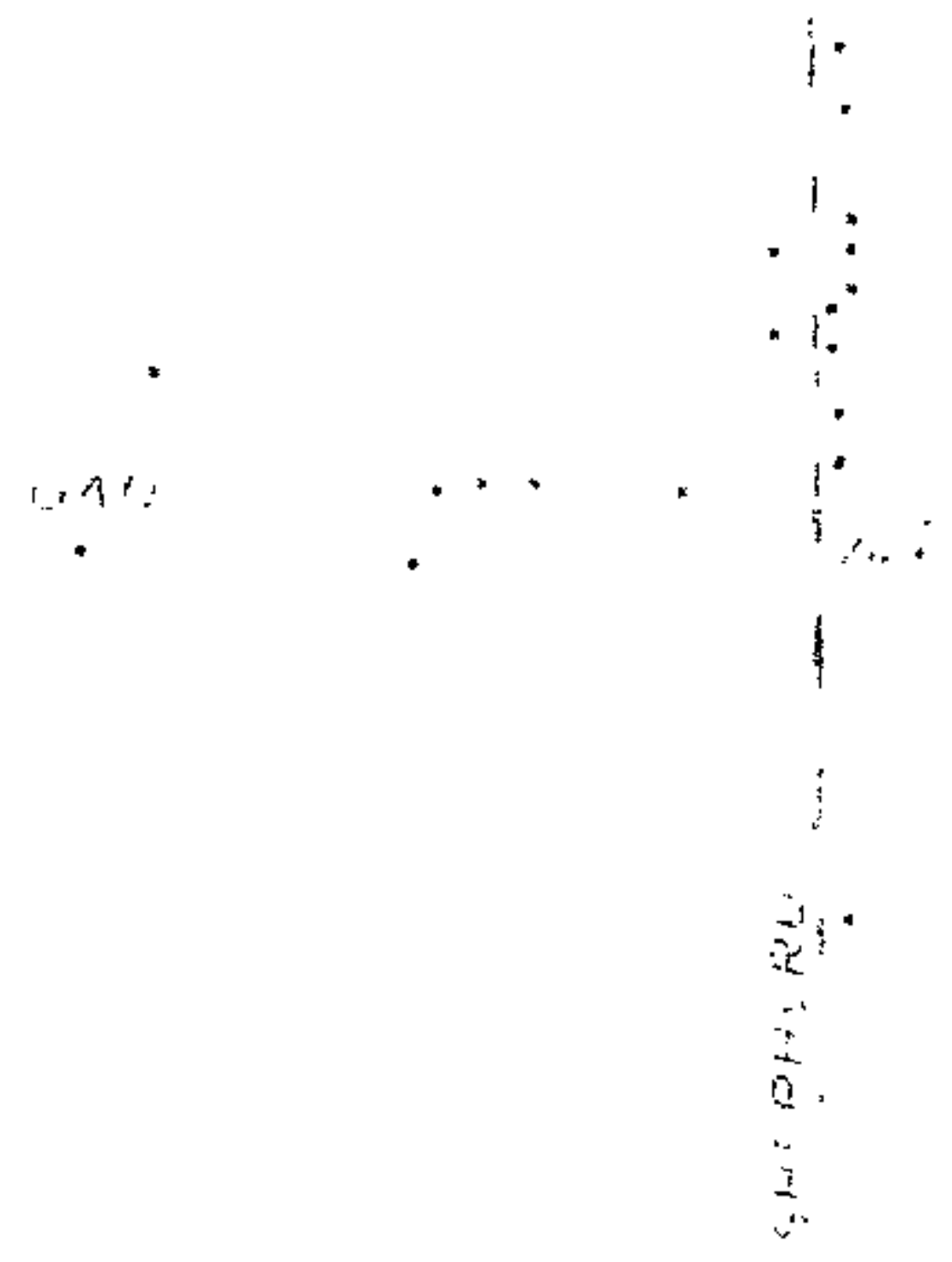

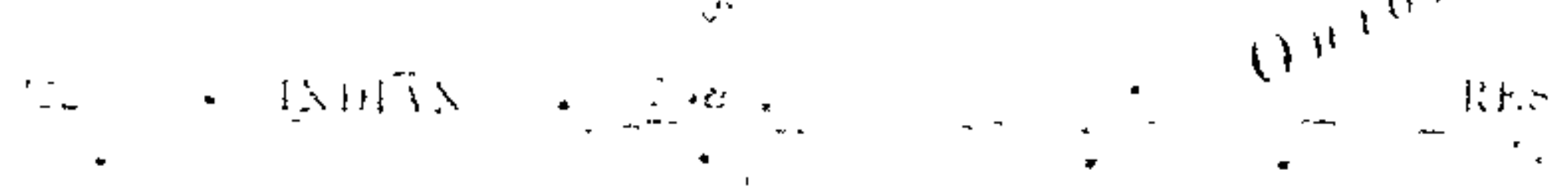

$\therefore$.
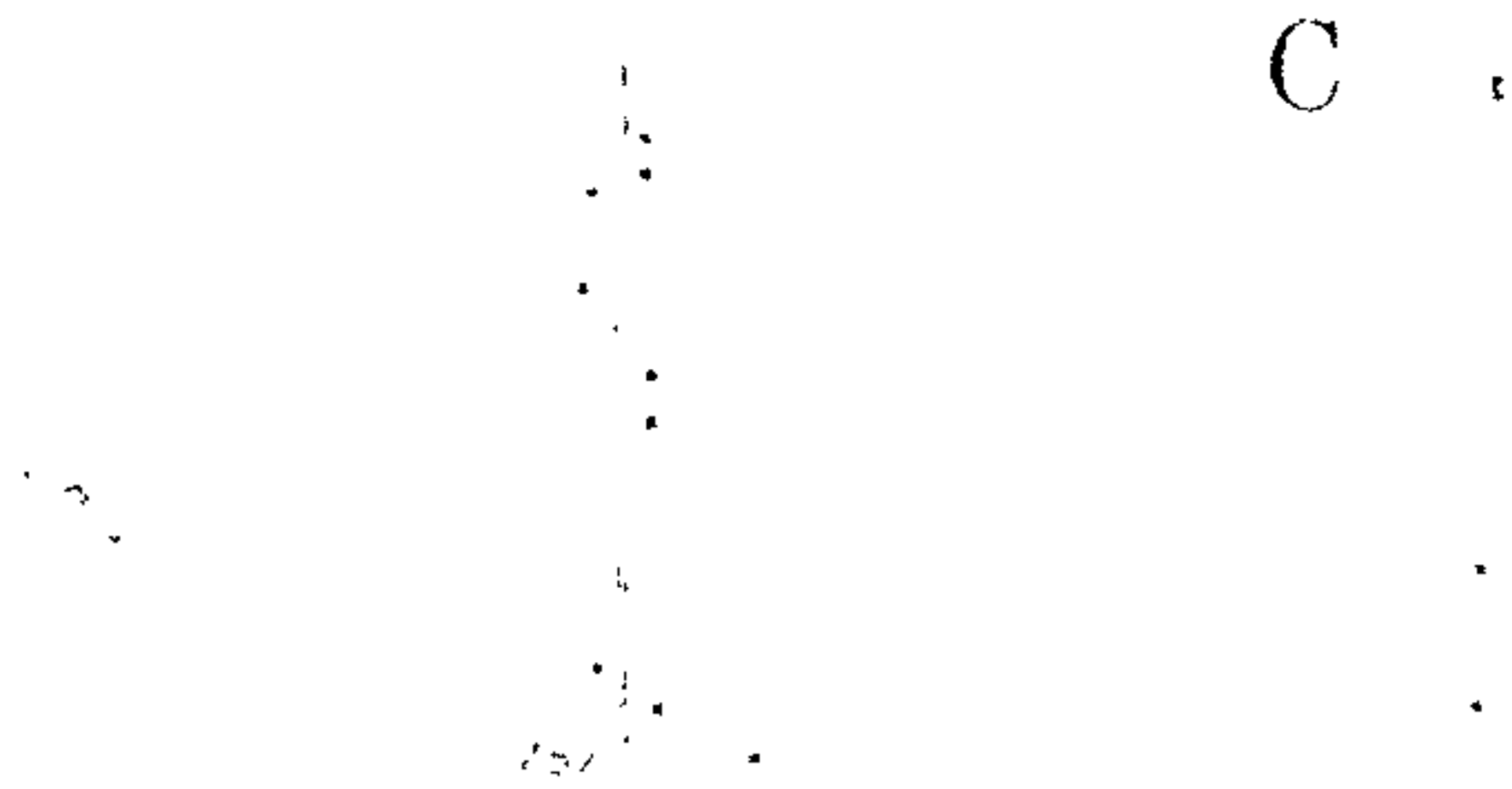


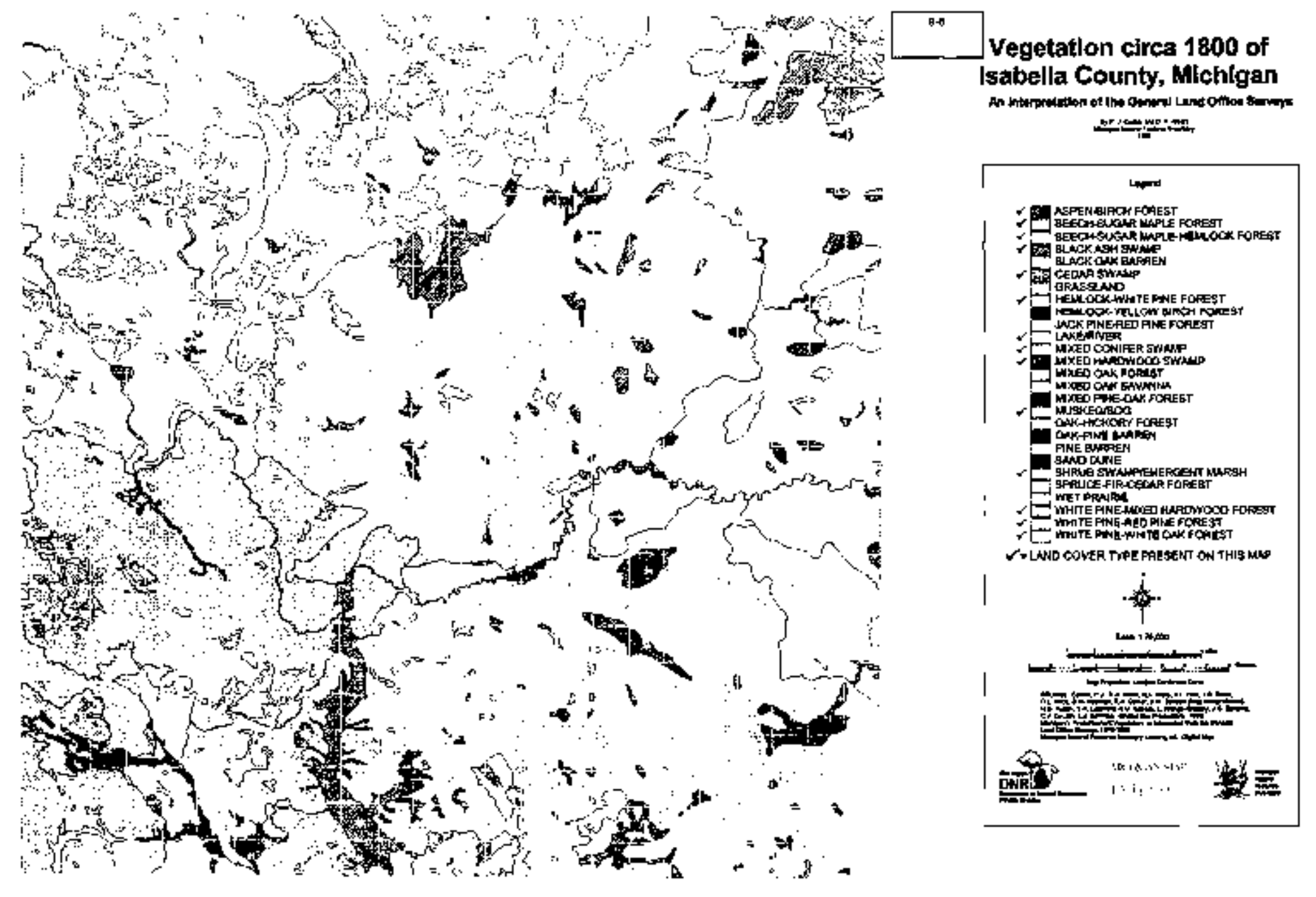




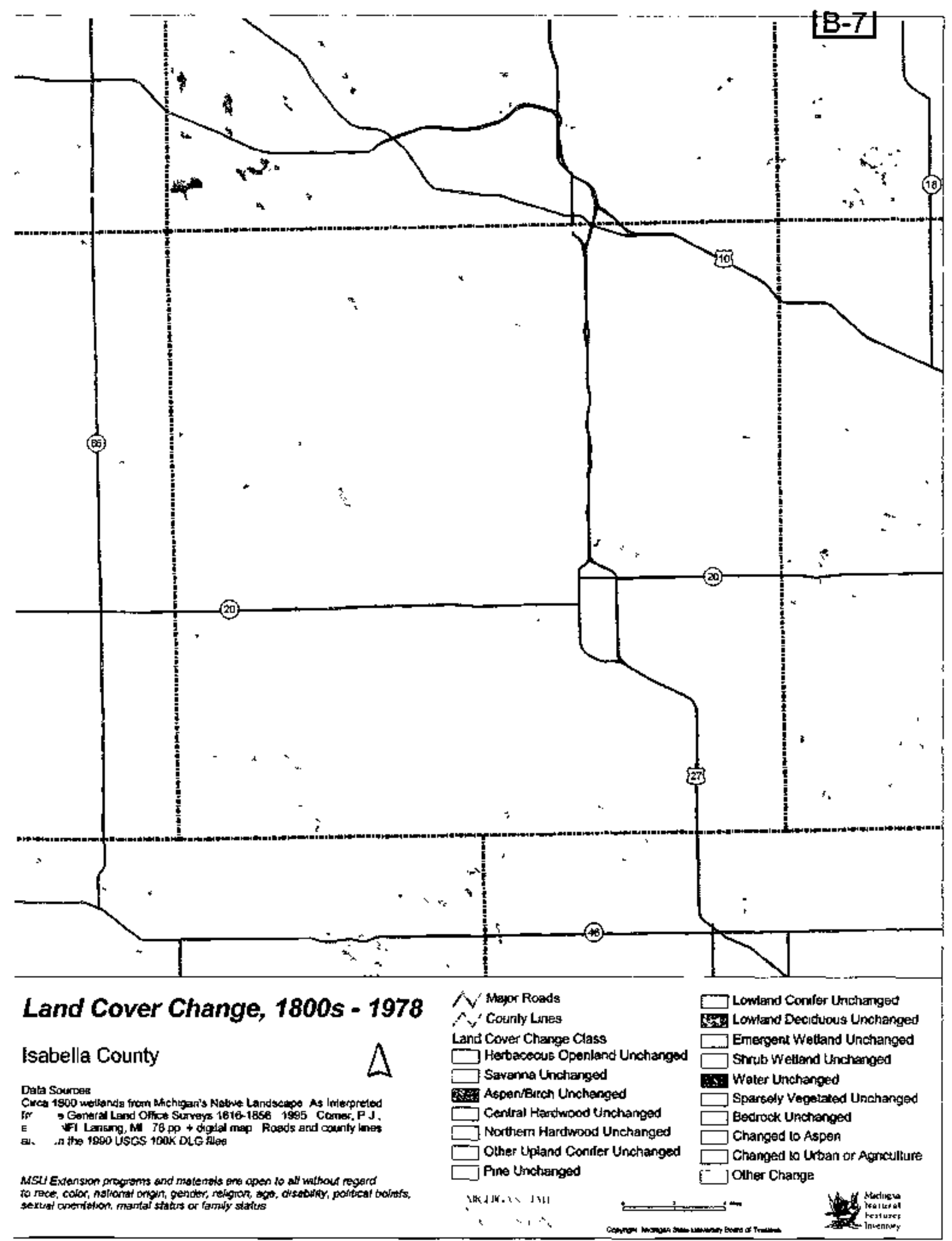




\section{B-8.1}

\section{Benzinger II Site Plant Sample}

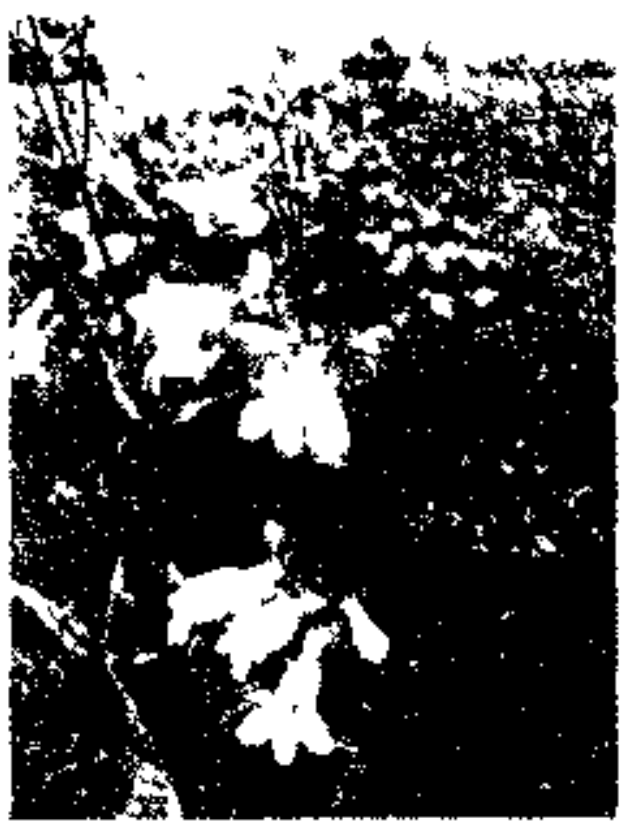

Beard tongue, foxglove beardtongue, tall white beardtongue (Penstemon digitalis) uncommon to tlye area

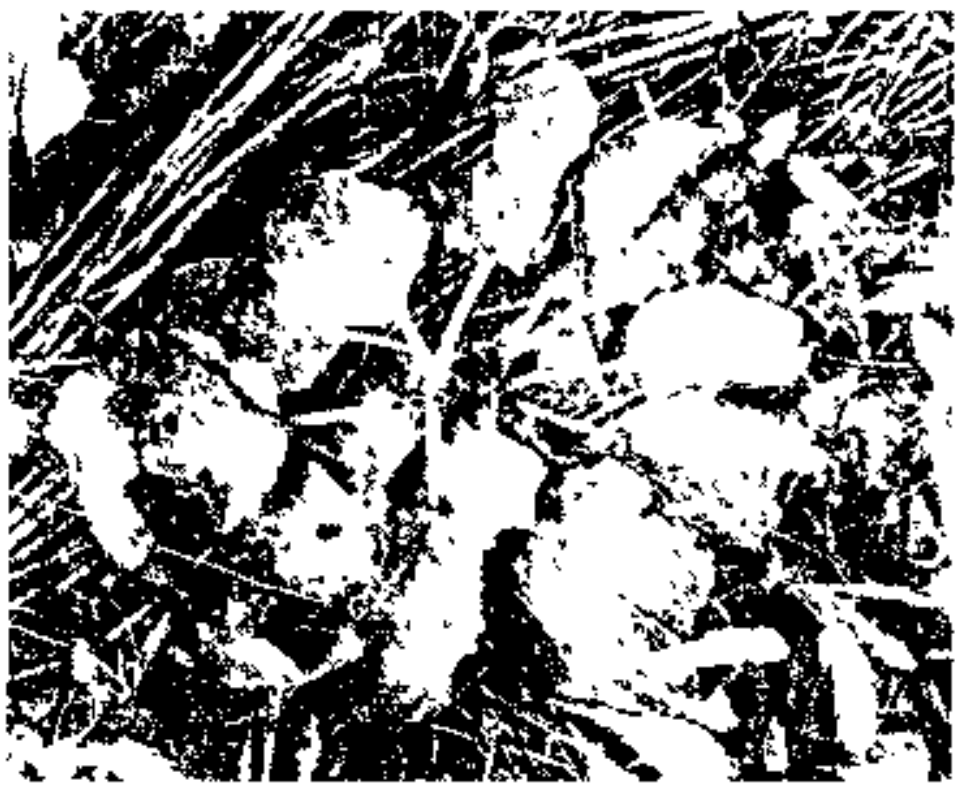

Broad leaf plantain, common plantajn (Plantago major $L$ )- useful in the treatment of insect stungs and bites, poultice for theumatism, inflammation and swelling, known to be antrbacterial, poultice for snake bite, the root used as snake bite protection medtcine, and provides food as greens 


\section{B-8.2}

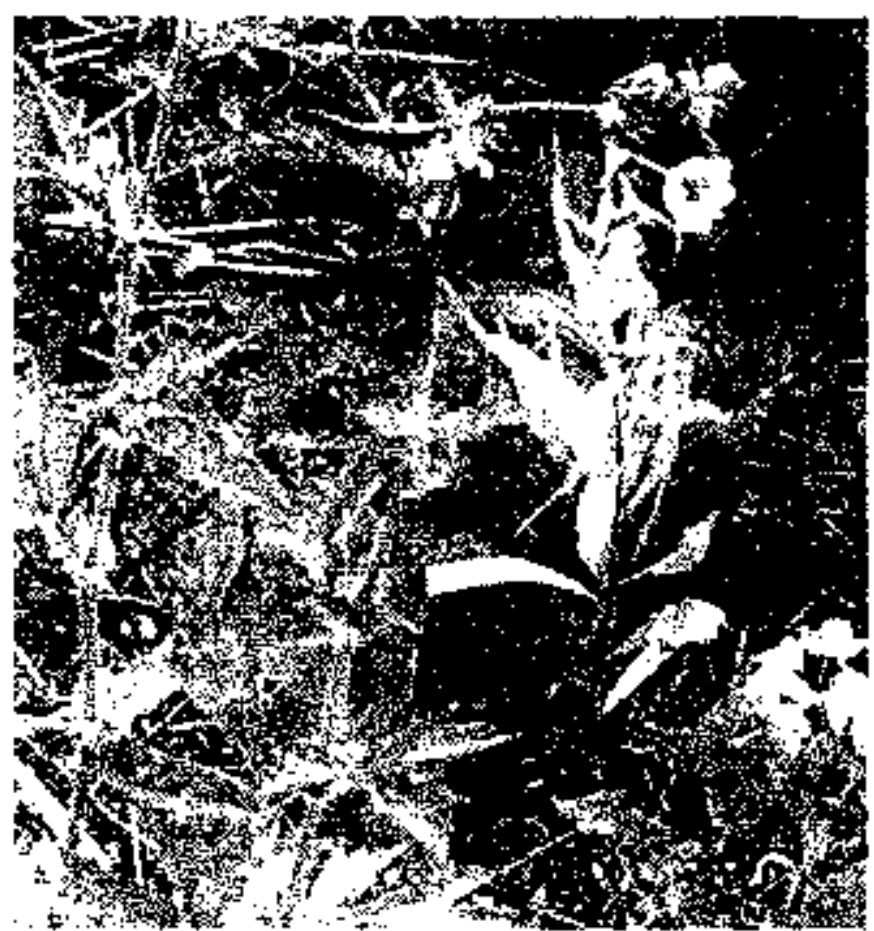

Butter cup, Meadow Buttercup, Common Buttercup (Ranuncults acris) useful as colnter irritant for arthritis, seeds used for hunting medicine, plant cornbined with oak for red dye, and combined with flag leaf for yellow dye

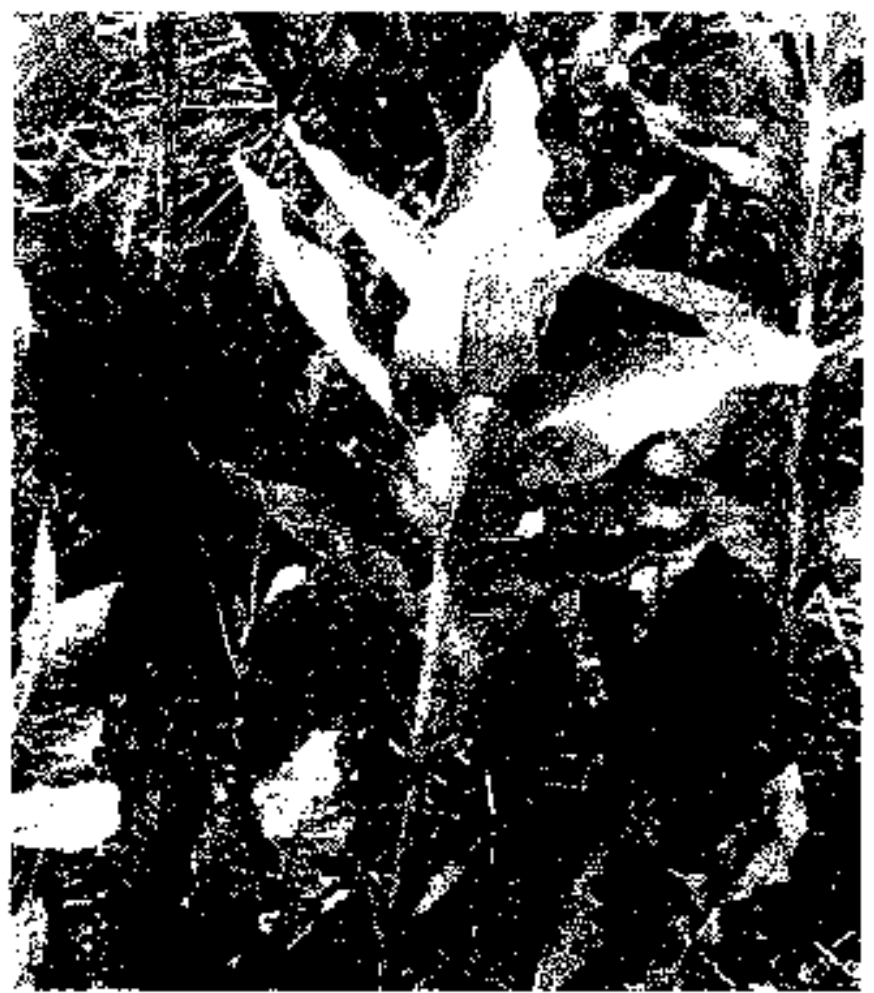

Common Milkweed (Asclepias syriaca L. Asclepiadaceae) the sap is useful in combating warts, the root useful in the treatment of female problems, the flowers, young shoots and seed pods an excellent food source 


\section{B-8.3}

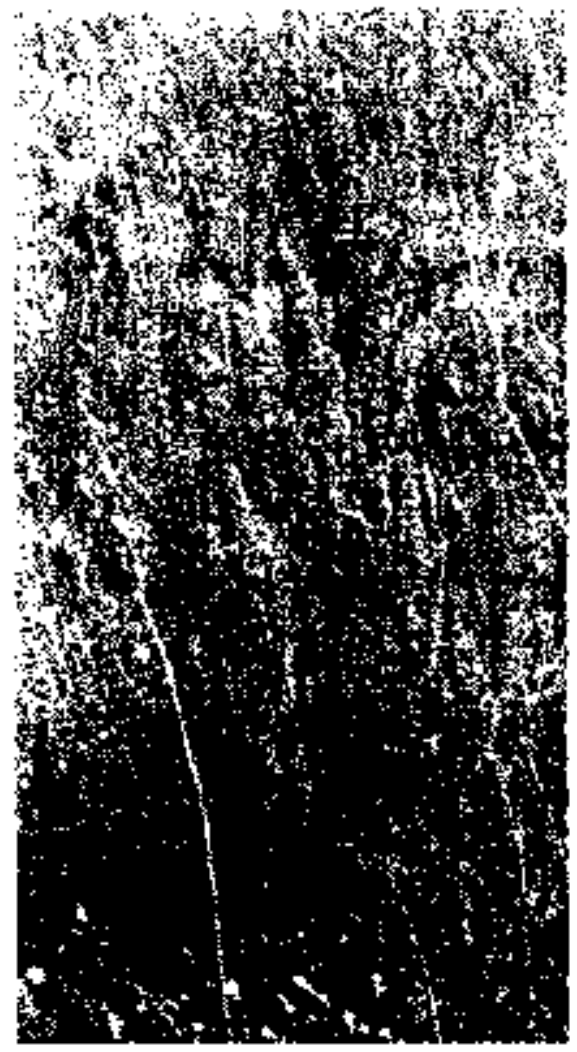

Curly Dock (Rumex crispus L. Polygonaceae) Poultice of moistened, dried, powdered root applied to cuts or itches.

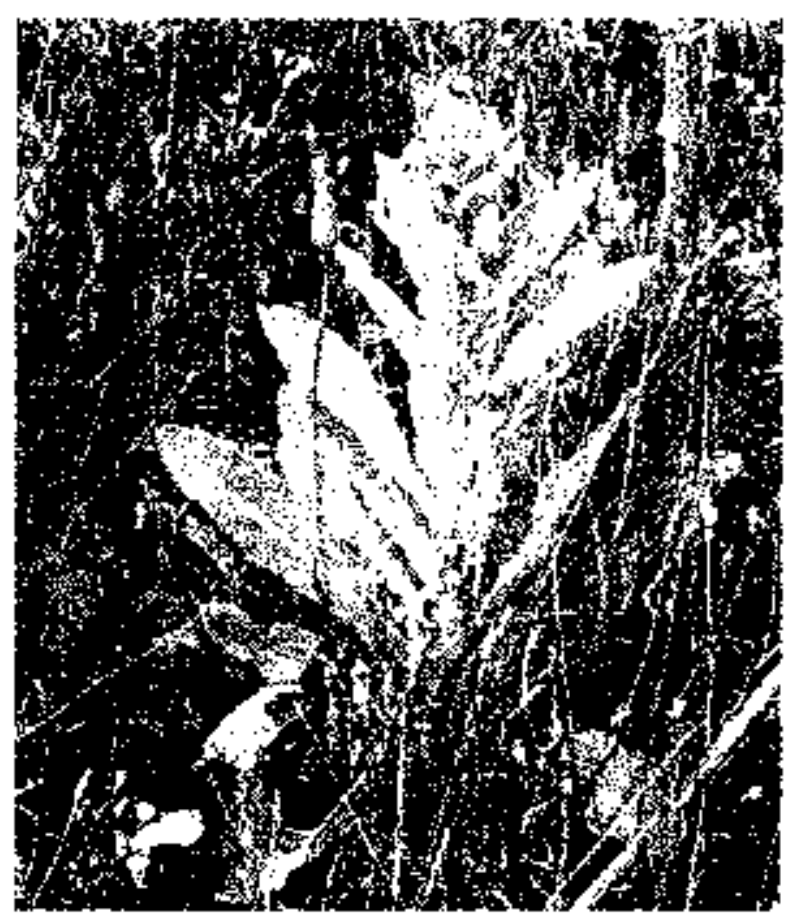

Mullein, common muflein (Verbascum thapsus L.) upper portion useful in treatment of respiratory illness, root used as a heart stimulant 


\section{B-8.4}

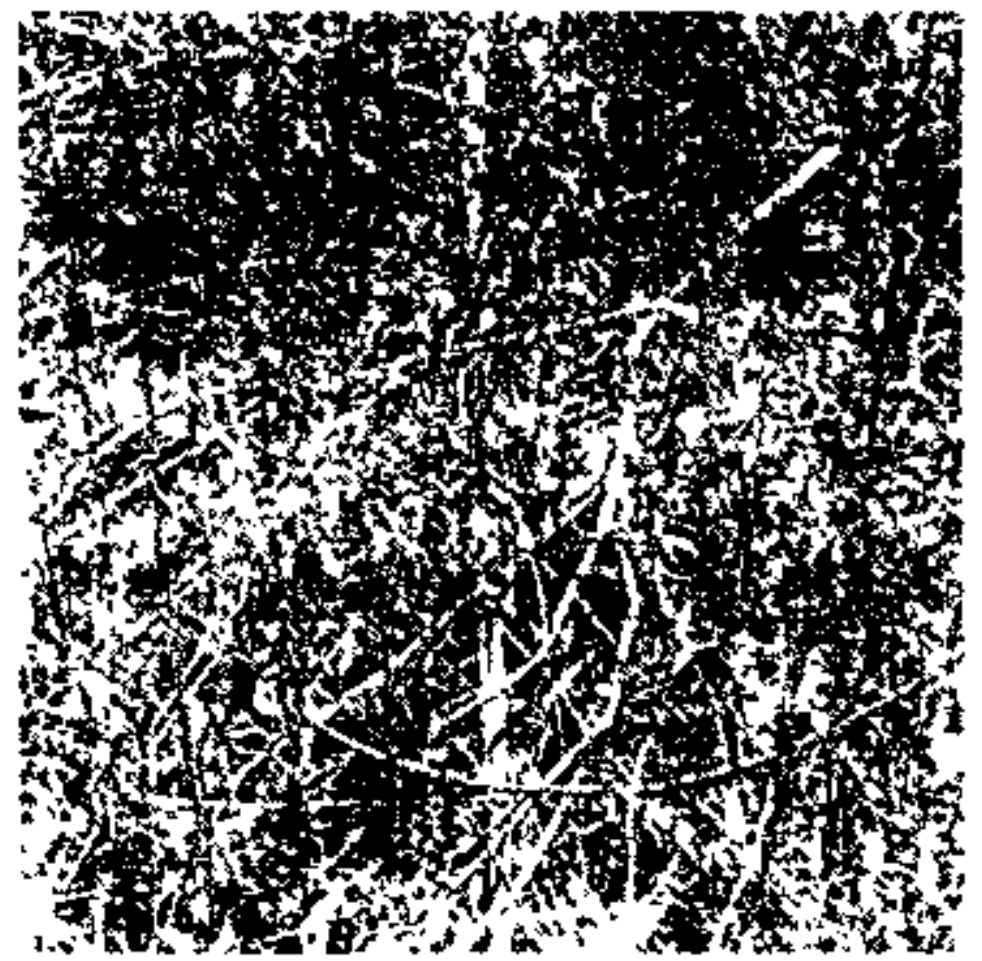

Sheep Sorrel (Rumex acetosella L) Vitamin C, sprng tonic, greetus

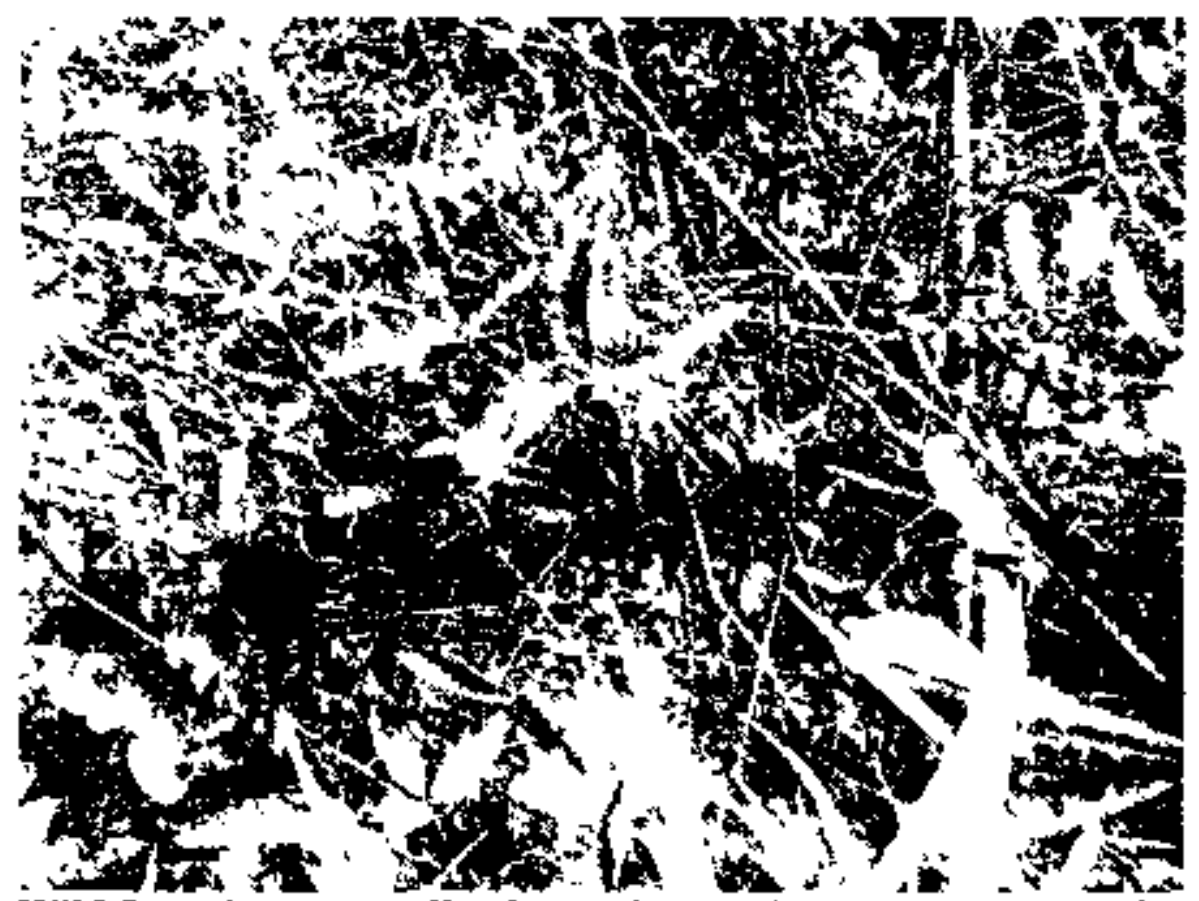

Wild Strawberry, woodland strawberry (Fragara vesca $L$ ) leaf and berry used for

Vıtamus C, spring tonic, urinary illness, useful tn ceremonial context 


\section{B-8.5}

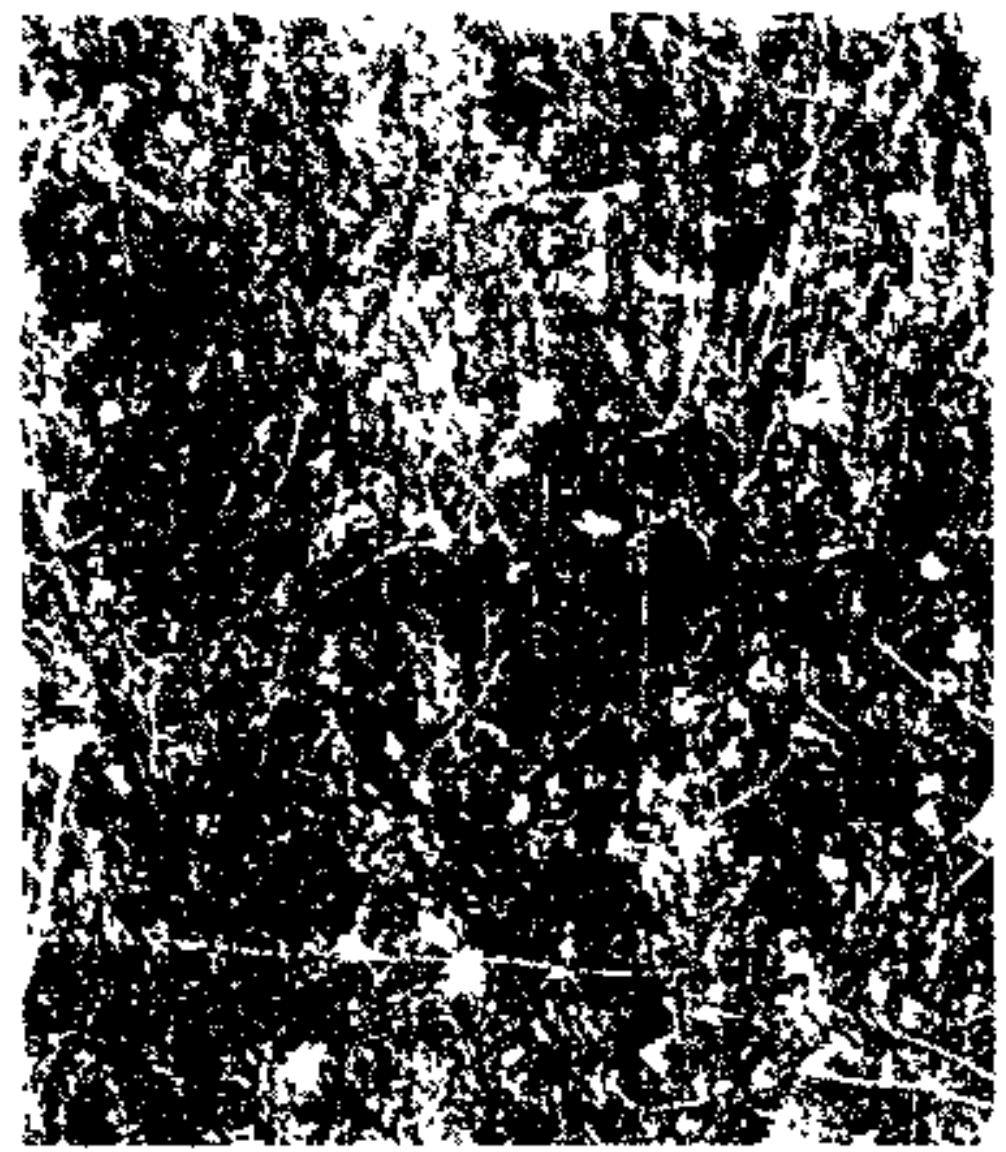

Yellow Hawkweed (Hieractm, fendlew Schwh-Bip Asteraceae) Hanting and fishing medicine

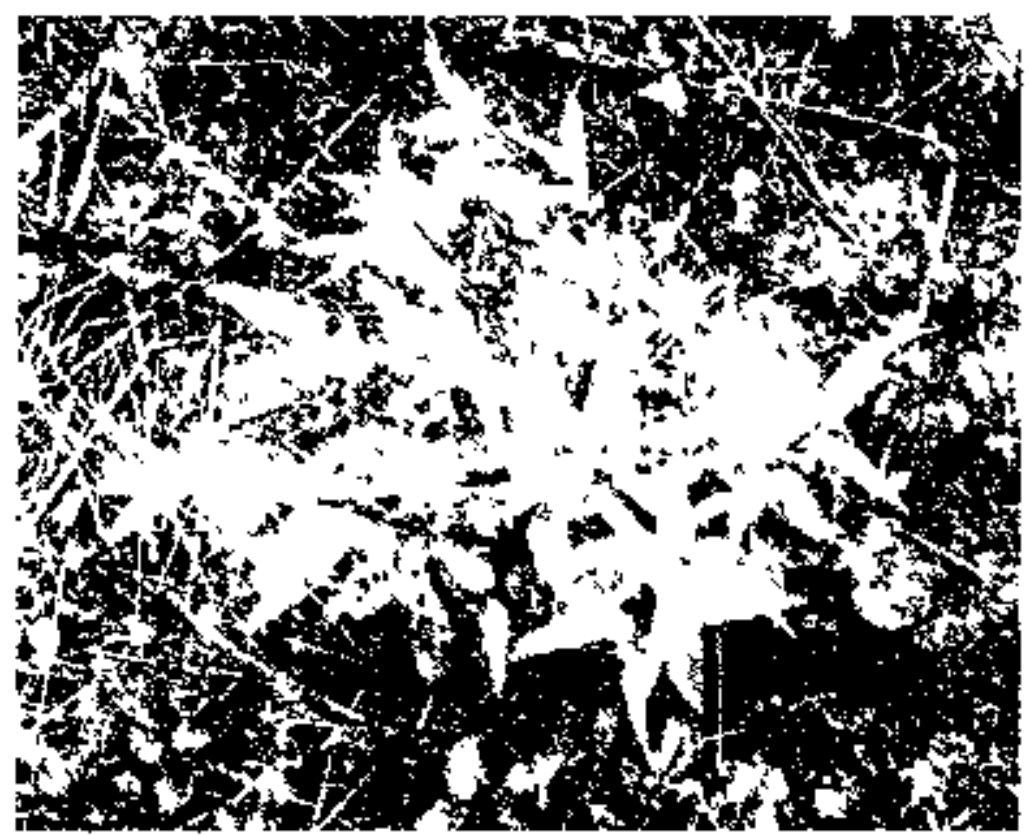

Red Osıer Dogwood (Cornus sericea $L$ ), inner bark used for ceremonial pipe smoking and offerings- Ingredıent of Kinnıkinnick, twigs used for basket making 


\section{B-8.6}

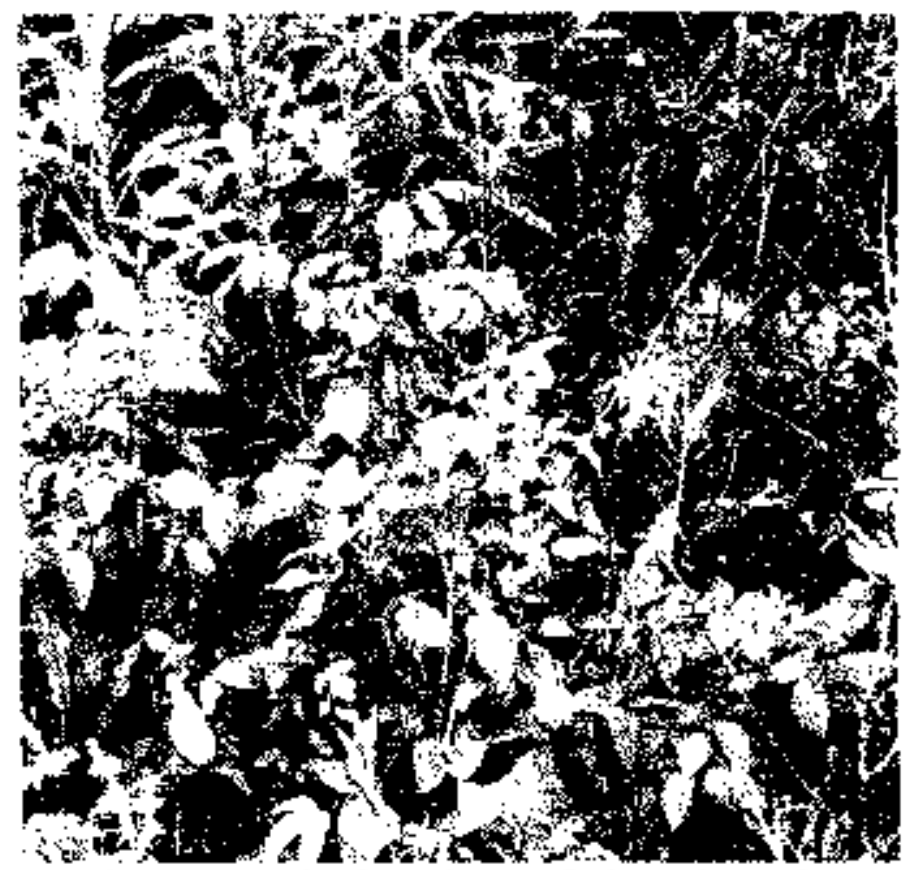

Raspberry, grey leaf raspberry (Rubus idaeus L. s.sp. Strigosus (Michx.) Focke) Food, anti-diarrhea, back pain. eye wash, treatment for measles

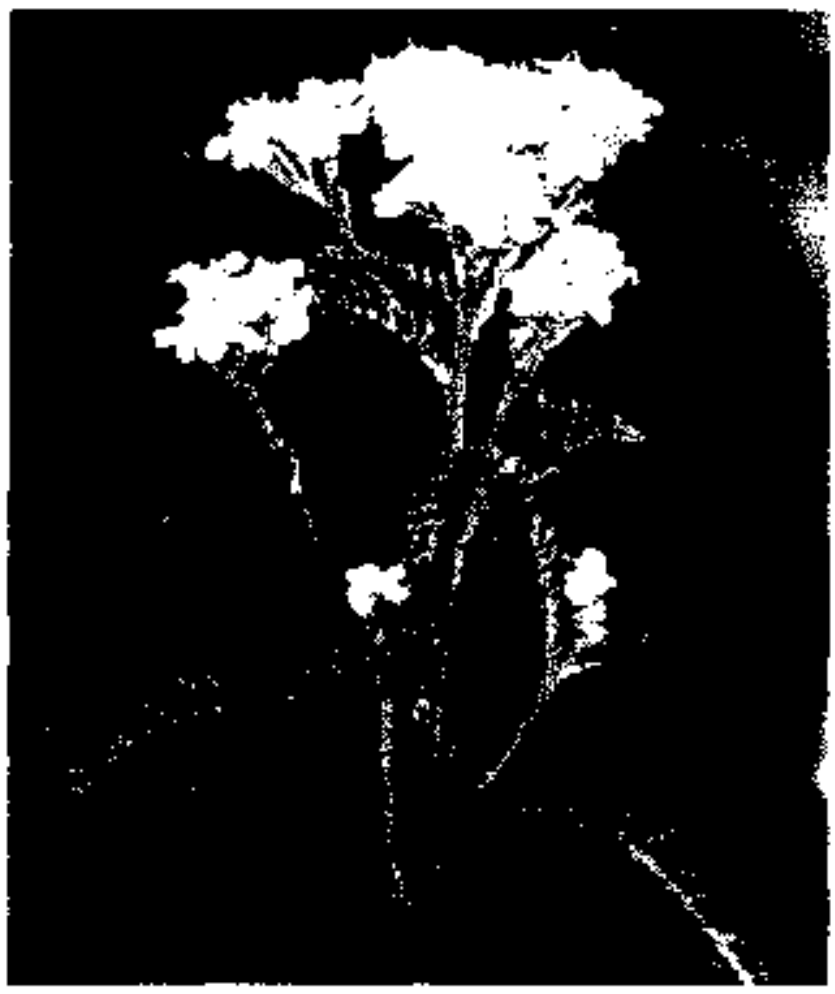

Yarrow, Milfoil, Thousand-leaf, Arrow-root, Bloodwort, Greenarrow, Soldier"s Woundwort, Nosebleed Weed, Devil's Nettle (Achiflea millefolium) Leaves useful as insect repellant, stop bleeding, digestive tonic, wash infected wounds, eye wash, reduce blood pressure, tooth ache and protection medicine 


\section{B-8.7}

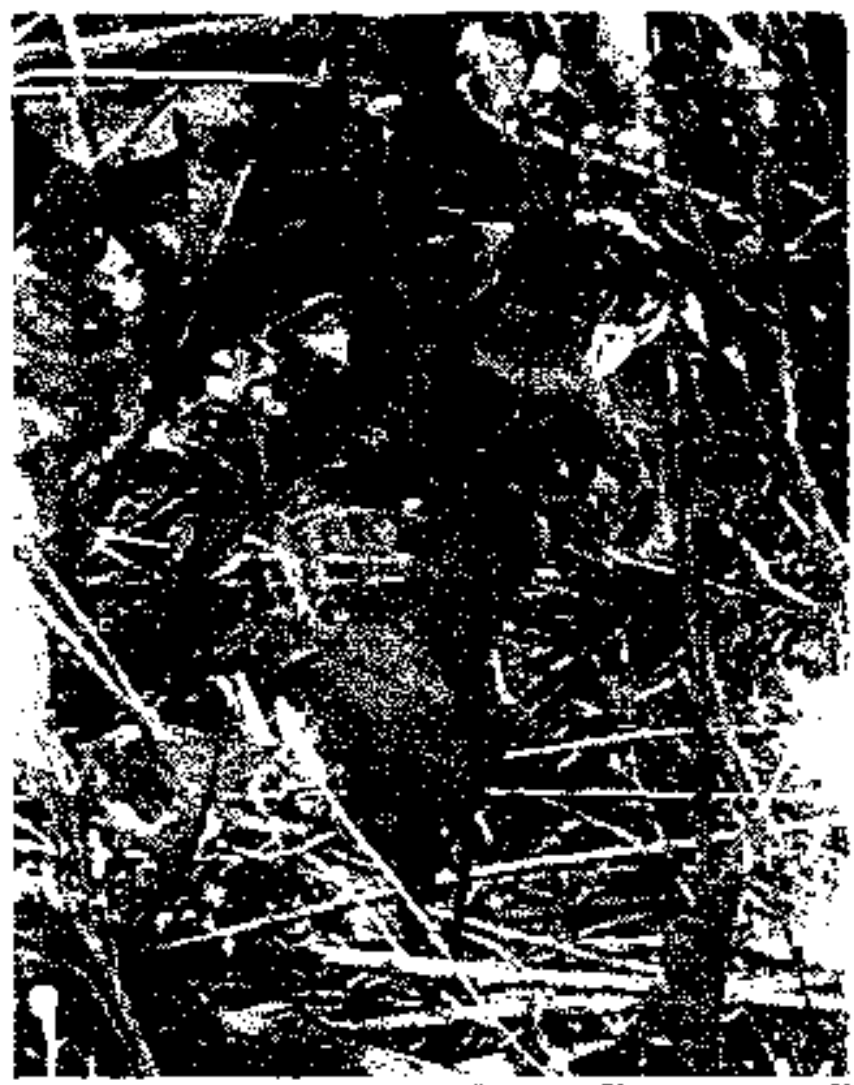

Dandelion, Conmon dandelion (Taraxacum officinale) spring tonic, blood purifier, greens

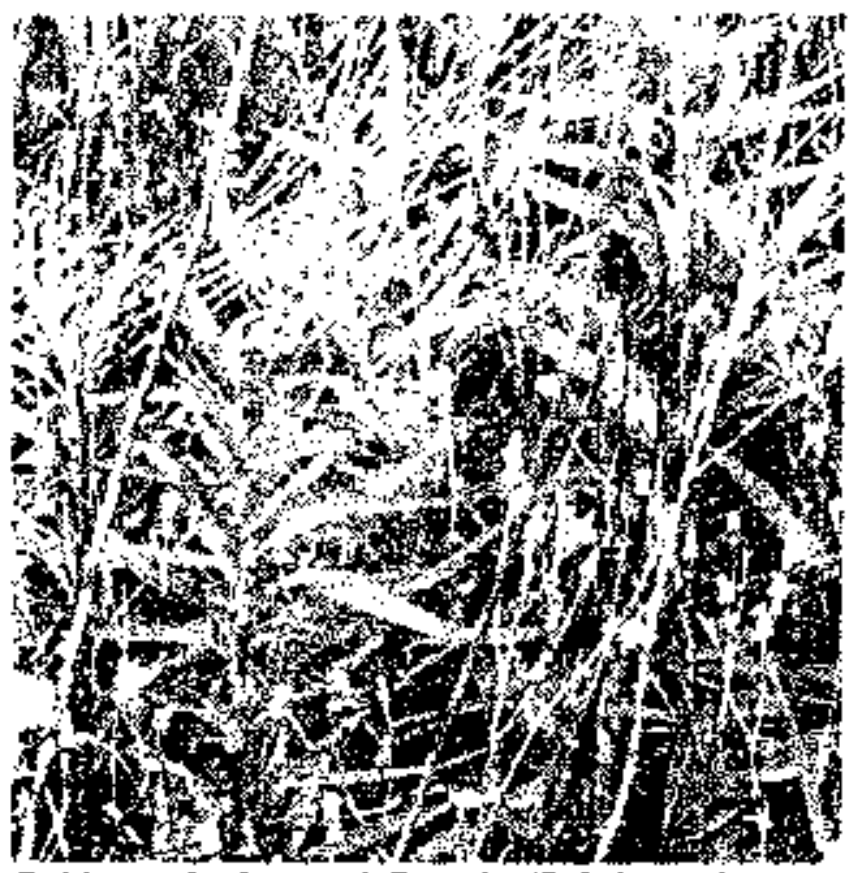

Golden rod - late and Canada (Solidago altissima L. and Salidago canadesis L.) Sore throat, flower used as a poultice for burns and boils, treatment for convulsions, cramps, fever and stomach aclue 


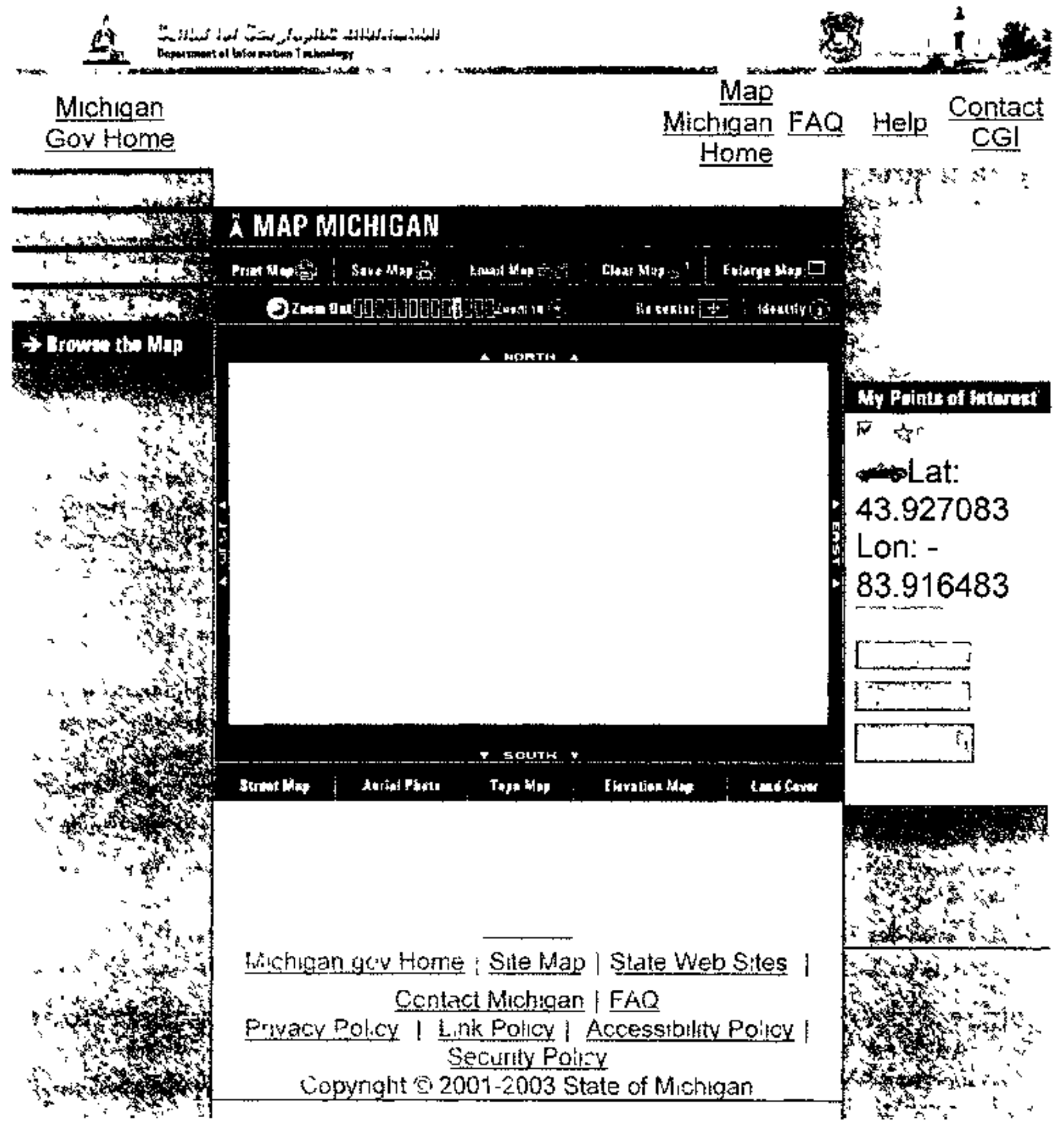




$$
\text { N-2 }
$$

SAGINAW CHIPPEWA INDIAN TRIBE SAGANING BASE MAP

sartsontin

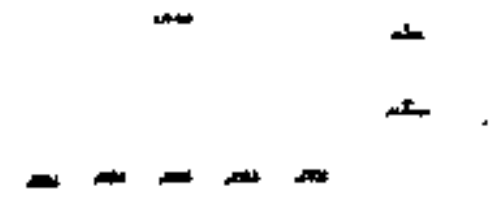



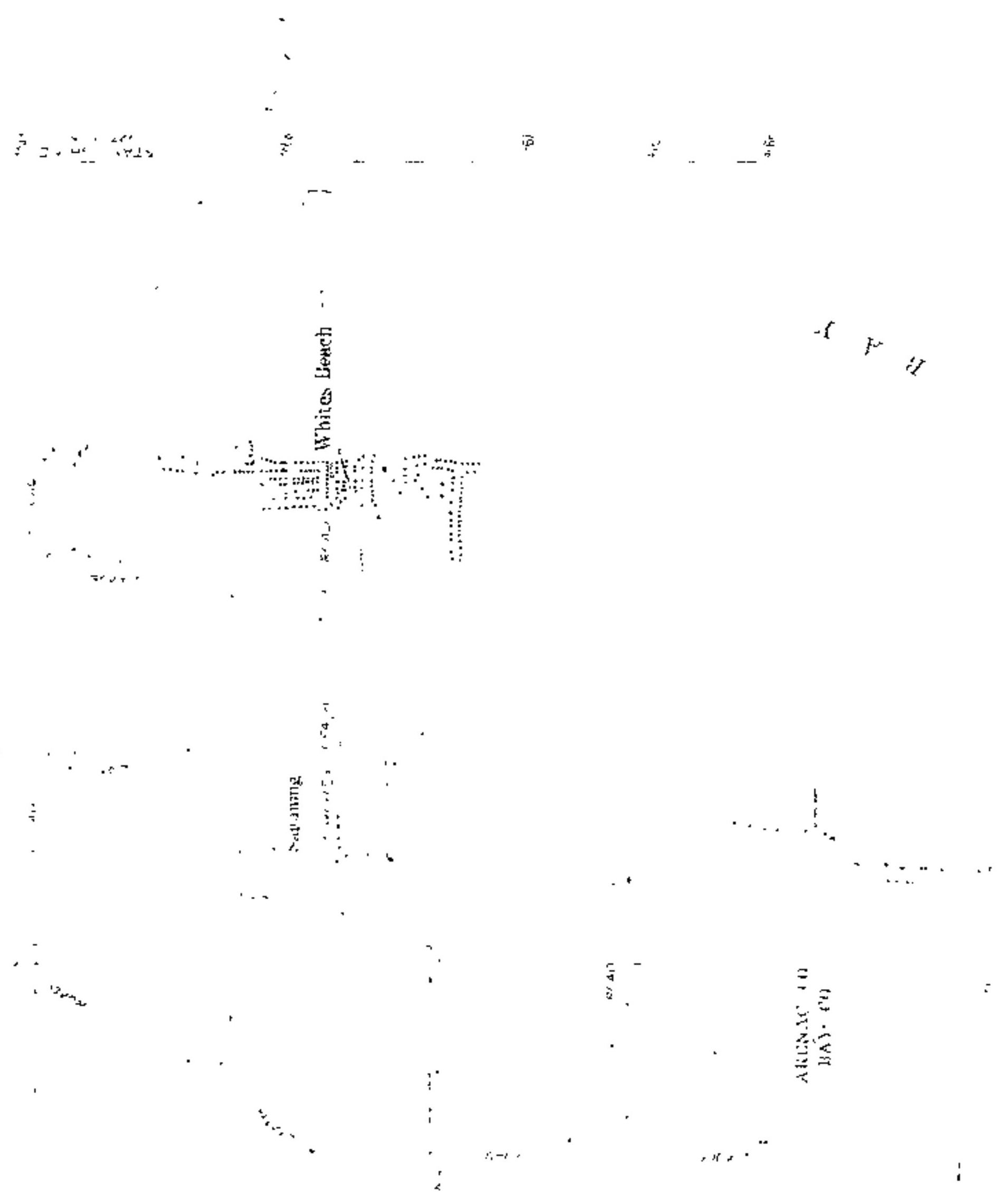


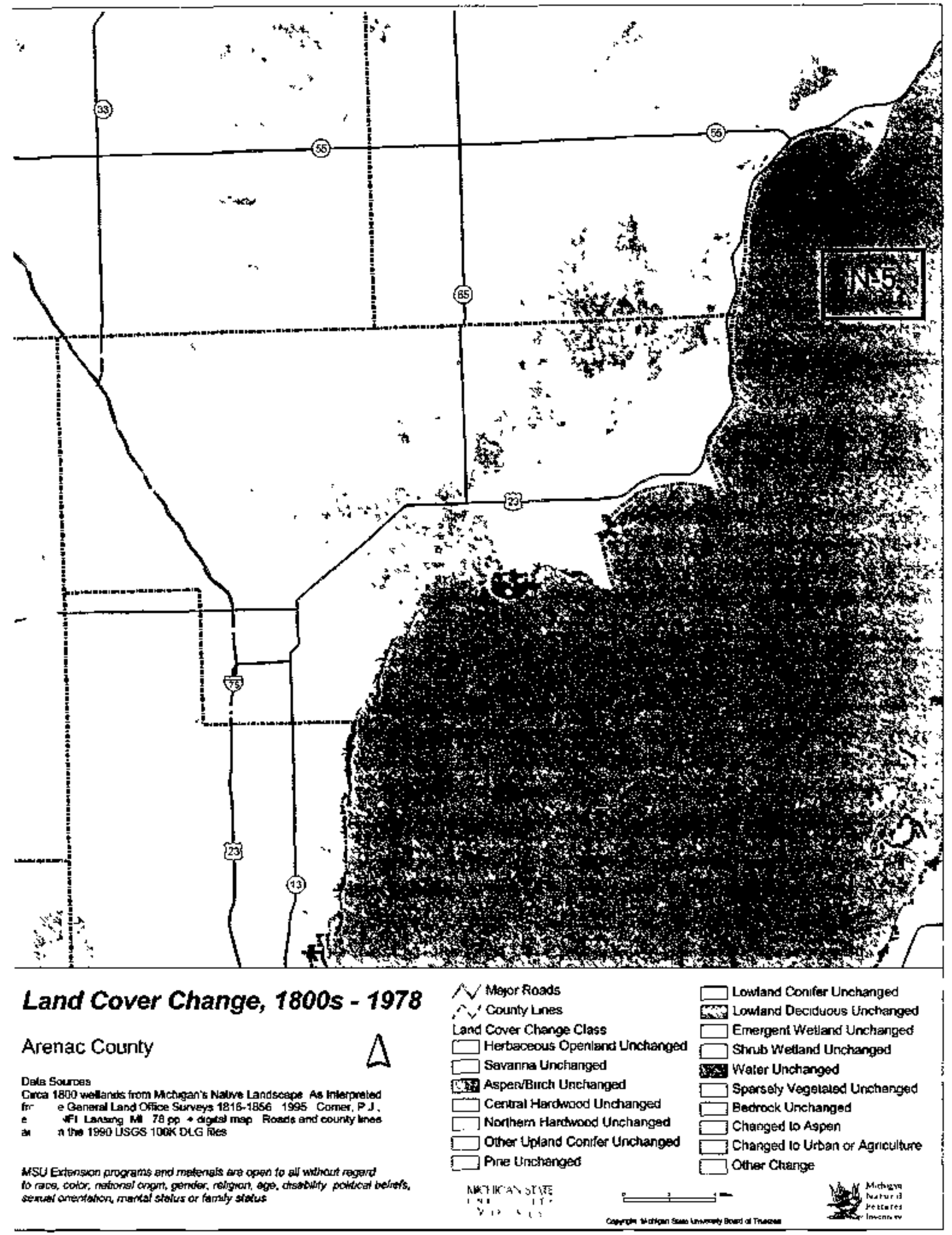




\section{N-6}

\section{Saganing River Washing Rock}

Thes tree on the Sagäneng Rivet initst ot lihe RR iracks and North of Worls Rd ragks the gerserat ares or the wasking

rorl

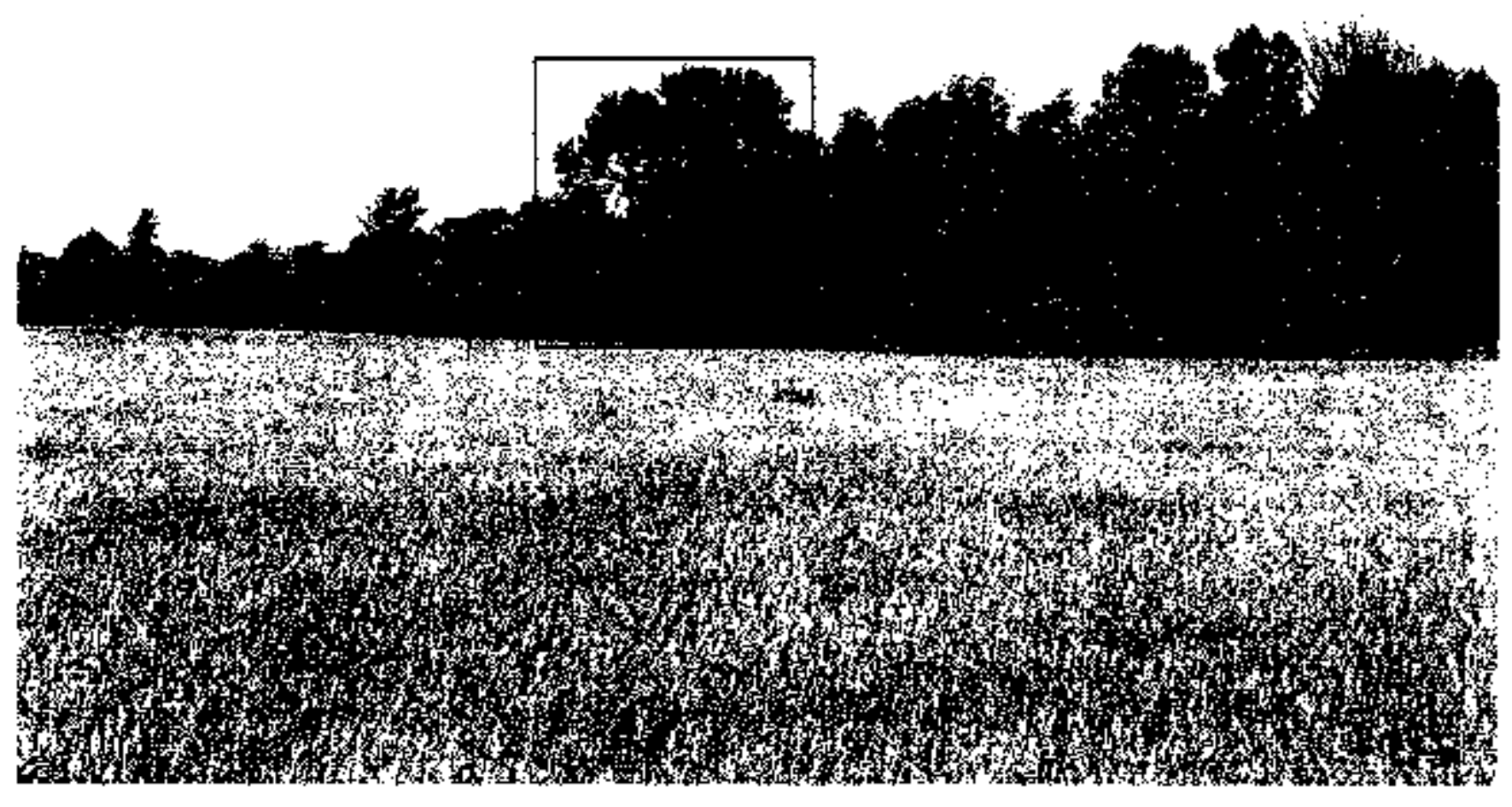

View from east side

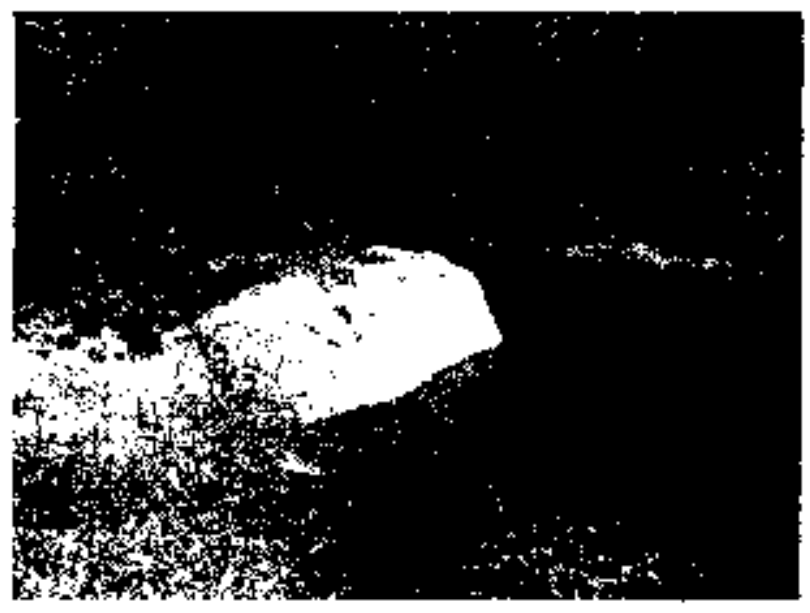

View from west side

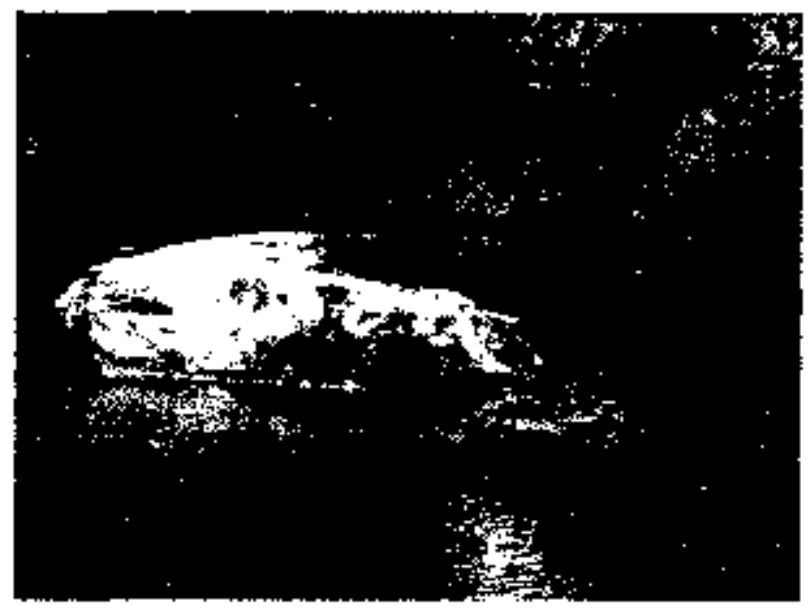




\section{Noffsinger Site plant sample}

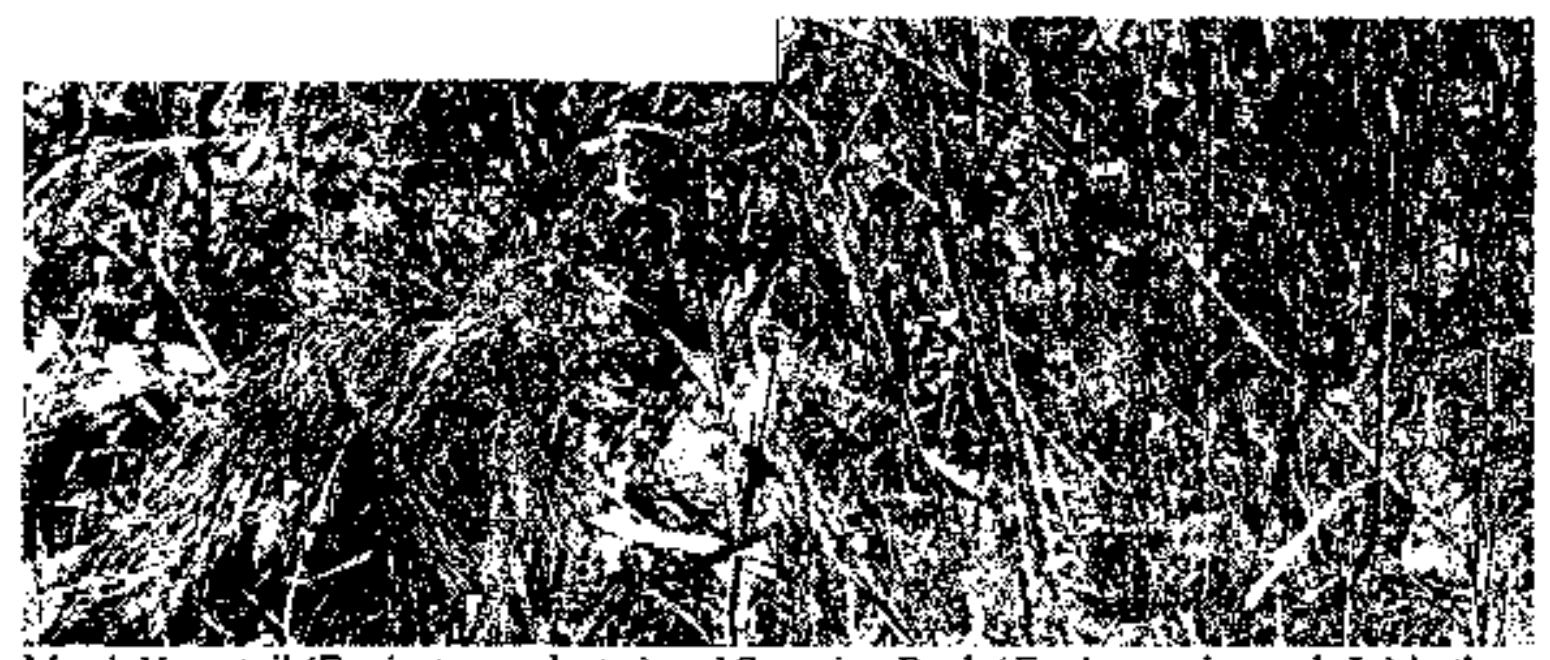

Marsh Horsetail (Equisetum palustre) and Scouting Rush (Equiserum hyemale L.) both useful in treating urinary iltness and use as an abrasive

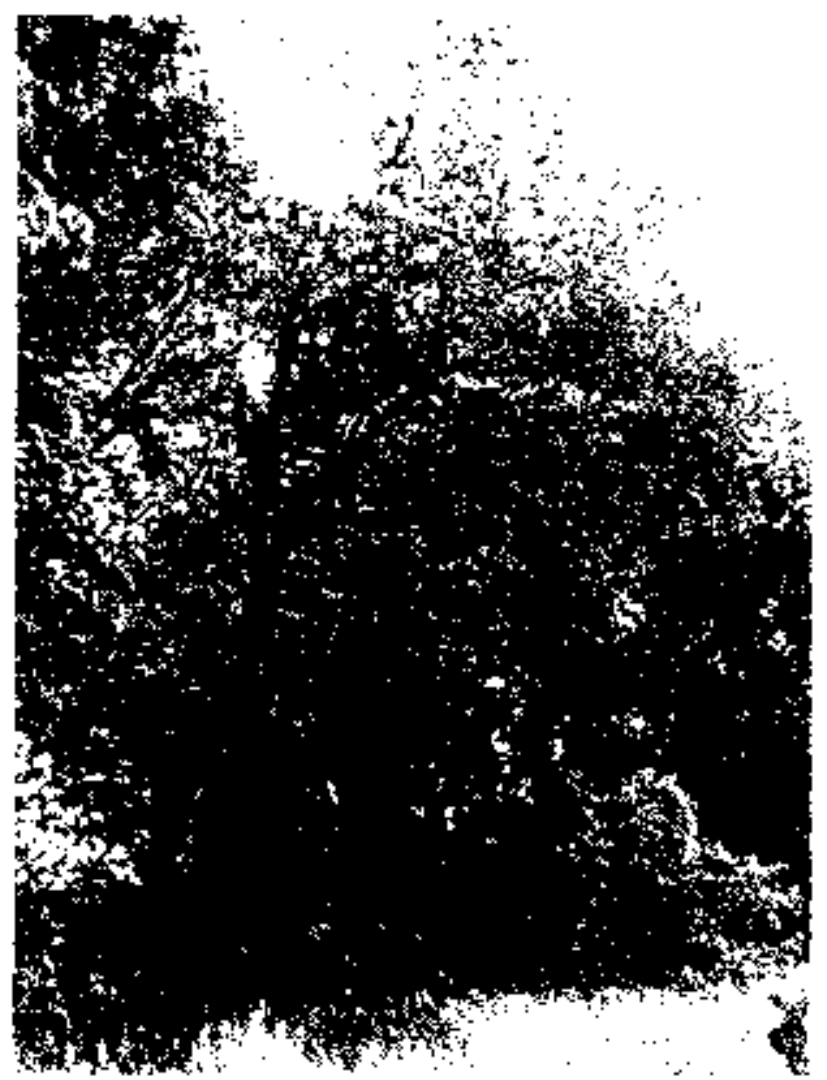

Choke Cherry (Prumts virginiono L.) and Pin Chery (Prums pensylvanica L. f. var. pensylvanica) both useful as a cough and cold remedy and a food resource 


\section{$\mathrm{N}-7$}

Elm tree either slippery elm (Ulmus rubra Muhl.) or American Elm (Ulmus americana L.) the former used for sore throat and soothing mucus membranes and both barks used for cover of lodges, containers and rattles

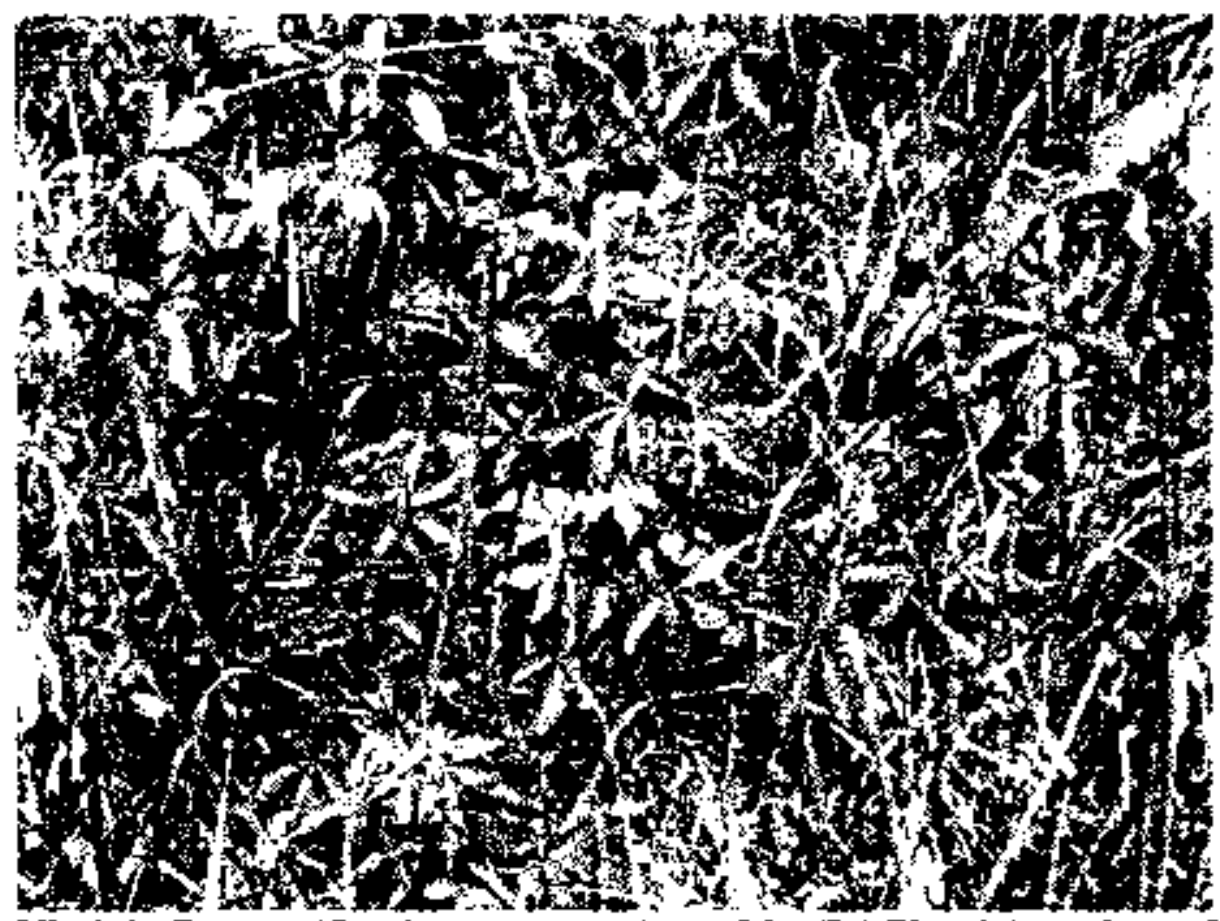

Virginia Creeper (Parthenocissus quinquefolia (L.) Planch.) used as a food and dye source, as well as a politice used for swellings

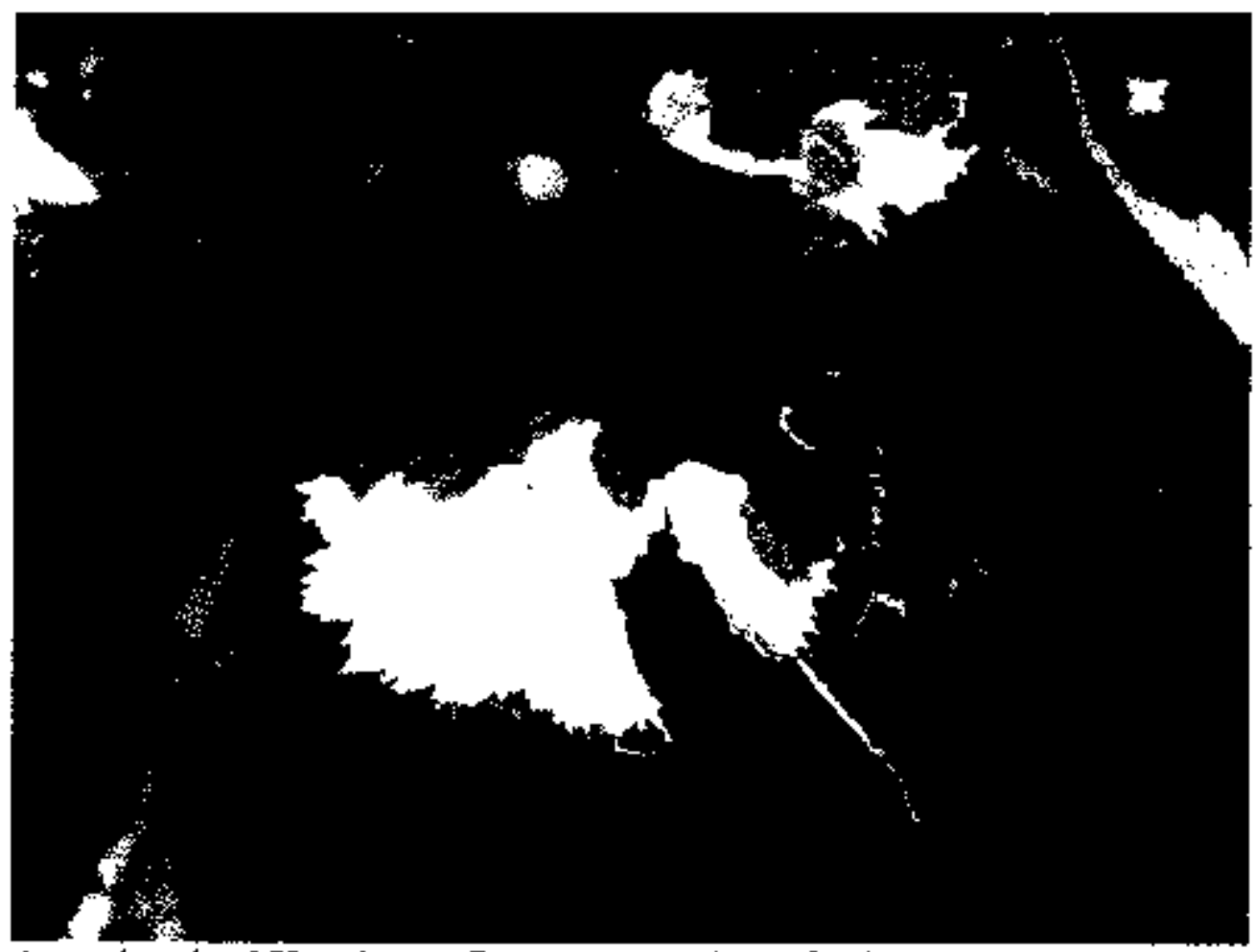

An undermined Hawthom (Crataegus) used as a food source 


\section{$\mathrm{N}-7$}

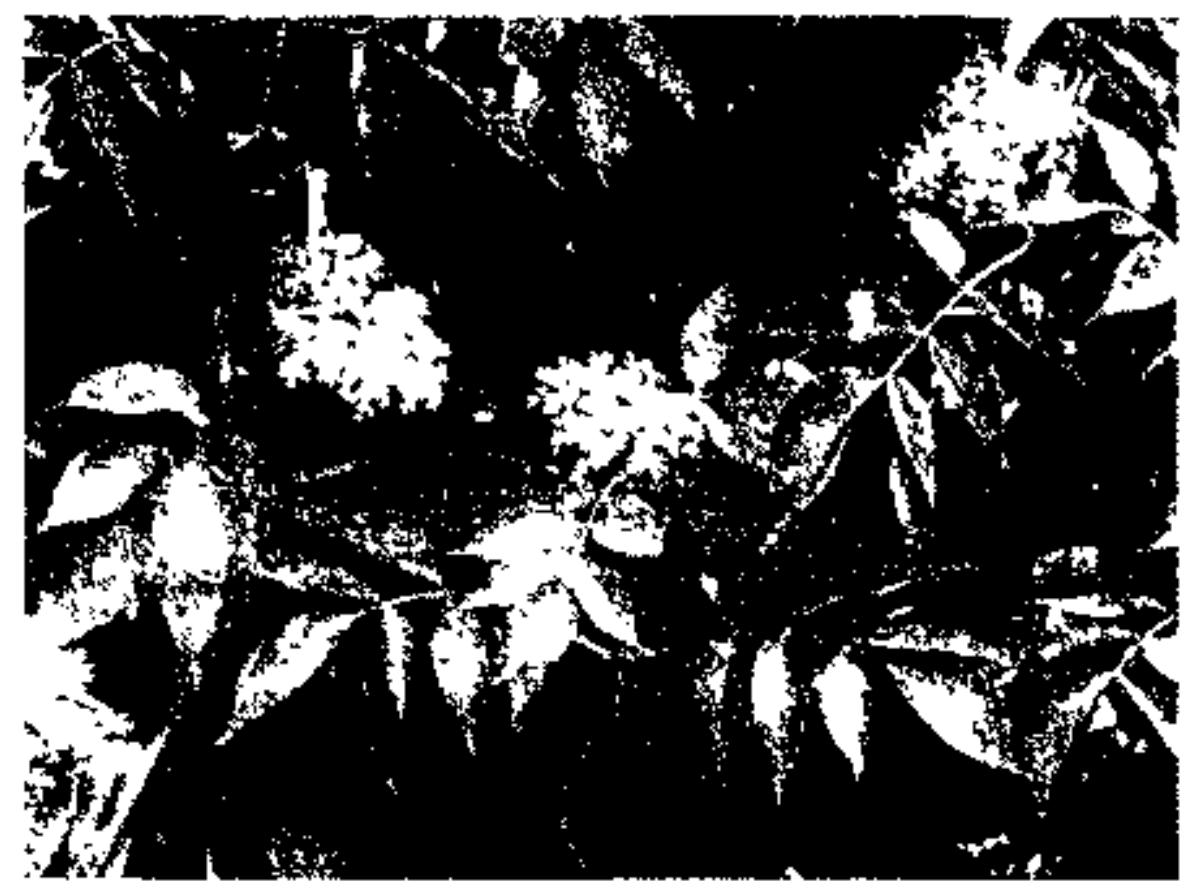

Common Eiderberry (Sambucus nigra L. ssp. canodensis (L.) R. Bolli), berty used as food, making toy flutes and as a medication to produce vomiting

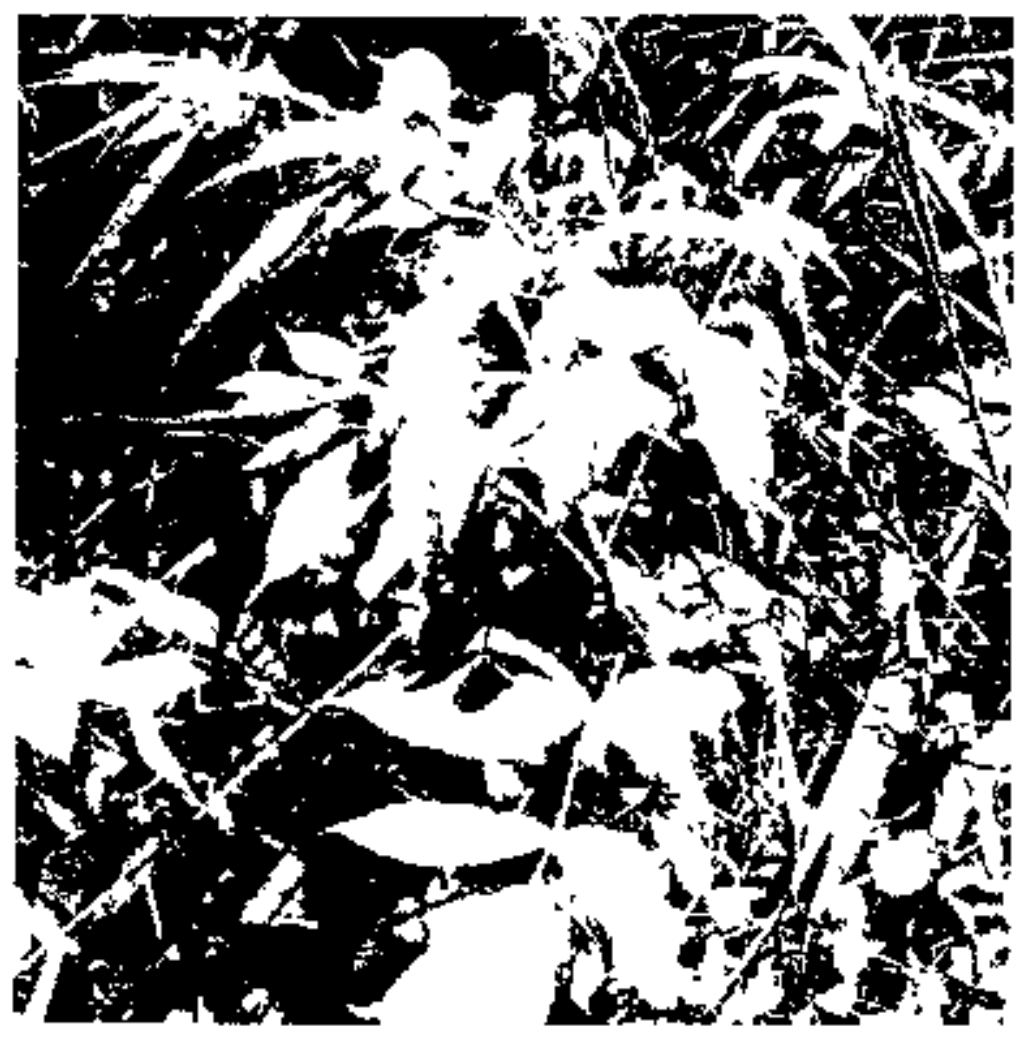

Raspberry (Rubus tdaeus L. ssp. Strigosus (Michx.) Focke) berry used as food, leaf used to promote health during pregnancy and root used as intestinat medicine 


\section{N-7}

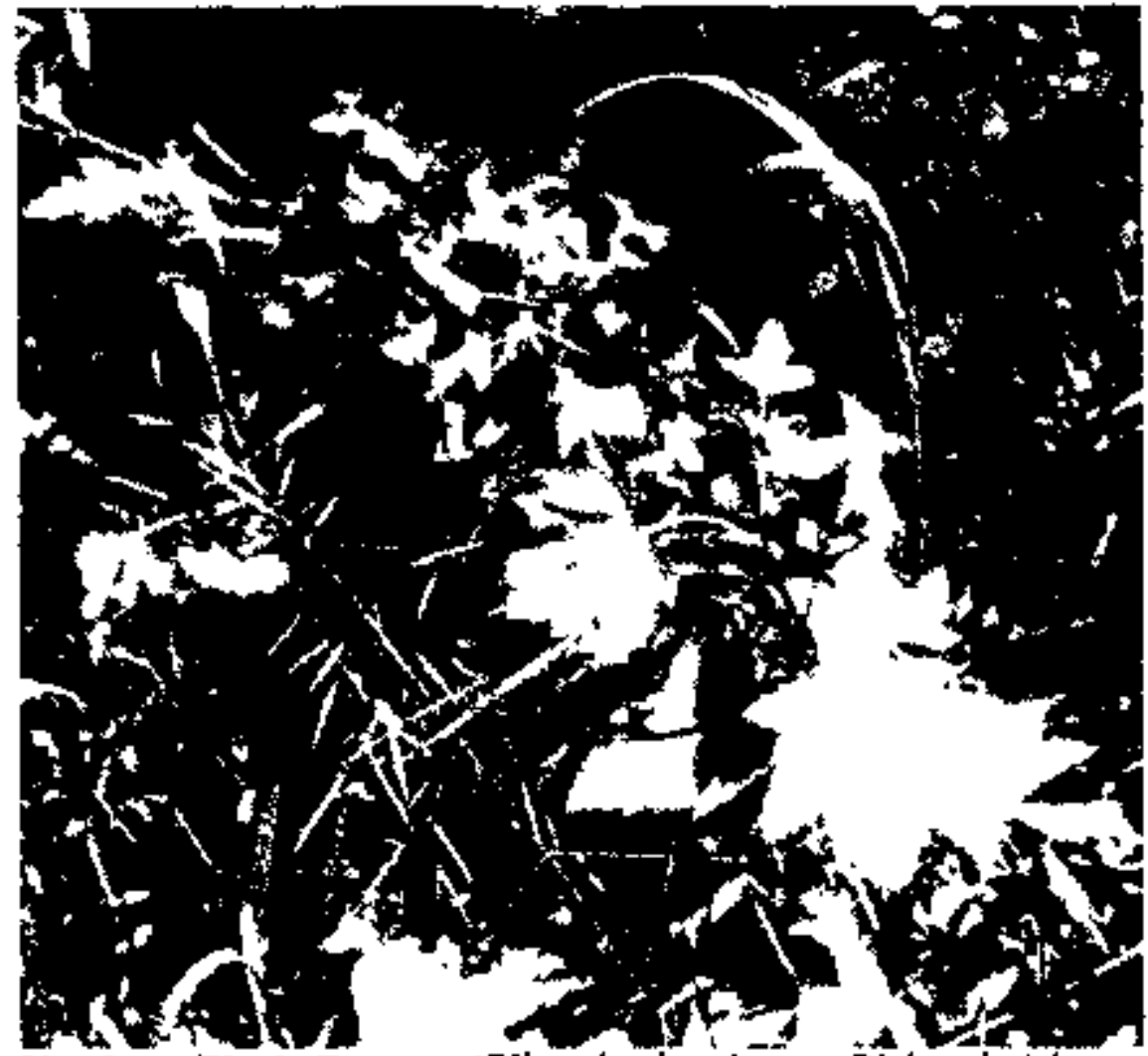

Northern Black Currant (Ribes hudsonionum Richards.) berry food source

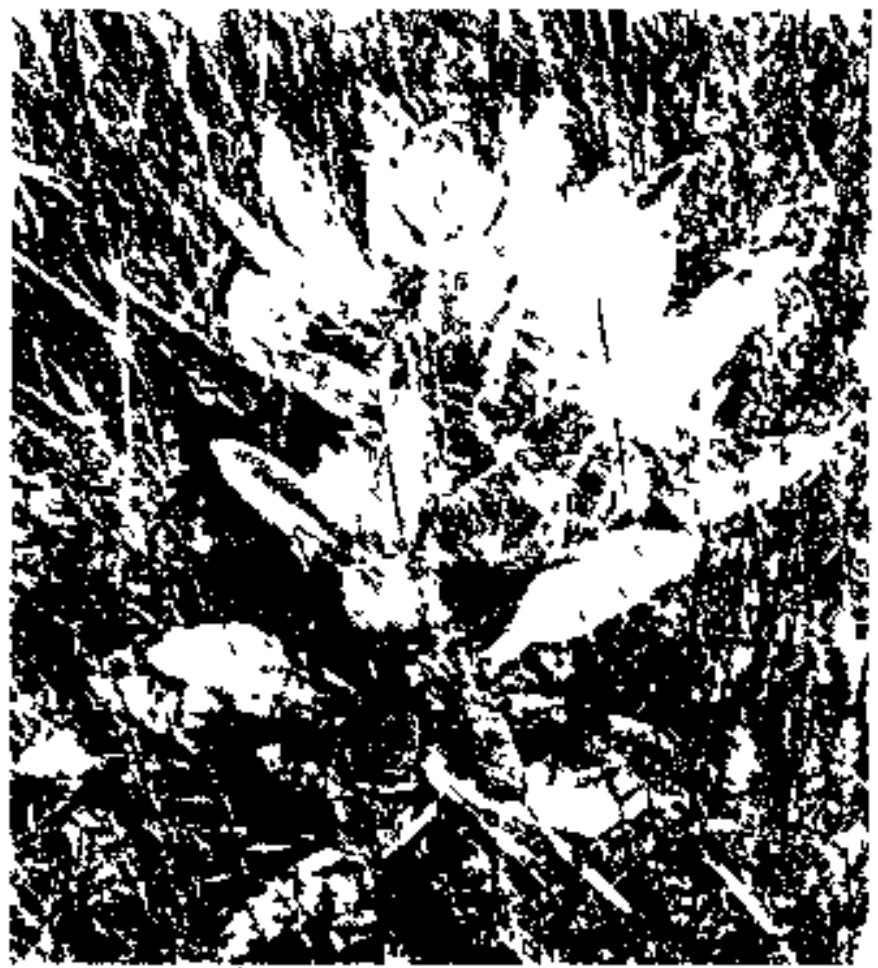

Mullein (Verbascum thapsus L,) leaf used for respiratory illness and root used as a stimulant 


\section{$\mathrm{N}-7$}

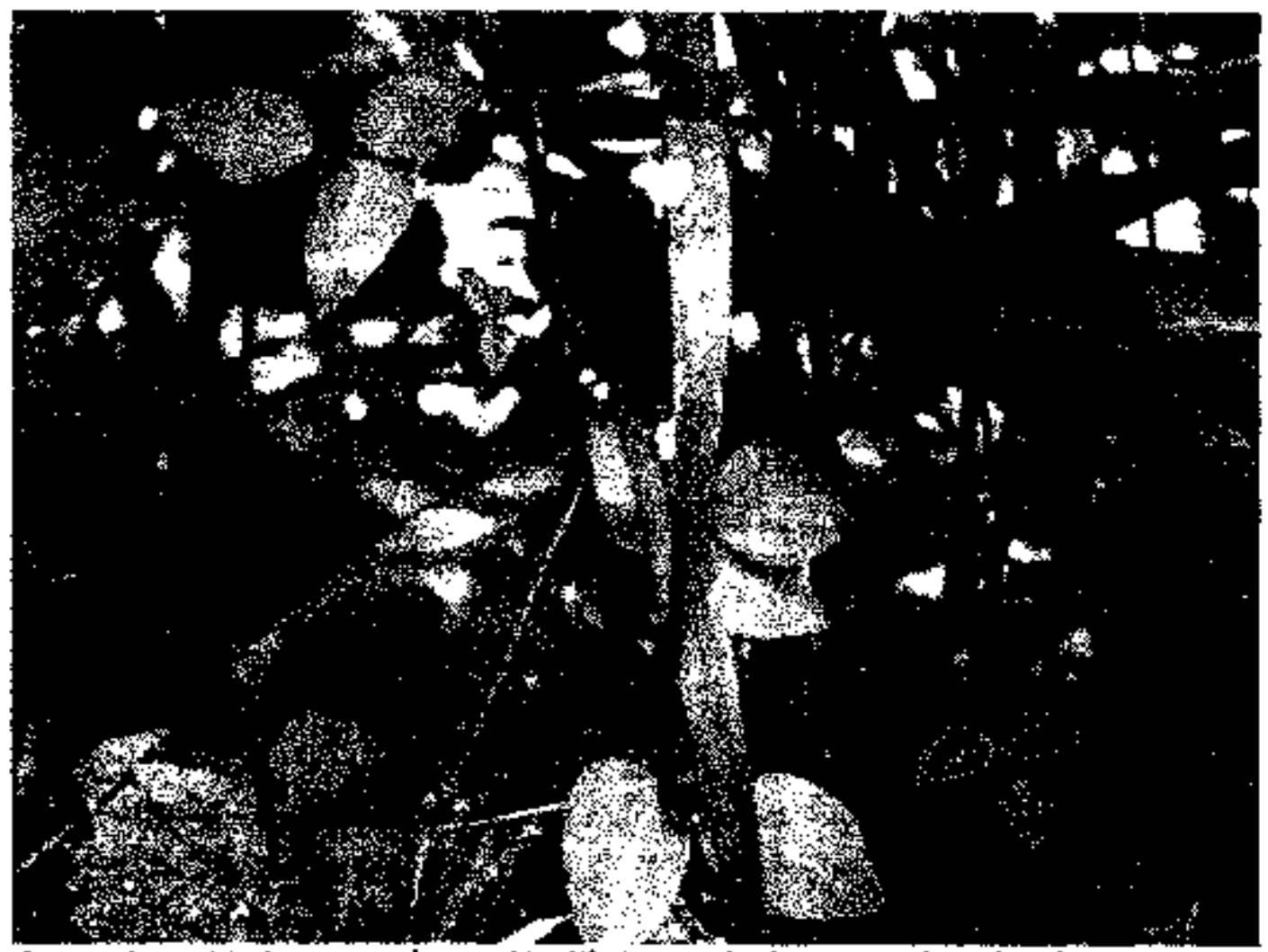

Groundnut (Apios americana Medik.) root/tubers used as food

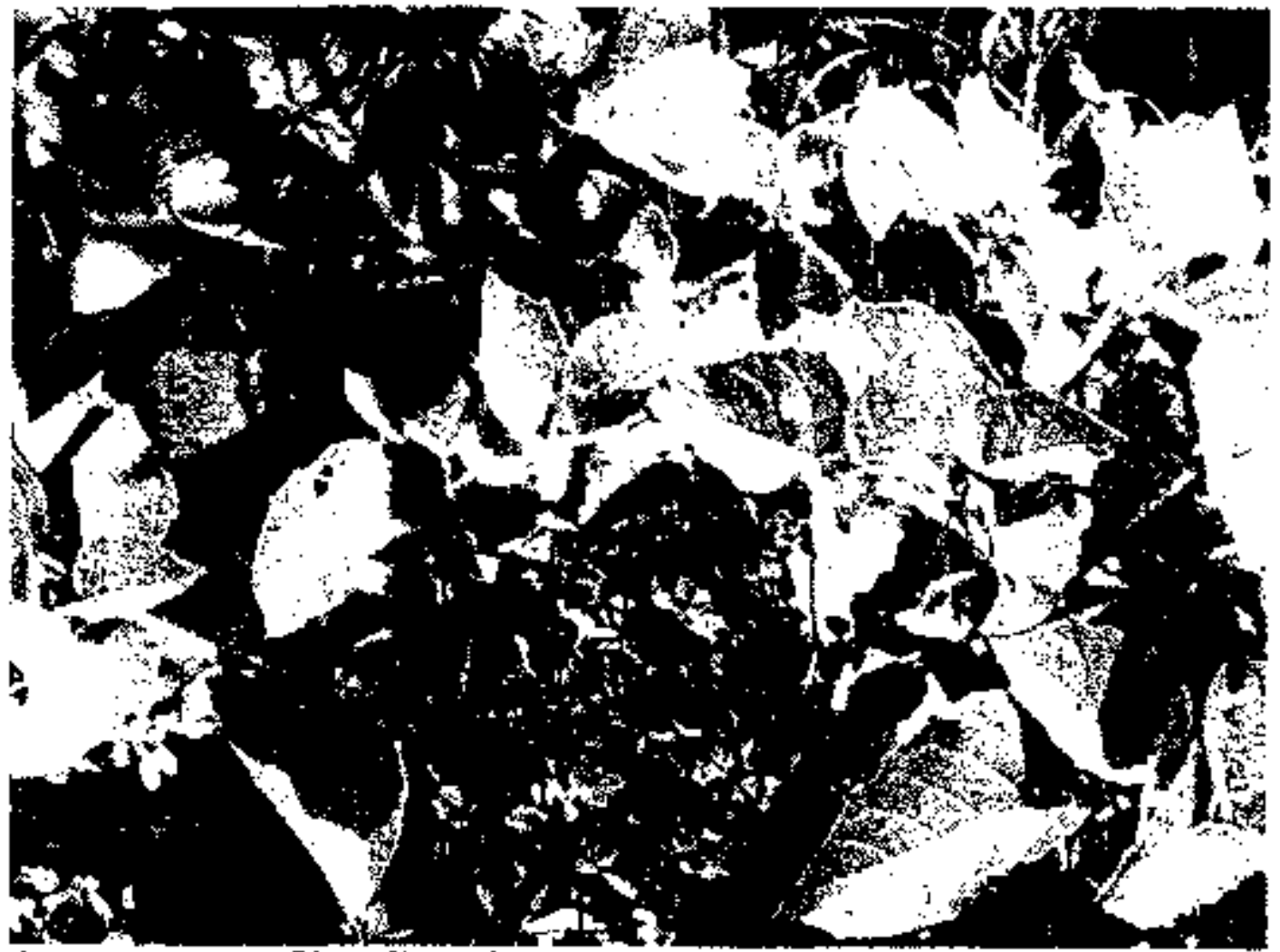

Ground Cherry (Physolis walteri Nutt.) fruit used as food source 


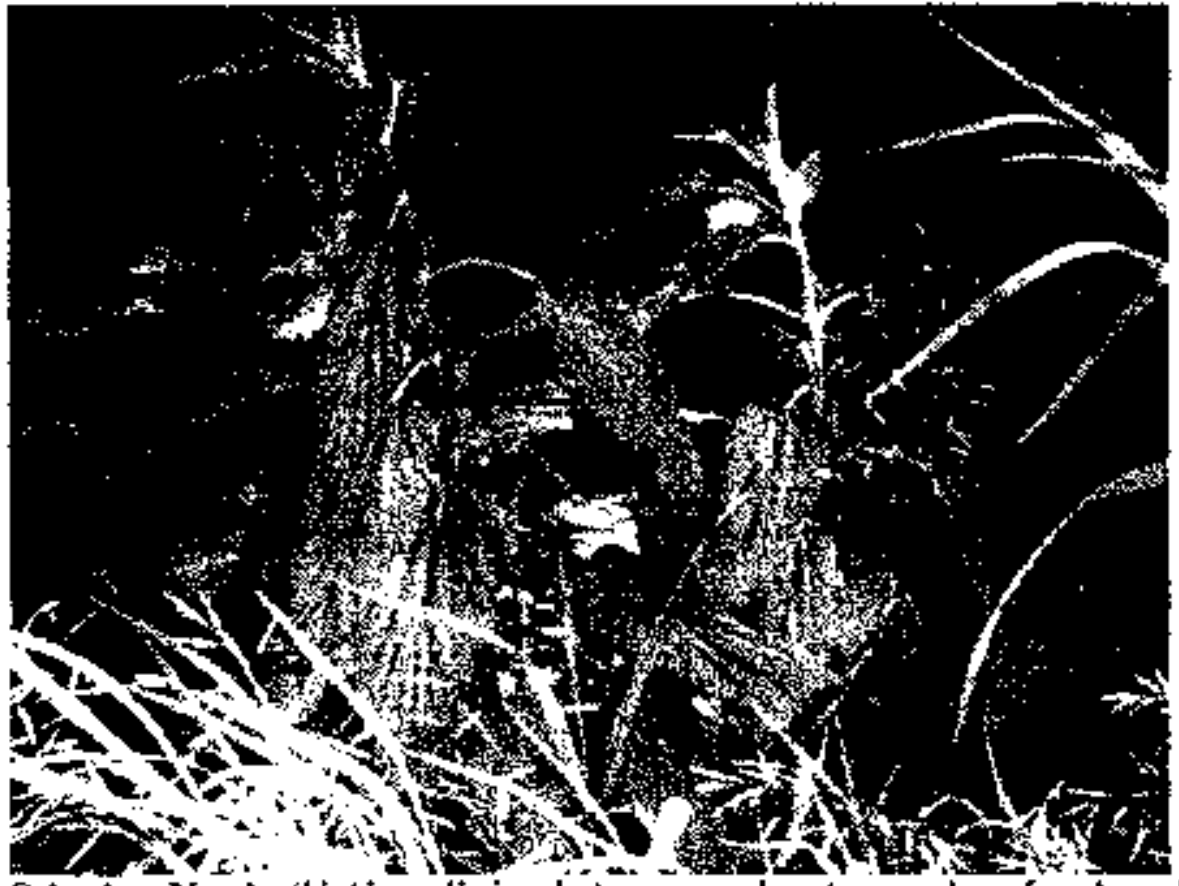

Stinging Nettle (Urtica dioica L.) young shoots used as food and stem used for fiber in fish nets, sewing and cordage

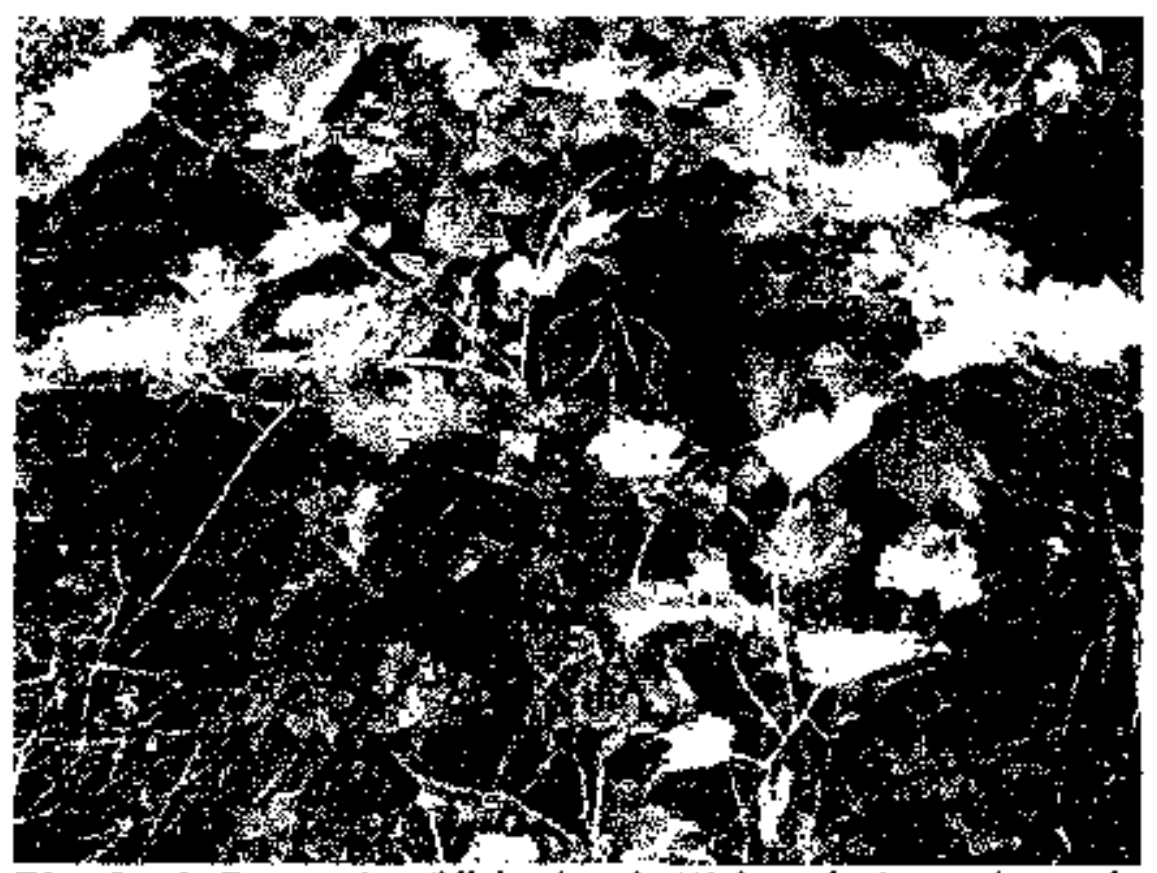

Riverbank Grape vine (Vitis riporia Michx.) fruit used as a food source, vine used as cordage and root used for rheumatism 


\section{N-7}

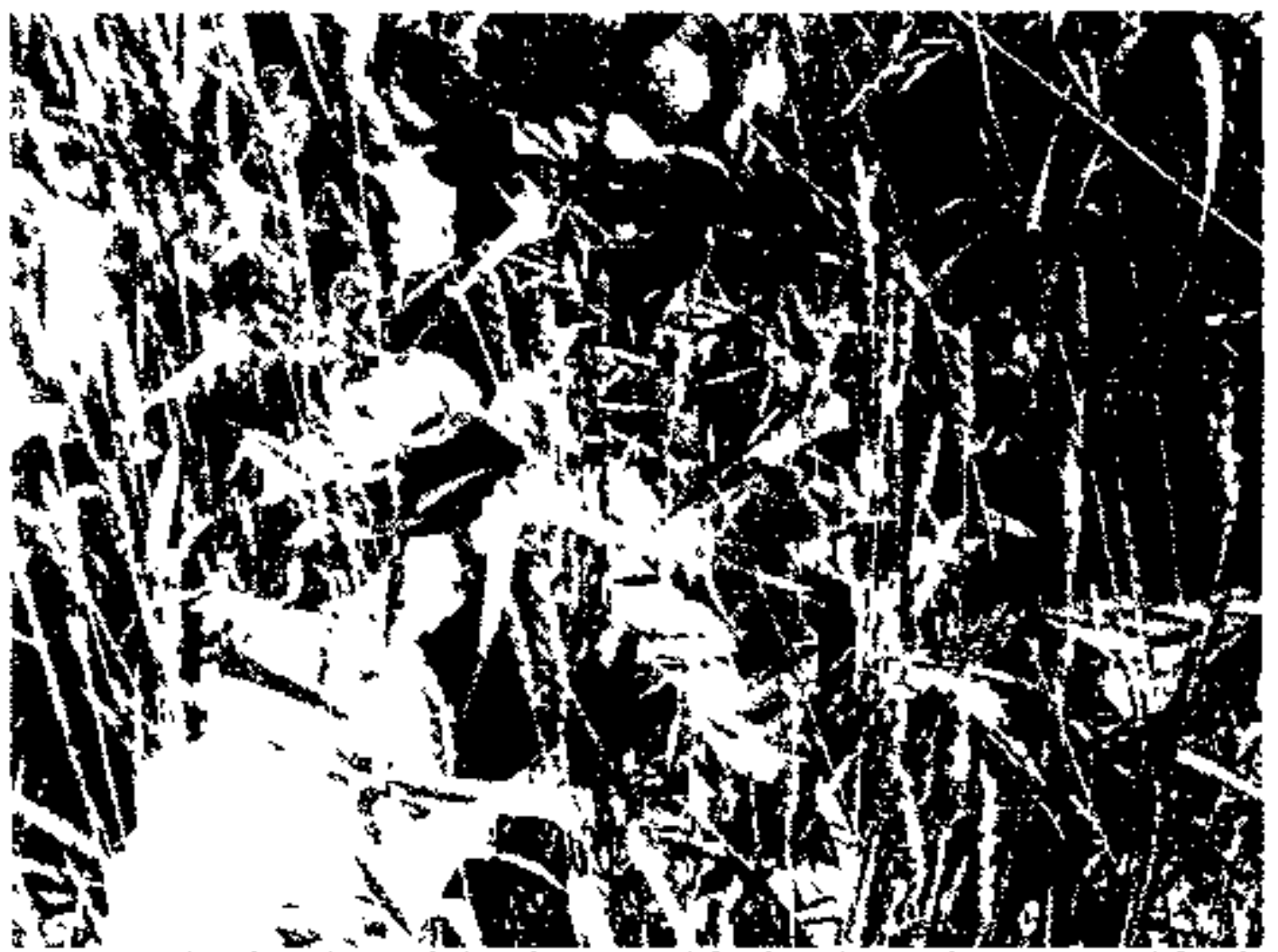

Burdock (Arctium loppa L.) poltıce used for brusies and sprains and food source

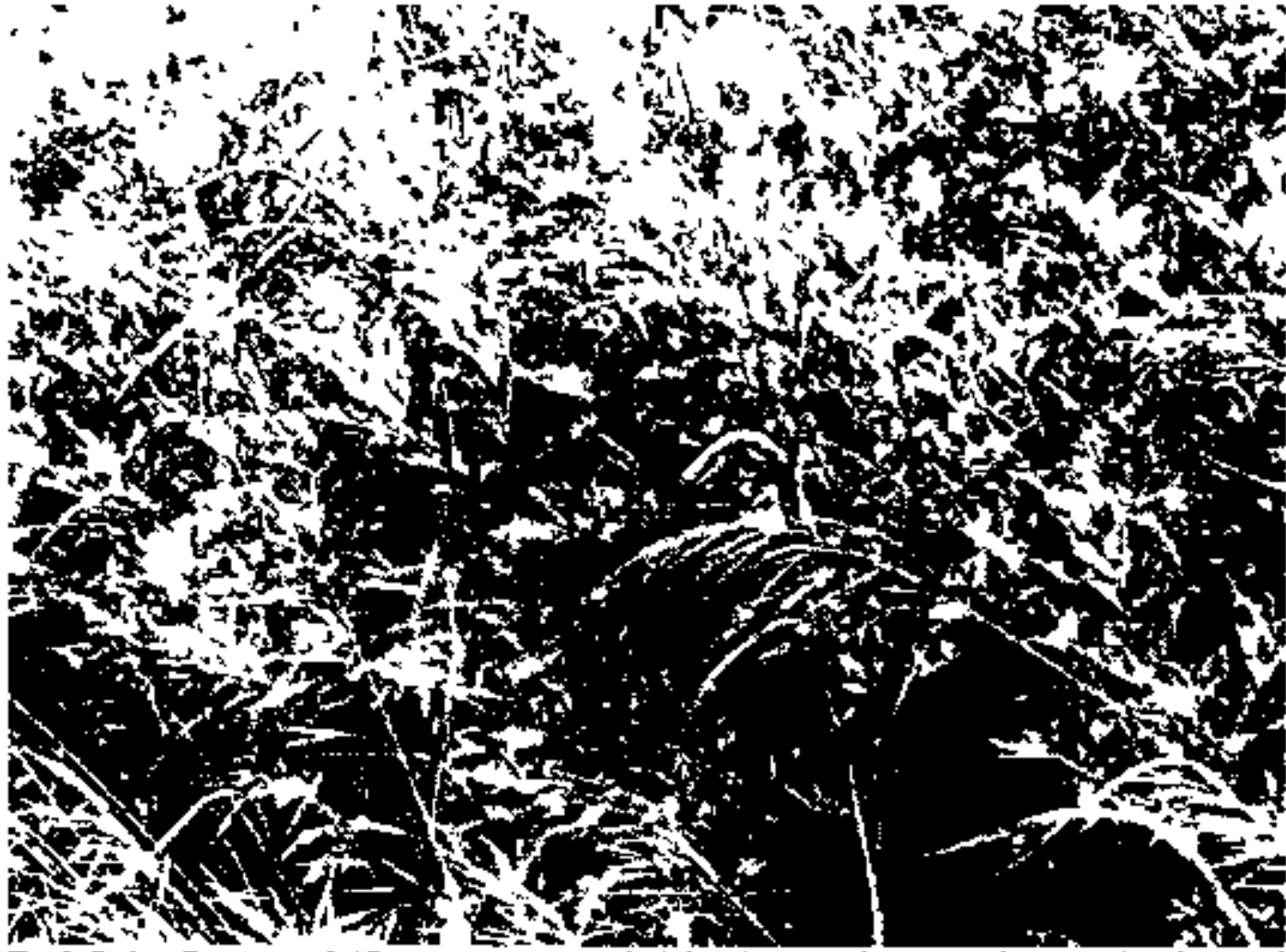

Red Osier Dogwood (Cornus sericea L.) basket making and inner bark smoked in ceremony 


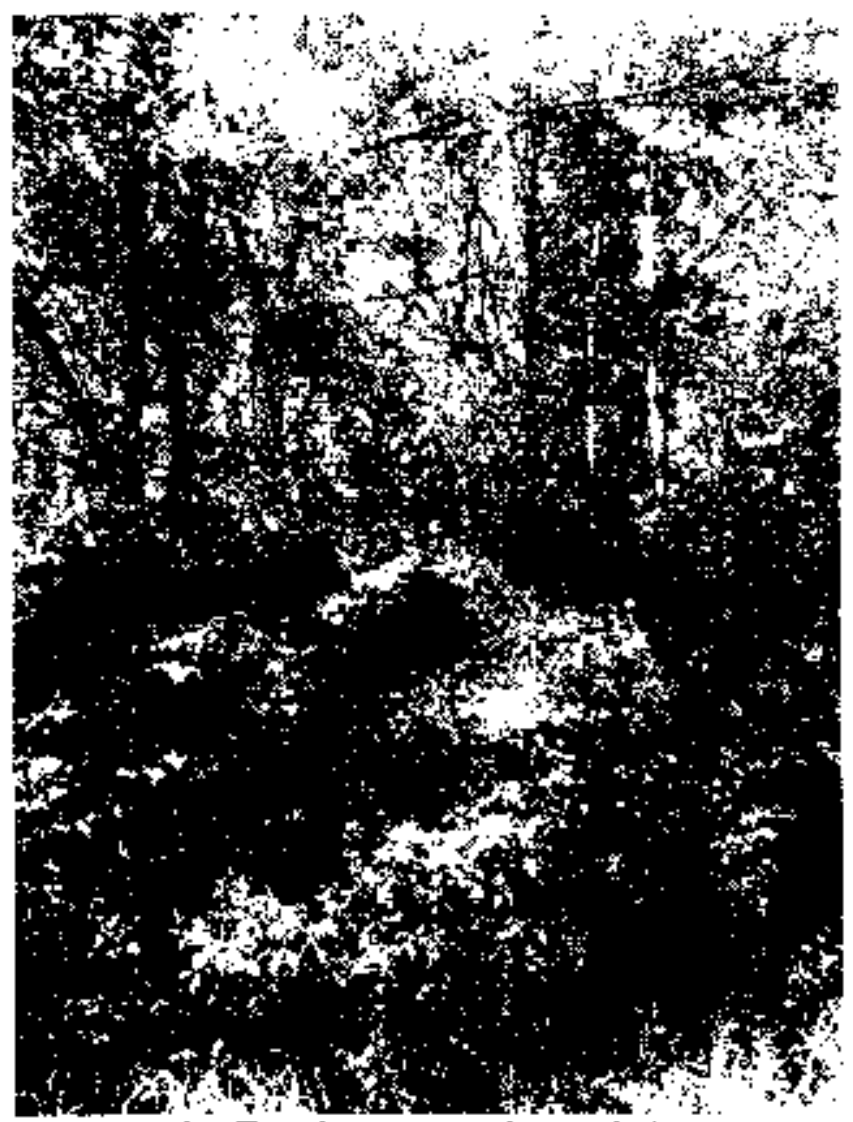

White Ash (Fraxinus americono L.) canoe and tool building material

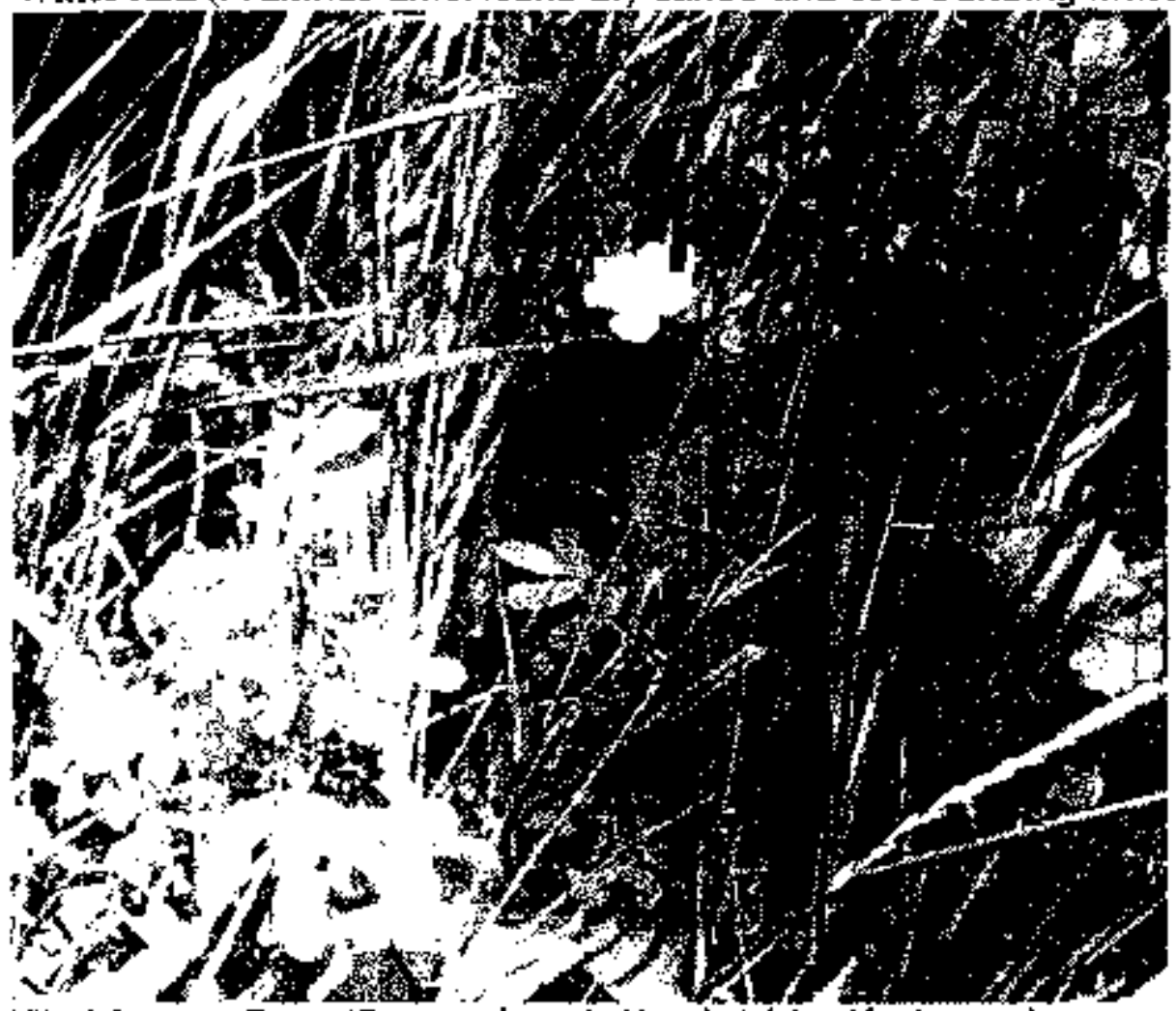

Wild Swamp Rose (Rosa palustris Marsh.) hips/fruit used as source of vitamin C 
Appendix L. Transmission Study 


\title{
TRANSMISSION ASSESSMENT
}

\author{
for \\ THE SAGINAW CHIPPEWA TRIBE OF MICHIGAN
}

submitted on

21 February, 2006

by

Distributed Generation Systems, Inc. (Disgen) Author: Krista Jo Gordon, Manager of Engineering 


\section{Table of Contents}

Transmission Assessment

Introduction

Transmission System

Interconnection Procedure

Markets

Conclusion

Appendix I - Interconnection Application Form 


\section{Introduction}

In May, 2004 Distributed Generation Systems, Inc. (Disgen) signed an Energy Services Contract with the Saginaw Chippewa Tribe of Michigan (Tribe) to explore and develop the Tribe's renewable energy resources. Shortly after, Disgen applied to the Department of Energy (DoE) for funding for the development of its wind and solar renewable resources. The total amount of the grant was $\$ 222,188$. Two meteorological ("met") towers were installed at two sites on reservation lands in December, 2004. At the time of writing, 14 months of wind data had been collected. This Transmission Assessment will discuss the interconnection options available to the Tribe for wind projects in both locations.

The first met tower is located approximately three miles east of the Town of Mount Pleasant, Michigan in Isabella County. It is near the intersection of Shepherd Road and Remus Road as shown below in Figure 1. It is on a site selected by a professional meteorologist as having potential for a wind project. The wind turbine(s) will be located in close proximity to the met tower.

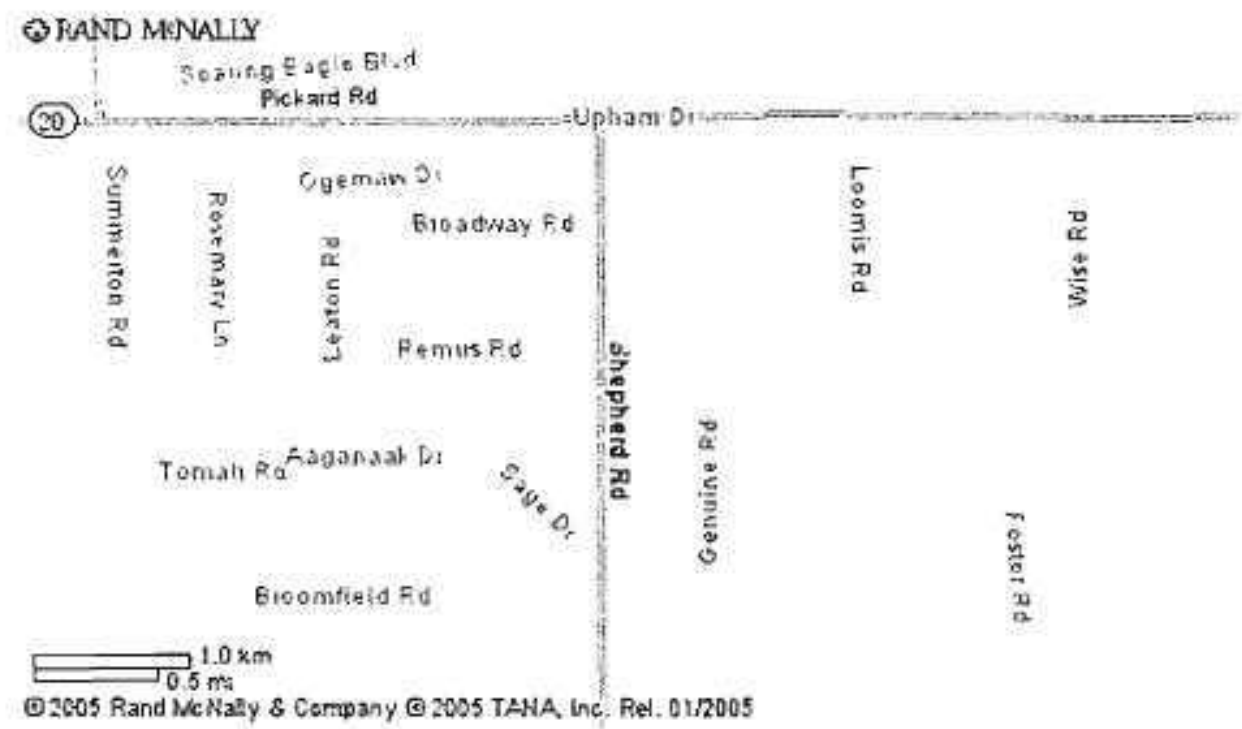

Figure 1, "Mount Pleasant Road Map"

The second met tower is located approximately two miles west of Whites Beach in Arenac County, Michigan. It is near the intersection of Worth Road and State Road as shown below in Figure 2. The closest town larger than Whites Beach is Standish, MI. This site was also selected by a professional meteorologist. This met tower is also close to where the wind turbines will eventually be situated. 


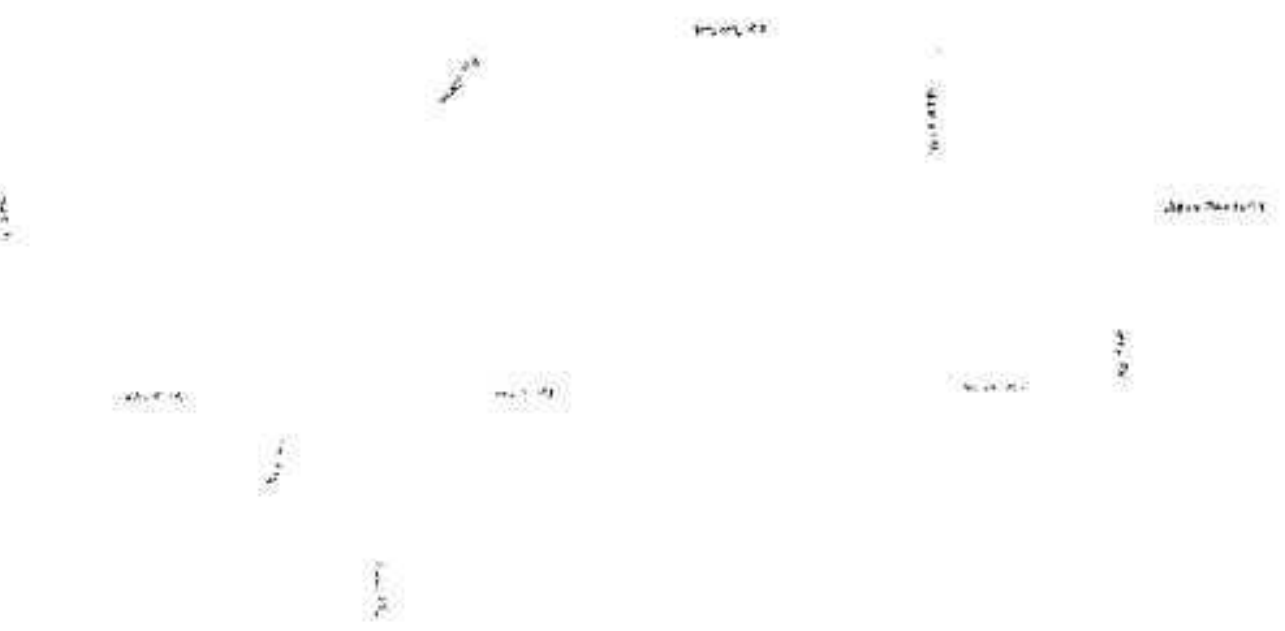

Figure 2, "Standish Road Map"

Both sites have a small amount of developable reservation land, so accordingly the wind projects will be small. This report will determine the maximum amount of wind turbines that can reasonably be interconnected in both locations. If it is determined later that there is less land than can accommodate the maximum project size with regards to interconnection, then the project sizes will probably have to be decreased.

The nature of the wind resources at the two sites will not be addressed in this Assessment since Disgen was contracted to perform this Transmission Assessment regardless of the economic viability of the wind power projects.

\section{Transmission System}

The Tribe has not designated minimum, maximum, or target project sizes. This report will explore the feasibility of interconnecting small projects in both areas and will attempt to offer insight as to the most appropriate size of project that can be interconnected to the local electric power system without requiring extensive upgrades and expansions. Both thermal limits and voltage drops will be considered. There are many factors that determine the feasibility of interconnecting a new generator to an existing transmission system. Chief among these are equipment voltages, size ratings, other regional loads and generators, and market. All of these will be discussed herein. However, it is worth noting that this report is not an official interconnection study since it has not been written or reviewed by any transmission agency, although it was written with input and interviews with relevant regional utilities.

\section{Standish}

From Disgen's telephone interviews with Consumers Energy ("Consumers") Disgen has learned that the Duquite Substation is the closest substation to the met tower location. It is located just northwest of the intersection of Palmer Road and Foco Road, approximately three miles north of the met tower. The street address for the Duquite 
Substation is 3755 East City Limits Road, Standish, MI. The Duquite Substation has buswork at $138 \mathrm{kV}, 46 \mathrm{kV}$, and $24.9 \mathrm{kV}$.

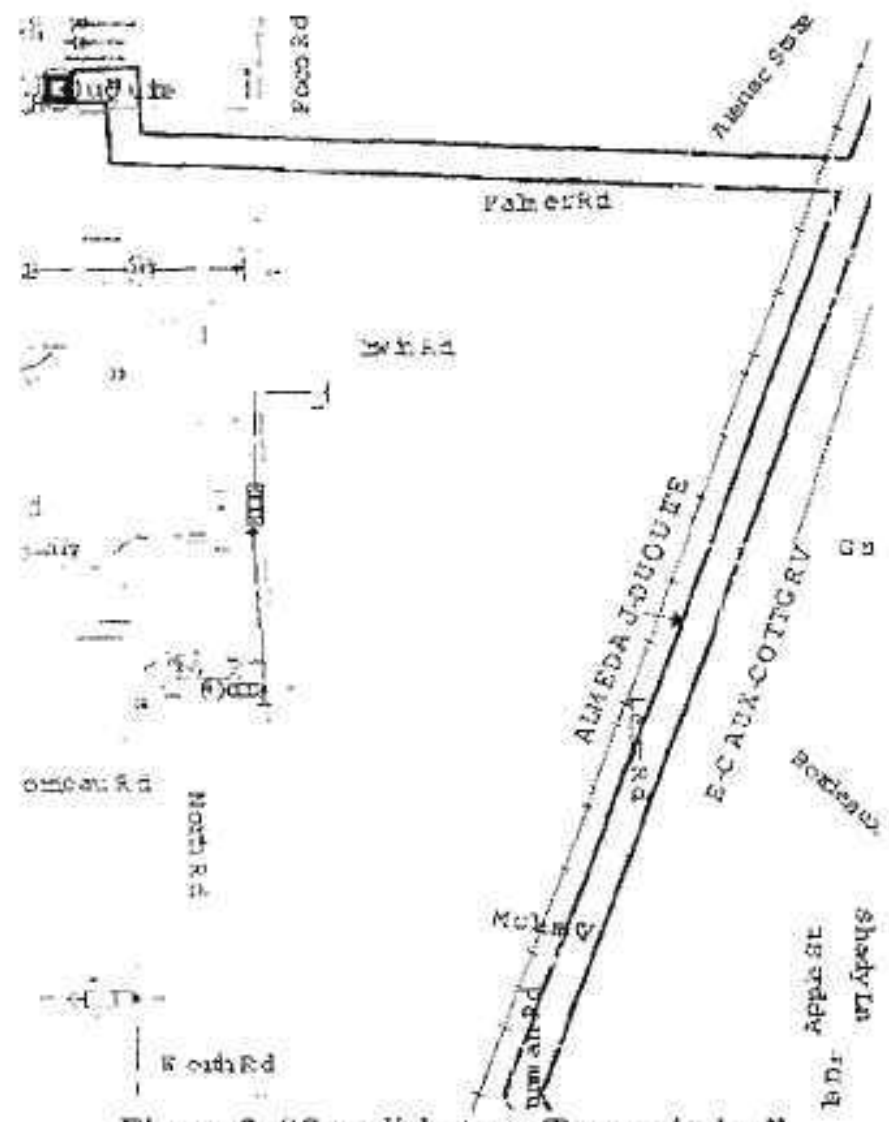

Figure 3, "Standish Area Transmission"

The second and third options for interconnection at the Standish site are two $138 \mathrm{kV}$ transmission lines that pass less than one mile from the met tower. One connects the Carn Generating Station with Cottage Grove and East Tawas. The other connects Saginaw River, Duquite, and Twining. For either of these two interconnections, the formal request for study work would go to the Michigan Electric Transmission Company - NO W TC (METC). For both of these two options, the size of the wind project near Standish would be far less than the thermal limit of the lines since there is not sufficient land to support a project that would appreciably impact these lines. However, similarly there may not be enough land to build a project large enough to absorb the cost of interconnecting at a high voltage.

Also in close proximity to the project site is a two-phase distribution line running about four miles along Worth Road. It would have to be changed to three-phase in order to connect to the $24.9 \mathrm{kV}$ bus at Duquite. It is estimated that this line could be rebuilt for less than $\$ 100,000$ per mile.

Voltage drop is another consideration for the project. It is a function of conductor size, length, and current flow. Since the distance from the project to the interconnection is 
relatively short ( $3-4$ miles) and since the project would build this feeder to its own specifications, the voltage drop can be minimized in the feeder's design. It is not expected that the full-load voltage drop will exceed $2-3 \%$, and this is well within a range considered reasonable by most utilities.

Based on market considerations discussed below and the state of the existing electrical infrastructure near Standish, Disgen recommends interconnection to either the $24.9 \mathrm{kV}$ bus or the $46 \mathrm{kV}$ bus at the Duquite Substation. In either case, the project will build a small step-up substation on the project area to increase the voltage from that of the wind turbine generator(s) to either $24.9 \mathrm{kV}$ or $46 \mathrm{kV}$. From there a $3-4$ mile feeder will be necessary as well as a new recloser, meter, and telemetry equipment at the Duquite Substation. The size of the project would be capped at approximately 10MW.

\section{Mount Pleasant}

For the Mount Pleasant site, there is no high voltage transmission nearby. Instead, the two options are the Tribe's Casino or a $46 \mathrm{kV}$ distribution line nearby. They are roughly equidistant from the project area. If a connection at the Casino is made, then it should be "behind the meter". In other words, the wind project should connect to the Casino side of the meter rather than the grid side. This way, the output of the wind project can directly serve the load at the Casino and offset its retail electricity rate. The cap for net metering on the Consumers Energy system is $30 \mathrm{~kW}$, so any excess beyond $30 \mathrm{~kW}$ more than the Casino's spot load would be sold back to Consumers at a specially negotiated rate. The other option is to connect directly to the $46 \mathrm{kV}$ distribution feeder in which case the output would be sold at Consumers' wholesale rate for wind energy. Given that this is lower than the Casino's retail rate, Disgen recommends direct connection to the Casino.

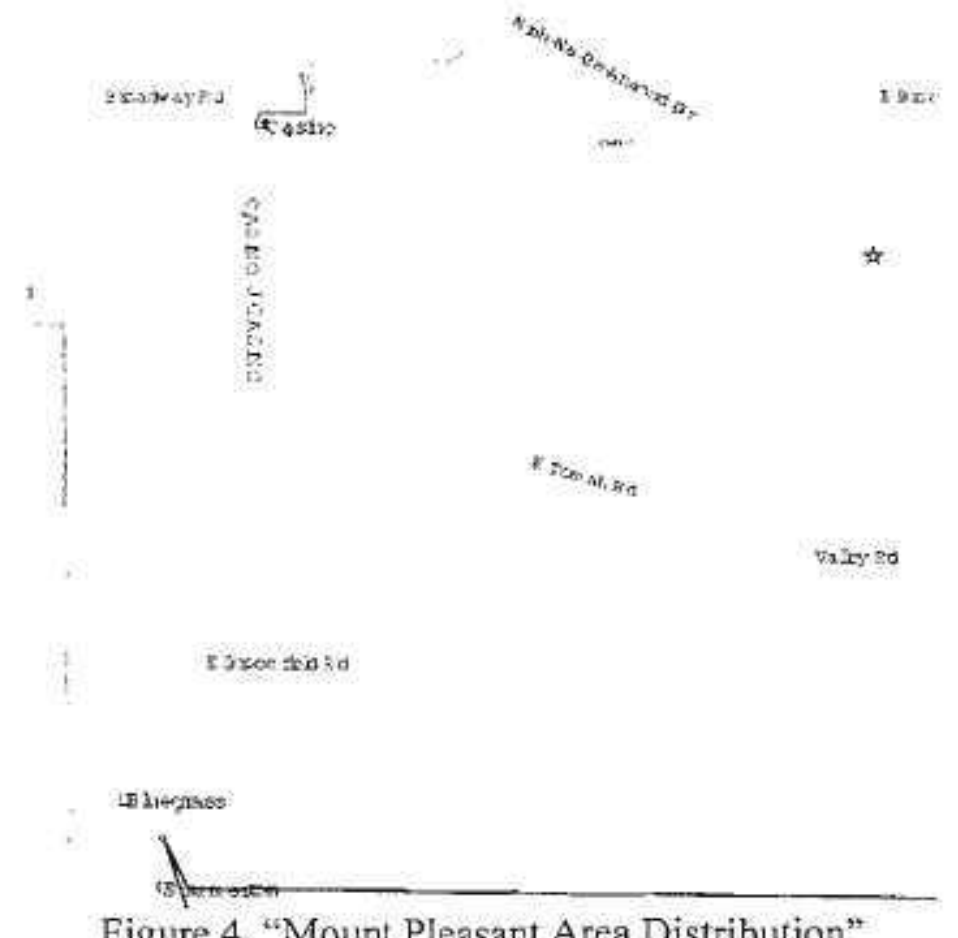

Figure 4, "Mount Pleasant Area Distribution" 
The precise size of the project is best determined after conducting a load analysis of the Casino, which is beyond the scope of this assessment. However, Disgen is well-qualified to perform such an analysis and would be pleased to offer its services to the Tribe. It is expected that the total size of the wind project will stay under approximately $5 \mathrm{MW}$.

\section{Interconnection Procedure}

Once the Tribe receives its final wind assessment report from Disgen and if it decides that it wishes to pursue a wind power project, it will be appropriate to file an Interconnection Application with Consumers Energy. A blank Interconnection Application form is included as Appendix 1. This applies as long as the total project size is at least 2MW and requires a fee of $\$ 500$ to be submitted at the same time as the Interconnection Application form. It is important to know many technical aspects of the generator(s) selected for interconnection when this form is completed. Disgen is experienced in both selecting the most appropriate wind turbines for a given wind site and providing detailed electrical information as required. Disgen would be pleased to assist the Tribe in completing both of these activities when the time comes. The same form and procedure apply to both the Mount Pleasant and Standish sites. They should not be combined into one Interconnection Application since they will be viewed by Consumers as two separate and distinct projects.

The interconnection study and agreement procedure is separate from the process to request and obtain transmission service.

The total cost for the interconnection study will not exceed $\$ 10,000$ and the time is not to exceed 18 weeks. The 18 -week period is meant to start the date a queue position is issued, which is the same as the date Consumers accepts a compliant Interconnection Application form from the customer. It is meant to end when the interconnection agreement is signed. This is a relatively brief and inexpensive interconnection process compared with that of other utilities in the US.

Part of the information required in order to start the interconnection study is the identity of the wind turbine(s) to be used for the project. The study will be specific to that wind turbine, so it is not advised to change models after the study. Otherwise the study may have to be repeated. The selection of a wind turbine for a project involves several determining factors. Among them are purchase price, electrical accessories available for purchase with the turbine, and most importantly the project-specific energy output in accordance with the wind data collected on the site and the air density calculated at the site. Disgen has close relationships with all of the major wind turbine suppliers currently selling equipment in the US today and is well-qualified to assist the Tribe in selecting the best wind turbine for the site(s) when the time comes.

The specific electrical requirements of the wind project and its interconnection point will be determined in the course of the interconnection study, but certain system-wide requirements have been published by Consumers that shall apply to all new generators seeking interconnection service. A generic "one line" diagram of a tentative 
interconnection for a wind project is shown below. Depending on the voltage of the line to which the project will connect, the Power Transformer on the right might not be necessary.

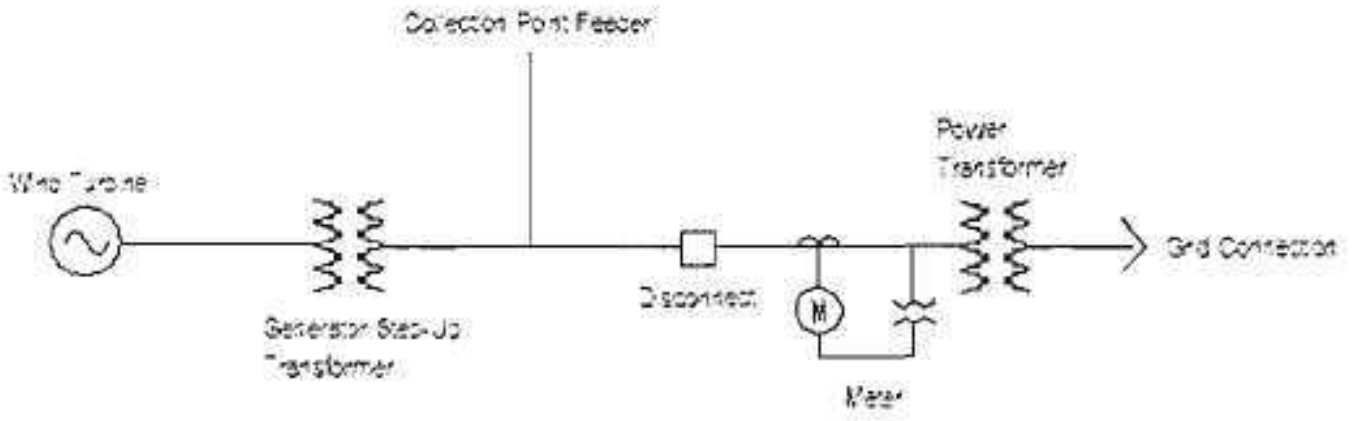

Figure 5, "Tentative One-Line Drawing"

In particular, the elements in the drawing in Figure 5 will most likely have the following capabilities and attributes as stipulated by Consumers. All equipment will be required to have adequate ratings to ensure the safe operation of the wind project and local electric power system. All equipment must also be approved by Consumers Energy.

- Transformers: Load tap changing on the low and/or high voltage side to accommodate minor fluctuations in line voltage. Also may not have a grounded-wye : ungrounded-wye connection configuration.

- Disconnect: Load break capability. Also gang operation if so designated by Consumers.

- Wind Turbine(s): Voltage regulation capability.

In addition to the equipment shown in the one line drawing in Figure 5, a relay will be necessary to allow the protective equipment at the interconnection point to operate. A current transformer and potential transformer will be necessary for the relay as shown below in Figure 6. They will be limited to 5 Amperes and 120 Volts respectively. A back-up power source for the relay will be required to ensure that it continues to operate properiy even in the event of a grid failure. The relay must be designed to prevent the wind project from re-energizing after a fault without the rest of the grid first reenergizing. Consumers must be closely involved in establishing the settings for the relay system. In particular, they will require under/over voliage and under/over frequency protection relaying. For undervoltage, the relay shall operate in less than 1 second if the voltage falls below $90 \%$ of line voltage. Comparably, the relay shall operate in less than 1 second for overvoltage events above $110 \%$. For overfrequency and underfrequency events falling outside the range of $58.5-60.5 \mathrm{Hertz}$ the relay shall operate within 0.2 seconds. Inverse time characteristics are not acceptable by Consumers. The project will also be required to manage its own internal faults. Direct Transfer Tripping will not be required as it is unlikely that the wind projects in question would use synchronous generators. This is good since DTT is typically an expensive configuration. 


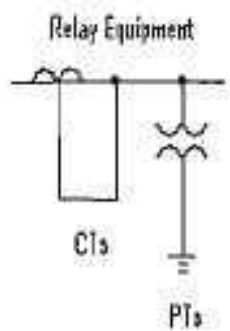

Figure 6, "Relay Equipment"

The wind project will be required to supply its own reactive power needs sufficient to maintain a steady state power factor of unity. This can be accomplished through switched capacitors. Consumers Energy does not currently require induction and inverter-type generators to supply reactive power into the grid beyond their own needs.

To study the stability of the local grid system after the introduction of the wind project, a fault current contribution of five times rated kVA of the wind project shall be used as a standard limit for design purposes. The interconnection study will determine whether or not the wind project meets this guideline.

The basic scope of the interconnection study will include all steady-state power flow, short circuit, and system stability analysis. The power flow analysis covers the basic thermal results of interconnecting the new generator. An analogy might be to think of the transmission system as a network of pipes, valves, pumps, etc. As additional water is inserted into the pipe network, an examination must be conducted to ensure that the existing elements are not overstressed. A typical analysis would include "system intact" as well as " $\mathrm{N}-1$ " conditions. System intact means that all elements are in service and functioning properly. $\mathrm{N}-1$ means that one element is not working, so the other parts must work harder to maintain the same system performance. This one element could be any element: transmission line, transformer, circuit breaker, etc.

The purpose of the short circuit analysis is to see what happens when a fault occurs. This is different than $\mathrm{N}-1$. Whereas in $\mathrm{N}-1$ something is simply taken out of service, in a short circuit situation current flows where it is not supposed to go. This generally means it will either flow from one phase to another (from one wire to another) or from one or more phases to ground. This can result in severe interruption of proper flows, so care must be taken to address these potential faults. Since local faults are typically the focus of this portion of the study, and since fault current contribution varies from one generator to the next, it is important that the specific wind turbine model be chosen no later than at this stage. It is also important to specify the intended power purchaser or "sink" for the study. That way system analyzers can schedule the output of the project to a specific utility or load in the models. 
The system stability analysis addresses the behavior of the wind project when there are faults on other parts of the transmission system. For example, if there is a fault on a line 200 miles away, will the wind project stay on-line or trip off-line? It will be at the discretion of the transmission service provider to decide whether it is desirable for the project to ride through a fault or briefly go off-line.

The final portion of the study is where Consumers makes estimates for the project as to the new or upgraded facilities required to interconnect the generator. The estimates will include both those facilities to be provided and owned by the project and those to be provided and owned by Consumers and/or other affected utilities.

Following the completion of the interconnection study, negotiations will begin on the Interconnection and Operating Agreement with Consumers. A copy of this agreement is available on the Consumers Energy website at www consumersencrgv.com.

\section{Markets}

As noted above, it is commonly beneficial to connect to the same utility that will buy the output of the project. Therefore, since the owner of the facilities at the two interconnection locations is Consumers Energy, it is the natural first choice for a power purchaser. Close second choices would be any of their others customers who could accept the power from the wind project into their remote systems even thought they would have to transmit the power over facilities owned by Consumers without paying transmission charges to Consumers. Third choices would be any utilities that could be reached by way of the Consumers Energy transmission system even transmission charges would be applied.

The rules for selling wind energy in Michigan are already fairly well established. If connecting to the distribution system, the sale would be to Consumers Energy at its thencurrent renewable energy rate. If connecting to the $46 \mathrm{kV}$ system or up, the power would be sold into the MISO market at a wholesale rate. This would increase the number of options for power purchasers for the Tribe's project(s), but it would not necessarily increase the price. In the case of distribution transformers where the high side is at least $46 \mathrm{kV}$, a project can connect to the low side of the transformer and still qualify for the wholesale market. This is the case with the Standish project. 


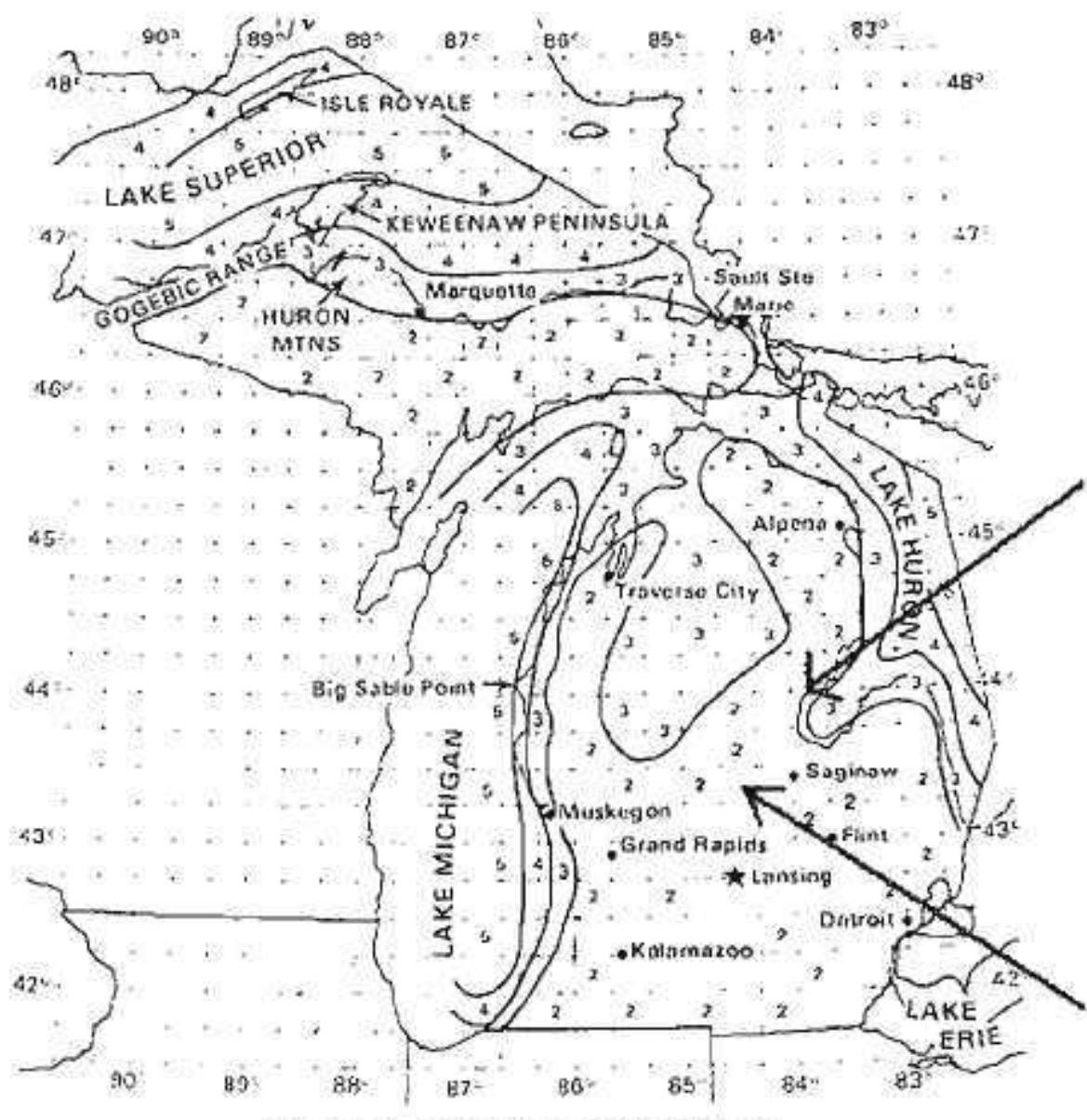

Figure 7, "Michigan Wind Map"

As shown in Figure 8 below, the State of Michigan does not currently have any large wind projects installed within its borders. However, there are three small projects installed that total $2.6 \mathrm{MW}$ of generation. $600 \mathrm{~kW}$ is installed near Traverse City, $1800 \mathrm{~kW}$ is installed near Mackinaw City, and $195 \mathrm{~kW}$ is installed in Huron County. At least two additional larger projects are proposed in Bingham and Oceana County for a total of $78 \mathrm{MW}$. However, even if these two projects are built the total installed wind capacity in Michigan will be less than $100 \mathrm{MW}$. Considering the fact that Michigan is ranked $14^{\text {th }}$ in the nation in terms of wind development potential and as evidenced by the Michigan state wind map in Figure 7, its present ranking in the lower half of the states in terms of wind projects installed suggests that there is a reasonable market for the purchase of wind energy. 


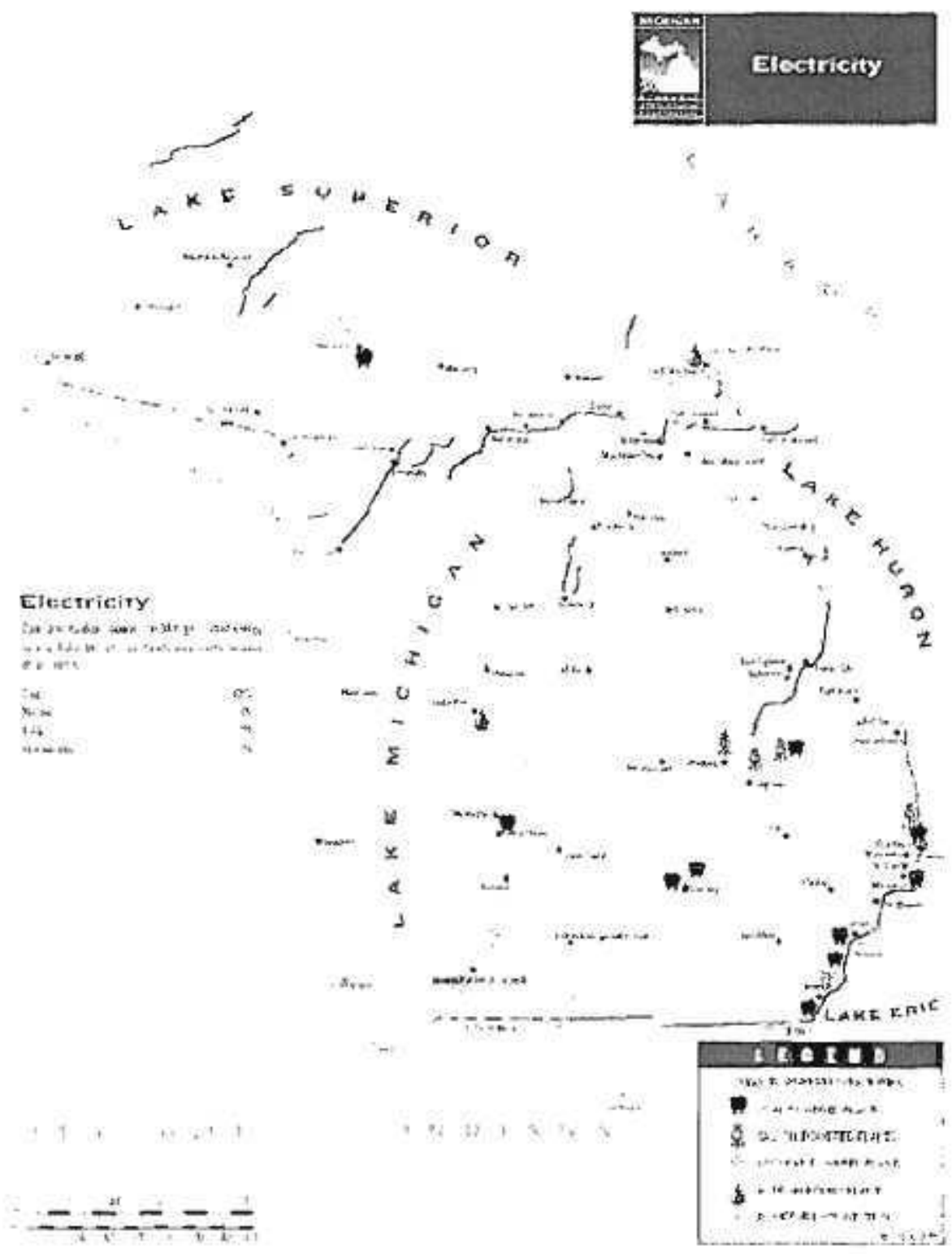

Figure 8, "Major Michigan Power Plants"

\section{Conclusion}

It is possible to avoid wasting months of time and thousands of dollars in the late development stages by making a few prudent decisions in the early stages. These include the selection of the interconnection point, target power purchaser, and basic project size. For the Standish site, a connection to one of the two distribution voltages $(24.9 \mathrm{kV}$ or $46 \mathrm{kV}$ ) is recommended at the Duquite Substation. The target project size should be in the neighborhood of $10 \mathrm{MW}$. For the Mount Pleasant site, a connection to the low side of the Casino's meter is recommended. The optimum project size has not been determined at this stage, but it will likely not exceed 5MW. For both projects Consumers Energy will be the target power purchaser. 
Disgen is confident of its abilities to guide the Tribe deftly and efficiently through the complicated interconnection process and thus help make a wind project on the Saginaw Chippewa Reservation a reality. 


\section{APPENDIX 1}

\section{INTERCONNECTION APPLICATION FORM}




\title{
GENERATOR INTERCONNECTION APPLICATION AGGREGATE GENERATOR OUTPUT OF 2 MW OR MORE
}

1. The undersigned Project Developer submits this Generator Interconnection Application and appropriate filing fee to interconnect a new Project to the Utility Electric System or to increase the capacity of an existing Project interconnected to the Utility Electric System.

2. A Project Developer requesting interconnection or an increase in the capacity of an existing Project to the Utility Electric System must provide the following information:

a. Completed Interconnection Application Data sheet appropriate for the capacity rating and type of generating unit(s), as found in the Utility's Generator Interconnection Requirements (Interconnection Application Data sheet, found in Appendix B or C, must be attached to this Interconnection Application).

b. Description of the equipinent configuration and proposed interconnection one-line diagram (one-line diagram must be attached to this Interconnection Application).

c. Project Developer (Single Point of Contact):

Name:

Address:

Phone Number.

Fax Number:

e-mail Address:

Project Site Address:

3. This Generator Interconnection Application shall be directed to the Utility representative as indicated below:

\author{
Director - Electric System Planning and Protection \\ Consumers Energy Company \\ 1945 West Parnall Road \\ Jackson, Mil 49201
}

4. 1. the undersigned and authorized representative of the Project, submit this Generator Interconnection Application and required technical data for the Utility's review. I understand that upon acceptance, the Utility shall subsequently provide an Interconnection Study Agreement. The Interconnection Study Agreement will include the Scope of the Interconnection Study. I also understand that I shail be required to furnish certain required technical data as requested by the Utility in support of this study and reimburse the Utility for its study expenses.

Authorized Signature:

Printed Name:

Title:

Company Name:

Date: 


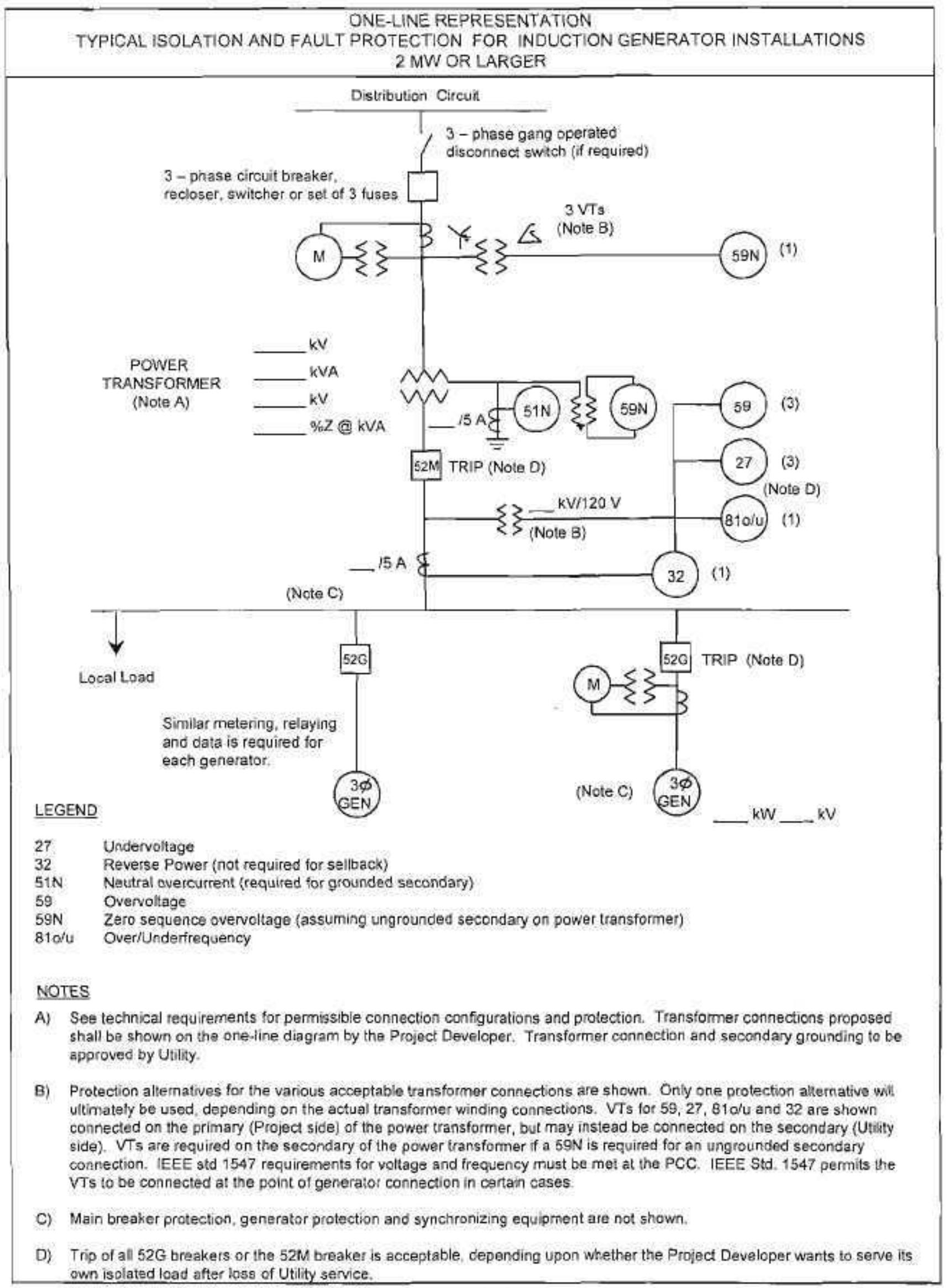




\section{SYNCHRONOUS OR INDUCTION GENERATORS - AGGREGATE $\geq 2 \mathrm{MW}$ INTERCONNECTION APPLICATION DATA FOR: \\ PROVIDED BY: \\ DATE:}

Instructions: Attach data sheets as required. Indicate in the tables below the page number of the attached data (manufacturer's data where appropriate) on which the requested information is provided. Provide one table for each unique transformer.

General Information

\begin{tabular}{|c|l|c|}
\hline $\begin{array}{c}\text { Item } \\
\text { No }\end{array}$ & \multicolumn{1}{|c|}{$\begin{array}{c}\text { Data } \\
\text { Description }\end{array}$} & $\begin{array}{c}\text { Attached } \\
\text { Page No }\end{array}$ \\
\hline 1 & Flow-back or Non-Flow-back & \\
\hline 2 & Project Type (Base load, peaking, intermediate) & \\
\hline 3 & Site Plan & \\
\hline 4 & Simple One-Line Diagram(s) for Project and Project Load & \\
\hline 5 & Detailed One-Line Diagram(s) for Project & \\
\hline 6 & Energization Date for Project Interconnection Facilities & \\
\hline 7 & First Parallel Operation Date for Testing & \\
\hline 8 & Project Commercial Operation Date & \\
\hline 9 & Estimated Project Cost & \\
\hline
\end{tabular}

\section{Isolating Transformer(s) between Project Developer(s) and Utility: Transformer} No

\begin{tabular}{|c|l|c|}
\hline $\begin{array}{c}\text { Item } \\
\text { No }\end{array}$ & \multicolumn{1}{c|}{\begin{tabular}{c}
\multicolumn{1}{c|}{$\begin{array}{c}\text { Data } \\
\text { Description }\end{array}$} \\
\hline 1
\end{tabular}} & $\begin{array}{c}\text { Attached } \\
\text { Page No }\end{array}$ \\
\hline 2 & kVA of each winding & \\
\hline 3 & BlL of each winding & \\
\hline 4 & Fixed taps available for each winding & \\
\hline 5 & Positiveinegative range for any LTC windings & \\
\hline 6 & $\%$ Impedance on transformer self cooled rating & \\
\hline 7 & Percent excitation current at rated kV & \\
\hline 8 & Load Loss Watts at full load or XR ratio & \\
\hline
\end{tabular}

The following information on these system components shall appear on the preliminary One-Line Diagram, including manufacturer make and model for the items listed below:

- Breakers - Rating, location and normal operating status (open or closed)

- Buses - Operating voltage

- Capacitors - Size of bank in KVAR

- Circuit Switchers - Rating, location and normal operating status (open or closed)

- Curtent Transformers - Overall ratio, connected ratio

- Fuses - normal operating status, rating (Amps), type

- Generators - Capacity rating (KVA), location, type, method of grounding

- Grounding Resistors - Size (ohms), current (Amps)

- Isolating transformers - Capacity rating (kVA), location, impedance, voltage ratings, primary and secondary connections and method of grounding

- Potential Transformers - Ratio, connection

- Reactors - Ohms/phase

- Relays - Types, quantity, IEEE device number, operator lines indicating the device initiated by the relays.

- Switches - Location and normal operating status (open or closed), type, rating

- Tagging Point - Location, identification 

SYNCHRONOUS GENERATORS - AGGREGATE $\geq 2$ MW
INTERCONNECTION APPLICATION DATA FOR:
PROVIDED BY:
DATE:

Instructions: Attach data sheets as required. Indicate in the table below the page number of the attached data (manufacturer's data where appropriate) on which the requested information is provided. Provide. one table for each unique generator.

Electric Generator(s) at the Project: Generator No

\begin{tabular}{|c|c|c|c|}
\hline $\begin{array}{l}\text { Item } \\
\text { No }\end{array}$ & $\begin{array}{l}\text { Data } \\
\text { Value }\end{array}$ & $\begin{array}{c}\text { Data } \\
\text { Description }\end{array}$ & $\begin{array}{l}\text { Attached } \\
\text { Page No }\end{array}$ \\
\hline 1 & & Generator Type (synchronous or induction) & \\
\hline 2 & & Generator Nameplate Voltage & \\
\hline 3 & & Generator Nameplate Watts or Volt-Amperes & \\
\hline 4 & & Generator Nameplate Power Factor (pf) & \\
\hline 5 & & RPM & \\
\hline 6 & & Minimum and Maximum Acceptable Terminal Voltage & \\
\hline 7 & & Direct axis reactance (saturated) & \\
\hline 8 & & Direct axis reactance (unsaturated) & \\
\hline 9 & & Quadrature axis reactance (unsaturated) & \\
\hline 10 & & Direct axis transient reactance (saturated) & \\
\hline 11 & & Direct axis transient reactance (unsaturated) & \\
\hline 12 & & Quadrature axis transient reactance (unsaturated) & \\
\hline 13 & & Direct axis sub-transient reactance (saturated) & \\
\hline 14 & & Direct axis sub-transient reactance (unsaturated) & \\
\hline 15 & & Leakage Reactance & \\
\hline 16 & & Direct axis transient open circuit time constant & \\
\hline 17 & & Quadrature axis transient open circuit time constant & \\
\hline 18 & & Direct axis subtransient open circuit time constant & \\
\hline 19 & & Quadrature axis subtransient open circuit time constant & \\
\hline 20 & & Open Circuit saturation curve & \\
\hline 21 & & $\begin{array}{l}\text { Reactive Capability Curve showing overexcited and } \\
\text { underexcited limits (Reactive Information if non-synchronous) }\end{array}$ & \\
\hline 22 & & $\begin{array}{l}\text { Excitation System Block Diagram with values for gains and time } \\
\text { constants (Laplace transtorms) }\end{array}$ & \\
\hline 23 & & $\begin{array}{l}\text { Short Circuit Current contribution from Generator at the Point of } \\
\text { Common Coupling }\end{array}$ & \\
\hline 24 & & $\begin{array}{l}\text { Rotating inertia of overall combination Generator, prime mover, } \\
\text { couplers and gear drives }\end{array}$ & \\
\hline 25 & & Station Power load when generator is off-line, Watts, pf & \\
\hline 26 & & Station Power load during start-up, Watts, pf & \\
\hline 27 & & Station Power load during operation, Watts, of & \\
\hline 28 & & $\begin{array}{l}\text { National Recognized Testing Laboratory Certification (if } \\
\text { applicable) }\end{array}$ & \\
\hline 29 & & Written Commissioning Test Procedure & \\
\hline
\end{tabular}




\section{INDUCTION GENERATORS - AGGREGATE $\geq 2$ MW \\ INTERCONNECTION APPLICATION DATA FOR: \\ PROVIDED BY:}

DATE:

Instructions: Attach data sheets as required. Indicate in the table below the page number of the attached data (manufacturer's data where appropriate) on which the requested information is provided. Provide one table for each unique generator.

Electric Generator(s) at the Project:

\begin{tabular}{|c|c|c|c|}
\hline $\begin{array}{l}\text { Item } \\
\text { No }\end{array}$ & $\begin{array}{l}\text { Data } \\
\text { Value }\end{array}$ & $\begin{array}{c}\text { Data } \\
\text { Description }\end{array}$ & $\begin{array}{l}\text { Attached } \\
\text { Page No }\end{array}$ \\
\hline 1 & & Generator Type (synchronous or induction) & \\
\hline 2 & & Generator Rated Voltage & \\
\hline 3 & & Generator Rated Volt-Amperes & \\
\hline 4 & & Generator Rated Power kW & \\
\hline 5 & & Number of Poles & \\
\hline 6 & & Synchronous Rotational Speed & \\
\hline 7 & & Rotation Speed at Rated Power & \\
\hline 8 & & Slip at Rated Power & \\
\hline 9 & & Minimum and Maximum Acceptable Terminal Voltage & \\
\hline 10 & & Motoring Power (kW) & \\
\hline 11 & & Neutral Grounding Resistor (If Applicable) & \\
\hline 12 & & $\mathrm{I}_{2}{ }^{2} \mathrm{t}$ or $\mathrm{K}$ (Heating Time Constant): & \\
\hline 13 & & Rotor Resistance & \\
\hline 14 & & Stator Resistance & \\
\hline 15 & & Stator Reactance & \\
\hline 16 & & Rotor Reactance & \\
\hline 17 & & Magnetizing Reactance & \\
\hline 18 & & Short Circuit Reactance & \\
\hline 19 & & Exciting Current & \\
\hline 20 & & Temperature Rise & \\
\hline 21 & & Frame Size & \\
\hline 22 & & Design Letter & \\
\hline 23 & & Reactive Power Required in Vars (No Load) & \\
\hline 24 & & Reactive Power Required in Vars (Full Load) & \\
\hline 25 & & Total Rotating Inertia, $\mathrm{H}$ : $\quad$ Per Unit on KVA Base- & \\
\hline 26 & & $\begin{array}{l}\text { Short Circuit Current contribution from generator at the Point of } \\
\text { Common Coupling }\end{array}$ & \\
\hline 27 & & $\begin{array}{l}\text { Rotating inertia of overall combination generator, prime mover, } \\
\text { couplers and gear drives }\end{array}$ & \\
\hline 28 & & Station Power load when generator is off-line, Watts, pf & \\
\hline 29 & & Station Power load during start-up, Watts, pf & \\
\hline 30 & & Station Power load during operation, Watts, pf & \\
\hline 31 & & $\begin{array}{l}\text { National Recognized Testing Laboratory Certification (if } \\
\text { applicable) }\end{array}$ & \\
\hline 32 & & Written Commissioning Test Procedure & \\
\hline
\end{tabular}


Appendix M. "Power Point" Presentation Slides 
Appendix M. "Power Point" Presentation Slides 


\section{Saginaw Chippewa Indian Tribe}

\section{July 28, 2008}

\section{Final Report}

\section{Renewable Energy Feasibility Study}

Wind - Solar - Biomass

US Department of Energy Grant

Presented by Don Seal, Tribal Planning Eng.

Sally Kniffen, Tribal Environmental Steward

Steven B. Smiley, Consultant- Renewable Energy Economist 


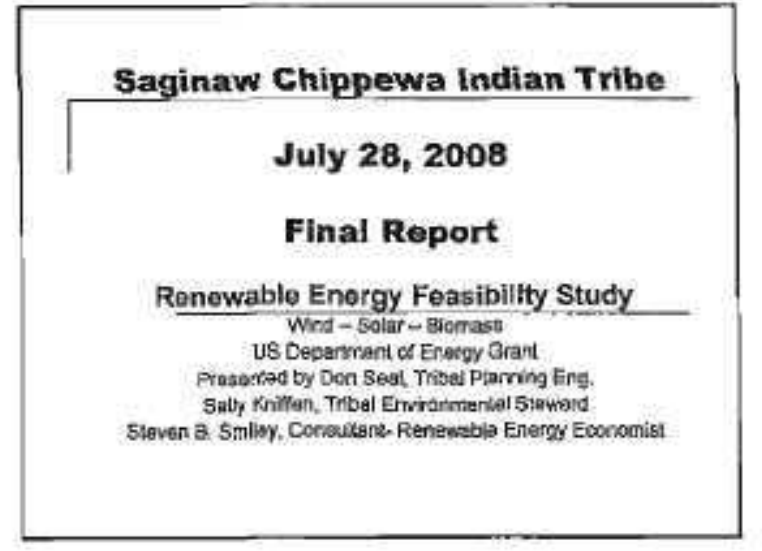

"An Inconvenient Truth" Al Gore Illuminates the PROBLEM

- The Greenhouse Effect: Greenhouse gases

c. Highest $\mathrm{CO} 2$ levels 850,000 years and past

- Highest temperatures in 1000 year ice core

a USA emissions up $20 \%$

No doubt, we're in deep, deep, do-do...

\section{The Vision*}

- The Tribe is secking to become selt-sufficient in their. energy needs,...

- The Tribe wishes to maintain its culture by protecting Mother Earth and look ahead seven generatons...

* Green energy sources are the best way...

- The Tribe's objectives are to provide power for its facilifies and potentially create economic development and empioyment opportunitios in its depressed arous ...

- 'These efforts are to halp the Trba beconse sufficient in providing their own energy for the various bulidings. cuitural canters, schools and residences on Tribe land,"

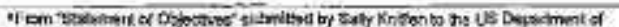
Enepl. vay 2004
Hermann Scheer tluminates the SOLUTION: "Our farmers will be the oll sheiks af tomorrow."

The samern wit become comdinoc tooc, enemyy and row mglerial producats, and they will bs acologically integreted. Ruralisation wil lad ic now forms of sextemans and revifulteotion of the preniay oconcriny with herasos lecal enployment end tass continuting

Puthcatons:

A Solar Manifosto

The Solter Economy, \& Eisargy Autonomy.

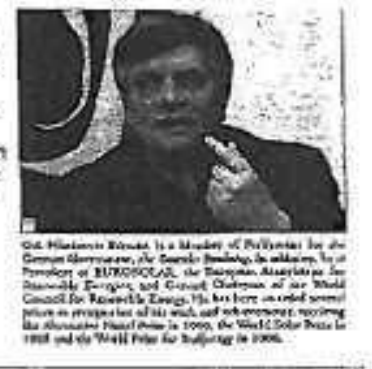

\section{The Problem}

The Seven Crises Related to Nuclear 8 Fossit Energy Use

- The global climate crisis

- The exhaustion \& dependence crisis

w The poverty crisis

- The nuclear crisis

- The water crisis

- The farming crisis

* The health crisis

\section{Global energy Resources}

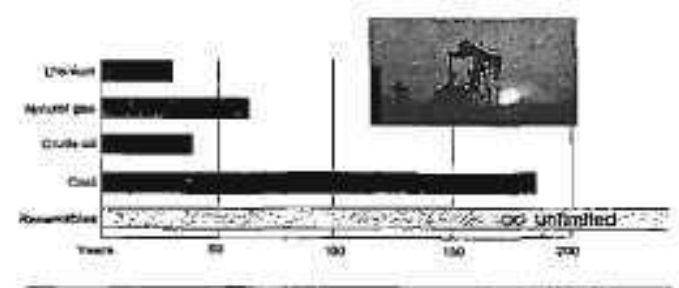



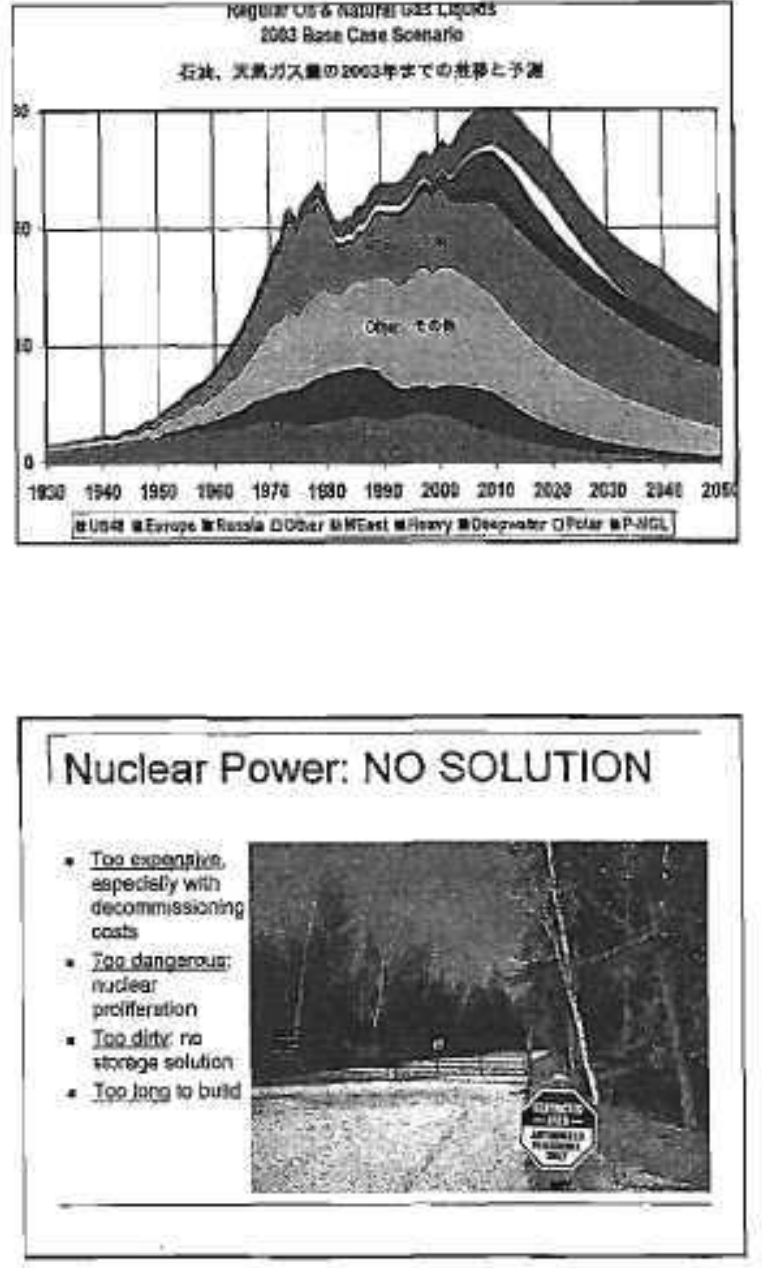

Towards A $100 \%$ Renewable Energy Economy

- Local Production of energy

- Creation of jobs in the local economy

- Improved Living Conditons

- Industrial devolopment, new Technologies

- Soil Conservation

* Saving Foreign Currencies

- Replacement of Fossil \& Nuciear Fuels

- Climatíc and Environmental Benefits

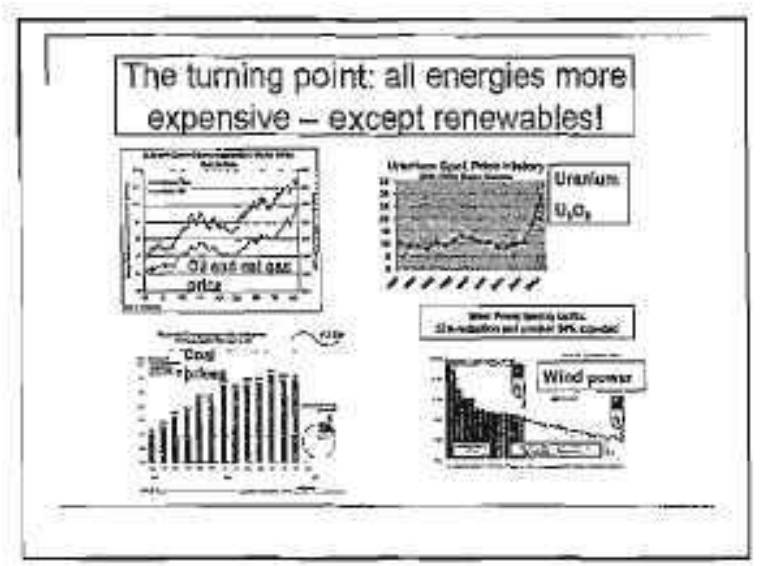

Nuclear Power and Coal in NW Lower Michigan: Going, going, GONE

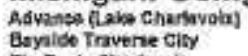
Big Rock, Charievoix.
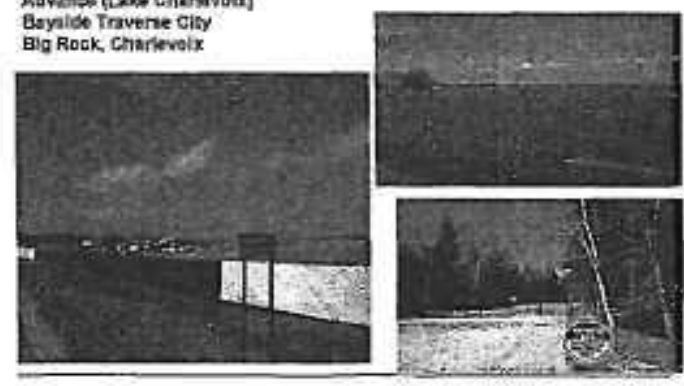

Energy Autonomy: The New Standards

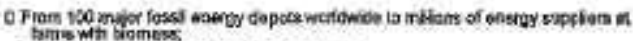

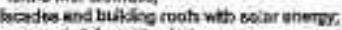

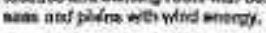

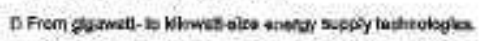

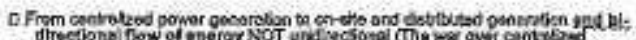

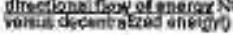

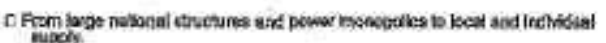
iupoty.

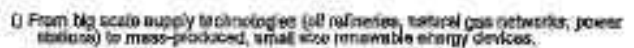


The Conceptuai New Energy Approach for the Tribe: Integrated Renewables

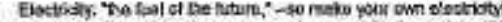

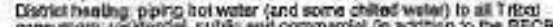

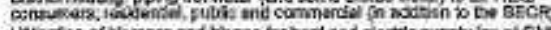

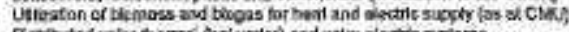

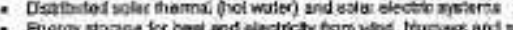

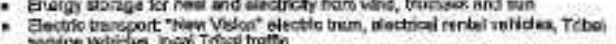

- Esurgy managamast ans controb for traes opatome

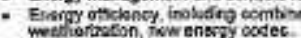

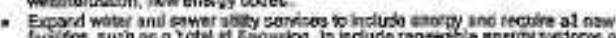

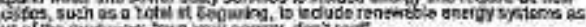

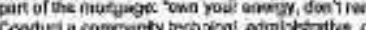

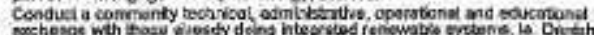
neighbors.
Electricity: "The fuel of the future"

* Electricity infrastructure is in place and can be upgraded easily

- Organized and efficient distribution

- Electricty provides flexibility with low-cost controls and storage, including options for elecaric storage using hot water from wind power and other batterles

- Electric vehicles will repiace gasoline and other fossid fue! powared engines, including the "New Vision" electric tram, electric service vehicles; tentals for visitors and other local transportation
District Heat: "The heat distribution system of the future"

- District Heat from Biomass (sustainable wood waste, wood chips, agricultural waste, etc. replaces natural gas)

- Distribution of hot water is metered just like olectricity and water

- Will replace $>1000$ fossil fuel chimneys with two chimneys-with much lower environmental impacts

- Significantly eliminate homeowner and Tribal furnace and boiler service costs

\section{Tribe Profile}

w Tribe member population $~ 3,300$

- Isabella 138,240 ac total area (1788 ac in trust)

- Saganing 500 acres (184 ac in trust)

- Residential: -500 homes and apartments (trust and non-trust lands) $\sim 600,000$ sq.ft.

- Public Facilties: $\sim 24 \sim 320,000$ sq. ft.

- Commercial; $5+\sim 550,000$ sq. $\mathrm{ft}$.

\section{Energy Profile}

\section{Summary:}

Total Residential, Public \& Commercial Cost: $\$ 5,412,646 / y r$

Electric: $\$ 3.6$ millionlyr - 51 million kilowatt- hrs Peak Electric Demand: $8,000 \mathrm{~kW}$

Natural Gas: $\$ 1.8$ million/yr -1.8 million ccPs per year

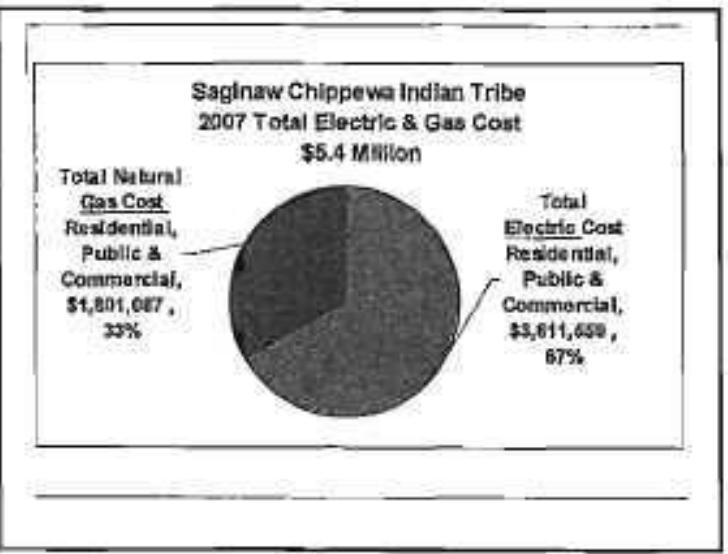



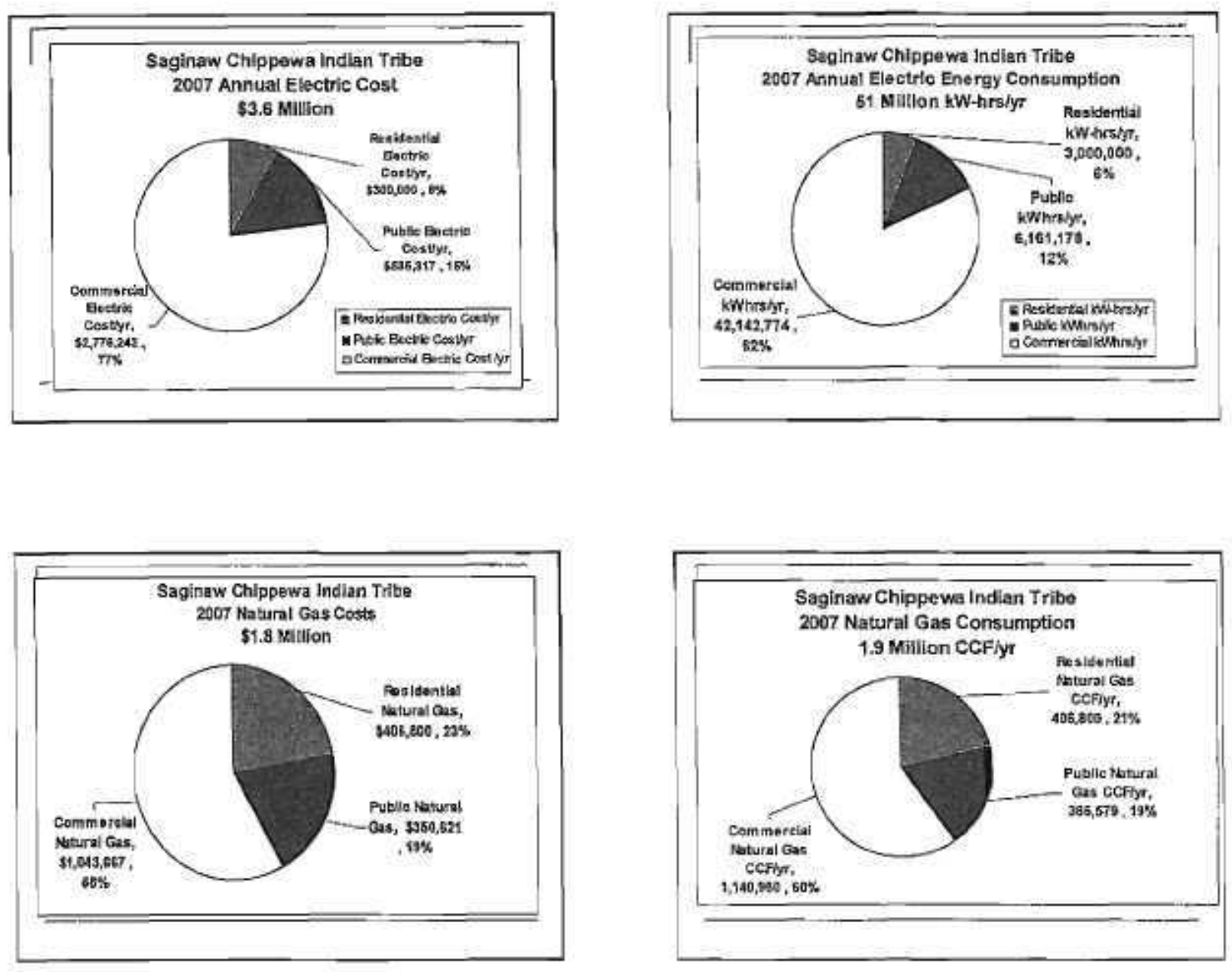

\section{Energy Profile 2007}

\section{Electric}

\begin{tabular}{|c|c|}
\hline Residertial Electric Use: & $2,000,000$ KWhnyit \\
\hline Public Electrie Use: & B, 161,978 W/hoirt \\
\hline Cemmertial Electric Use: & 42.143774 k7mentr \\
\hline Total $R, P$ \& Commerclal & B1,303,082 kWhityt \\
\hline $\begin{array}{l}\text { Resiflential Electric Cost: } \\
\text { Publie Electric Cost: } \\
\text { Coemmerclal Electrie Cost: }\end{array}$ & 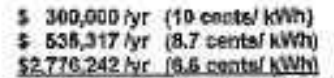 \\
\hline Total R, P \& C Electric Cost: & $53,611,589$ /yz (7 centes/hWh avp \\
\hline
\end{tabular}

\section{Energy Profile}

\section{Natural Gas}

Fosidentiel Naturat cas Poble Naterat Gae

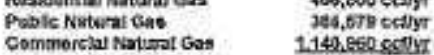
Total Noturel cas

Residential Natureil Oas Cocs Pable Naturat Gas Cest Commercial Nateral Oat Cost \$1.043.667 /ar is.91/ ce. Total Nutaral Ges Cest
250.621 fyr is.96 toc

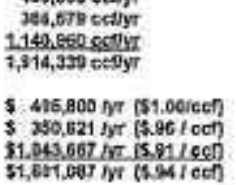




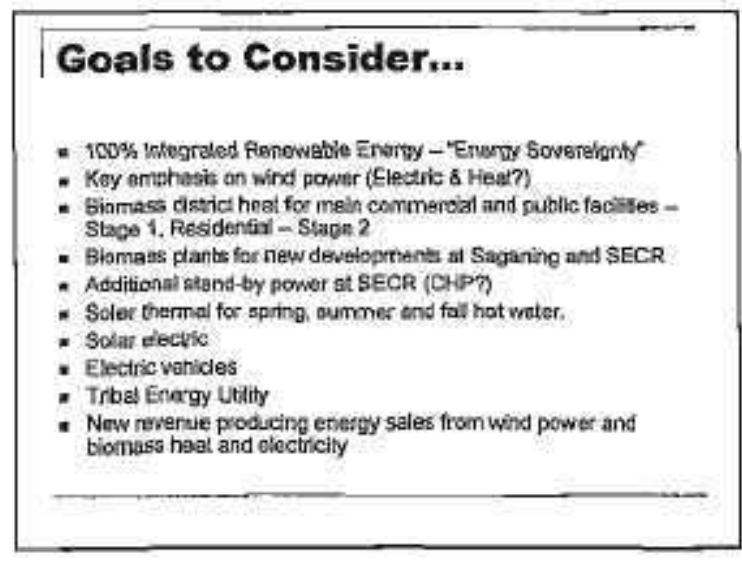

\section{The Renewable Resources}

- Wind resources at Isabella and Saganing sites

- Solar resources

*iomass and biogas resources

\section{Technologies Analyzed}

a Wind Power; small and large commercial (including wind farms)

- Biomass: small scale and large scale district heat

* Solar Thernal small and large scale

- Solar Electric (photovoltaic): small and large

- Natural gas combined heat \& power with SECR stand-by capacity

\section{Wind and Solar Comparison}

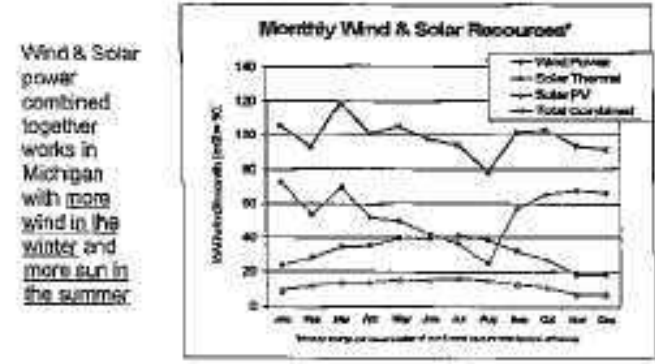

Isabella \& Saganing Wind Met Towers (30, 40 \& 50 meter measurements)
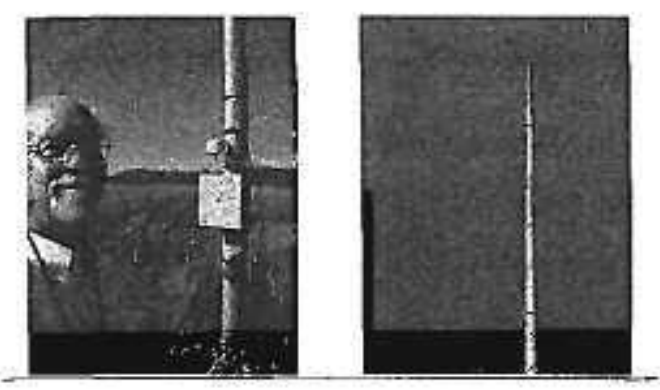

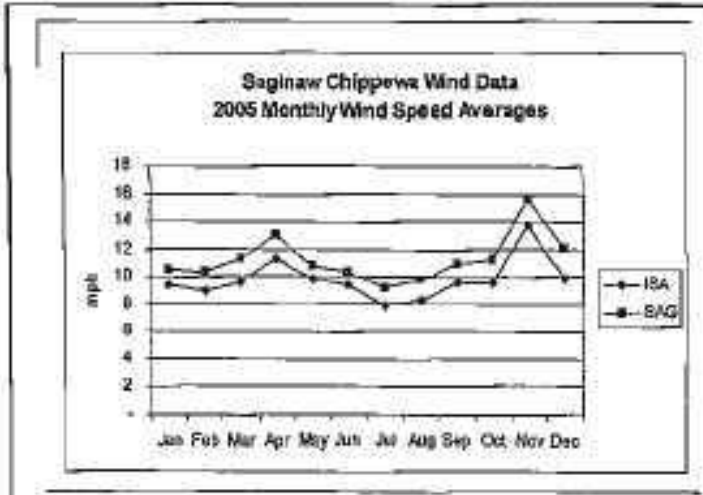



Detalled Wind Data Analysis: Saganing 2005 Summary results

- Mensured mean whd apeed \& 50 melors: $5.1 \mathrm{~m} / \mathrm{s}$ or $11,42 \mathrm{mph}$ (west sarsor boom)

- Estimeted conservitive wind shage axponent $=0.2$ (actual megsiraments from $B$ sensor comparisons ranged fom 0.17 to 35 with avarage of 26 )

- Annual mean wind spreed as 80 meters (262 $R$ ) (Abovs Ground leve') AGL: $6.2 \mathrm{mb}$ or $139 \mathrm{mph}$

- Annual meen wind speed es 100 metars (320 fit) AGL: $14.6 \mathrm{mph}$

- Wind power density lor standard Cless ili wind turbine:

a 80 m AGL $-613 \mathrm{kWh} / \mathrm{m} 2 \mathrm{hy}$

100 in AGL - 8G2 $\times$ Whutrongy

- NCAR (Nailonal Centor..) Data inditates 2005 wos ons of lowes:

wind yenrs in repion since 1998

- Therefore: Wend energy output projections, and resulting

econotrics from this msarured data stiauld be conaervolive

-

Detailed Wind Data Analysis: Isabella 2004-08

Summary results

- Messirred mesn wind spaed at 50 melers: $4.5 \mathrm{~m} / \mathrm{s}$ or $10.03 \mathrm{mah}$ (viest sensor boom ₹1)

- Estimaied conservative wind shaer axponent $=0$.

- Annual mean wind apeed et ed meters (262 fi.) AGL: $5.4 \mathrm{mva}$ or $12.0 \mathrm{mph}$

- Annual mean wird specd al 100 meters (328 n) ACL: $5.8 \mathrm{mts}$ or 13 mph

- Wrif powes densily for standerd Cases HI wind turbine. - $60 \mathrm{~m}$ AGL - $480 \mathrm{kWhrin} 2 / \mathrm{yr}$

- $100 \mathrm{~m} \mathrm{AGL}-560$ est. KWhilme2/yt

- NCAR (Nafiond Center.j Detrindicales 2005 was one of lewest wind yobs in regian shos 19ga

- Therefuce: Wind enerry output projectoris, and retrulting. econcontrs fram this incesuned da a sheulid be ccriservativo
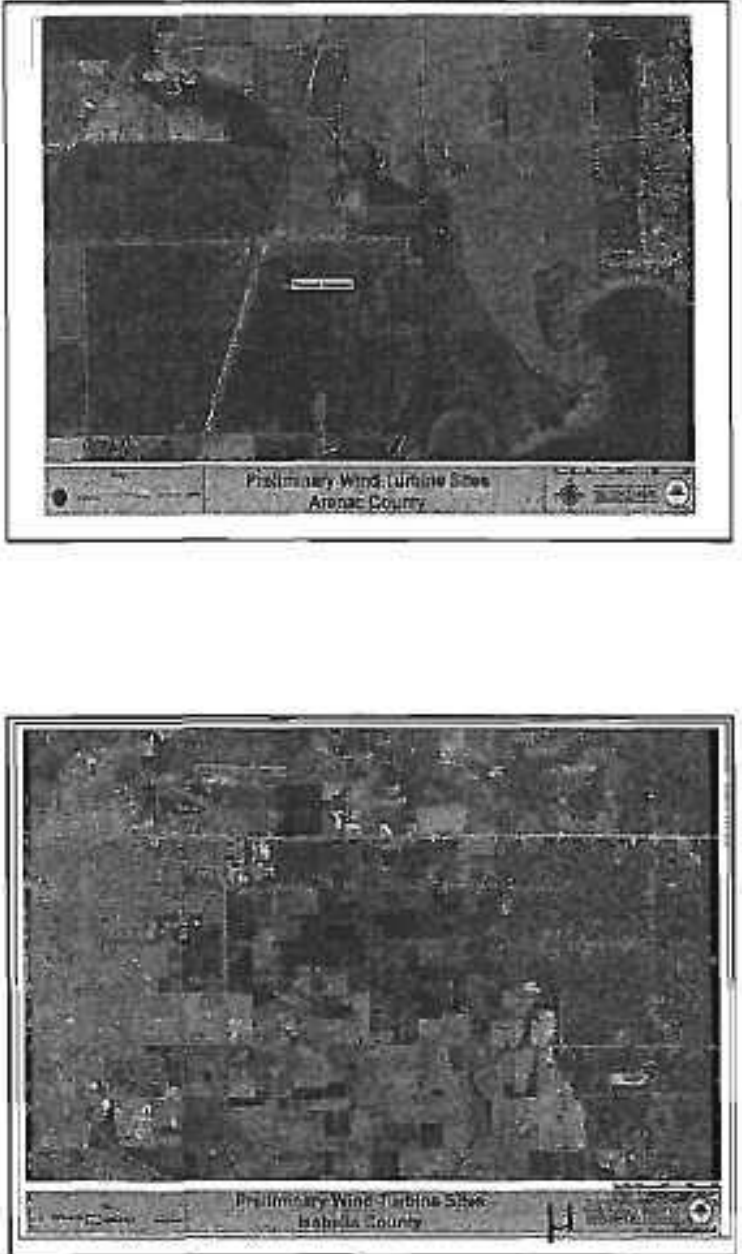

Saganing Wind Project Economics:

13 Wind Tutines

Tolal Cost 535

Milion

- Gross income per year \$4 million

- Cost of Eloctricity 4.5 cents per kWh

- Net Prosent Value: $\$ 41$ milion
Isabella Wind Project:

19 Wind Turbines

- Total Project Cost: $\$ 48$ Malion

- Gross income per year: $\$ 5.1$ milion

- Cost per KWh: 5.7 cents $/ \mathrm{kWh}$

- Net Present

Value: $\$ 39.3$ millian

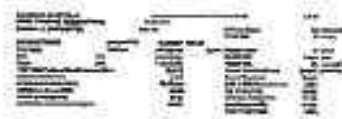

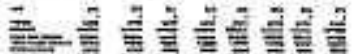

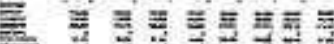

政

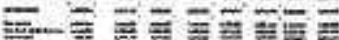

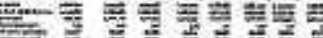

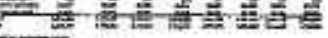

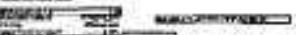




\begin{tabular}{|c|c|}
\hline \multicolumn{2}{|c|}{ 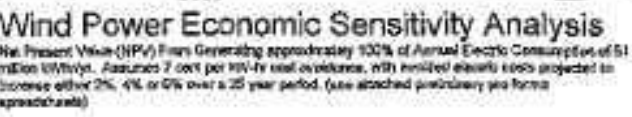 } \\
\hline \multicolumn{2}{|c|}{ Seganing: 13 Wind Tuthes - Project Cost - $\$ 34,998,000$} \\
\hline Anoidad & \\
\hline Cost infation & NPV $(25$ yn \\
\hline $2 \%$ & $\$ 20,047,200$ \\
\hline $4 \%$ & $541,008,046$ \\
\hline $6 \%$ & $\$ 64,100,500$ \\
\hline \multicolumn{2}{|c|}{$\begin{array}{c}\text { Isabeles: } 18 \text { Wind Turbinas - Prolec Cost - } \$ 49,780,000 \\
\text { NPV (25 yr) }\end{array}$} \\
\hline $2 \%$ & $517,975,002$ \\
\hline $1 \%$ & $\$ 39,323,111$ \\
\hline $6 \%$ & $\$ 68,302,851$ \\
\hline
\end{tabular}

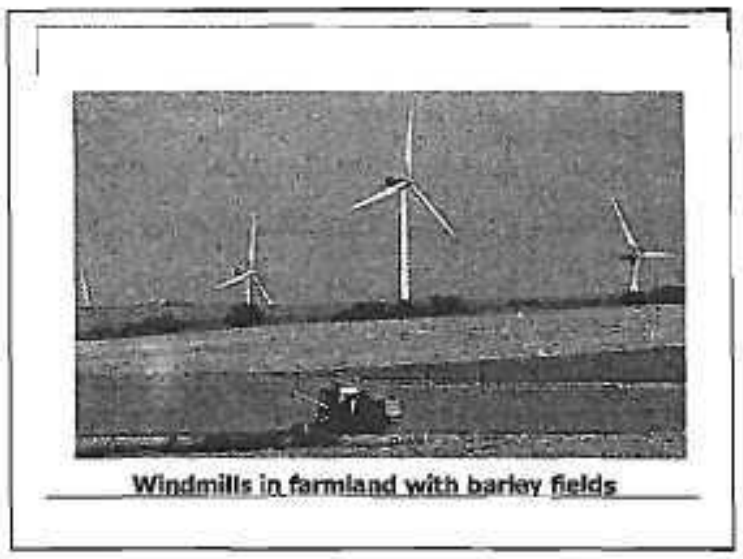

\section{Biomass District Heat}

- Main fuel source: wood chips, delivered in semi-tractor trailers ( 30 to 40 tons per load)

- Isabella County has 35,000 cubic yards per year

* Established market for delivery @\$20 - \$26 perton

- CMU has operation of district heat and electric steam turbine plant since mid 1980's. is There Enough Wind Energy? S. Thy, Denmark: $130 \%$ Wind Power, $88 \%$ Blornass Population 10,000 +
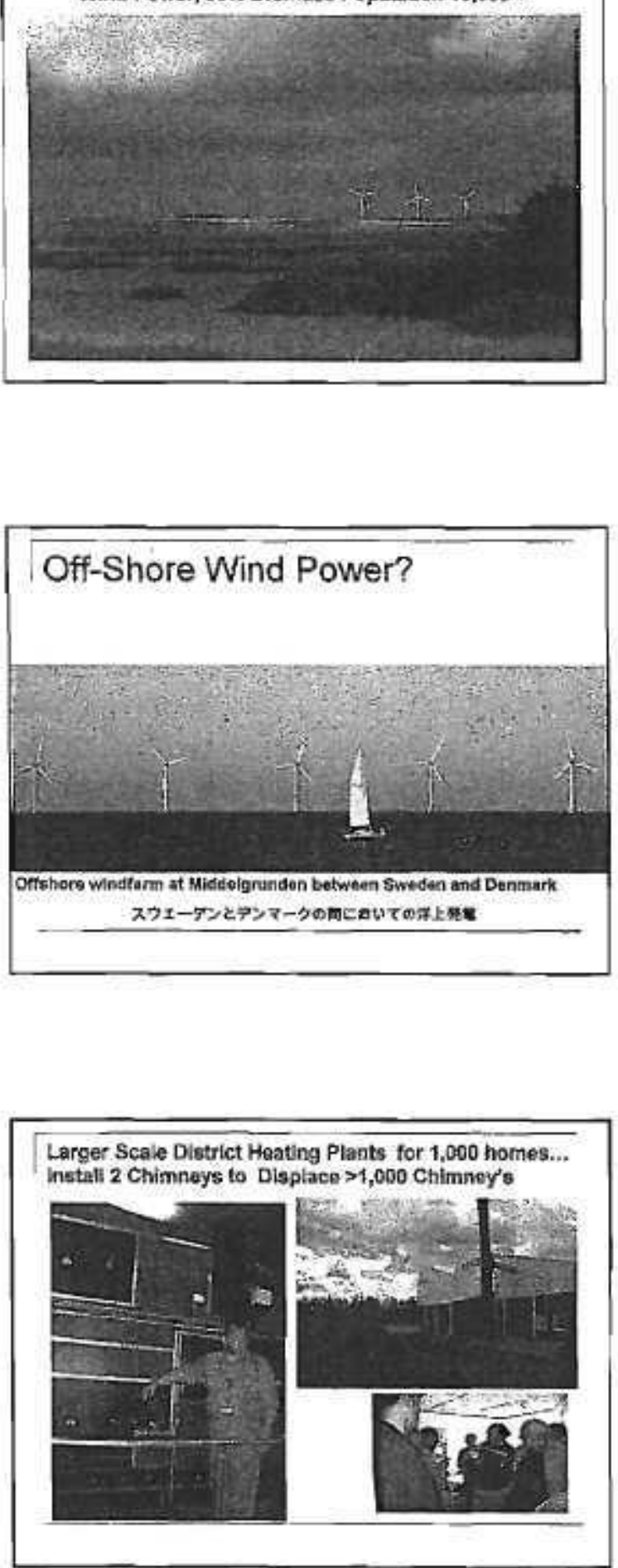

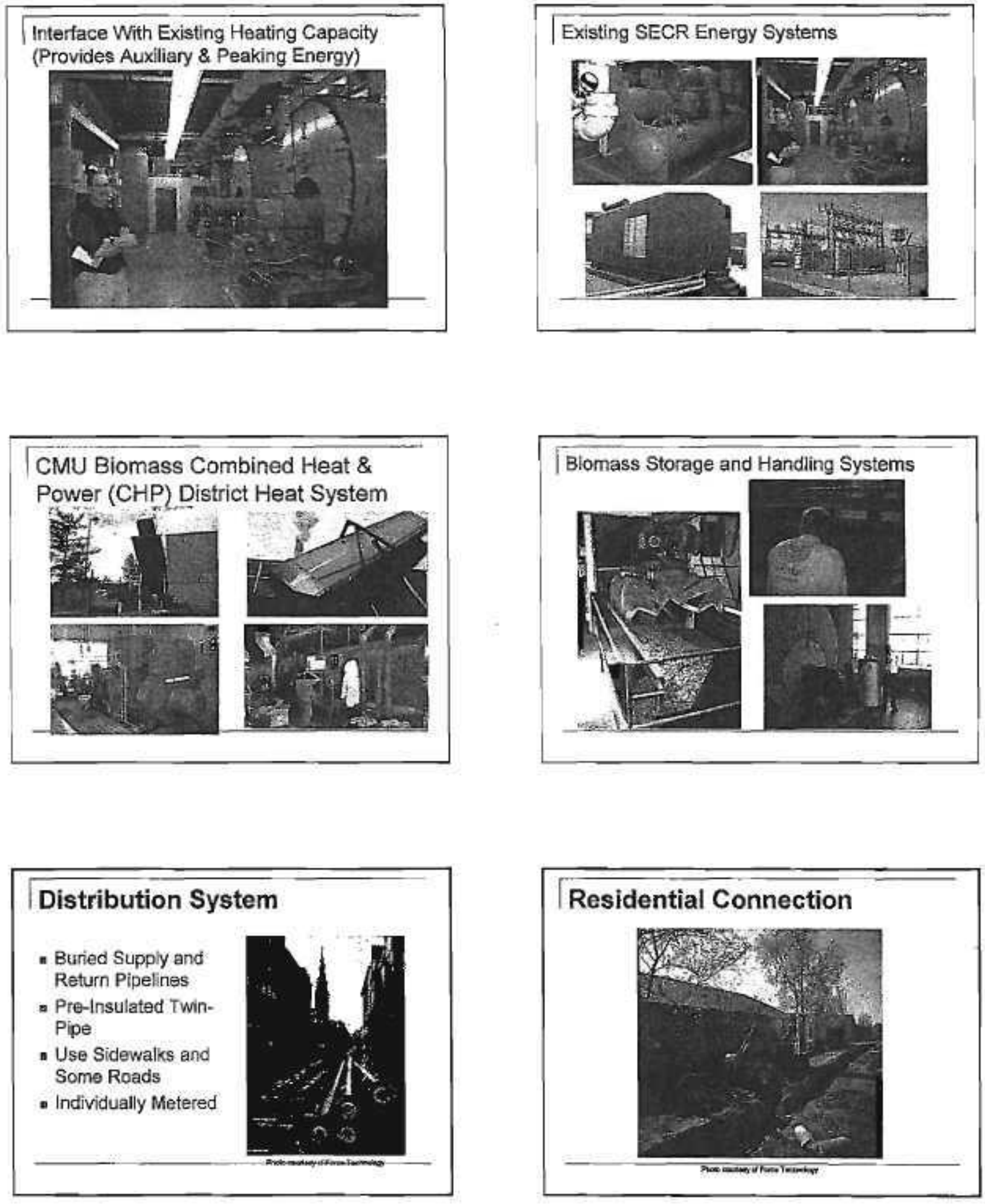

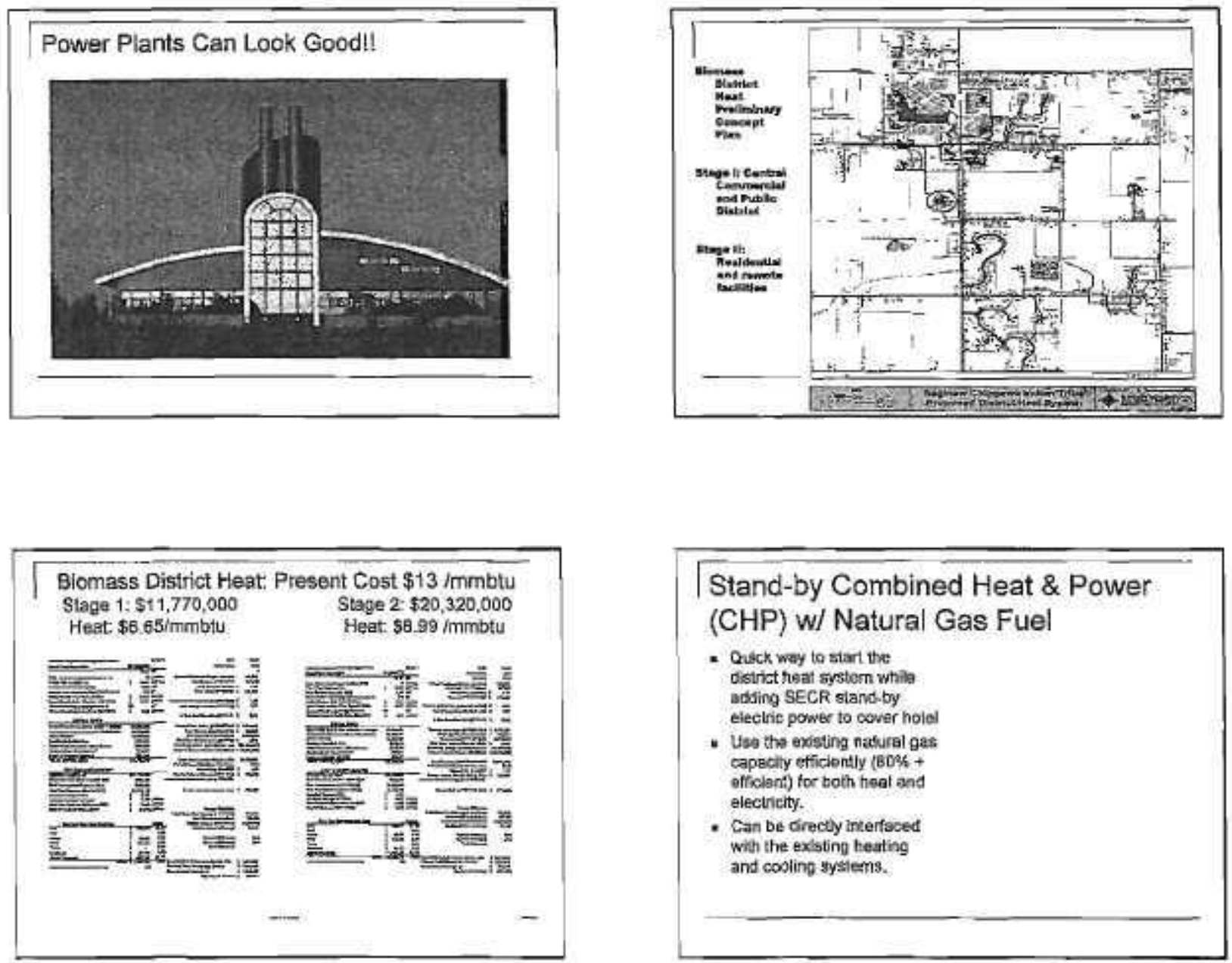

\section{Stand-by Combined Heat \& Power} (CHP) w/ Natural Gas Fuel

- Qusck way to siart the district reat systom while adding SECR sland.by electric power to cover hoiel

- Usa the exosting natural gas capecily efficiontly (BOSS + officlant) for both heat and electricity.

- Can be drectly interfeced with the extsting heating and cooling systems.

\section{CHP Natural Gas Stand-by Con't}

- Installed cost \$2.4 million (wib heat or chilled water storage)

- Peak electsic capacity 3,500 to $4,000 \mathrm{~kW}$ (depending on sysiem choice) from two or three gas reciprocating engines)

- May recuire additional heat and possible chilled water storage (approx: $\$ 600,000$ ) with total of $\$ 2.8$ milion

* Using same amount of present natural gas consumption. generate $25 \%$ of SECR electricity, $2 / 3$ rds of heat and save $\$ 500,000$ per year while having $100 \%$ SECR standby electric capacity

- Provide infrastructure basis for biomess CHP installation and diafrict heat system.

\section{Solar Thermal}

a Smali systems for each home and building - Large commercial systerns for casino resort

- A large solar thermal system can provide most of the "domestic hot water and process water (cleaning, etc.) for the casino and hotel to displace natural gas) 


\section{Solar Thermal: Commercial SECR}

- Estimated Cost: $\$ 4.8$ million

- Annual Natural Gas Savings: $\$ 400,000$

- Large array on parking deck or adjacent field of up to $200,000 \mathrm{sq}$. $\mathrm{ft}$.

- Provide nearly $100 \%$ of domestic hot water for SECR during spring, summer and fall.

\section{Solar Electric}

a Small and large systems

a Home, commercial and public applications based on the solar resource

- Most expensive, but most reliable eiectric power source. More analysis to follow.

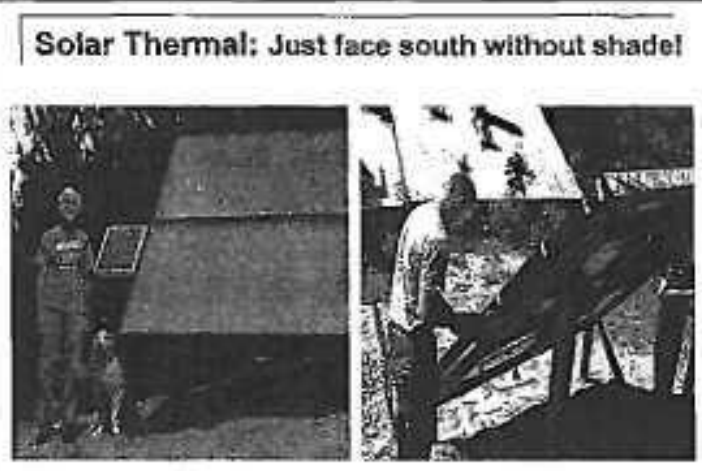

Solar electric (photovoltaics): Peak power when we need it.

- Off Grid

* Hybrid Grid

< Direct interconnect

- Net metering

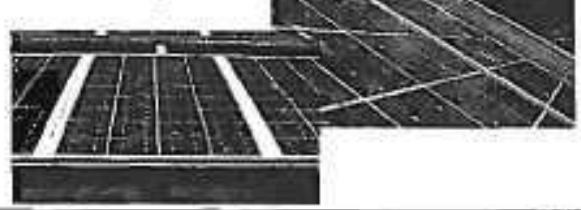

\section{Next Steps}

w Continuing tasks under the grant

- Guidance from the Counci 


\begin{tabular}{|c|c|c|c|c|c|c|c|c|c|}
\hline \multirow{2}{*}{$\begin{array}{l}\text { Ranked By Lowest to Highest } \\
\text { Efficiency /Passive Solar }\end{array}$} & \multirow{2}{*}{$\begin{array}{l}\text { Unit } \\
\mathrm{kW} \text {-hr }\end{array}$} & \multirow{2}{*}{\multicolumn{2}{|c|}{$\$ \begin{array}{l}\text { Unit } \\
\text { Cost }\end{array}$}} & \multicolumn{2}{|c|}{$\begin{array}{l}\text { Energy Only } \\
\text { Cost } / \mathrm{kWh}\end{array}$} & \multicolumn{2}{|c|}{$\begin{array}{l}\text { All Costs } \\
/ \mathrm{kW} \text {-hr }\end{array}$} & \multicolumn{2}{|c|}{$\begin{array}{l}\text { W/Enviro } \\
\text { Costs/kWh }\end{array}$} \\
\hline & & & & $\$$ & - & $\$$ & 0.03 & $\$$ & 0.03 \\
\hline Wood Chips & US Ton & $\$$ & 26.00 & $\$$ & 0.007 & $\$$ & 0.03 & $\$$ & 0.04 \\
\hline Large Wind & $k W-h r$ & $\$$ & - & $\$$ & - & $\$$ & 0.06 & $\$$ & 0.06 \\
\hline Cord Wood & Face Cord & $\$$ & 70.00 & $\$$ & 0.065 & $\$$ & 0.06 & $\$$ & 0.07 \\
\hline Dryed Cherry Pits or Pellets & Ton & $\$$ & 200.00 & $\$$ & 0.067 & $\$$ & 0.08 & $\$$ & 0.08 \\
\hline Natural Gas CHP (electric) & $\mathrm{CCF}$ & $\$$ & 1.20 & $\$$ & 0.055 & $\$$ & 0.07 & $\$$ & 0.09 \\
\hline Natural Gas CHP (heat) & $\mathrm{CCF}$ & $\$$ & 1.20 & $\$$ & 0.055 & $\$$ & 0.07 & $\$$ & 0.09 \\
\hline Natural Gas Large & $\mathrm{CCF}$ & $\$$ & 1.20 & $\$$ & 0.055 & $\$$ & 0.08 & $\$$ & 0.10 \\
\hline Naturai Gas Res /Comm & $\mathrm{CCF}$ & $\$$ & 1.25 & $\$$ & 0.057 & $\$$ & 0.09 & $\$$ & 0.11 \\
\hline Lg Commercial Gri & kW-hr & $\$$ & 0.070 & $\$$ & 0.070 & $\$$ & 0.09 & $\$$ & 0.13 \\
\hline Solar Hot Water & $\mathrm{kW}-\mathrm{hr}$ & $\$$ & $=$ & $\$$ & - & $\$$ & 0.15 & $\$$ & 0.15 \\
\hline Sm Commercial EI & kW-hr & $\$$ & 0.100 & $\$$ & 0.100 & $\$$ & 0.11 & $\$$ & 0.15 \\
\hline Residential Electricity & kW-hr & $\$$ & 0.100 & $\$$ & 0.100 & $\$$ & 0.11 & $\$$ & 0.15 \\
\hline LP Gas & Gallons & $\$$ & 2.50 & $\$$ & 0.121 & $\$$ & 0.14 & $\$$ & 0.18 \\
\hline New Coal Fired Electricity & $\mathrm{kW}$-hr & $\$$ & 0.170 & $\$$ & 0.170 & $\$$ & 0.17 & $\$$ & 0.19 \\
\hline Gasoline & Gallons & $\$$ & 3.90 & $\$$ & 0.160 & $\$$ & 0.18 & $\$$ & 0.22 \\
\hline Heat & Gallons & $\$$ & 4.85 & $\$$ & 0.162 & $\$$ & 0.18 & $\$$ & 0.23 \\
\hline New Atomic Electricity & $\mathrm{kW}-\mathrm{hr}$ & $\$$ & 0.23 & $\$$ & 0.230 & $\$$ & 0.23 & $\$$ & 0.25 \\
\hline Small Wind & kW-hr & $\$$ & - & $\$$ & - & $\$$ & 0.28 & $\$$ & 0.28 \\
\hline Solar PV Electric & kW-hr & $\$$ & - & $\$$ & - & $\$$ & 0.60 & $\$$ & 0.60 \\
\hline
\end{tabular}

